\title{
Signal and Imaging Sciences Workshop 1999 Proceedings
}

\author{
J. V. Candy
}

January 5, 2001

U.S. Department of Energy

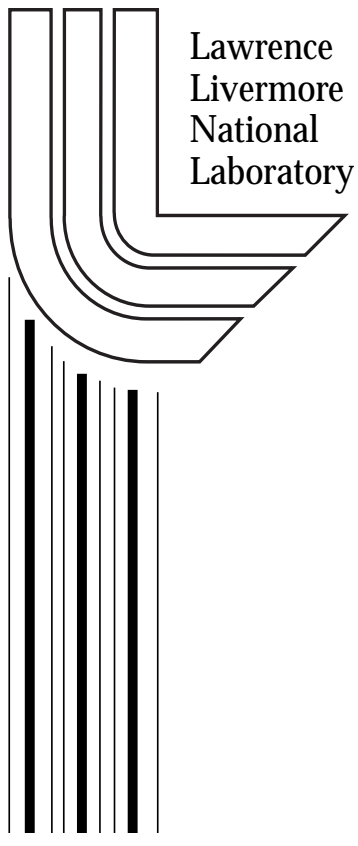




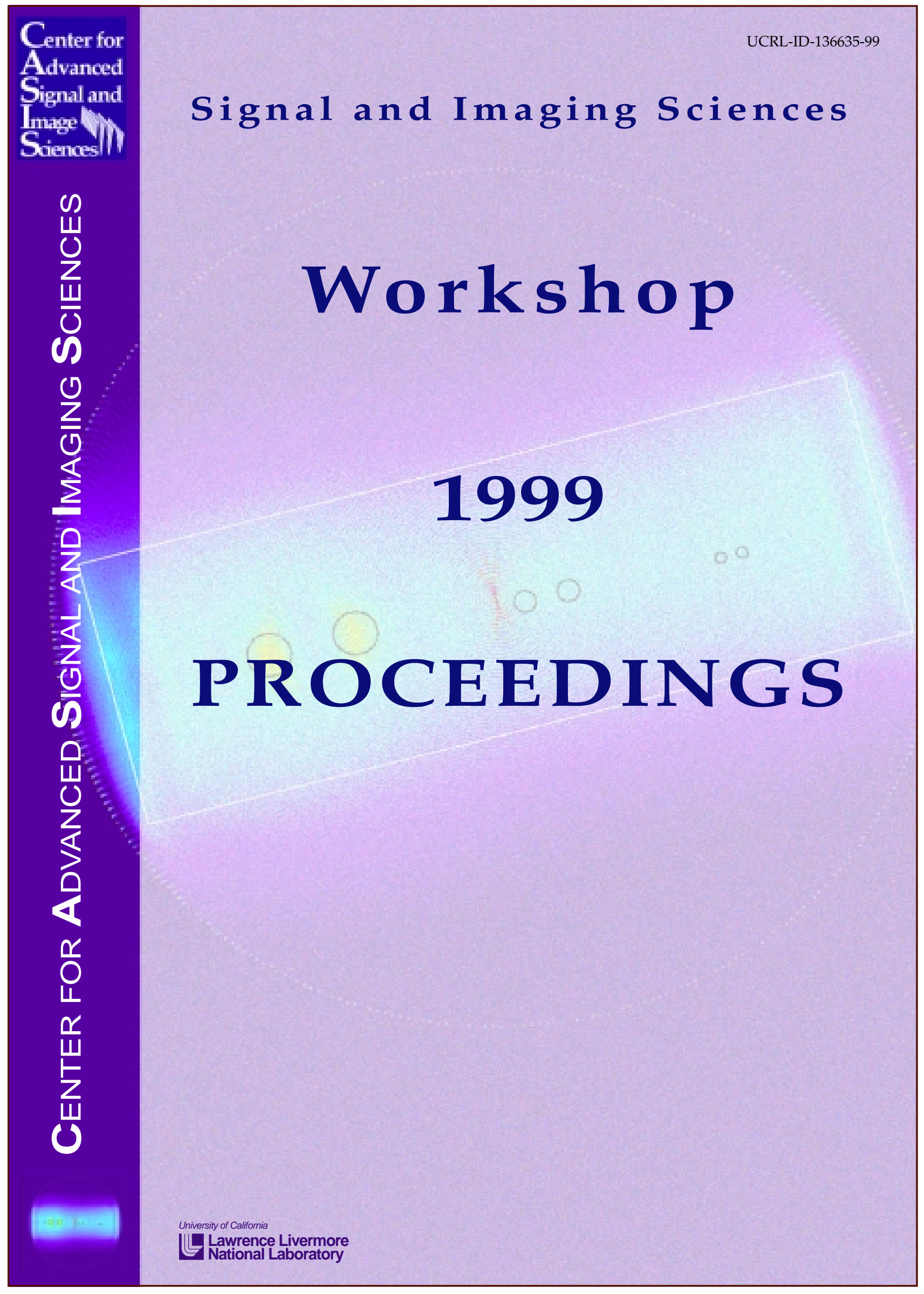




\section{DISCLAIMER}

This document was prepared as an account of work sponsored by an agency of the United States Government. Neither the United States Government nor the University of California nor any of their employees, makes any warranty, express or implied, or assumes any legal liability or responsibility for the accuracy, completeness, or usefulness of any information, apparatus, product, or process disclosed, or represents that its use would not infringe privately owned rights. Reference herein to any specific commercial product, process, or service by trade name, trademark, manufacturer, or otherwise, does not necessarily constitute or imply its endorsement, recommendation, or favoring by the United States Government or the University of California. The views and opinions of authors expressed herein do not necessarily state or reflect those of the United States Government or the University of California, and shall not be used for advertising or product endorsement purposes.

This report has been reproduced directly from the best available copy.

Available to DOE and DOE contractors from the Office of Scientific and Technical Information P.O. Box 62, Oak Ridge, TN 37831

Prices available from (615) 576-8401, FTS 626-8401

Available to the public from the National Technical Information Service

U.S. Department of Commerce 5285 Port Royal Rd.,

Springfield, VA 22161

This work was performed under the auspices of the U.S. Department of Energy by University of California Lawrence Livermore National Laboratory under contract No. W-7405-Eng-48. 


\section{Table of Contents}

\section{NOVEMBER 11 OPENING PRESENTATIONS}

A Microphone Array for Hearing Aids, Dr. Bernard Widrow - Keynote Speaker: 6

\section{SPEECH PROCESSING}

Background Speaker Noise Removal Using Combined EM

Sensor / Acoustic Signals, Larry Ng

Speaker Verification Performance Comparison Based on Traditional

and EM Sensor Pitch Extraction, Todd Gable

Defining a Human Voiced Excitation Function using Glottal

Electromagnetic Micro Power Sensors (GEMS), Greg Burnett .................................................................. 38

Synapse TAP ${ }^{\mathrm{TM}}$ Universal Access Technology, Martin Tibor ……..................................................................... 48

\section{COMPUTED TOMOGRAPHY}

Confocal Ultrasound Imaging through Acoustically Thick Media, Sean Lehman

Filtered Backprojection for Low Contrast Medical Ultrasound

Tomography Using Matlab, David Scott .......

Optimal Beam Hardening Correction for X-Ray Tomography, Jesse Kolman .

Performance Evaluation of Amorphous-Silicon Flat Panel Array

for X-Ray Digital Radiography and Computed Tomography, Ken Dolan

\section{ASTRONOMICAL IMAGING}

Speckle Imaging of Saturn's Moon Titan, Seran Gibbard..

Astronomical Imaging Fourier Transform Spectroscopy:

Technology and Techniques, Ron Wurtz...

"First Light" Science Results From the Keck Telescope

Adaptive Optics System, Claire Max. 99

Searches for Low Mass Extra-solar Planets, Jian Ge 108

Demonstration of a Diffractive Telescope, Sham Dixit

\section{IMAGE PROCESSING}

Detection of Vertical Obstructions in SAR Images, Sailes Sengupta

Use of Morphological Operators and Pattern Recognition Techniques

for Sorting the FIRST Data, Deanne Proctor.

Non-invasive Recovery of Local Properties of Deforming Objects

from Registered Range Data, Leonid Tsap

Subsurface Gap Depth Detection by Infrared Imaging

Using a Surface Heat Pulse, Charles Landram 
NOVEMBER 12 OPENING PRESENTATIONS

Overview of Engineering, Spiros Dimolitsas - Associate Director, Engineering

A Retrospective on Computer Vision Research, Dr. Avi Kak, Keynote Speaker 158

Overview of Biopatheogen Detection Technology at LLNL, Fred Milanovich 159

\section{PROCESSING FOR COMPLEX SYSTEMS}

Overview of the Center for Complex Distributed Systems, Dave McCallen 162

Using Signal Processing Techniques to Improve Finite Element Modeling, Greg Burnett ........................ 163

Damage Detection for Enhanced Evaluation of Structures, Greg Clark ................................................... 171

Parabolic Reflector for Radar Mine Detection, Steve Azevedo 181

\section{SIGNAL PROCESSING}

Broad Band Acoustic Ranging and Velocimetry, Dave Chambers 191

Grating Lobe Reduction in Large Element, 2-Dimensional Phased Arrays, Jan-Ulco Kluiwstra 200

Denoising Data using Wavelet Based Methods, Chuck Baldwin 201

Hematoma Detection Using MIR, David Scott 219

\section{IMAGING AND DETECTION}

Towards Automated Optical Evaluation of Protein Crystals, Pat Fitch 228

In-Situ Sensing of Nucleation and Crystal Growth of $\mathrm{Cd}_{1-\mathrm{x}}$ ZnxTe, William Choi 229

Phase Recovery in X-ray Diffraction, Abraham Szoke

\section{SIGNAL/IMAGE PROCESSING FOR NDE}

Nondestructive Characterization Center Overview, Harry Martz.... 249

Overview of NDE Activities at LLNL, Graham Thomas.... 255

Signal Processing for Laser Ultrasonic NDE, Robert Huber 265

Matched Field Imaging for NDE, James Candy. 273

Void Analysis from CT Imagery with Applications to Damage Evolution in AM60B Magnesium Alloy, Amy Waters 283

Ultrasonic Techniques for Materials Characterization and Process Monitoring, Peter Martin 297

Gamma-ray Imaging with a Segmented Germanium Detector, Greg Schmid 303 


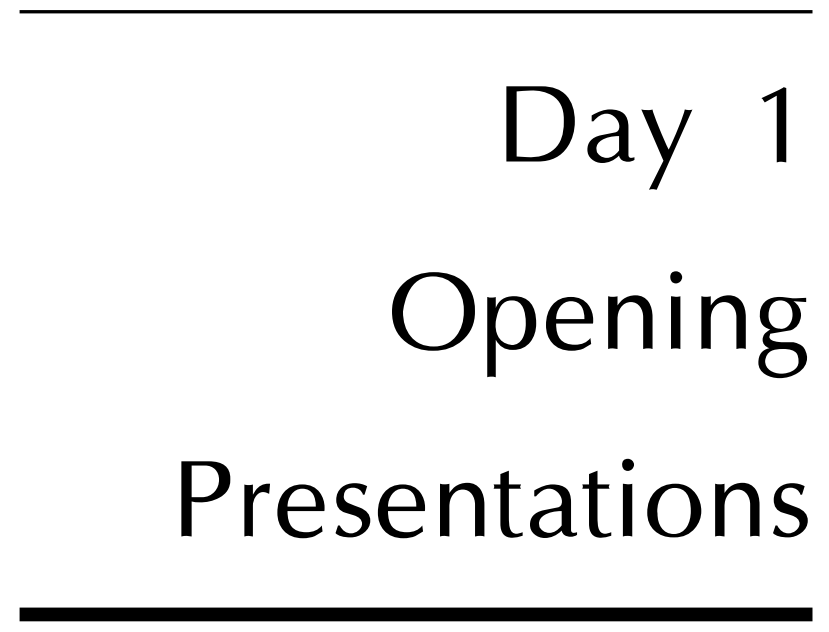




\title{
A Microphone Array for Hearing Aids
}

\author{
Bernard Widrow \\ Prof. of E.E., Stanford
}

A directional acoustic receiving system is constructed in the form of a necklace including an array of two or more microphones mounted on a housing supported on the chest of a user by a conducting loop encircling the user's neck.

Signal processing electronics contained in the same housing receives and combines the microphone signals in such a manner as to provide an amplified output signal which emphasizes sounds of interest arriving in a direction forward of the user. The amplified output signal drives the supporting conducting loop to produce a representative magnetic field. An electroacoustic transducer including a magnetic field pick-up coil for receiving the magnetic field is mounted in or on the user's ear and generates an acoustic signal representative of the sounds of interest.

The microphone output signals are weighted (scaled) and combined to achieve desired spatial directivity responses. The weighting coefficients are determined by an optimization process. By bandpass filtering the weighted microphone signals with a set of filters covering the audio frequency range and summing the filtered signals, a receiving microphone array with a small aperture size is caused to have a directivity pattern that is essentially uniform over frequency in two or three dimensions. This method enables the design of highly-directive hearing instruments, which are comfortable, inconspicuous, and convenient to use. The invention provides the user with a dramatic improvement in speech perception over existing hearing aid designs, particularly in the presence of background noise and reverberation. 


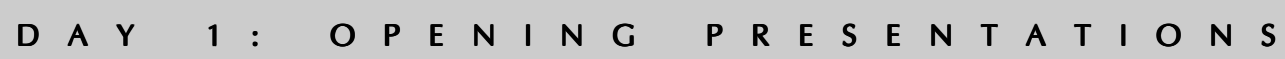
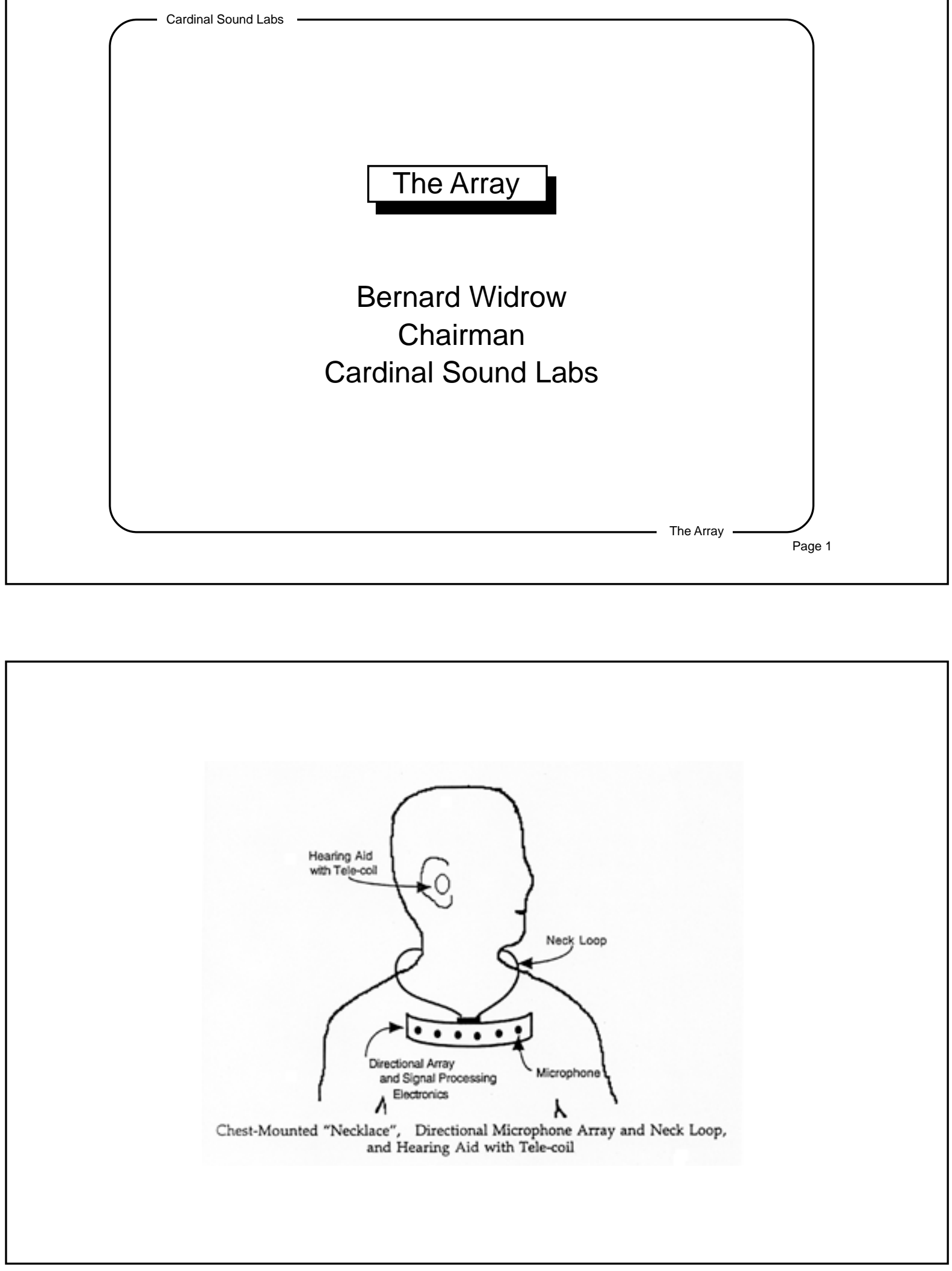


\section{A Y 1: O P E N I N G P R E S E N T A T I O N}

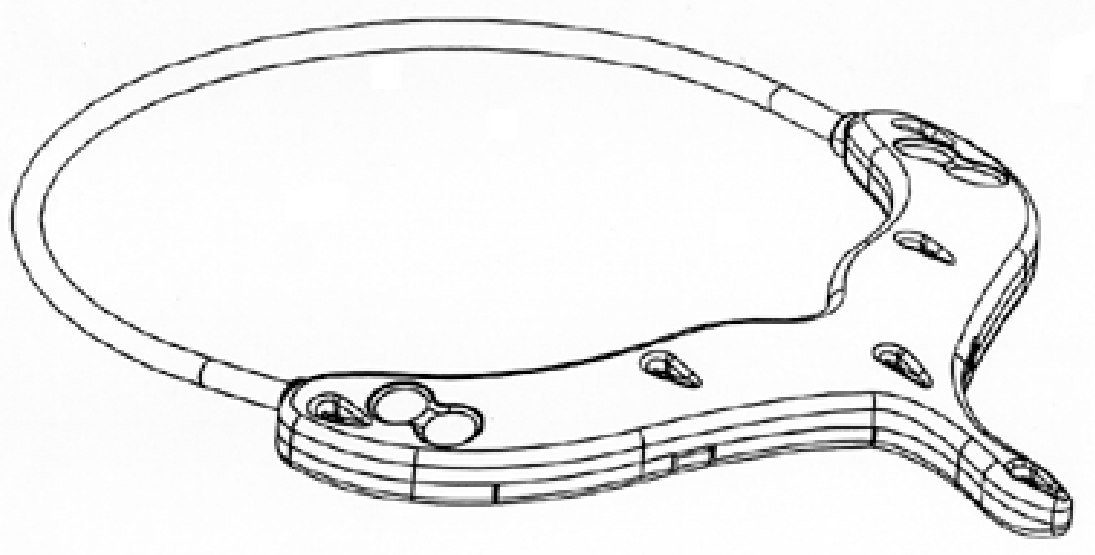

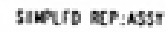

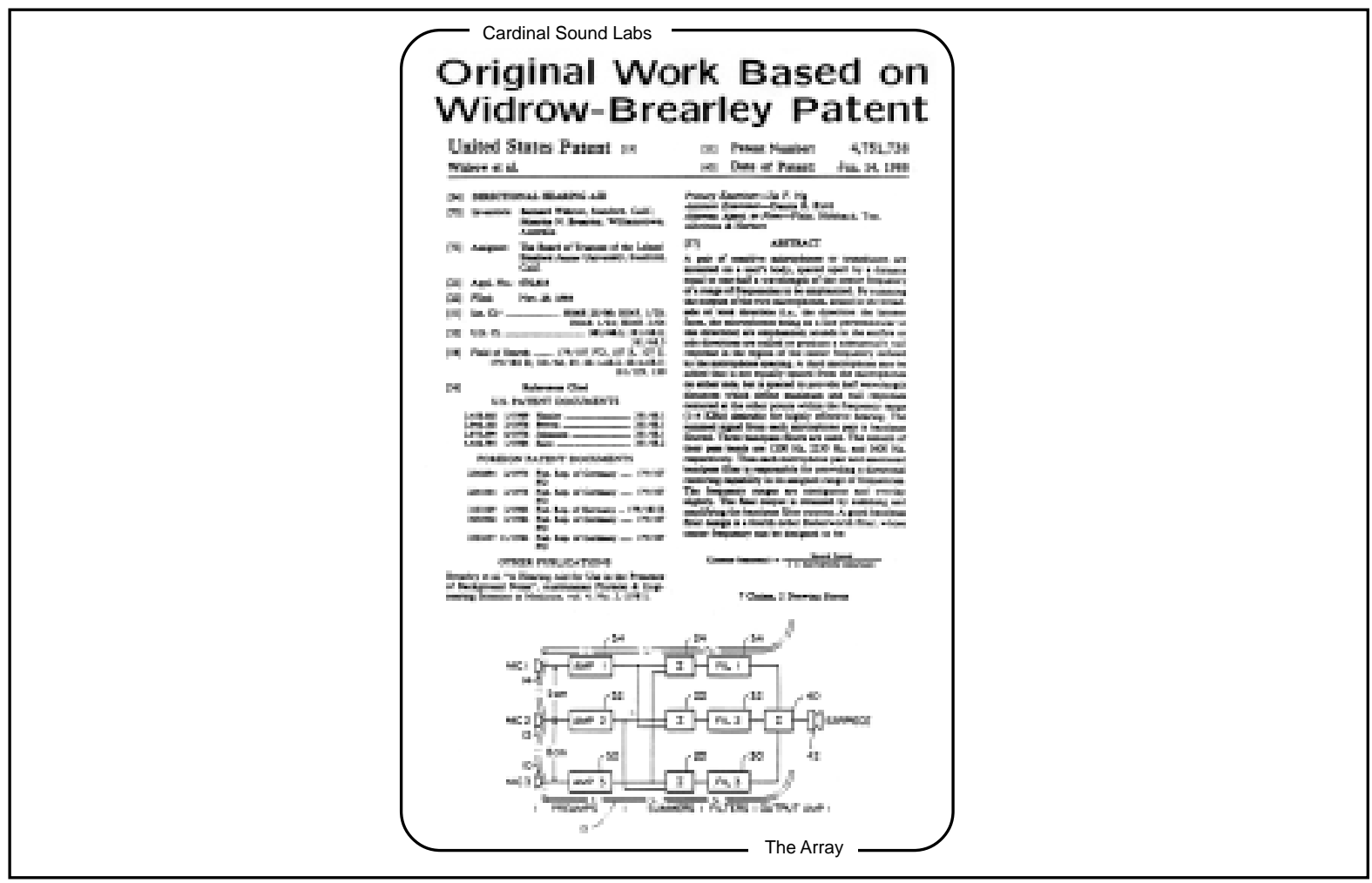


D A Y 1: O P E N I N G P R E S E N T A T I O N S

Cardinal Sound Labs

Speech Discrimination Suffers in the

Presence of Noise

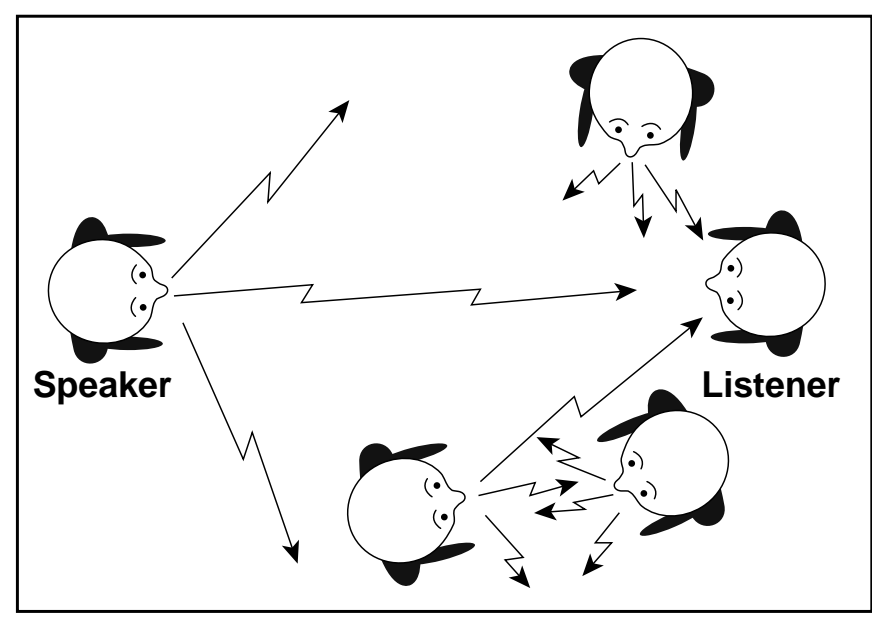

The Array

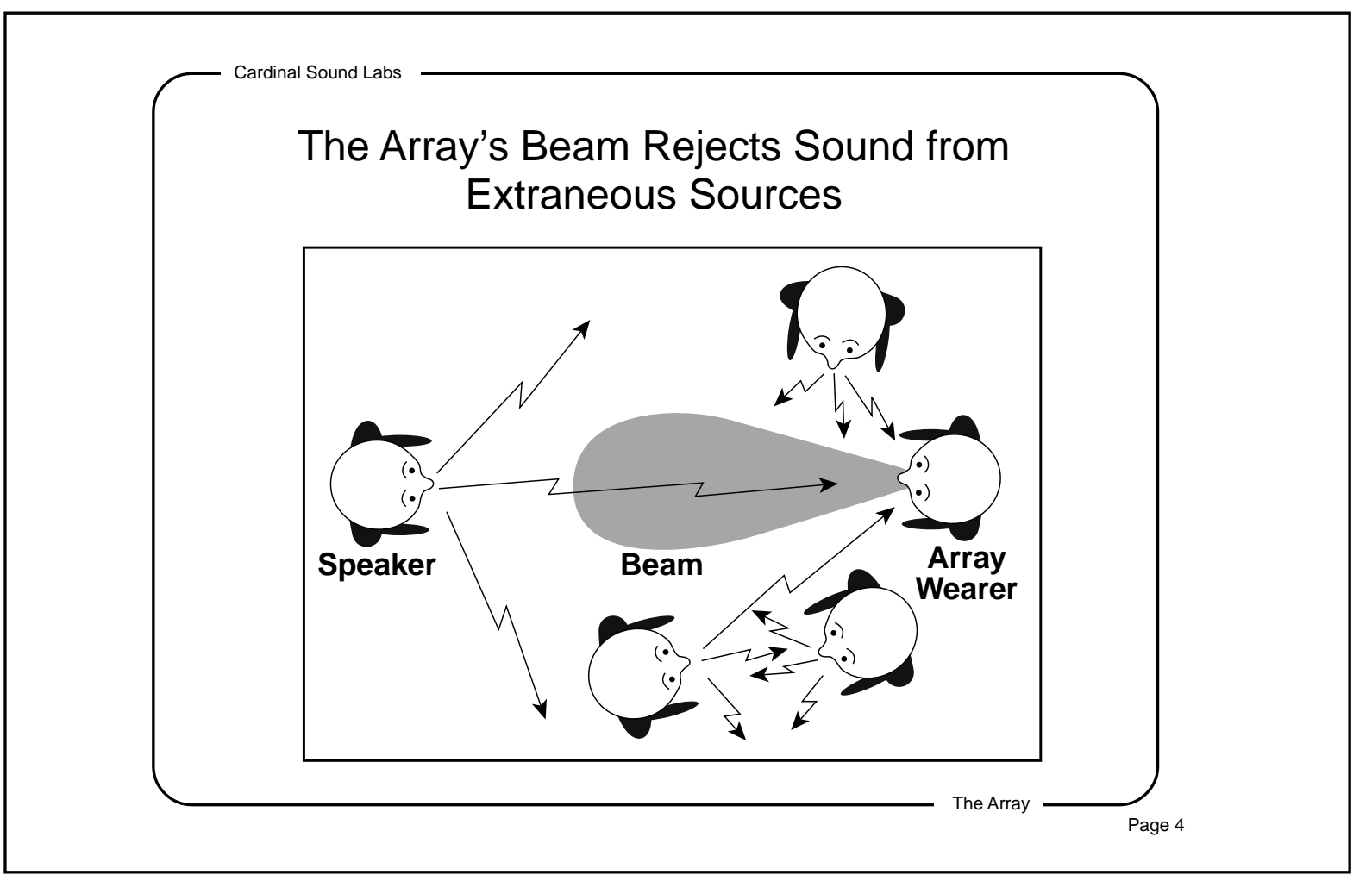


D A Y 1: O PEN I N G P R E S E N T A T I O N S
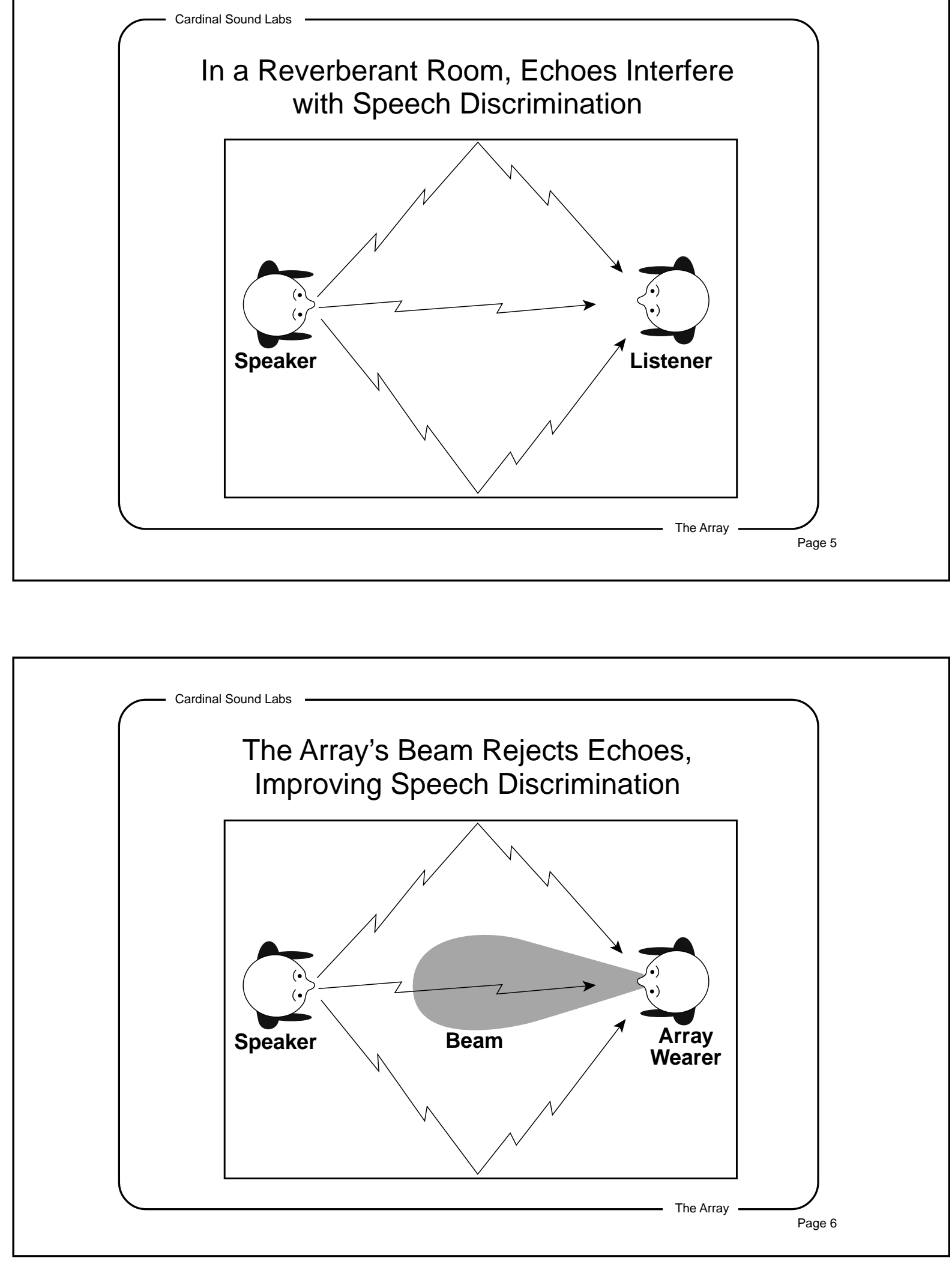
D A Y 1: O P E N I N G P R E S E N T A T I O N S

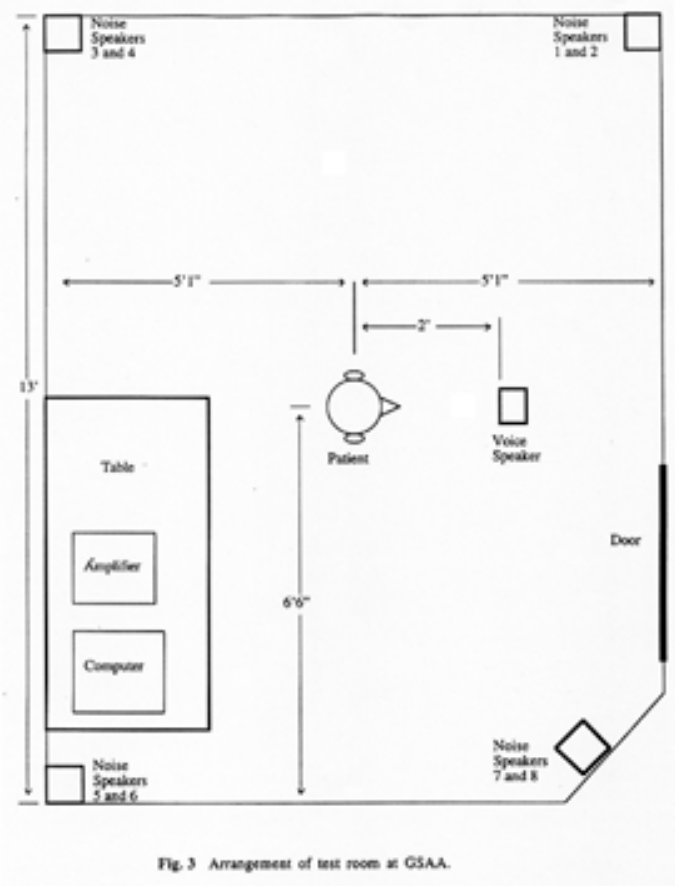

page 11
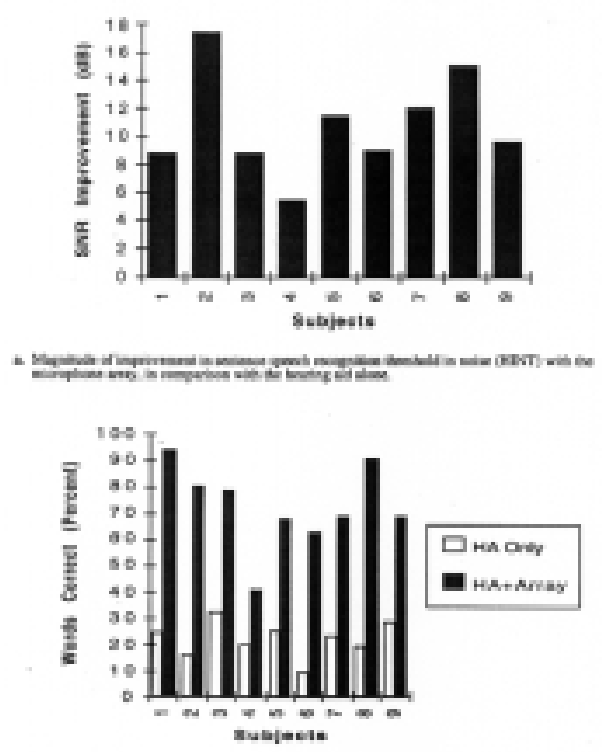

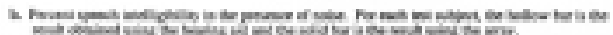

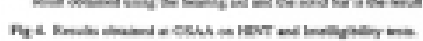

now is 

D A Y
$1:$
O P E N I N G
P R E S E N T A T I O N S

Cardinal Sound Labs

Patch Cord Enables Direct Link to TV, Sony Walkman, and other Devices

- Sony Walkman

- Television

- Telephone

- Airline Movies

- Corded Microphone for Automobile

- Infrered Hookup for Theaters

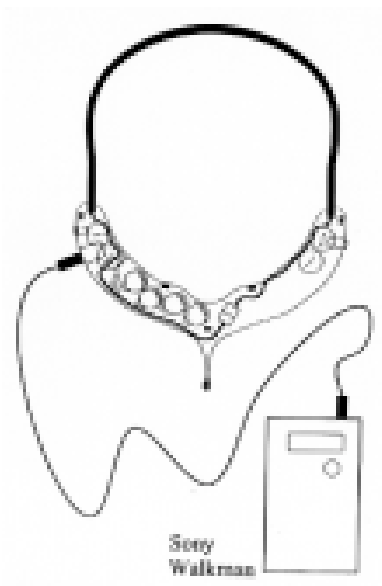

Cardinal Sound Labs

\section{The Array Has Several Other Useful Characteristics}

- Can be worn and operated under clothing or outside clothing.

- Wireless connection to hearing aid improves comfort and convenience of system.

- System greatly reduces squealing problem of hearing aids by reducing acoustic feedback.

- Battery life of final design expected to exceed one month. 

D A Y
$1:$
O P E N I N G
P R E S E N T A T I O N

Cardinal Sound Labs

\section{Companding and AGC Provides Comfort and Very Low Circuit Noise}

- Compander brings SNR of Array output down to level of Knowles microphones.

- Compander uses a 60-decibel range in $1201 / 2$ decibel steps on front and back end.

- Array has infinity-to-one AGC with user adjustable sensitivity

(kneepoint) control.

- Sensitivity adjustment allows feedback and low-level sounds to be removed without affecting nearby sounds.

- Companding, AGC, and sensitivity control also applied to external audio input.
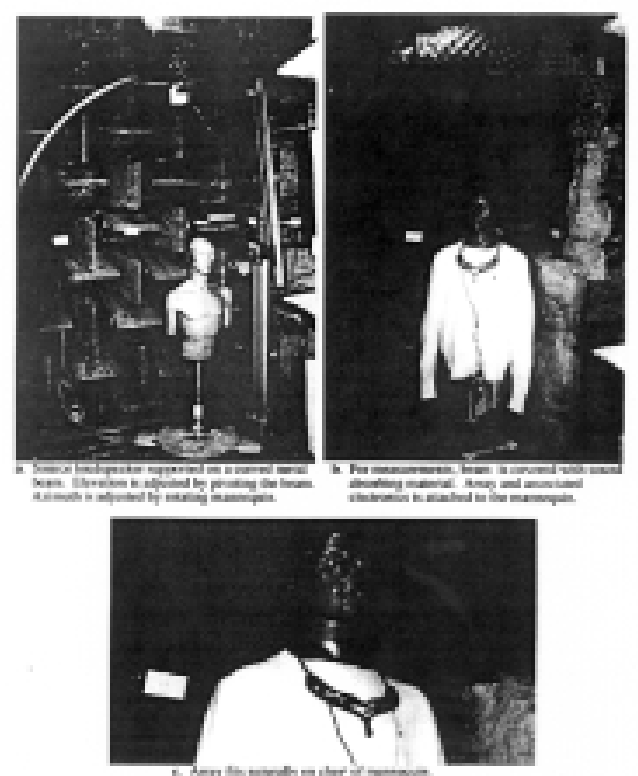

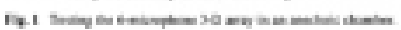



D A Y
O P E N I N G
P R E S E N T A T I O N S
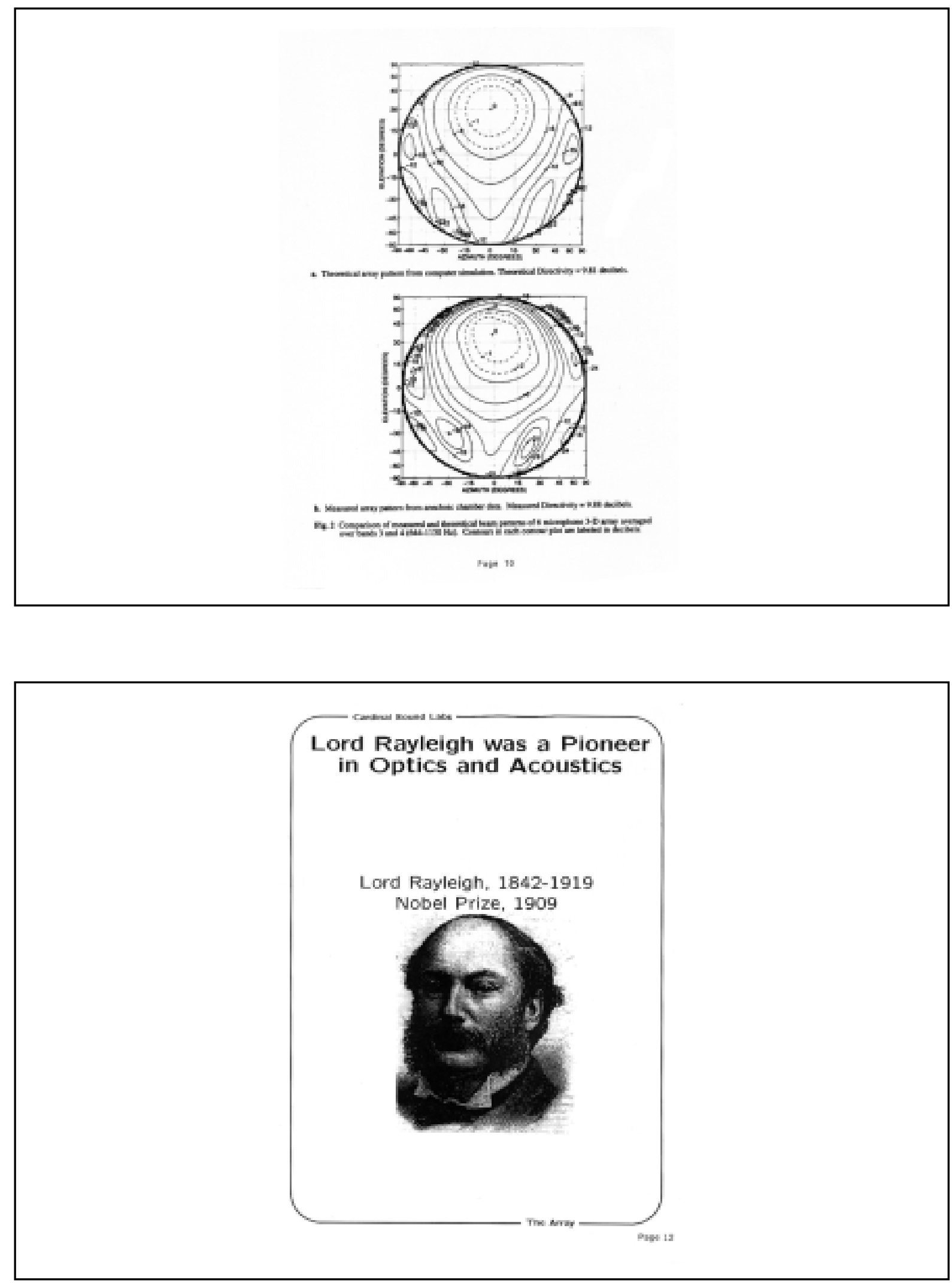

D A Y
$1:$
O P E N I N G
P R E S E N T A T I O N

Cardinal Sound Labs

\section{Lord Rayleigh's Formula Predicts}

Minimum Size for an Array

Lord Rayleign's formula is:

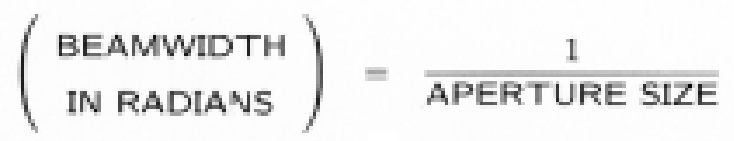

This formula tells us that to get a beamwidth of $60^{\circ}$

(approximately 1 radian) at frequencies as low as $\mathbf{5 0 0}$ Hertz, the width and height of the Cardinal Sound Labs' Array would need to be approximately two feet.

Cardinal Sound Labs

\section{New Prototypes Get Past Size}

Limitation

We have overcome this limitation without violating the laws of physics, and this is the substance of our most recent patent

application. The Array is approximately 7.7 inches wide and 5 inches high. 
D A Y 1: O P E N I N G P R E S E N T A T I O N S
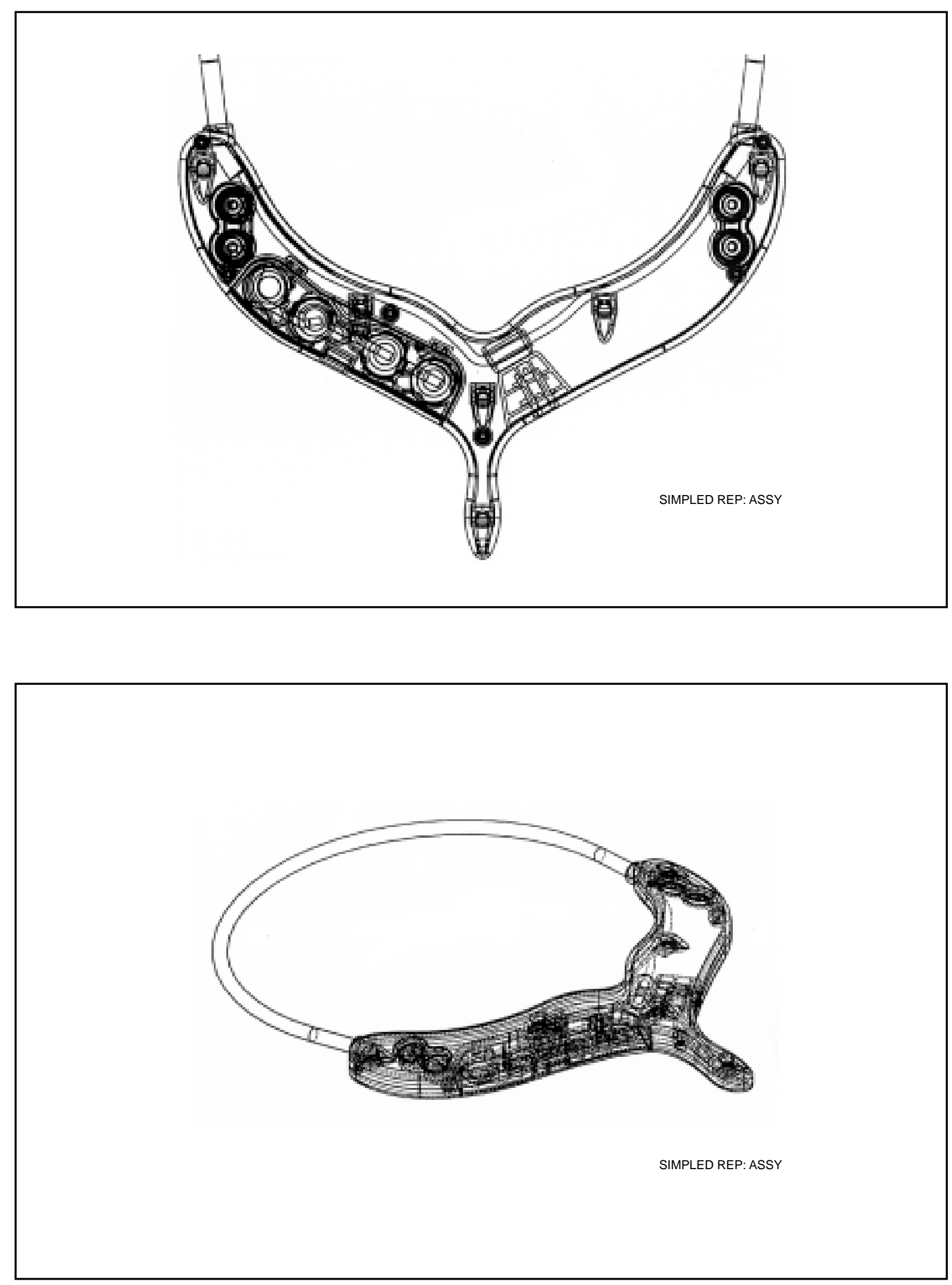
D A Y 1: O P E N I N G P R E S E N T A T I O N S

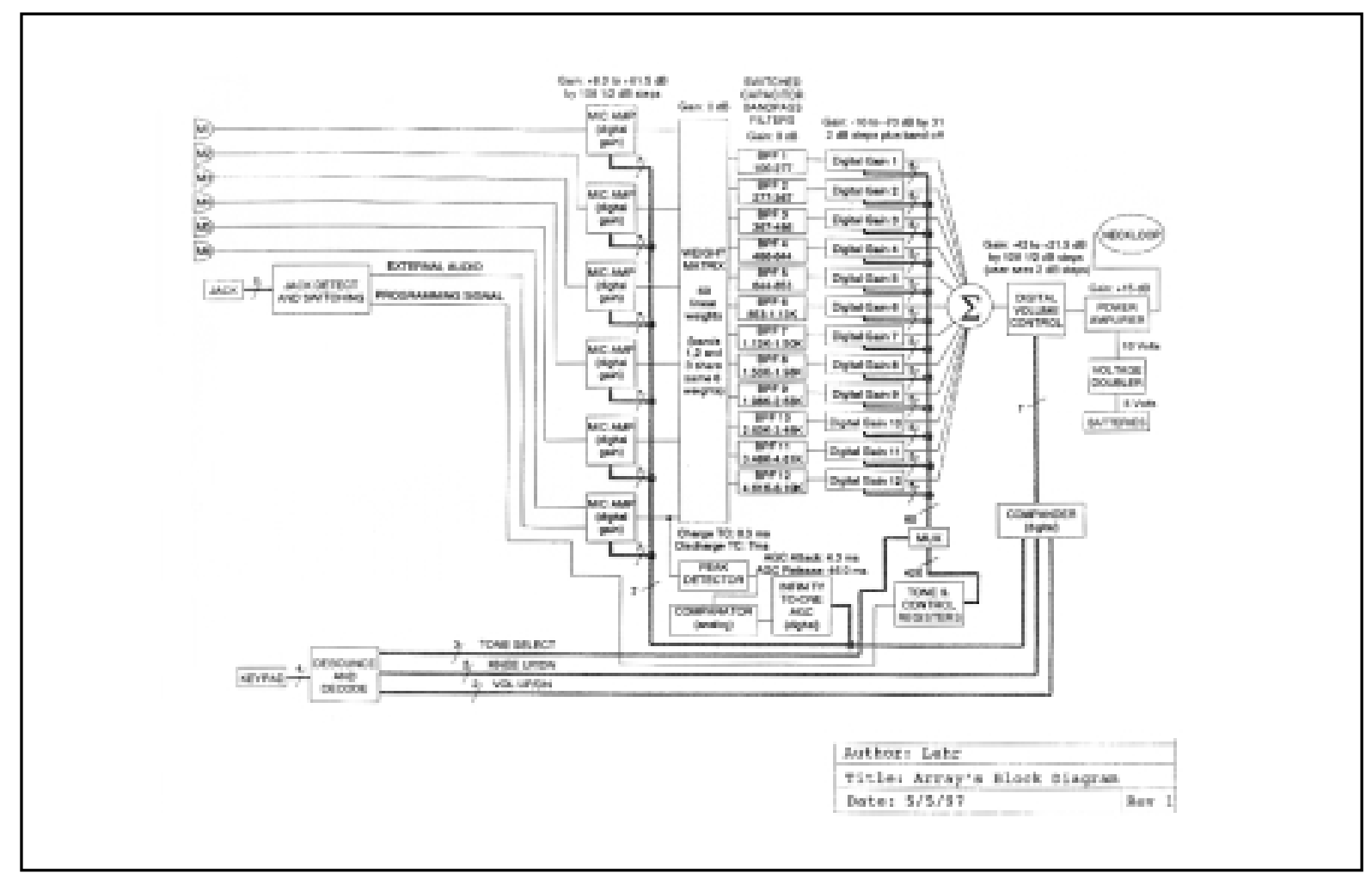




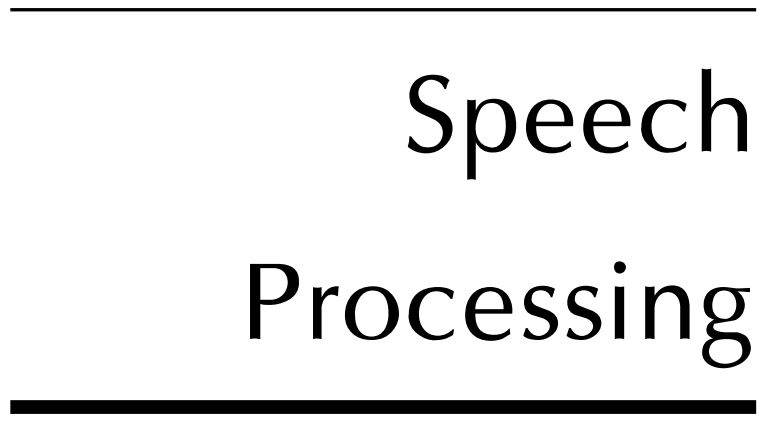




\title{
Background Speaker Noise Removal Using Combined EM Sensor/Acoustic Signals
}

\author{
L.C. Ng, G. C. Burnett, J. F. Holzrichter, and T. J. Gable
}

Recently, very low power EM radar-like sensors have been used to measure the macroand micro-motions of human speech articulators as human speech is produced [ see Holzrichter et. al. JASA. 103 (1) 622, (1998)]. These sensors can measure tracheal wall motions, associated with the air pressure build up and fall as the vocal folds open and close, leading to a voiced speech excitation function. In addition, they provide generalized motion measurements of vocal tract articulator gestures that lead to speech formation. For example, tongue, jaw, lips, velum, and pharynx motions have been measured as speech is produced. Since the EM sensor information is independent of acoustic air pressure waves, it is independent of the state of the acoustic background noise spectrum surrounding the speaker. By correlating the two streams of information together, from a microphone and (one or more) EM sensor signals, to characterize a speaker's speech signal, much of the background speaker noise can be eliminated in real-time. This paper presents the improved performance of glottal EM sensors (GEMS) based algorithms in suppressing a variety of background speaker noise. [Work supported by NSF and DOE.]

\section{Background Speaker Noise Removal Using Combined EM Sensor/Acoustic Signals}

\author{
Signal and Imaging Sciences Workshop
}

Nov 11, 1999

Presented by:

\section{Lawrence C. Ng, Ph.D.}

Group Leader

Signal/Image Processing and Control Group

Defense Sciences Engineering Division

Collaborators:

Dr. J. Holzrichter, Dr. G. Burnette, Mr. T. Gable

Lawrence Livermore National Laboratory

P.O. Box 808, L-491, Livermore, CA, 94551 
- Brief Description of GEMS (sensor) Characteristics

- Application to Speech Processing

- The Background Noise Removal Problem

- The Wiener Filter Solution

- The Glottal Windowing (GWIN) Filter Solution

- The Glottal Correlation (GCOR) Filter Solution

- Summary

Micro Power EM Sensors (Radar-related) Were Developed as High Speed Diagnostic Tools for a Laser Fusion Experiment at LLNL

- Emits pulses of very low power $(<1 \mathrm{nW})$ and short duration

- Center frequency is around $2.5 \mathrm{GHz}$ (but adjustable)

- Due to short duration of pulse it is composed of many different frequencies

- Emits pulses at a $2 \mathrm{MHz}$ PRF which results in high signal to noise ratio (many returns can be averaged)

- Operating in a field disturbance mode, it is an excellent motion and vibration detector
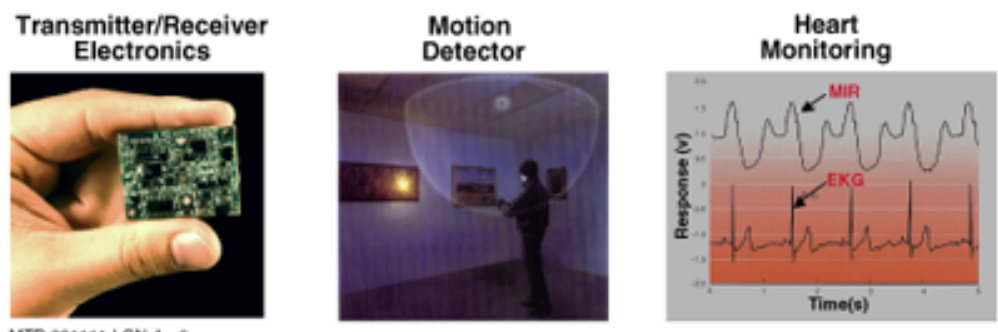

MTP-991111-LCN-A-3 


\section{EM Sensors Derive the Signal by Measuring The Differential Positions of the Returned Wave}

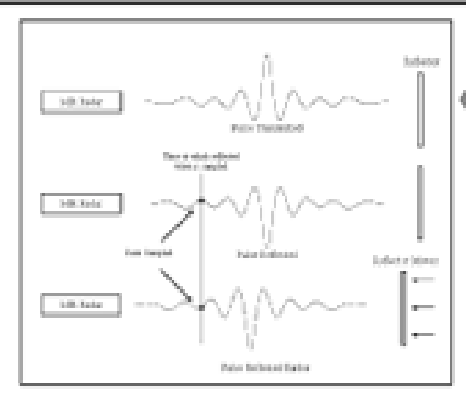

EM sensor measures the changes in signal amplitude resulting from the movement of an object

Good correlation of EM sensor signal to high speed video recording of vocal folds motion was obtained
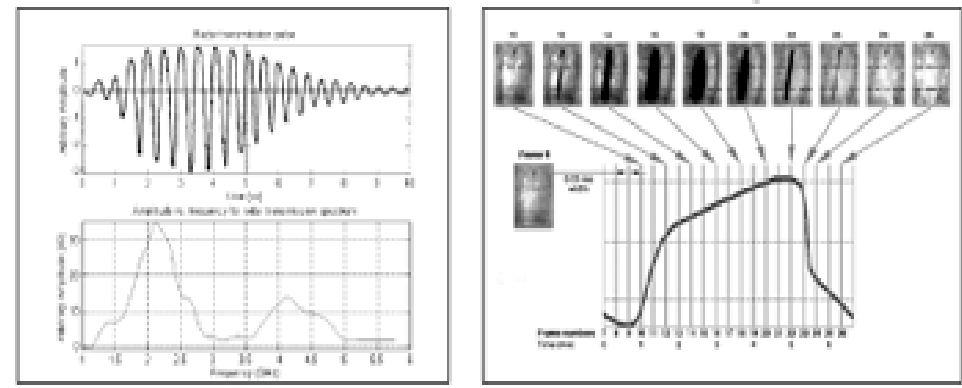

MTP.PM111:LCN.A.A

Glottal EM Sensor (GEMS) Tissue Measurements Show Strong Correlation with Vocal-Fold Electroglottalgraphy (EGG) Signal
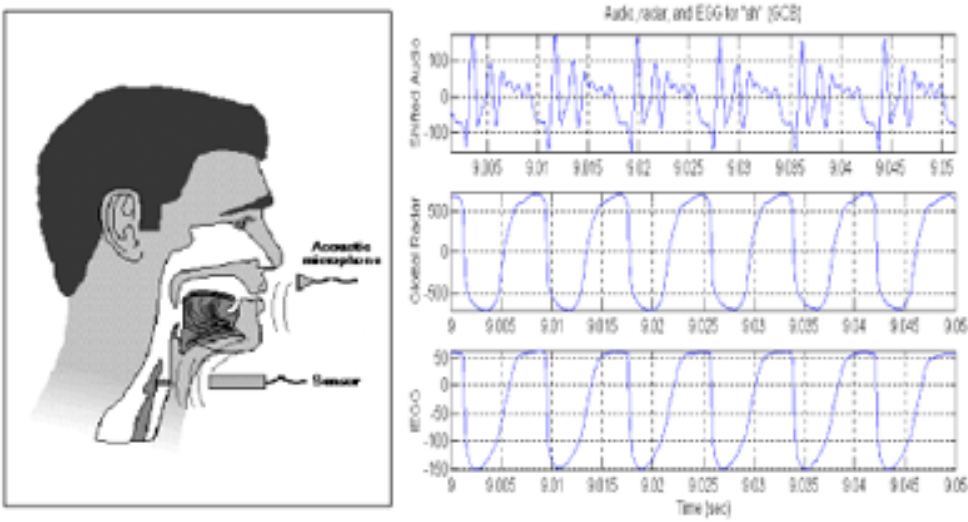

MTP-991111-LCN-A-5 


\section{Background Noise Removal Problem Must Address}

Two Major Issues: Voice detection and Noise Rejection

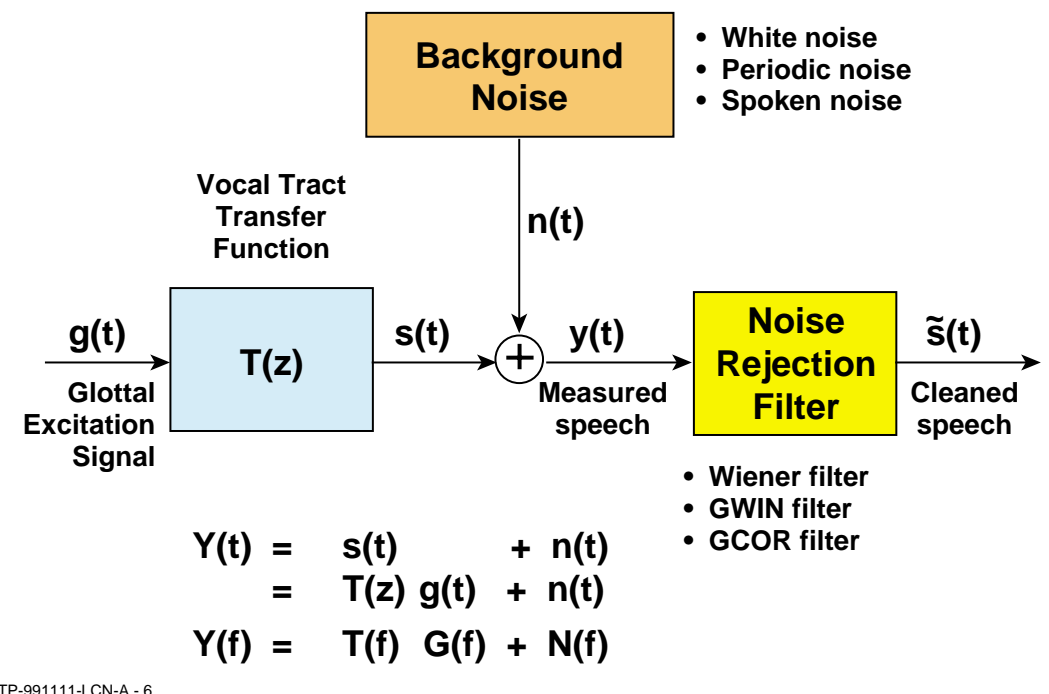

The Wiener Filter Can Optimally Minimize the Noise When the Signal and Noise Spectrum Are Available

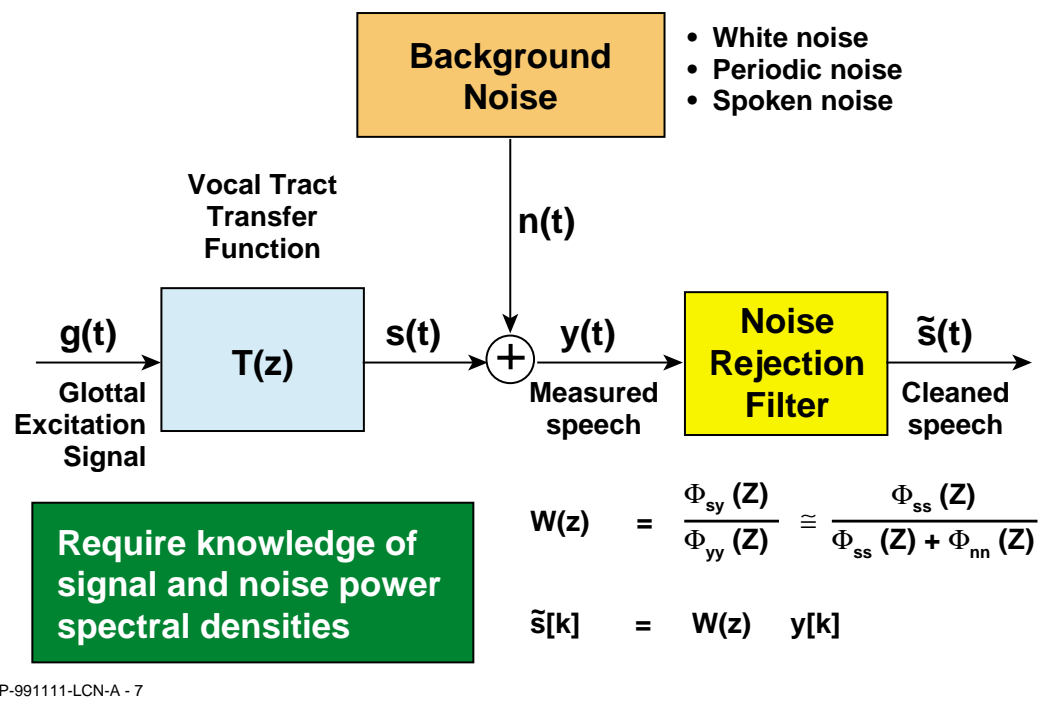




\section{The Glottal Windowing (GWIN) Filter Can Be Simply} Constructed from the Glottal EM Sensor Signal

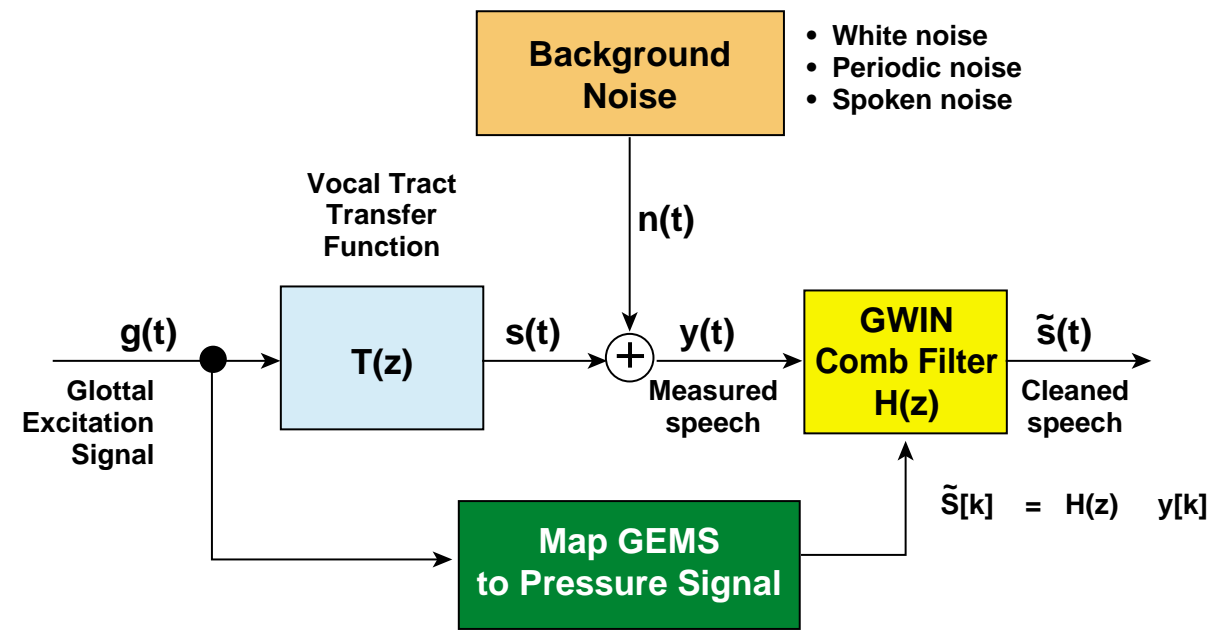

\section{Glottal Correlation (GCOR) Filter Can Be Used} To Optimally Remove the Background Noise

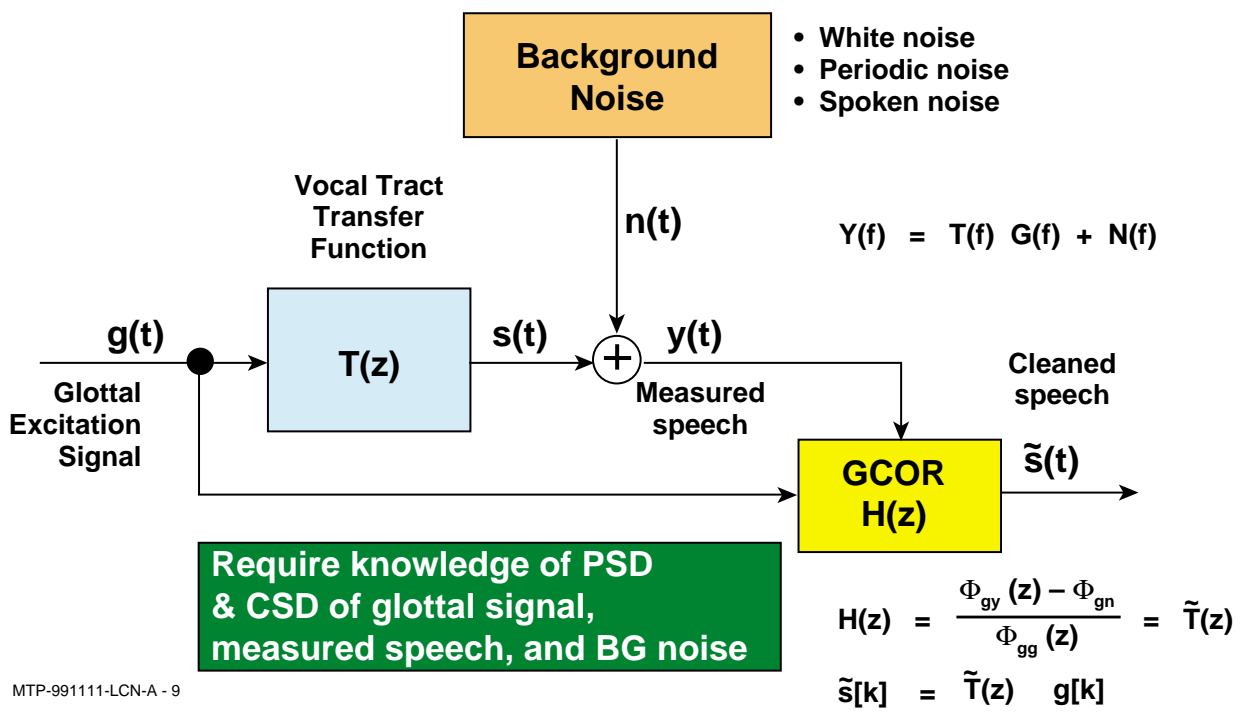




\section{GWIN Comb Filter Can Be Easily Constructed} From Glottal EM Sensor Measurements
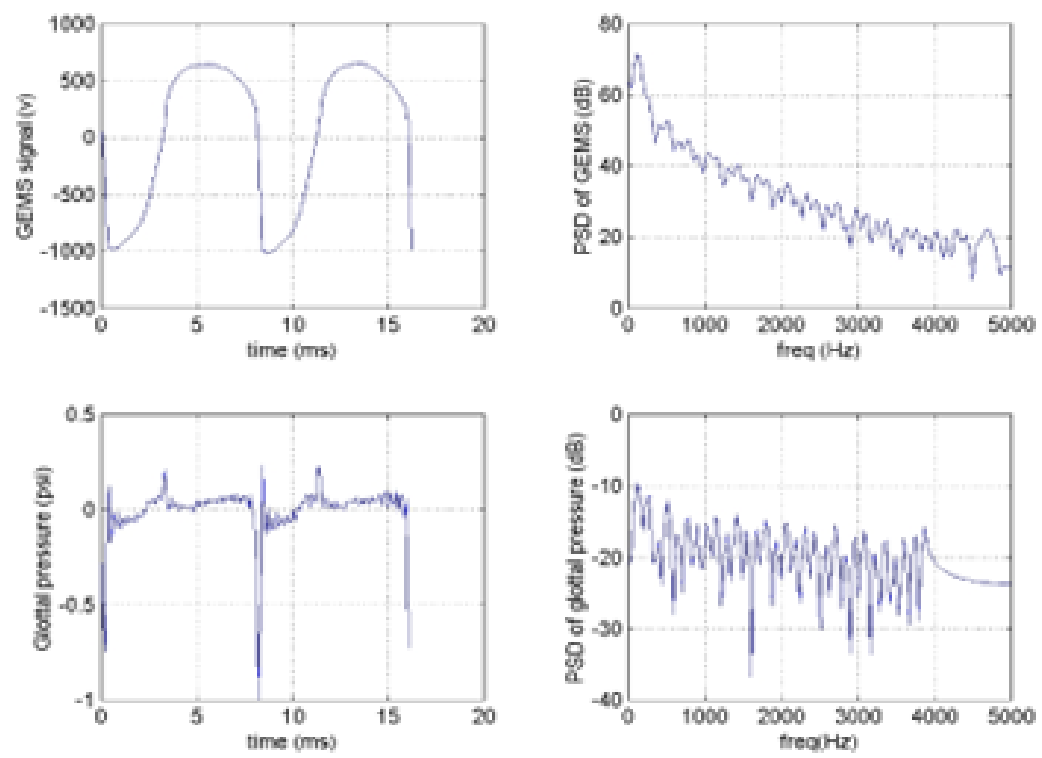

MTP-991111-LCN.A - 10

We Added the Utterance /eh/Spoken by Two Males Simultaneously as a Background Noise Test Problem
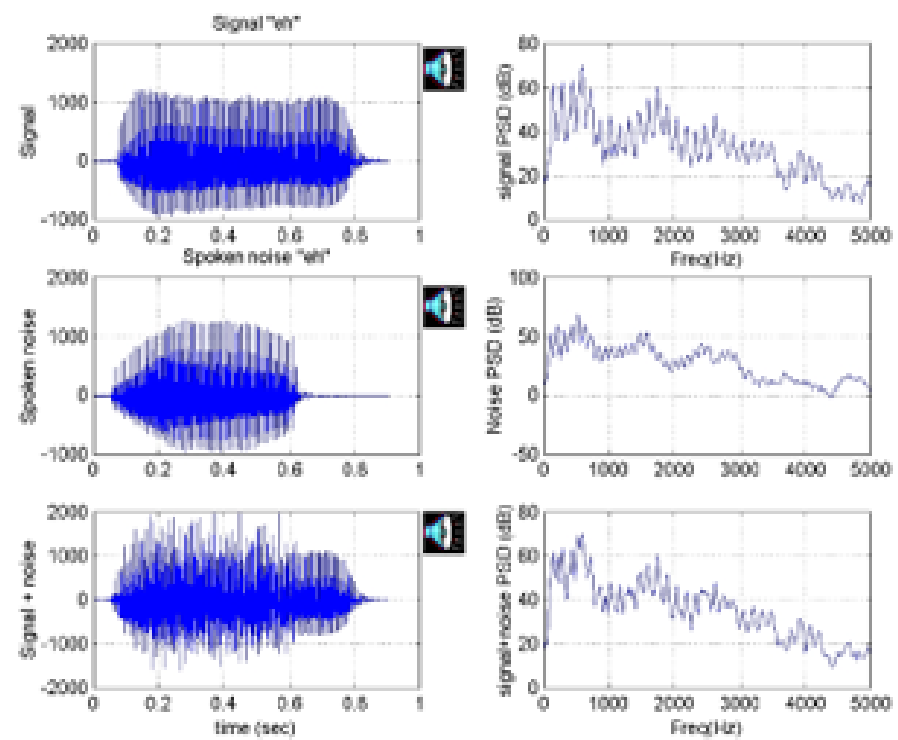
We Observed That Both GWIN and GCOR (Glottal based) Performed Better Than the Wiener Filter
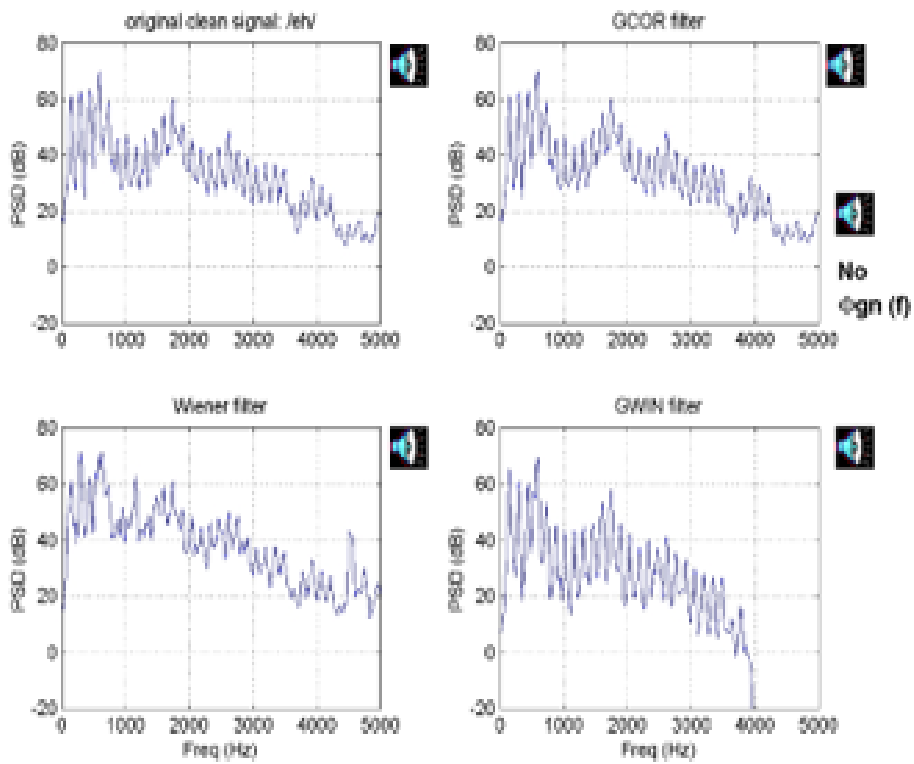

\section{Detection of Voiced and Unvoiced Boundary is Difficult in a High Background Noise Environment}
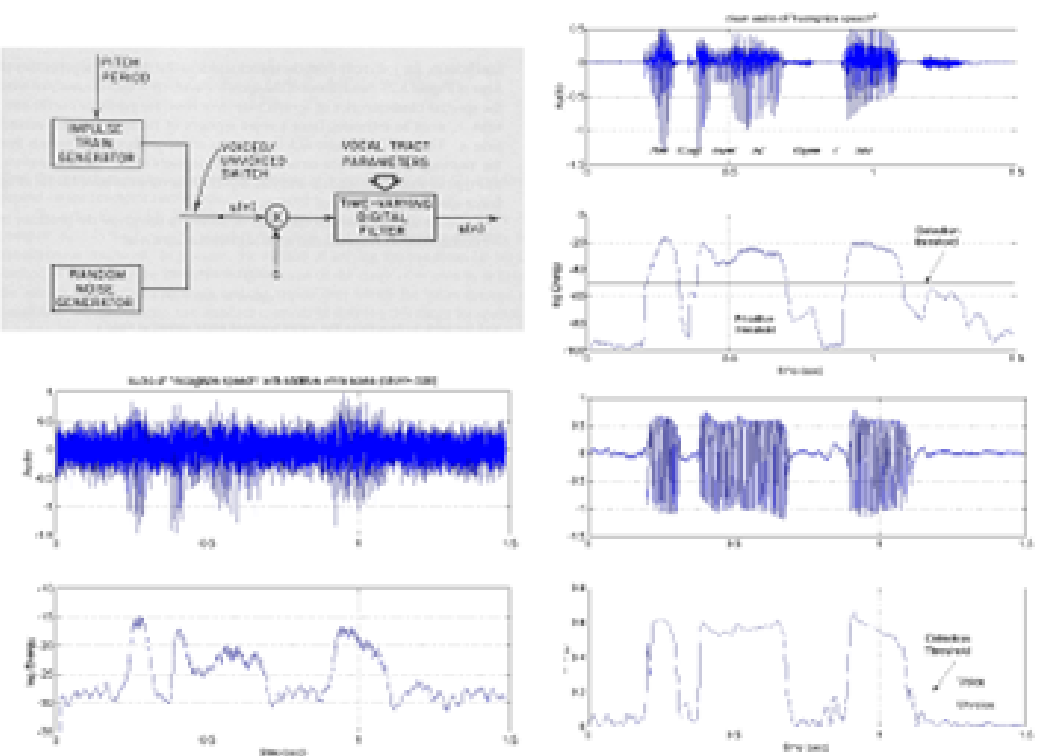

MTP.991111-LCN-A = 13 
Background White Noise Suppression with the Spoken Words: "Recognize Speech"
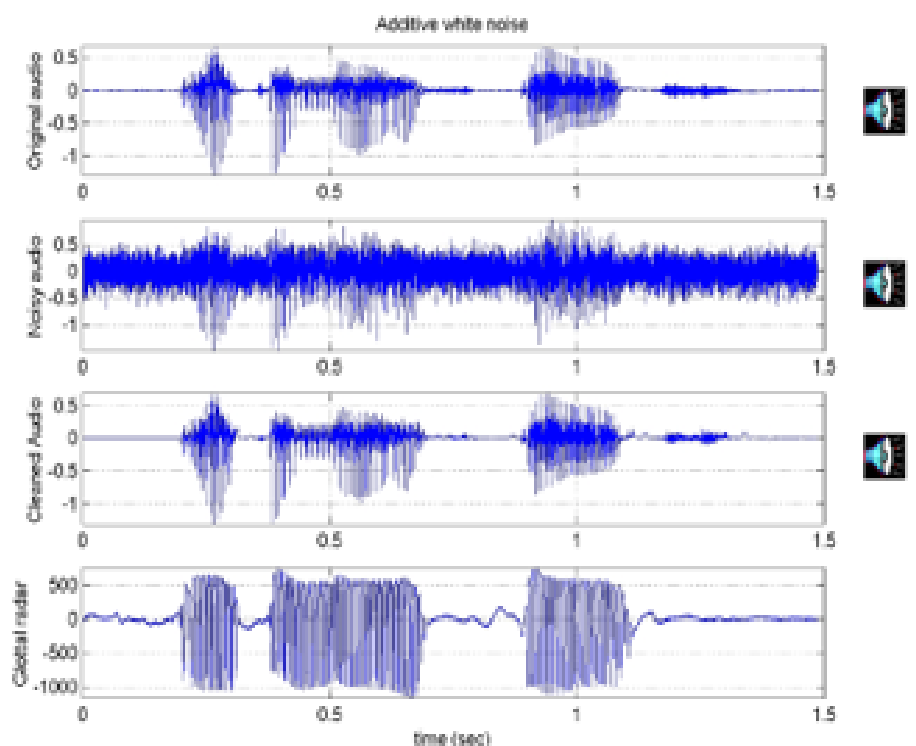

MTP.P1111-LCN-A - 14

\section{Background Periodic Noise Suppression with} the Spoken Words: "Recognize Speech"
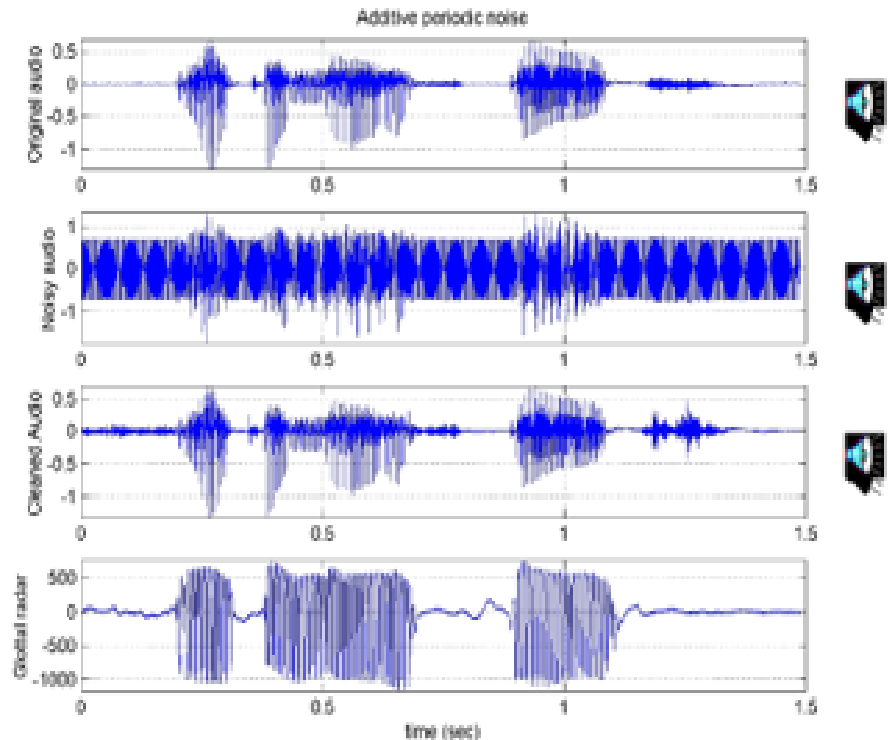

MTP-P9111-LCN-A - 15 


\section{Background Spoken Noise Suppression with} the Spoken Words: "Recognize Speech"
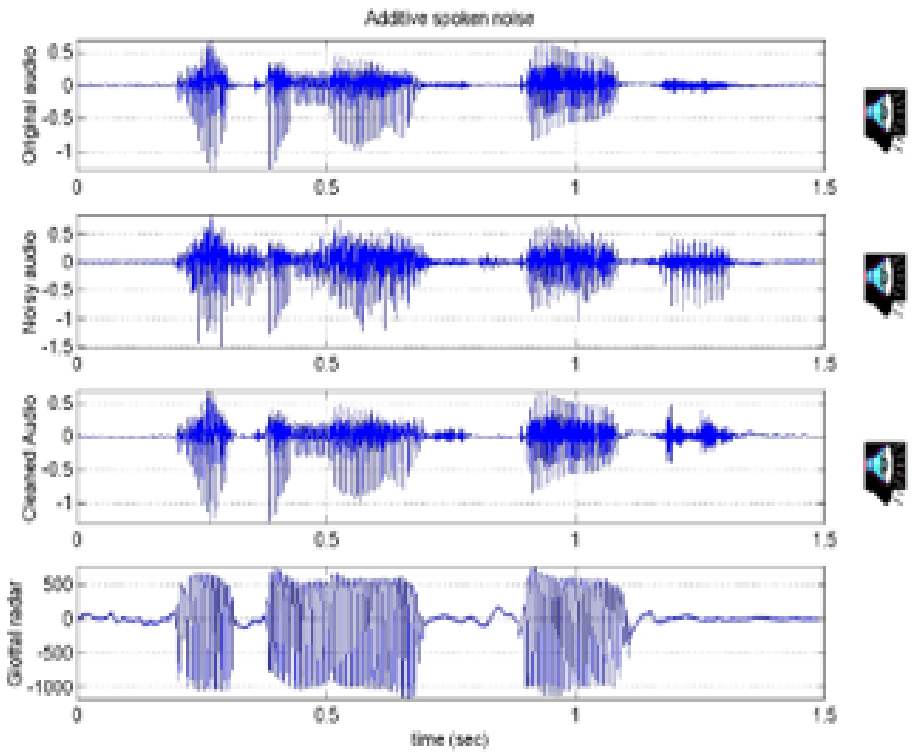

MTP-991111-LCN-A -16

GEMS-Microphone - Could Provide a Reliable and

Robust Voice Interface in a Noisy Environment
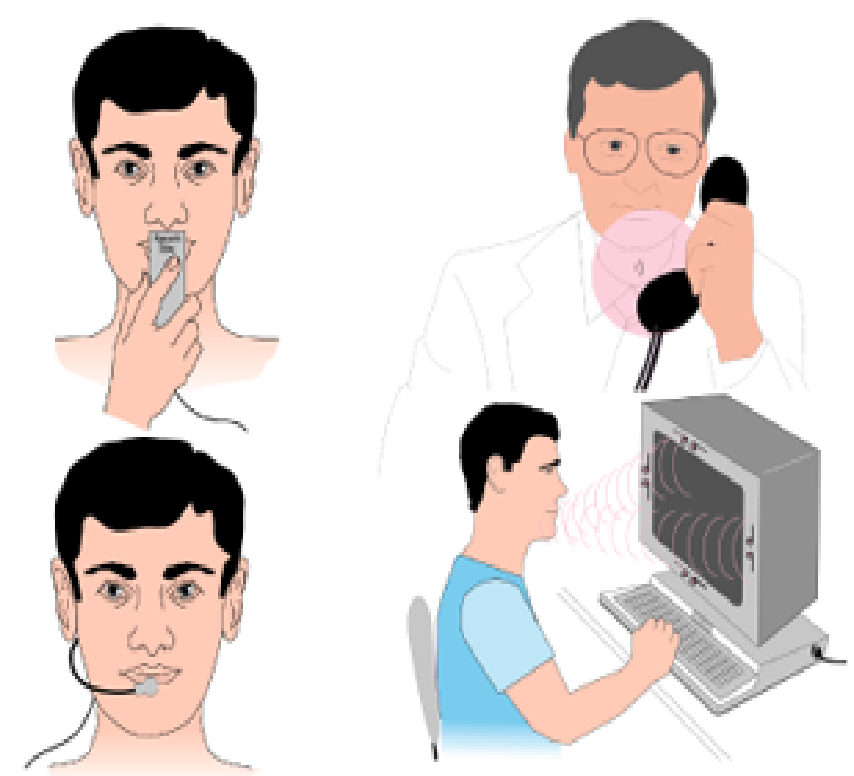

MTP-P91111-LCN:A -17 


\section{Summary and Conclusions}

- We have examined several options to remove background noise

- Wiener Filter (classical approach)

- GWIN Filter (Spectral comb filter generated from pressure waveform derived from GEMS measurement)

- GCOR Filter (make use of glottal and voice signal correlation)

- We tested the filters with three kinds of background noise

- White noise

- Periodic noise

- Spoken noise

- Both GWIN and GCOR out performed the classical Wiener filter

- Signal and noise spectrum are difficulty to obtain for transient speech signal

- GEMS is useful in detecting voiced/unvoiced speech boundaries

- Measured GEMS glottal signal is a key to remove background noise

- We applied GEMS based filters to various background noise and found that GEMS is also effective against spoken noise

- GEMS-microphone has a potential to operate in a very noisy environment 


\title{
Speaker Verification Performance Comparison Based on Traditional and Electromagnetic Sensor Pitch Extraction
}

\author{
T.J. Gable, L.C. Ng, G.C. Burnett, and J.F. Holzrichter \\ Lawrence Livermore National Laboratory
}

This work compares the speaker verification performance between a traditional acoustic-only pitch extraction to a new electromagnetic (EM) sensor based pitch approach. The pitch estimation approach was developed at the Lawrence Livermore National Laboratory (LLNL) utilizing Glottal Electromagnetic Micropower Sensors (GEMS, also see http: / / speech.llnl.gov/). This work expands previous pitch detection work by Burnett et. al. (to be published IEEE Trans. on Speech and Audio Processing) to the specific application of speaker verification using dynamic time warping. Clearly, a distinct advantage of GEMS is its insensitivity to acoustic ambient noise. This work demonstrates the clear advantage of the GEMS pitch extraction to improve speaker verification error rates. Cases with added white noise and other speech noise were also examined to show the strengths of the GEMS sensor in these conditions. The EM sensor speaker verification process operated without change over signal-to-noise (SNR) conditions ranging from $-20 \mathrm{~dB}$ to $-2.5 \mathrm{~dB}$, the acoustic algorithms became unusable at SNR exceeding $-10 \mathrm{db}$. [Work supported by NSF and DOE.]

\section{Speaker Verification Performance Comparison Based on Traditional and Electromagnetic Sensor Pitch Extraction}

CASIS November 1999

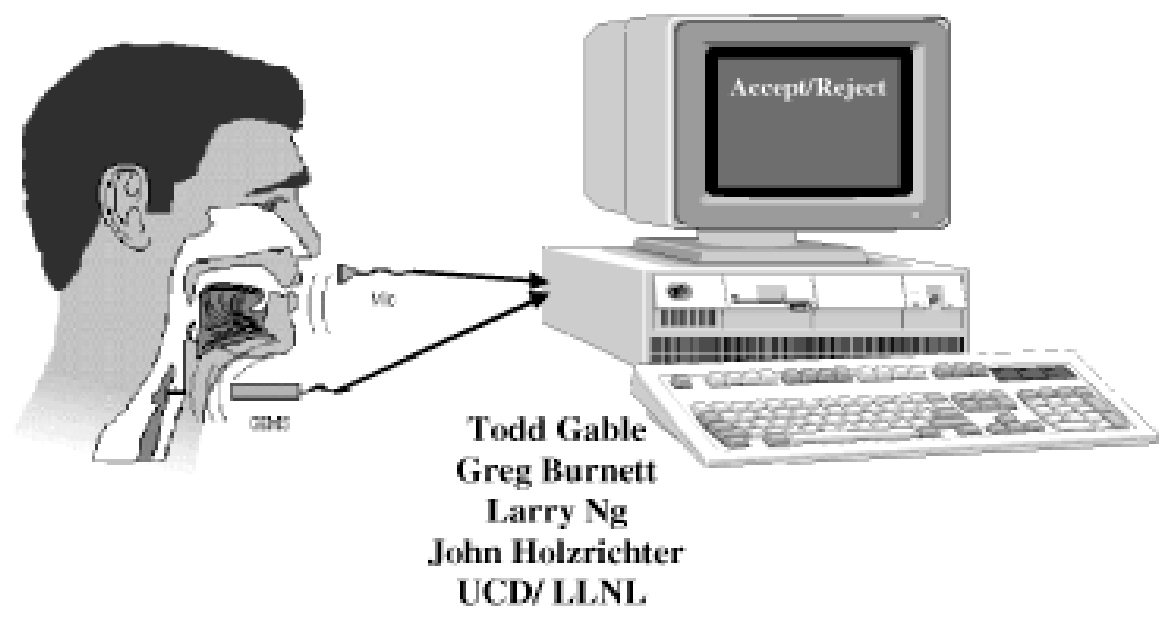


Purpose of this study is to evaluate the performance of EM sensor (GEMS) using pitch in speaker verification

- Comparing performance differences between traditional acoustic and Glottal Electromagnetic Sensor (GEMS) pitch extraction using a corpus of TIMIT sentences from 15 male speakers

- Using GEMS extracted pitch and conventional dynamic time warping verification methods lead to lower speaker verification errors

- Demonstrate the GEM sensor's insensitivity to acoustic noise

Glottal EM Sensor (GEMS) signal is well suited for voiced speech pitch extraction
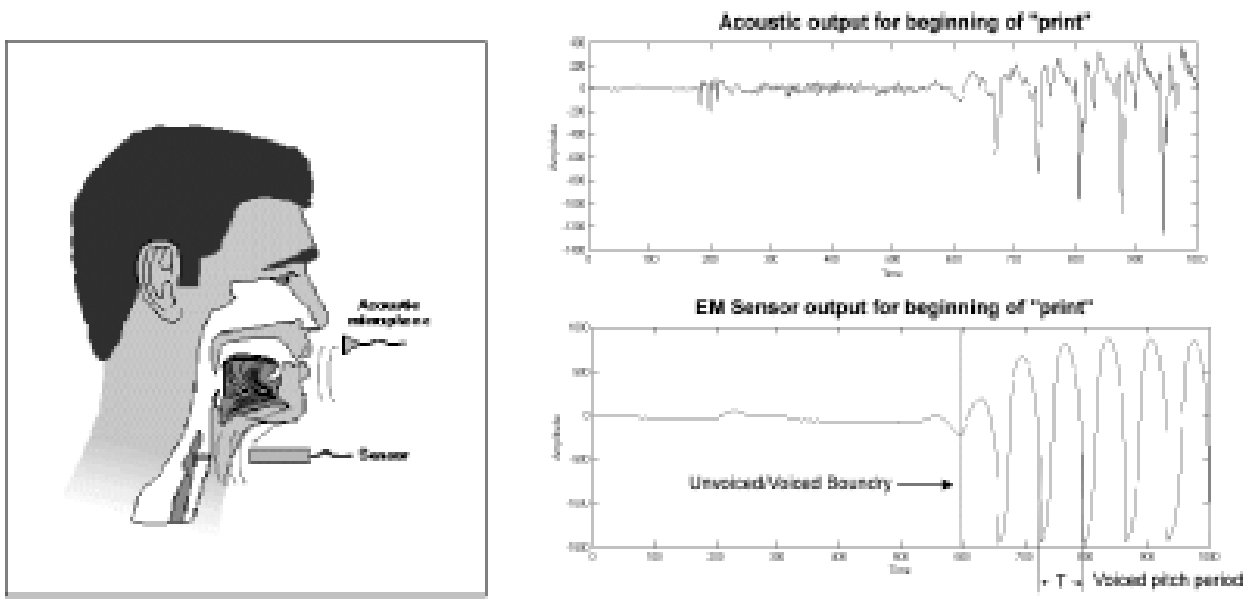
- PC Laptop with Labview and NiDaQ was used to take data

- Data was sampled at $40 \mathrm{kHz}$ and filtered and decimated to $10 \mathrm{kHz}$ in post-processing

- 15 male volunteers were used

- 12 sentences were recorded with 2 sets of 5 repetitions were recorded, separated by at least 2 weeks
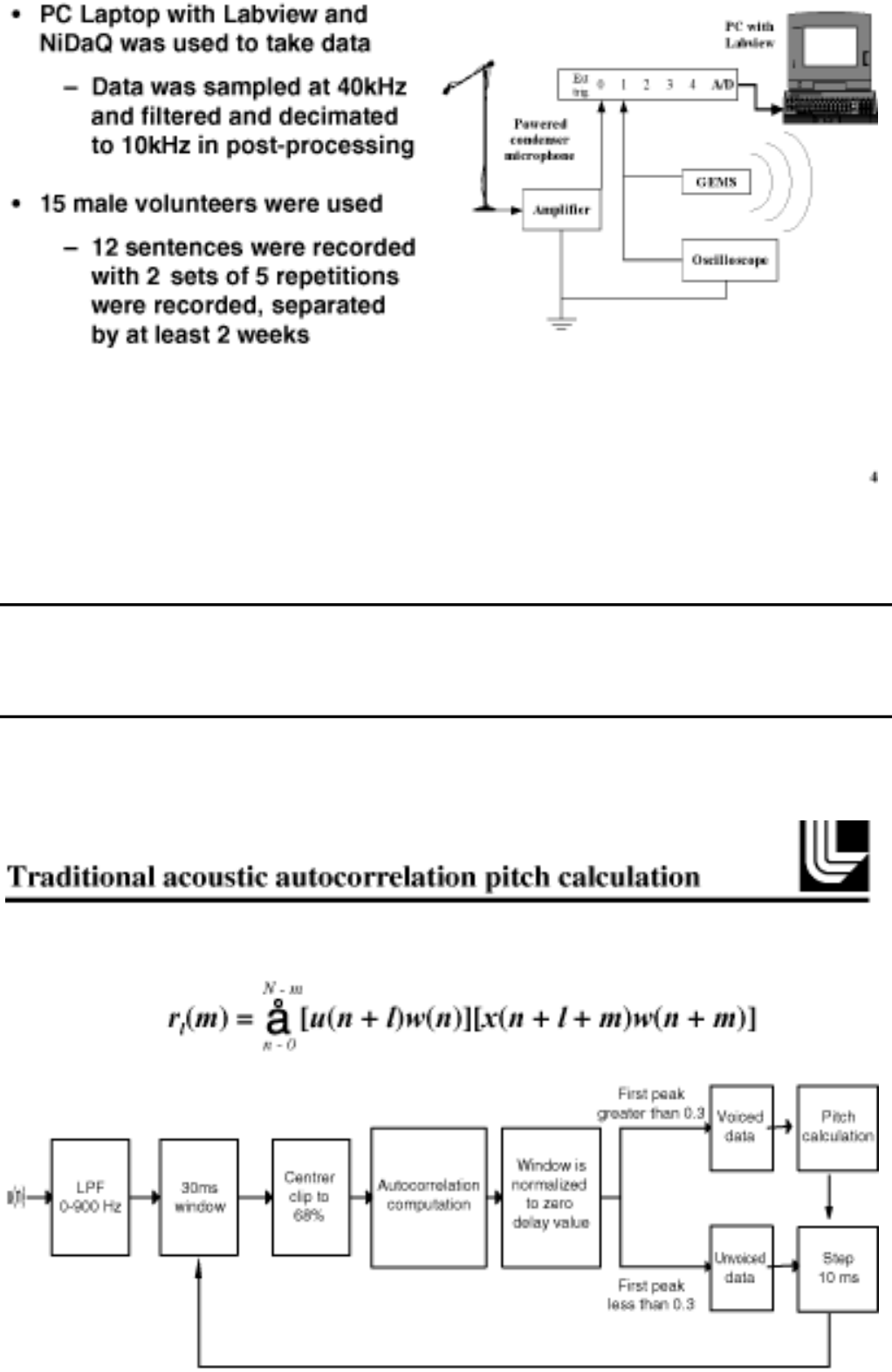


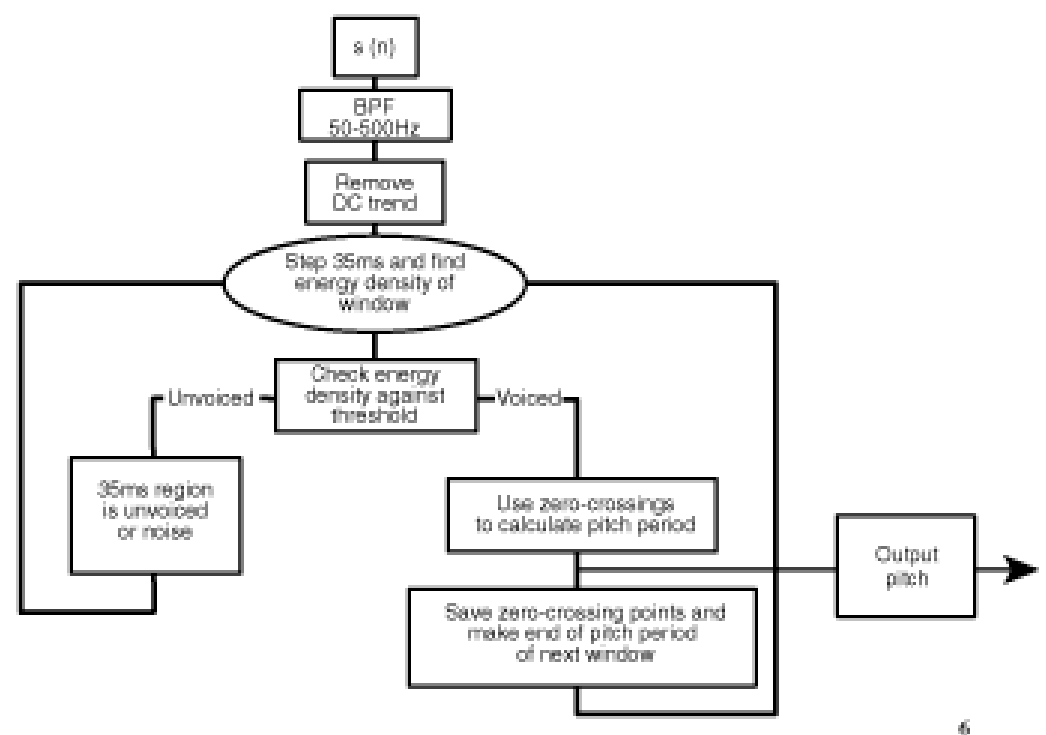

Pitch extraction performance comparison

Pick $f_{k}$ at random to four decimal places

Synthetic signal $\quad \mathrm{s}(\mathrm{t})=\underset{\mathrm{N}=1}{\mathrm{a}} \frac{1}{\mathrm{~N}} \sin \left(2 \pi N \mathrm{f}_{\mathrm{k}} \mathrm{t}\right)$

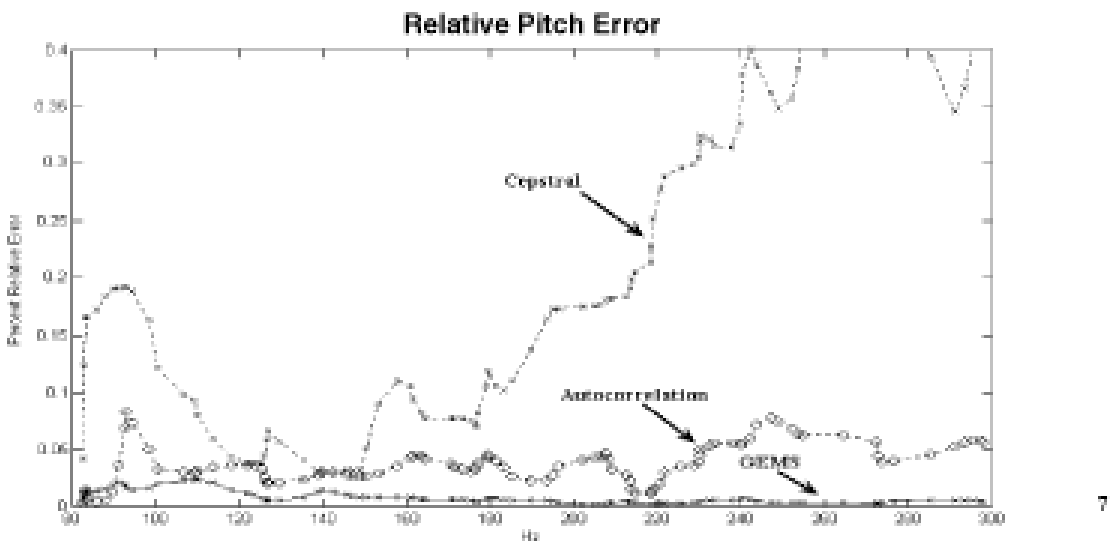


Example of showing that GEMS' pitch estimate is unaffected by acoustic noise

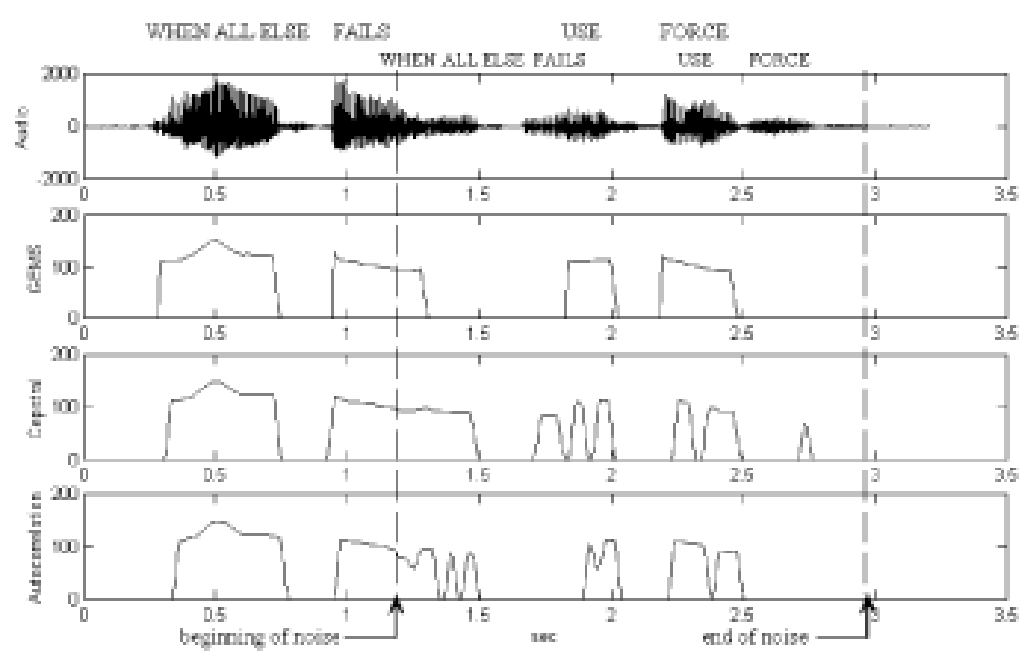

From Tuning Fork Experiments We Determine Pitch Estimate Performance of the GEMS

- Generate known signals from tuning fork experiments

- Compute pitch estimate using cepstral, autocorr, and GEMS zero-crossing

- Results indicated that GEMS pitch estimate is:

- 100 times faster in computational efficiency

- $\mathbf{5}$ to 20 times smaller in error rates

- Conclusions

- GEMS is insensitive to acoustic noise

- Fast computation of pitch can provide real-time pitch synchronous speech processing

- Performance improve with higher sampling rate

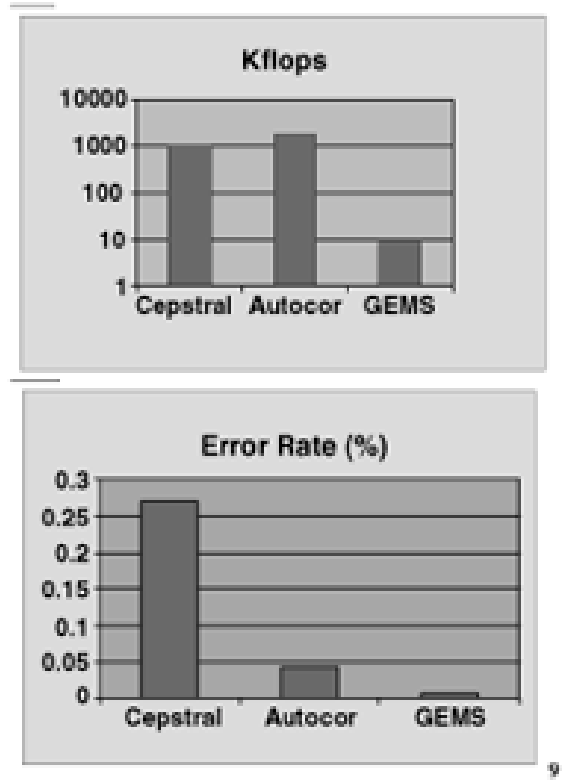


Speaker verification is the process of accepting or rejecting a persons identity claim based on parameters extracted from a test utterance

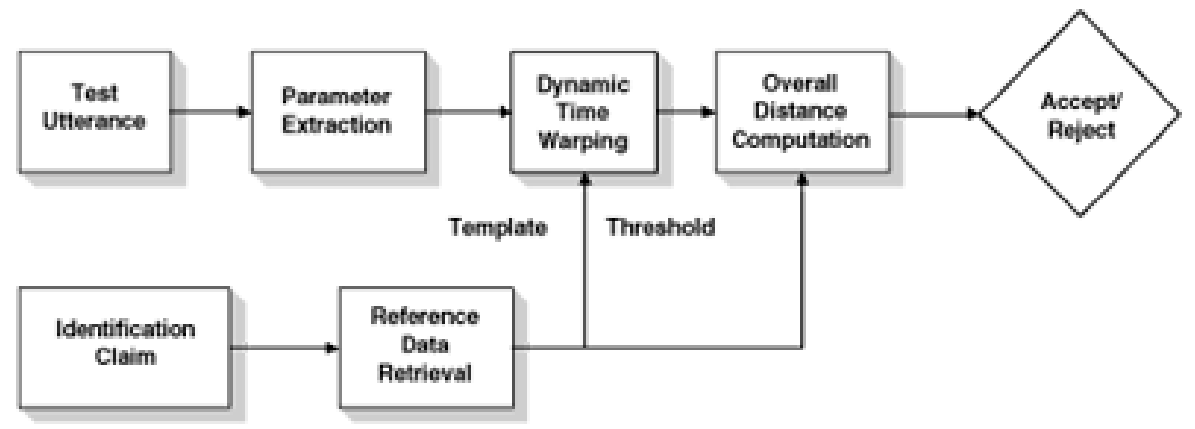

Dynamic Time Warping (DTW) is the process of warping two vectors to the same length which yields a distance measure that mathematically tells us how similar they are
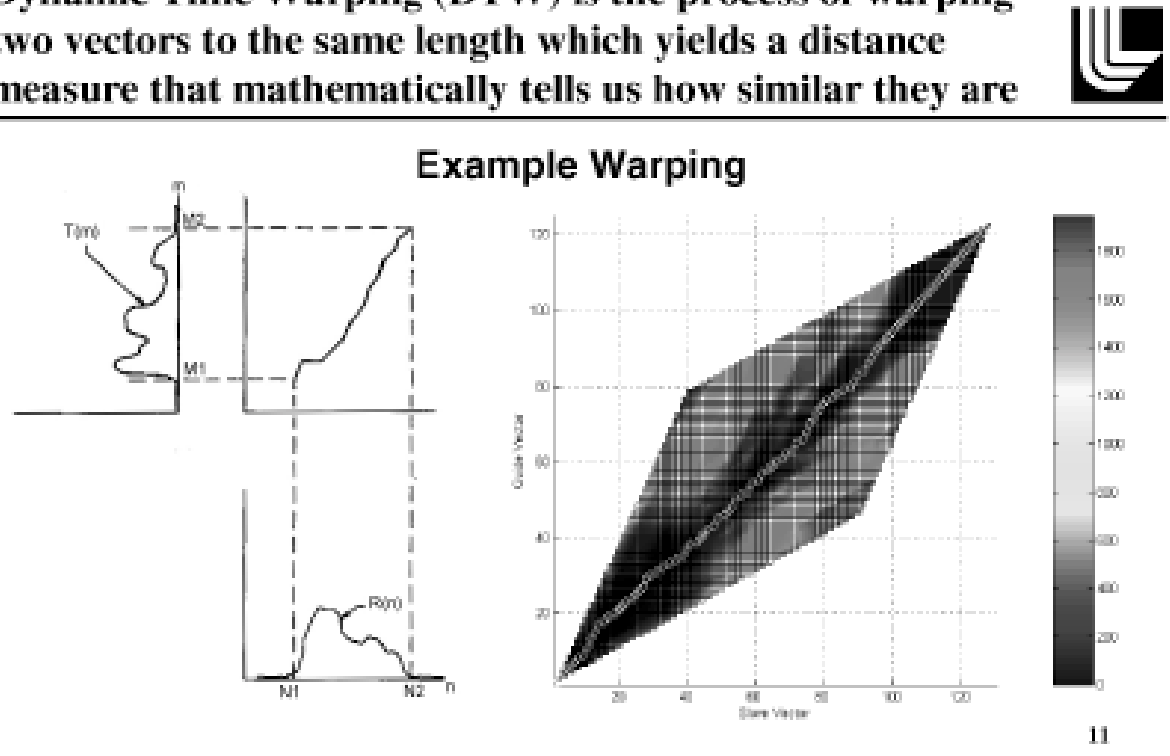
How to find the Equal Error Rate (EER) from the warping distances

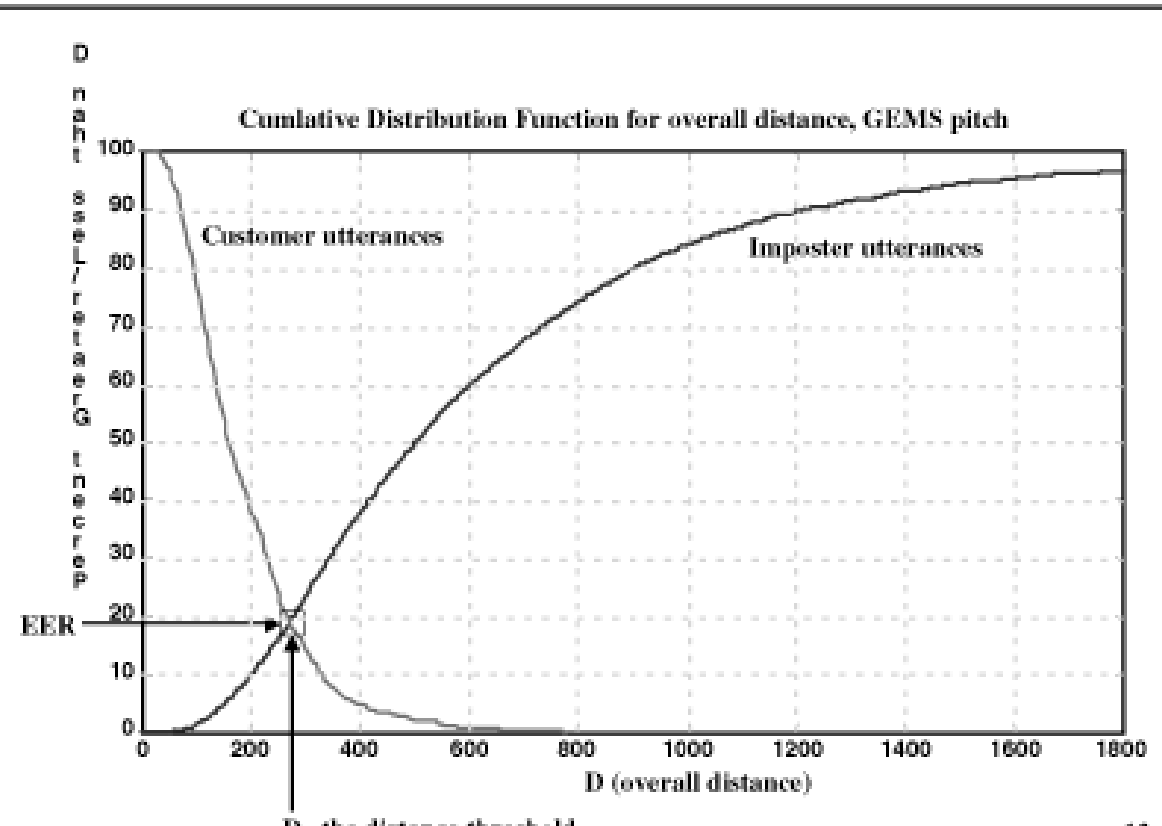

GEMS equal error rate (EER) is insensitive to acoustic noise
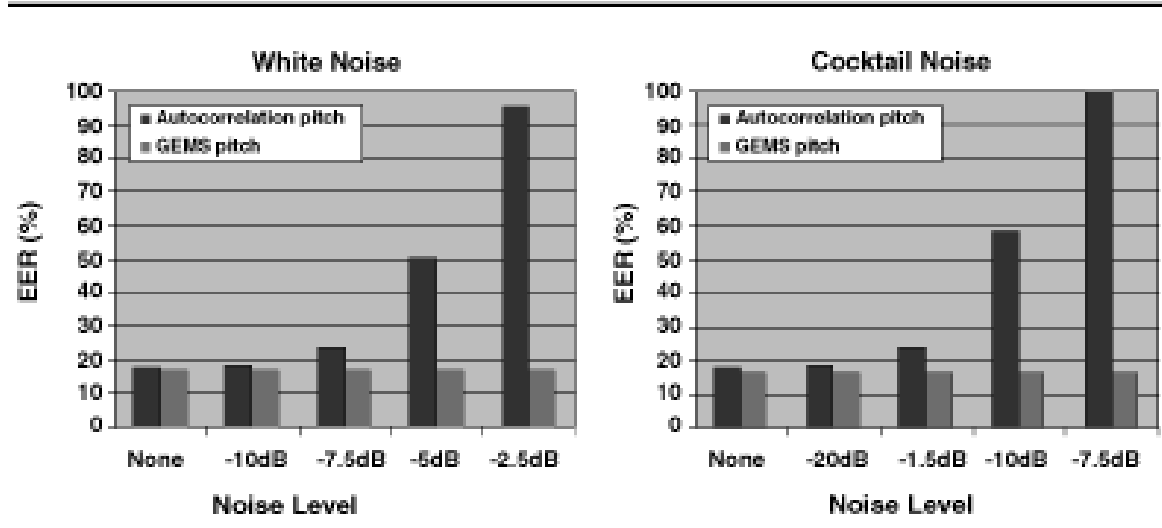


\section{Conclusions}

- The GEMS is a small non-invasive, low power, low cost device well suited for pitch extraction

- The GEMS sensor is fast, accurate and noise insensitive

- With this more accurate and robust pitch extraction method, pitch can be used more reliably in speaker verification

- With the present database, the GEMS extracted pitch based verification is about $10 \%$ better than acoustic autocorrelation pitch based verification. Additional EM sensor based features will further reduce verification error rates. 


\title{
Defining a Human Voiced Excitation Function using Glottal Electromagnetic Micropower Sensors (GEMS)
}

\author{
T.J. Gable, L.C. Ng, G.C. Burnett, and J.F. Holzrichter \\ Lawrence Livermore National Laboratory, POB 808, Livermore CA 94551
}

This work compares the speaker verification performance between a traditional acoustic-only pitch extraction to a new electromagnetic (EM) sensor based pitch approach. The pitch estimation approach was developed at the Lawrence Livermore National Laboratory (LLNL) utilizing Glottal Electromagnetic Micropower Sensors (GEMS, also see http: / / speech.llnl.gov/). This work expands previous pitch detection work by Burnett et. al. (to be published IEEE Trans. on Speech and Audio Processing) to the specific application of speaker verification using dynamic time warping. Clearly, a distinct advantage of GEMS is its insensitivity to acoustic ambient noise. This work demonstrates the clear advantage of the GEMS pitch extraction to improve speaker verification error rates. Cases with added white noise and other speech noise were also examined to show the strengths of the GEMS sensor in these conditions. The EM sensor speaker verification process operated without change over signal-to-noise (SNR) conditions ranging from $-20 \mathrm{~dB}$ to $-2.5 \mathrm{~dB}$, the acoustic algorithms became unusable at SNR exceeding $-10 \mathrm{db}$. [Work supported by NSF and DOE.]

\section{Defining a Human Voiced Excitation Function using Glottal Electromagnetic Micropower Sensors (GEMS)}

\section{난 \\ CASIS Signal and Image Sciences Workshop}

November 11, 1999

Greg Burnett

Lawrence Livermore National Laboratory 


\section{Glottal EM sensor tissue measurements}

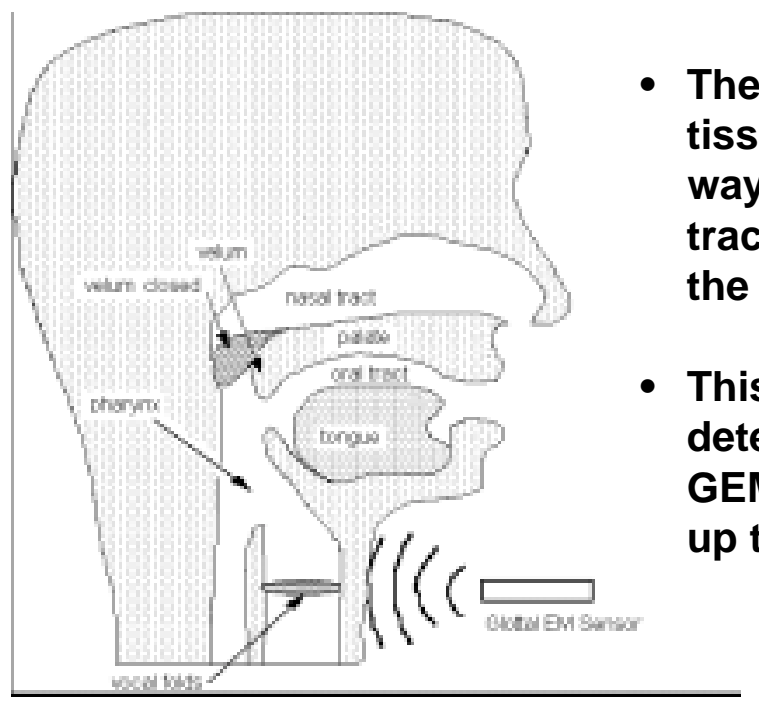

\section{Raw GEMS signal coming from neck}
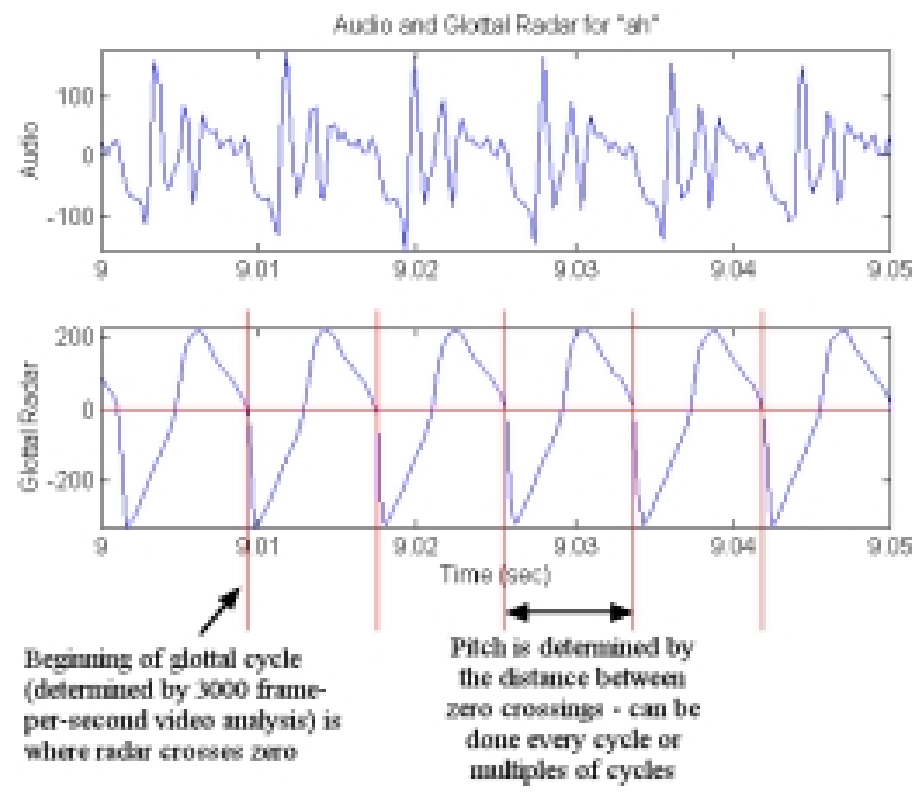
Using noncausal digital filters, we now can calculate position and velocity for objects moving with frequencies above about $75 \mathrm{~Hz}$

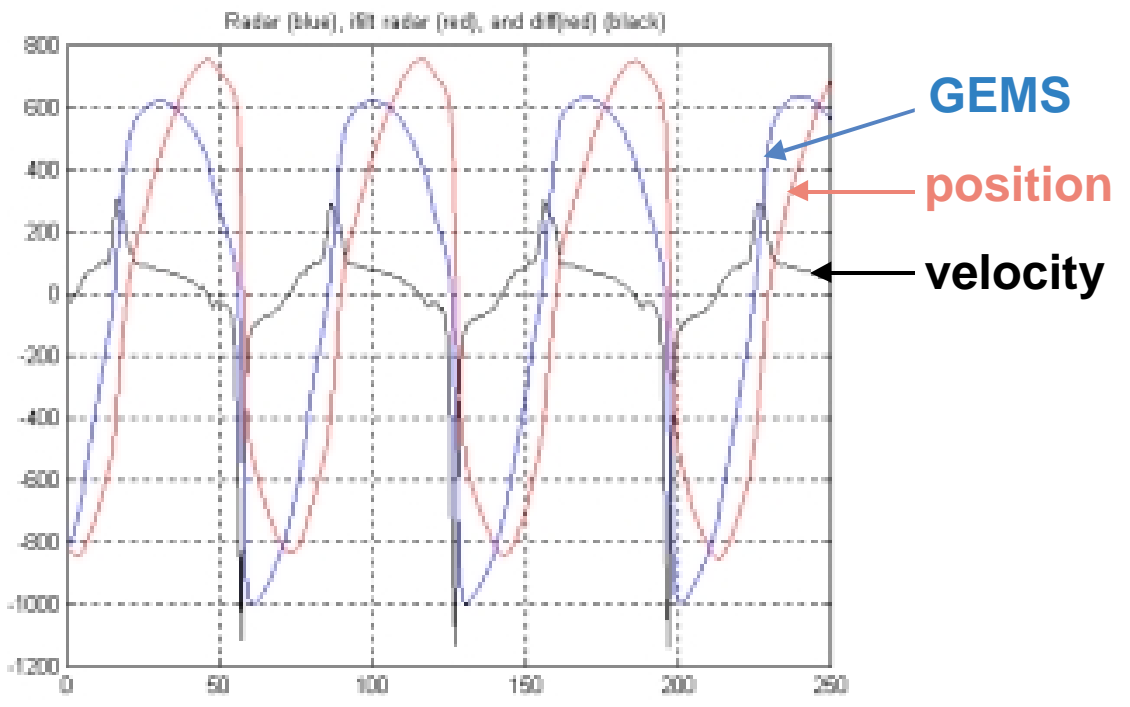

\section{Anatomy suggested that the posterior tracheal wall} would be a better reflector than the anterior wall or folds

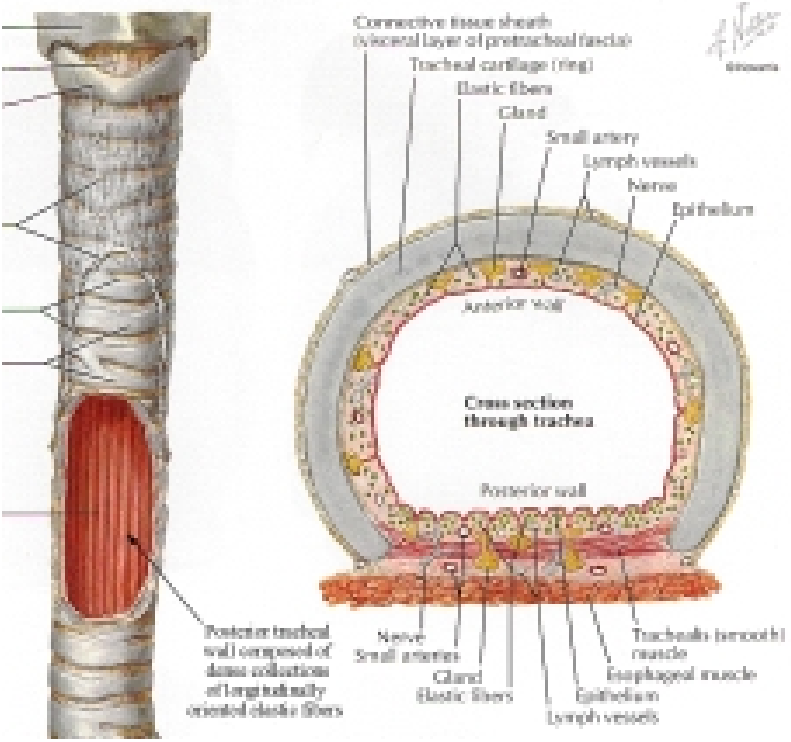

The posterior wall is flat and thick but quite flexible, while the folds do not present a significant scattering area to the GEMS

Copyright 1989. Novartis. Reprinted with permission from the Atlas of Human Anatomy, illustrated by Frank H. Netter, M.D. All rights reserved. 
Electromagnetic simulations using Jeff Kallman's TSARLITE indicate the trachea reflects far more energy than the max-open folds

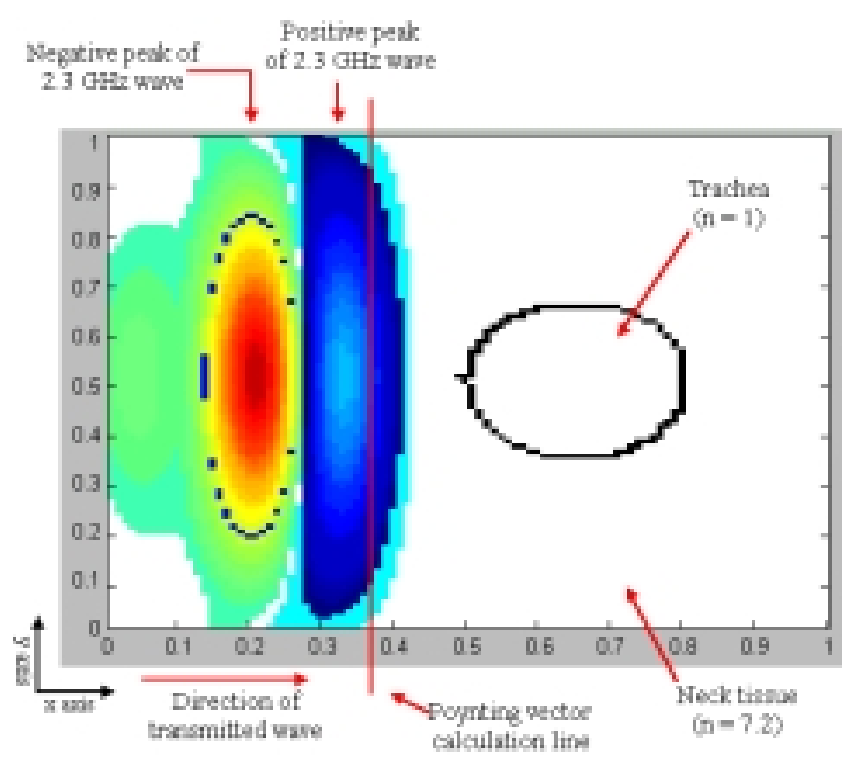

Trachea had a reflectivity of $15.2 \%$, more than $18 x$ the max-open fold reflectivity of $0.8 \%$ (close to the noise level of the simulation)

High speed (1000-3000 fps) video experiments indicated fold motion did not correlate well with GEMS signal

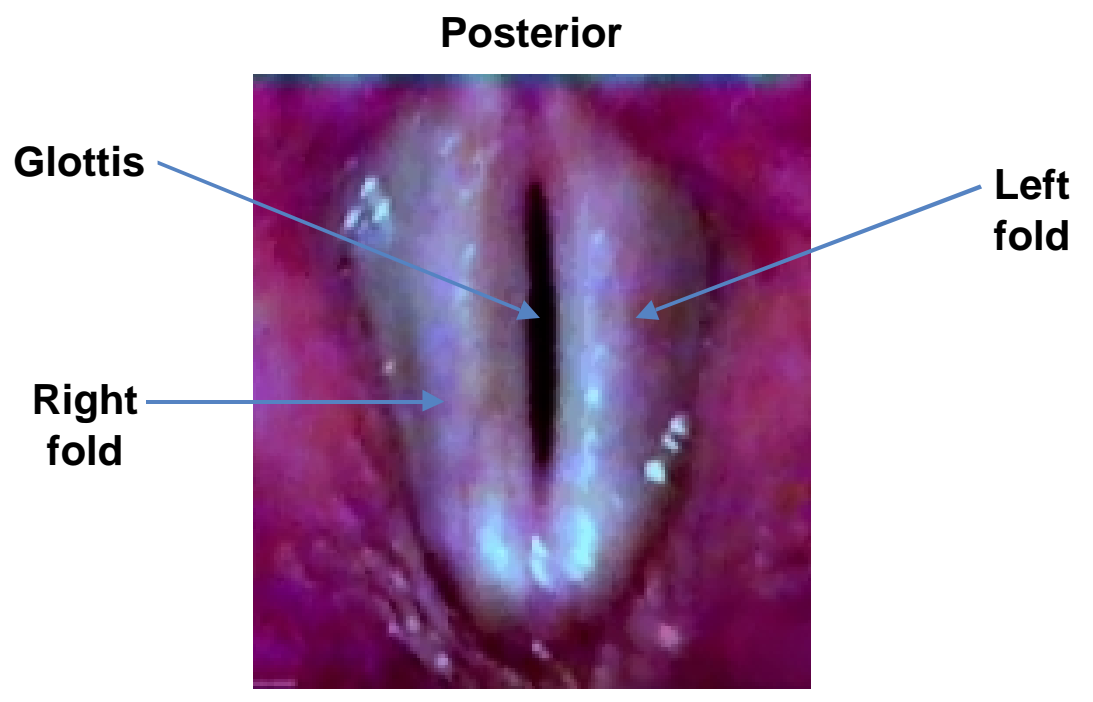

Anterior 
Conclusions about physiological basis of the GEMS return

- The object responsible for the detected vibration is the subglottal posterior tracheal wall

- This wall is driven by the changes in subglottal air pressure as voicing occurs

- To define an excitation function for the human vocal tract we must relate the detected tracheal motion to the driving subglottal pressure

Using the excitation function as the input to an LTI system we can calculate the transfer function of the vocal tract

\section{Pressure}

signal

x(t)

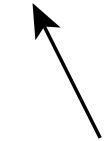

Excitation

function

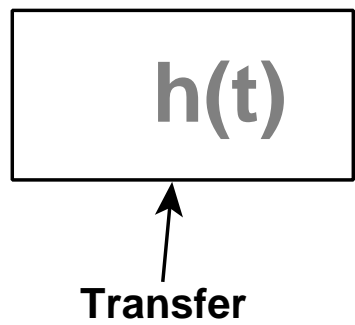

function

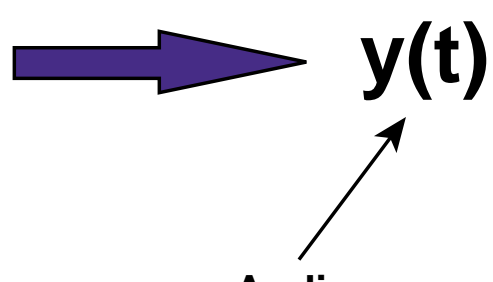

Audio 


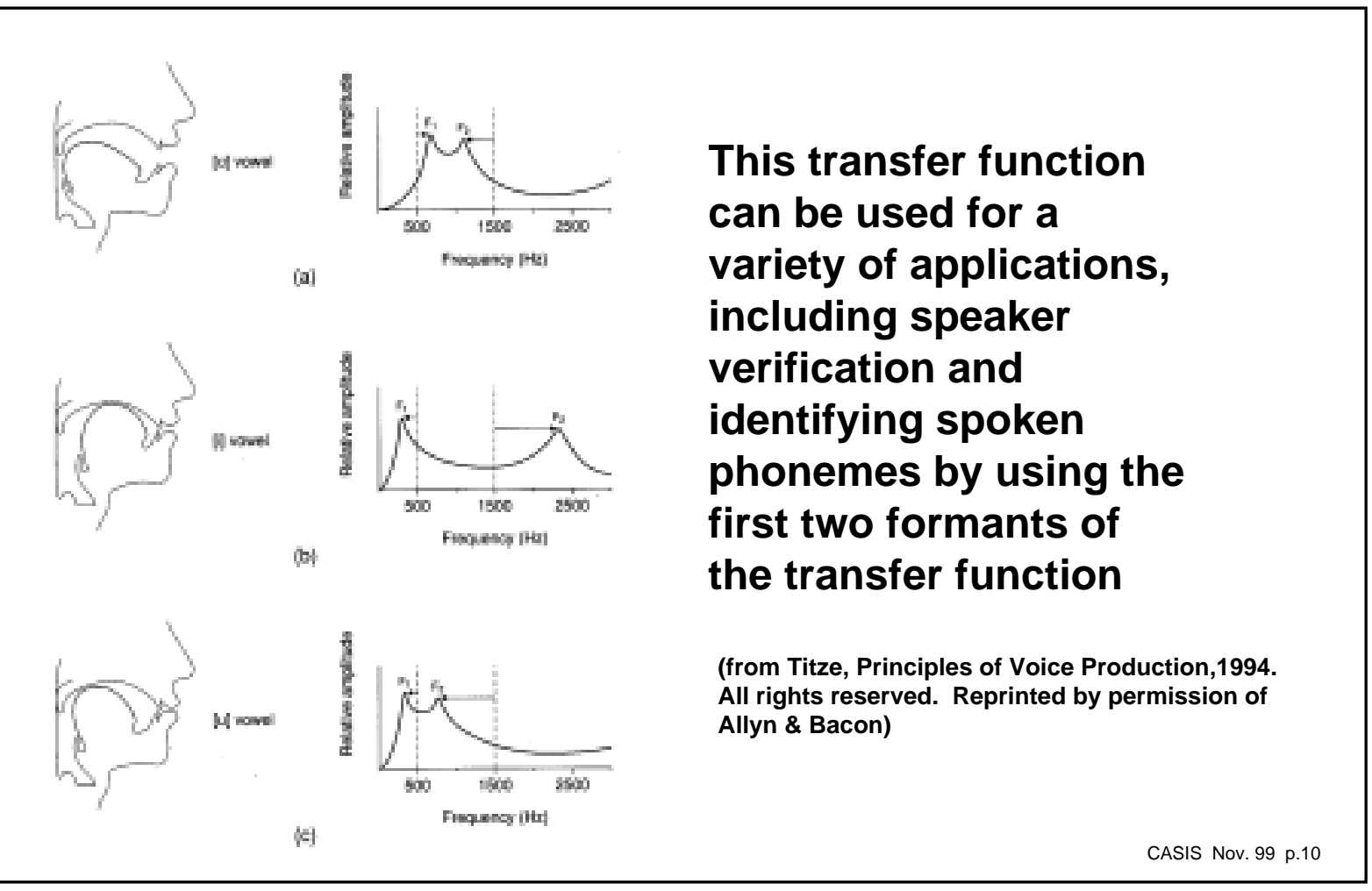

\section{A lumped-element circuit model is used to quantify the trachea's response to changing air pressure}

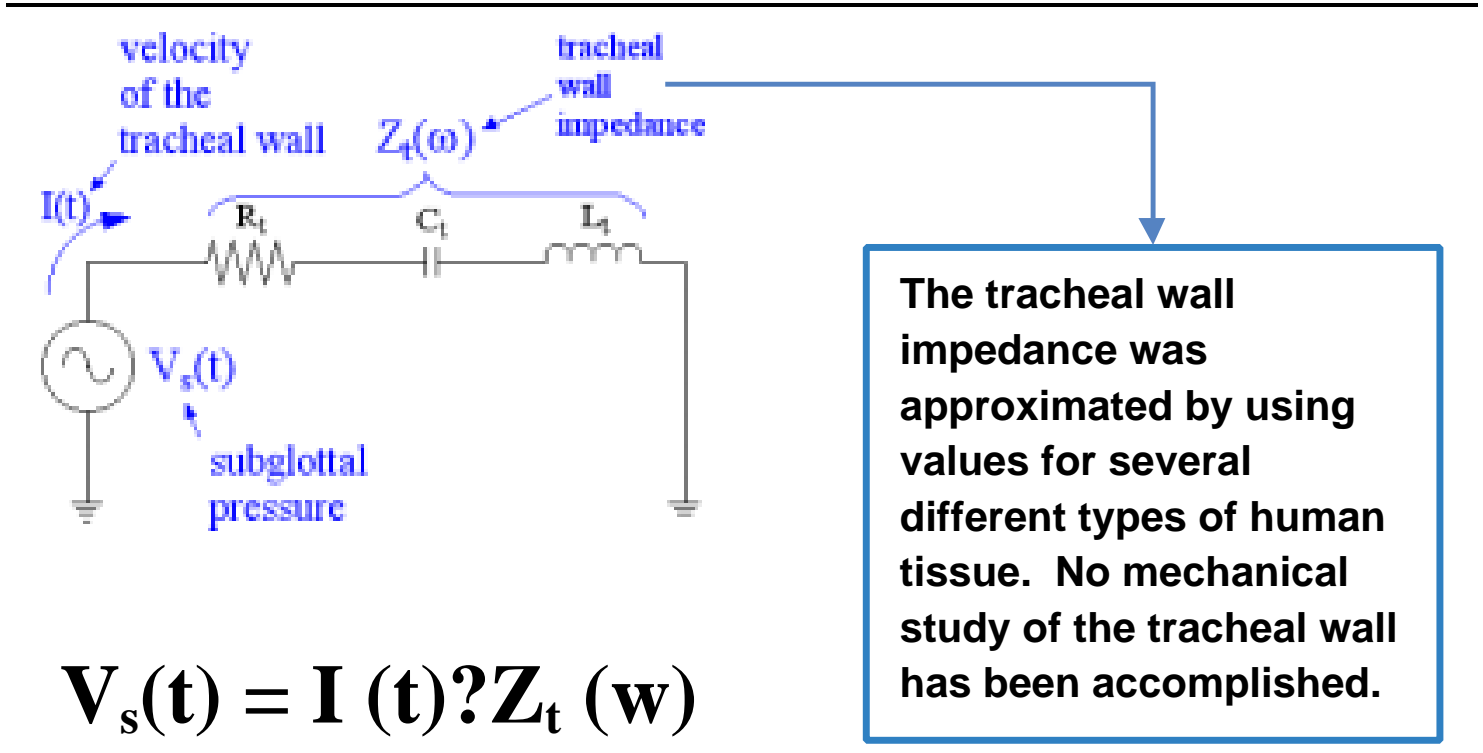


The resulting impedance frequency response is modeled with a digital lowpass and three anticausal allpass filters
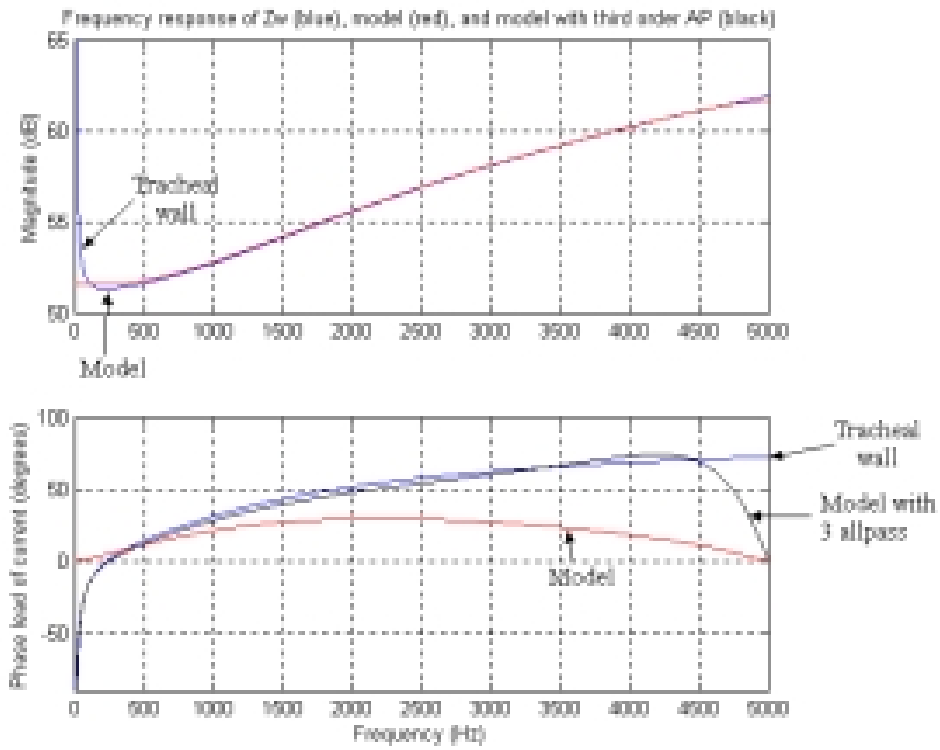

The resulting digital filter can then be used to filter the calculated wall velocity to recover the driving subglottal pressure (black trace)

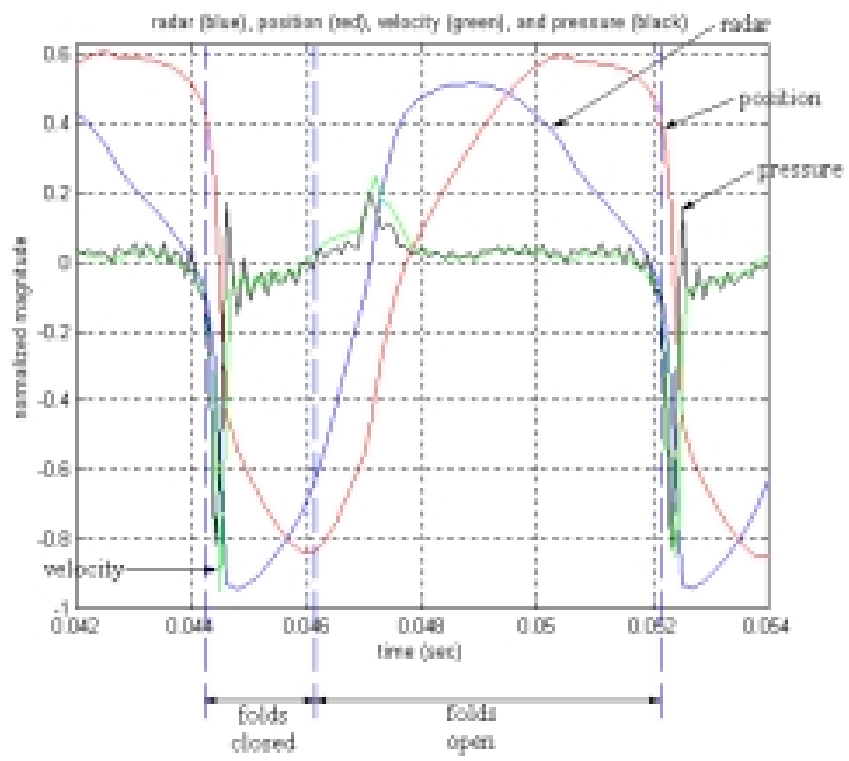


Using the excitation function as the input to an LTI system we can calculate the transfer function of the vocal tract

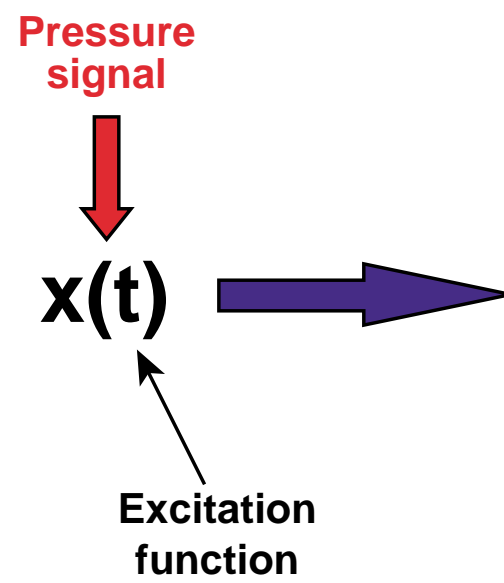

Example of the calculated TF for an "ah"

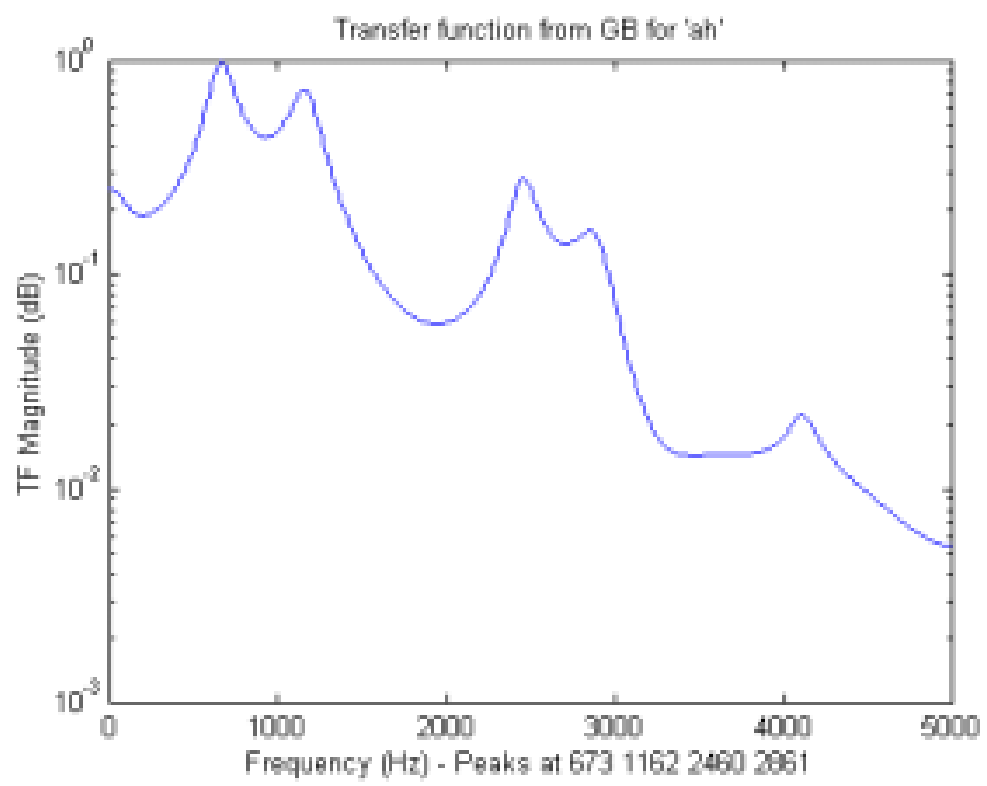




\section{Example of the calculated TF for an "ee"}

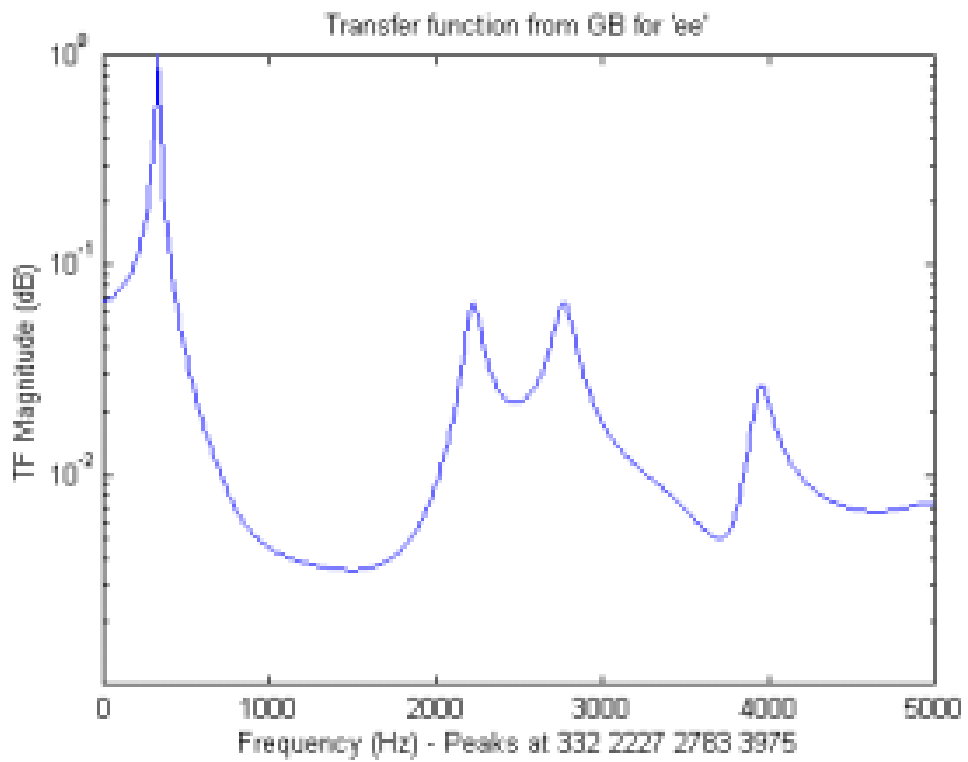

CASIS Nov. 99 p.16

\section{Example of the calculated TF for an "oo"}

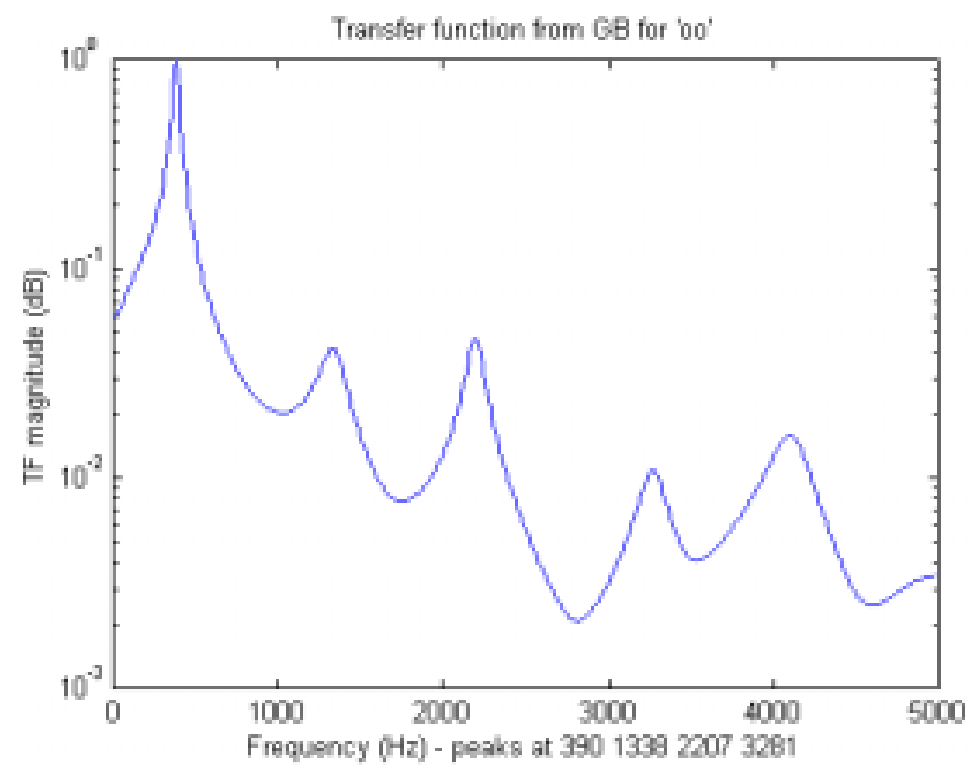




\section{In conclusion}

- The GEMS is an excellent vibration detector that can detect the motion of the rear tracheal wall during voiced speech

- This position of the rear wall may be differentiated and filtered using a lumped element model of the wall in order to derive the subglottal pressure

- This subglottal pressure may be used as the excitation function for the vocal tract

- With the excitation function, the transfer function of the vocal tract can be calculated

- The transfer function can be useful in phoneme identification and speaker verification 


\title{
Synapse TAP ${ }^{T M}$ Universal Access Technology
}

\author{
Marty Tibor, Synapse \\ Speech Recognition and Adaptive Technology
}

The Synapse TAP ${ }^{\mathrm{TM}}$ provides continuous speech recognition on computers regardless of platform. The TAP ${ }^{\mathrm{TM}}$ is a universal access technology developed in collaboration with Stanford University.

1. The user dictates text and commands at speeds up to 160 words per minute into the speech-accessing device.

2. The speech accessor converts in real-time the users' speech into keystrokes, commands and mouse events. The accessor sends its data stream to the Synapse TAPTM module.

3. The TAPтм module then converts the events in real-time into the native format of the target machine.

Presently TAPTM modules support any operating system or application found on HP, Sun and SGI UNIX workstations, Mac and IBM personal computers and any mainframe than can support a PC or Mac terminal through 3270 or other emulation. Besides providing continuous speech recognition on platforms not currently supported the TAP devices have these other features:

- Users may control with a single speech accessor up to four computers at once even if the target machines are on different hardware or software platforms.

- TAP technology collaborates numerous input technologies and therefore a user can deploy speech recognition, head tracking, single switches, foot pedals or mouse at the same time without clogging the input stream of the target computer.

- A universal command structure can easily be developed using TAP ${ }^{\mathrm{TM}}$ technology where all computers can respond to exactly the same commands. For example dictating "print document" can mean the same thing to all target computers regardless of platform or application. Training time can be greatly reduced since a user would only need to become familiar with one command structure for all environments.

Presently TAPTM technology is used a productivity tool for data entry, dictation and controlling graphical applications such as mapping, CAD and modeling. TAP ${ }^{\mathrm{TM}}$ technology is also deployed to support users with repetitive strain injuries or other disability that use advanced computer workstations. 


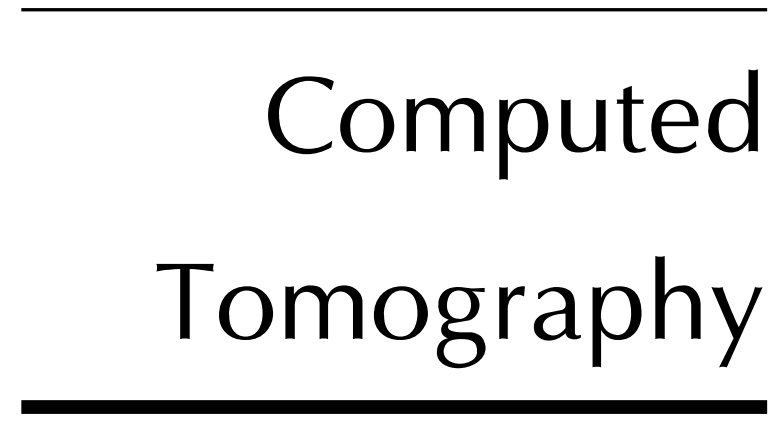




\section{Confocal Ultrasound Imaging Through Acoustically Thick Media}

\section{UCRL-JC-136318 Abs}

Sean K. Lehman, David D. Scott, Waleed S. Haddad

To date, all confocal applications, whether optical or acoustic have been through optically or acoustically, respectively, thin media. We have developed a theory of transmission mode confocal imaging through thick media. We have used a full finite difference time domain simulation of acoustic field scattering through a medium to demonstrate a proof-of-principle for the theory. We present preliminary results based upon these simulations.

\section{Confocal Ultrasound Imaging Through Acoustically Thick Media}

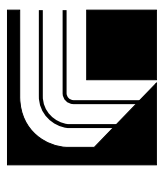

Sean K. Lehman

David D. Scott

Waleed S. Haddad

David H. Chambers

CASIS Workshop

November 11, 1999 


\section{Introduction}

- Definition of Problem

- Problem Domain

- Scanning Technique

- Simulated Examples

- Current Results

\section{Definition of Problem}

- Perform an acoustic confocal scan of an object and construct an image of the object using tomographic techniques;

- Assume low sound speed contrast, i.e. small changes in refractive index.

We hope to achieve higher contrast reconstructions over traditional straight ray tomographic techniques. 


\section{Problem Domain}
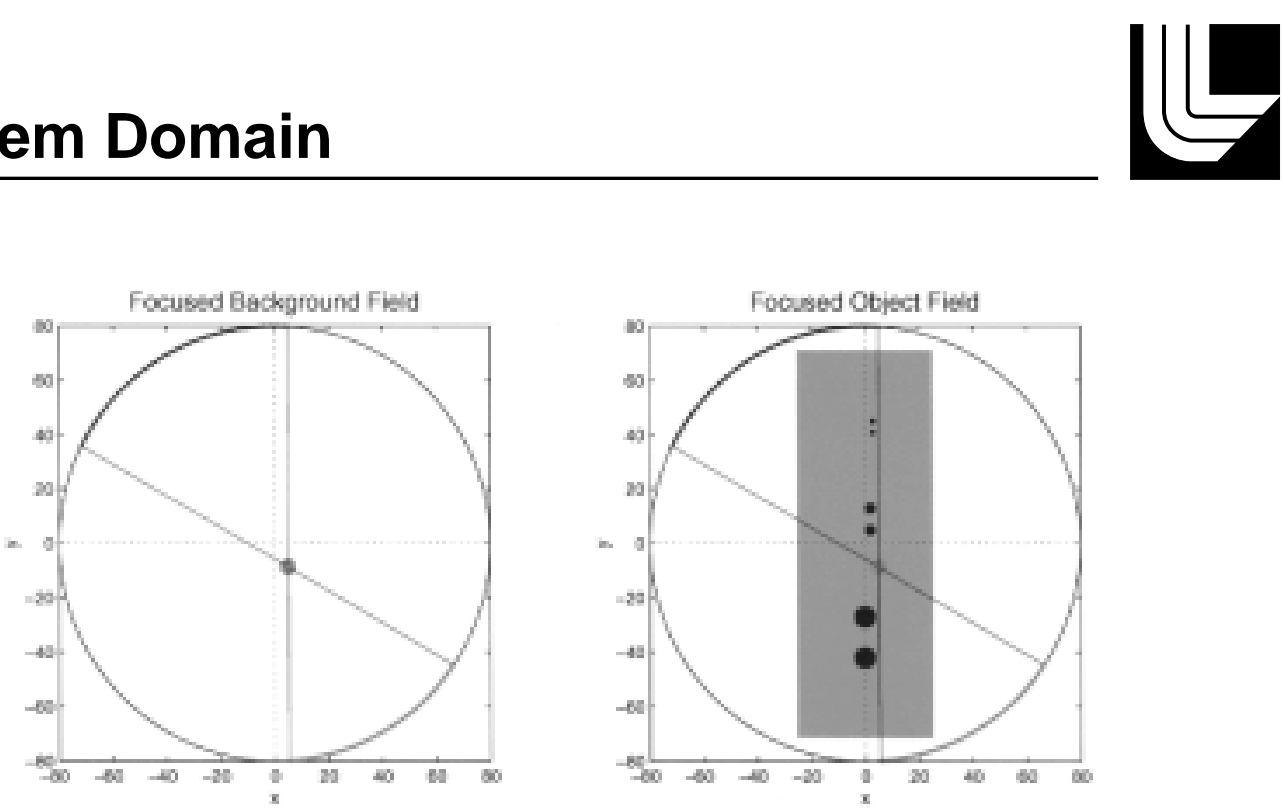

\section{Scanning Technique}

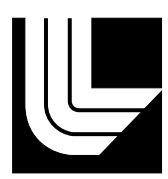

- Perform a scan through the object at the desired resolution. This is the inhomogeneous received field.

- Perform a background scan (in the absence of the object) at the same resolution. This is the homogeneous received field.

- At each focus, correlate the inhomogeneous received field with the homogeneous received field.

- Differences between the homogeneous and inhomogeneous received field amplitude and arrival times are related to the object's attenuation and sound speed at the focus..

We are developing a tomographic reconstruction algorithm which uses these measured differences to image the object's absorption and refractive index distribution. 


\section{Simulated Examples}

- Finite difference time domain simulation of object in a ring scanner with $\mathbf{3 6 0}$ equally spaced transmitters interleaved with 360 equally spaced receivers.

- $0.5 \mathrm{MHz}$

\section{Simulated Objects}
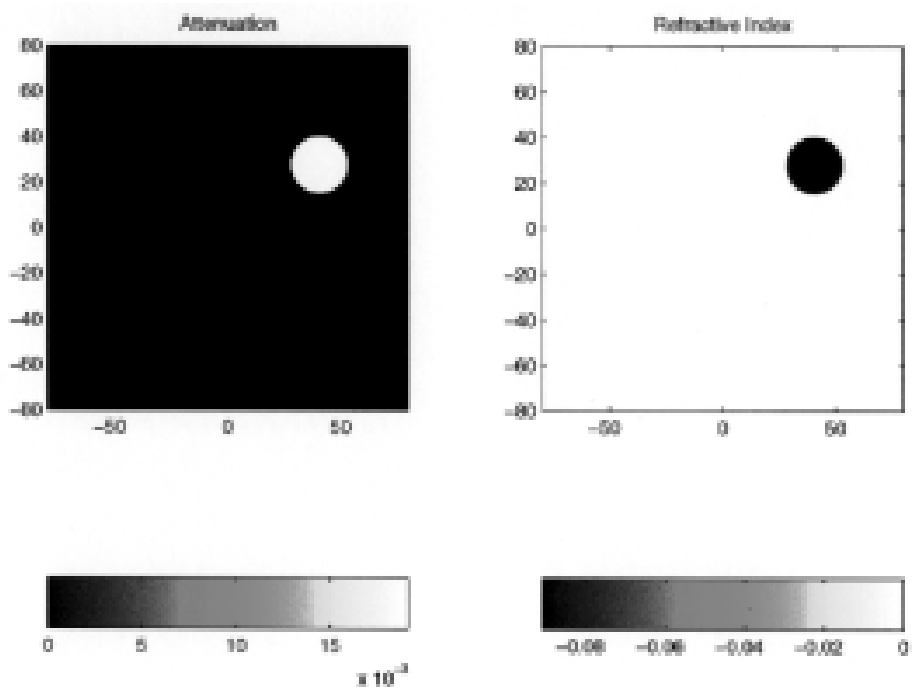


\section{Simulated Objects}
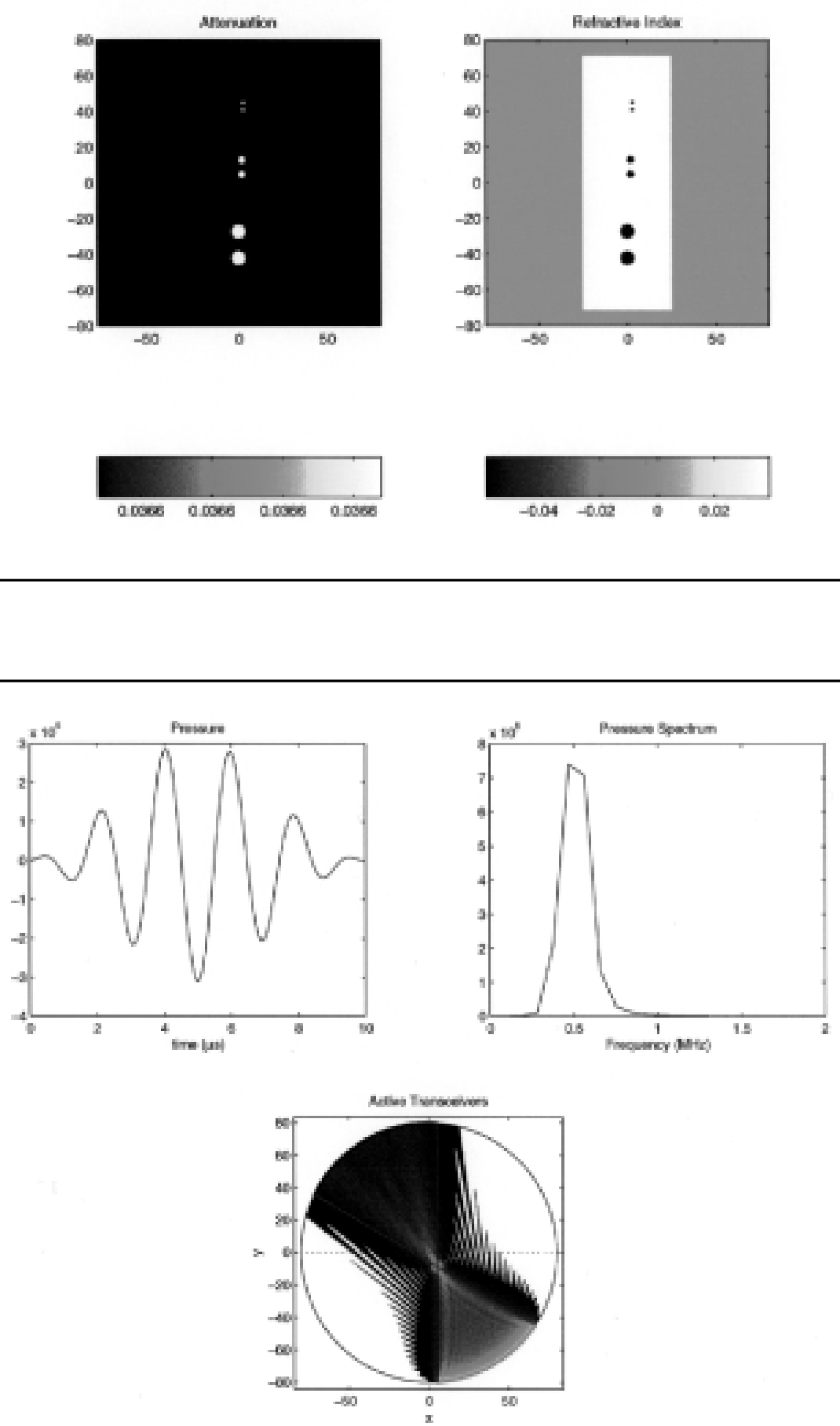

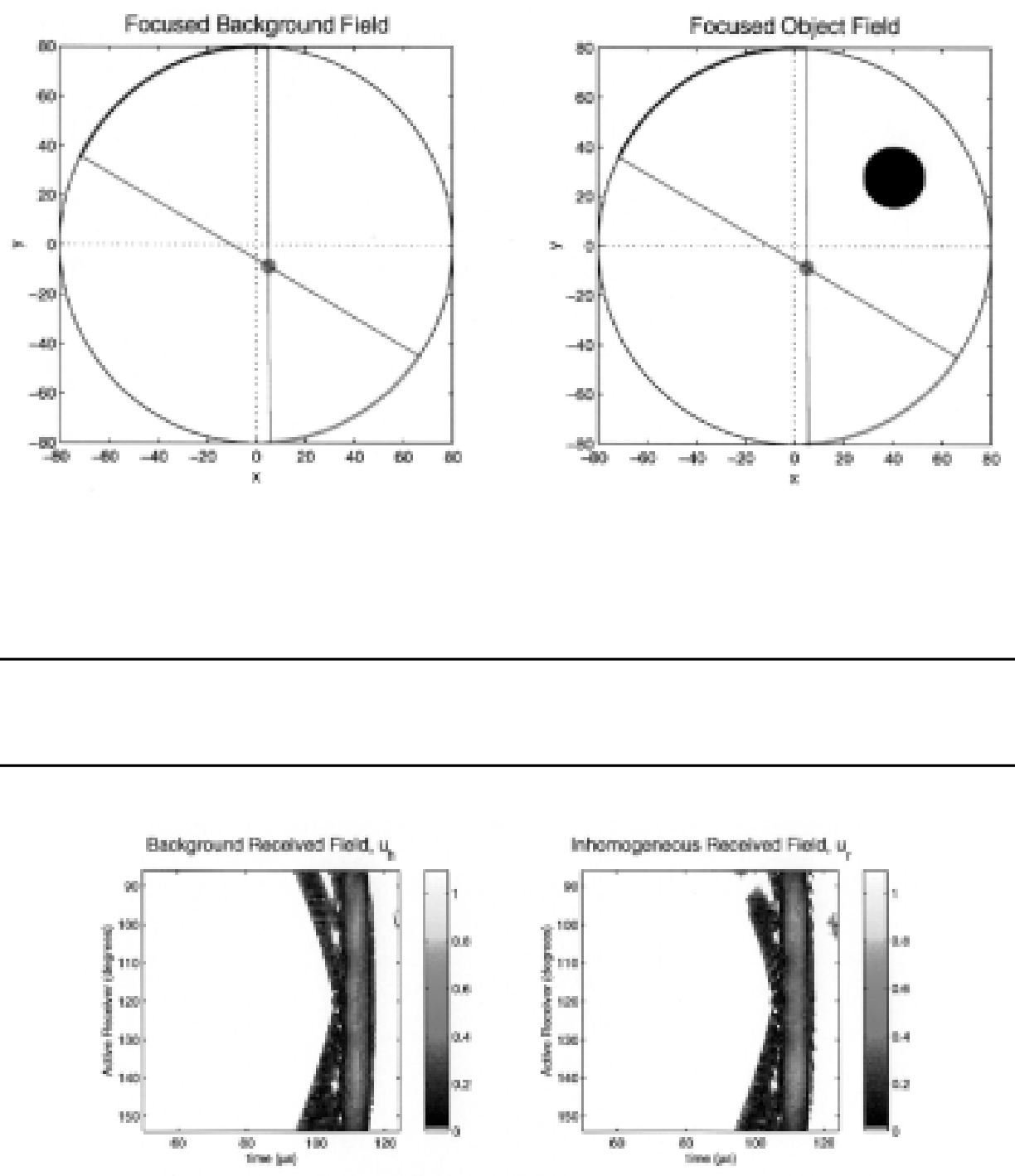

Crosscomelation, $R_{\text {h }}$

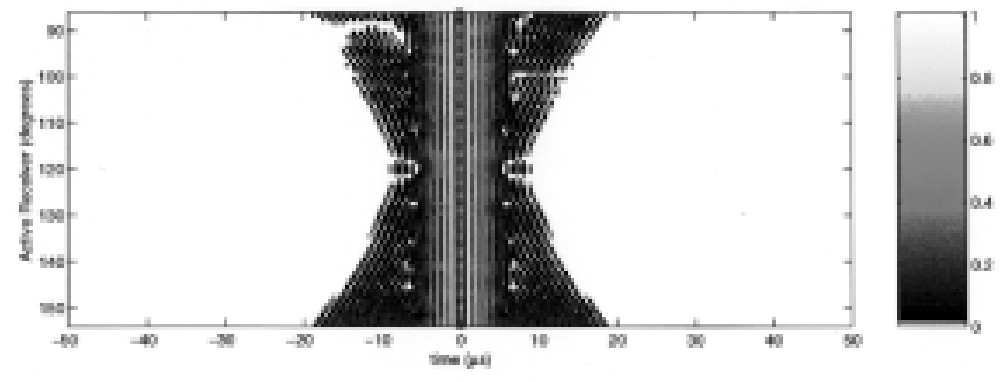




\section{O M P U T E D T O MO G R A P H Y}
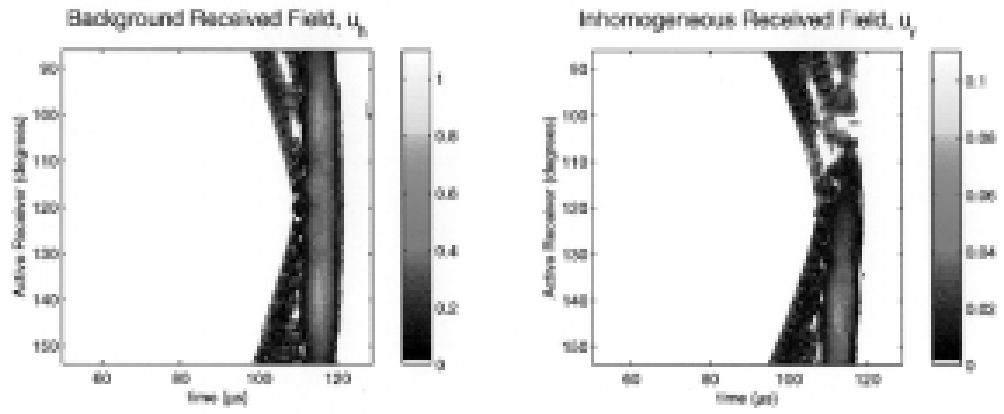

Crococomelation, $\mathrm{R}_{\mathrm{h}}$
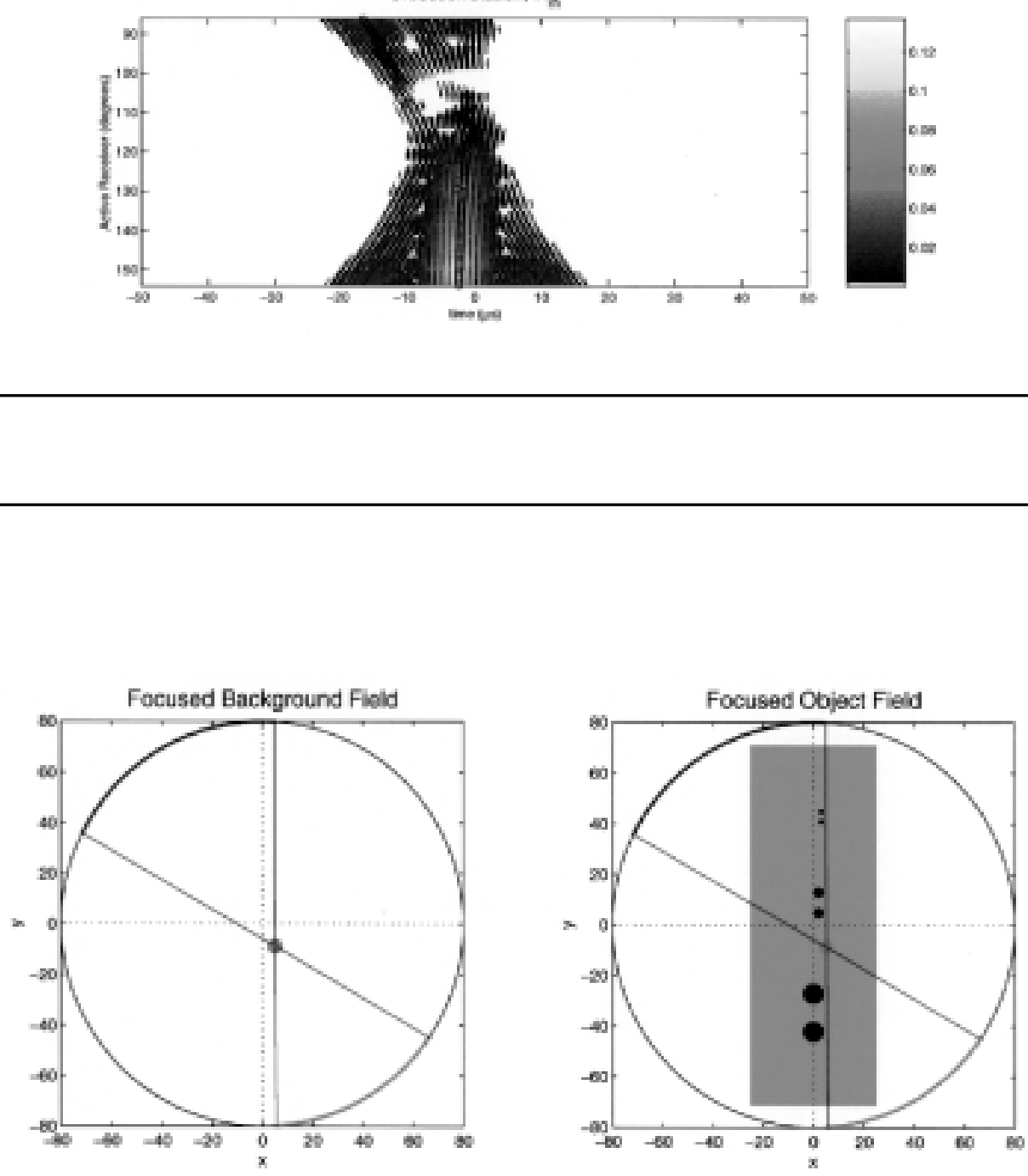


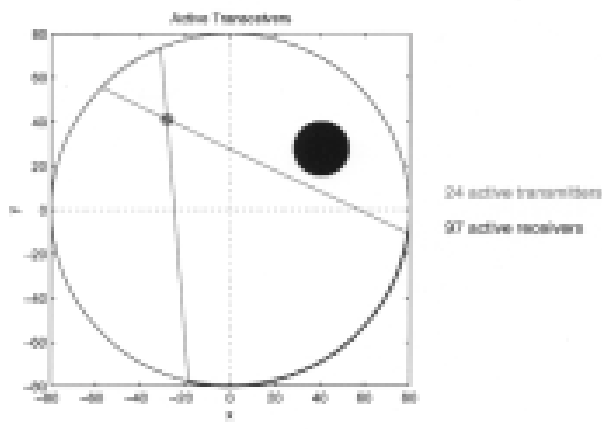

Foaried Ruceived Fild
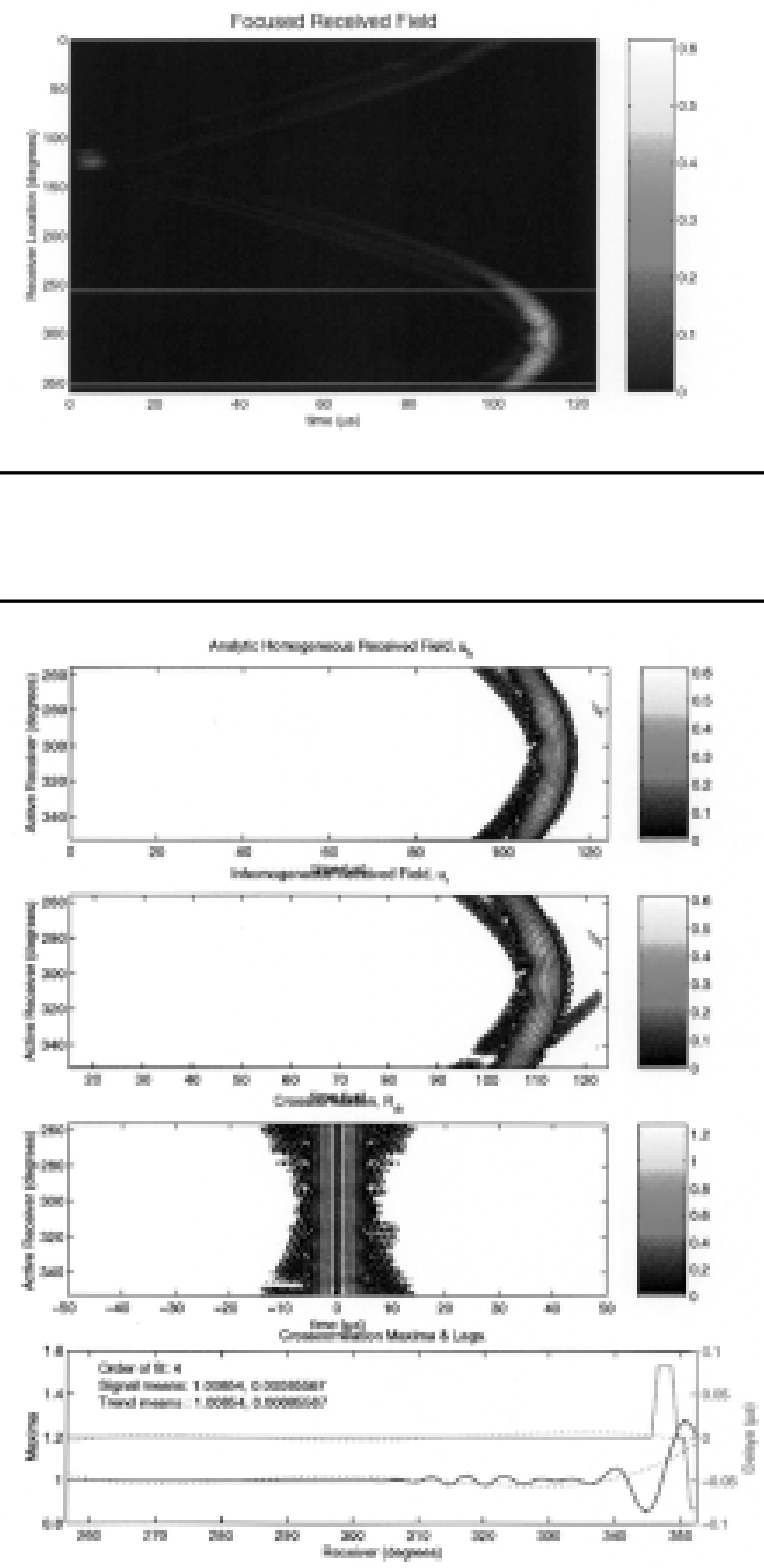

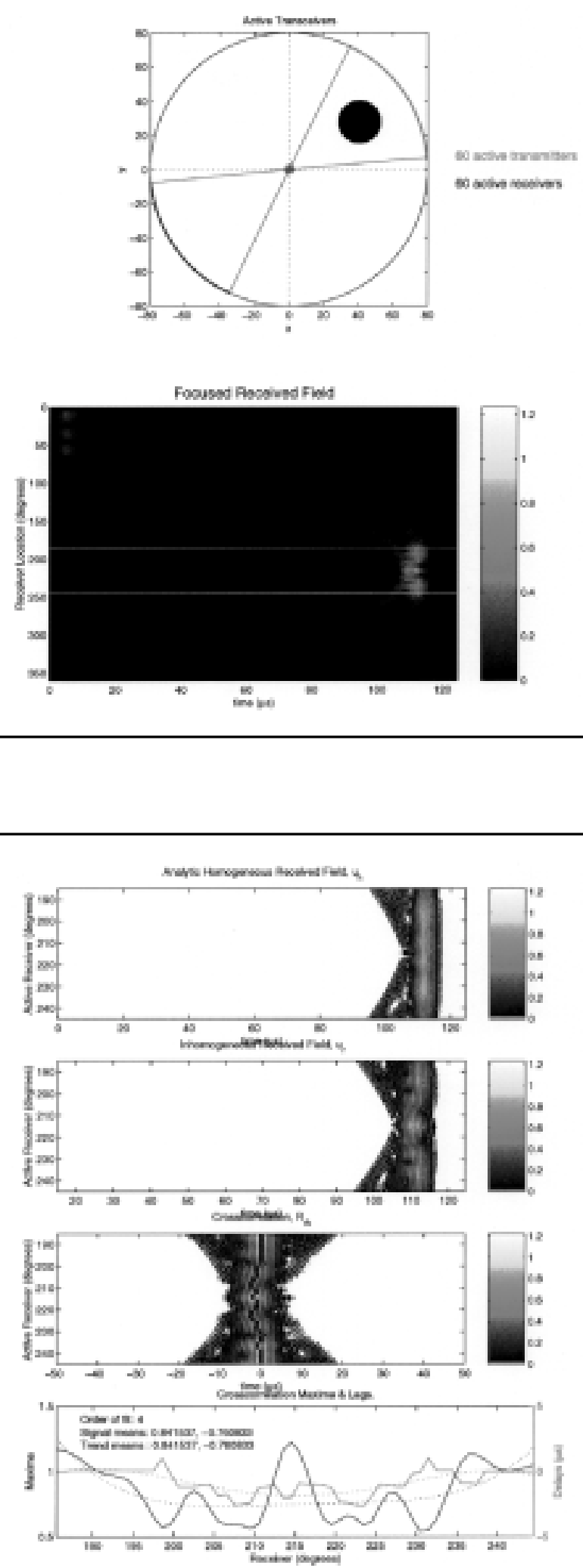
C O M P U T E D T O MO G R A P H Y

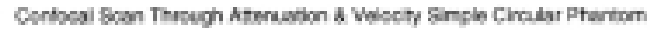
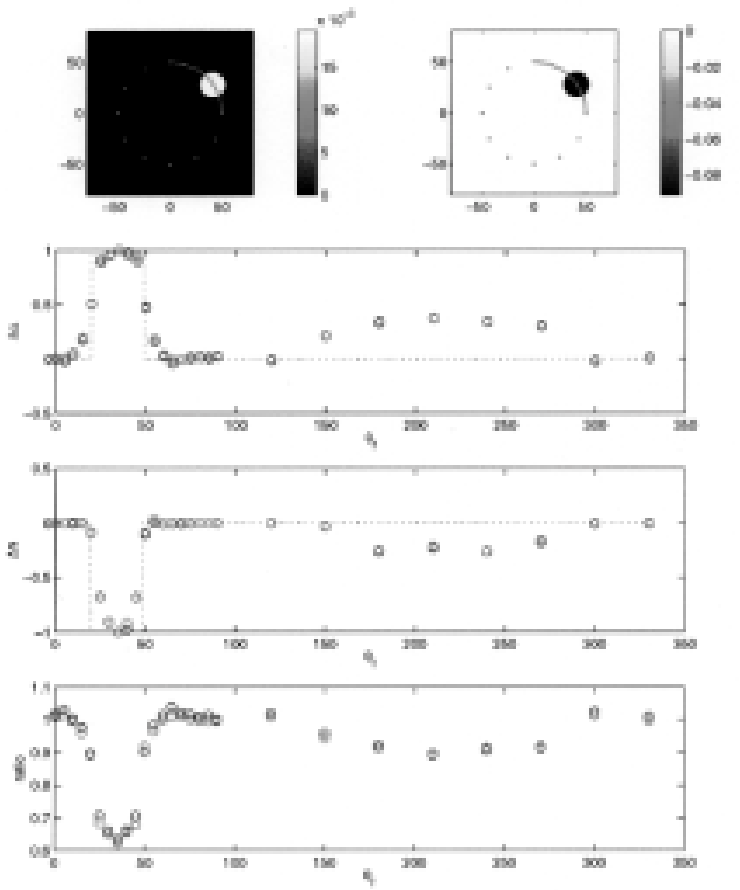

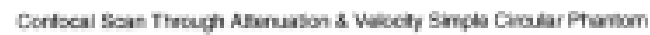
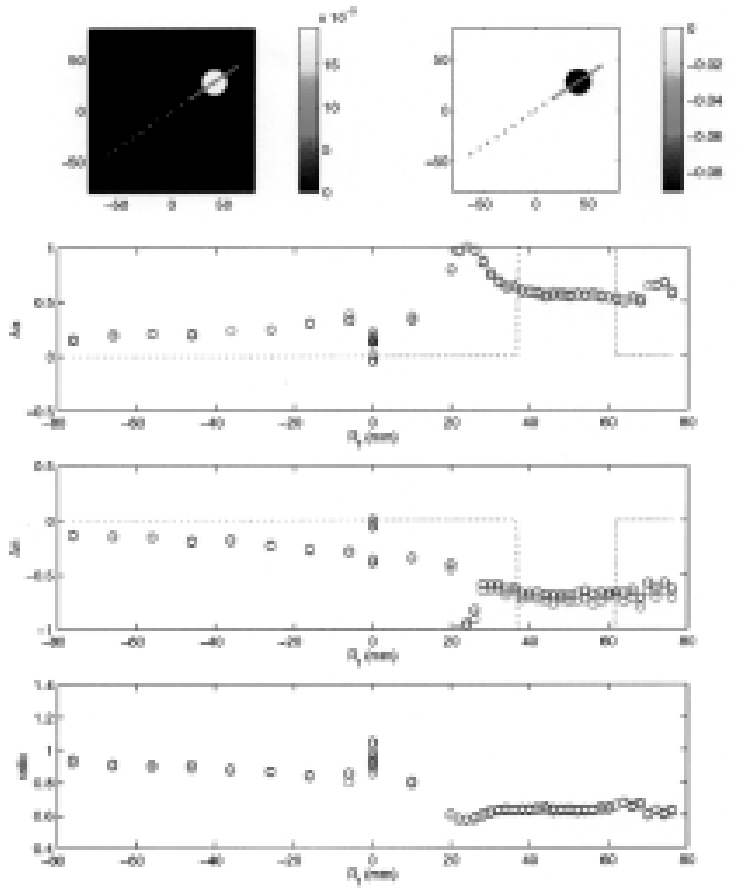
C O M P U T E D T O M O G R A P H Y
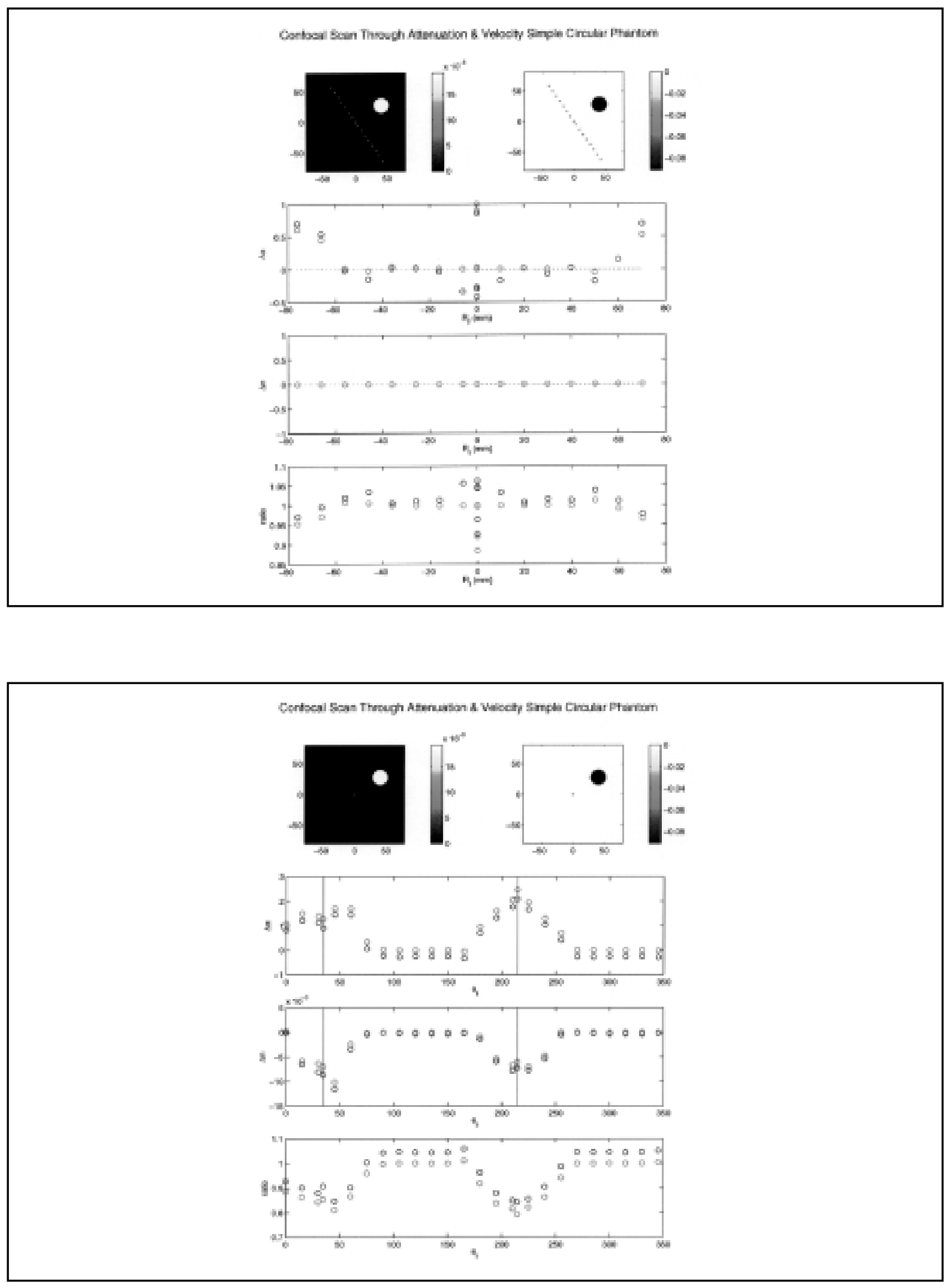


\title{
Filtered Backprojection for Low Contrast Medical Ultrasound Tomography Using Matlab
}

\author{
CASIS Workshop
}

November 11-12, 1999

\author{
David Scott \\ Sean Lehman \\ Lee Haddad \\ David Chambers
}

\section{Image Reconstruction Protocol}

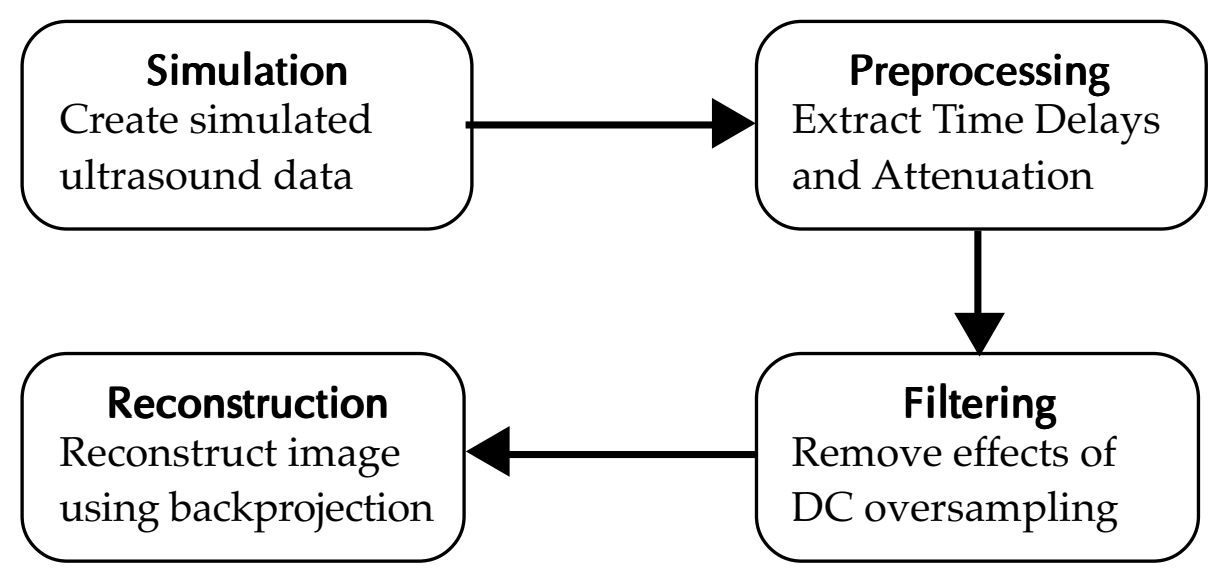




\section{A Simple Test Object}

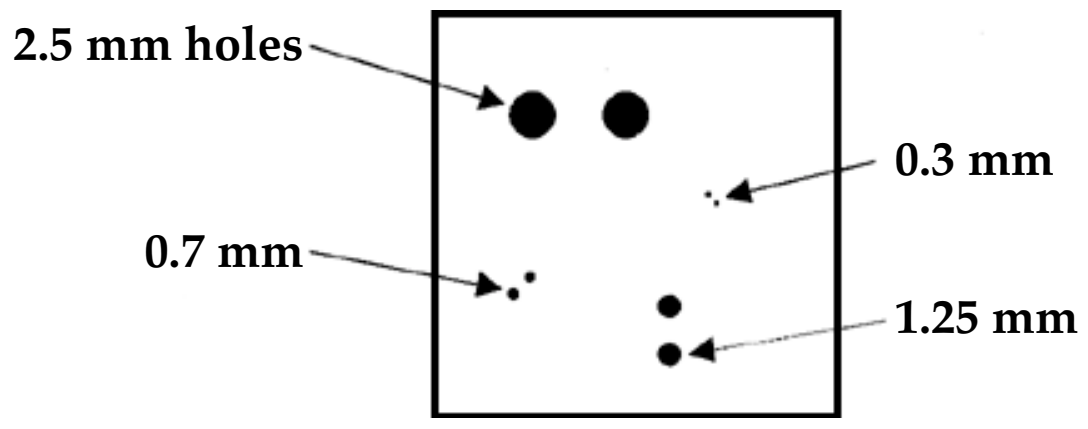

\section{Simulating Ultrasound Data}

Simulates 2D and 3D

ultrasound propagation

Model is accurate for reconstructing transmission and reflection modes images

Geometry is easily configurable

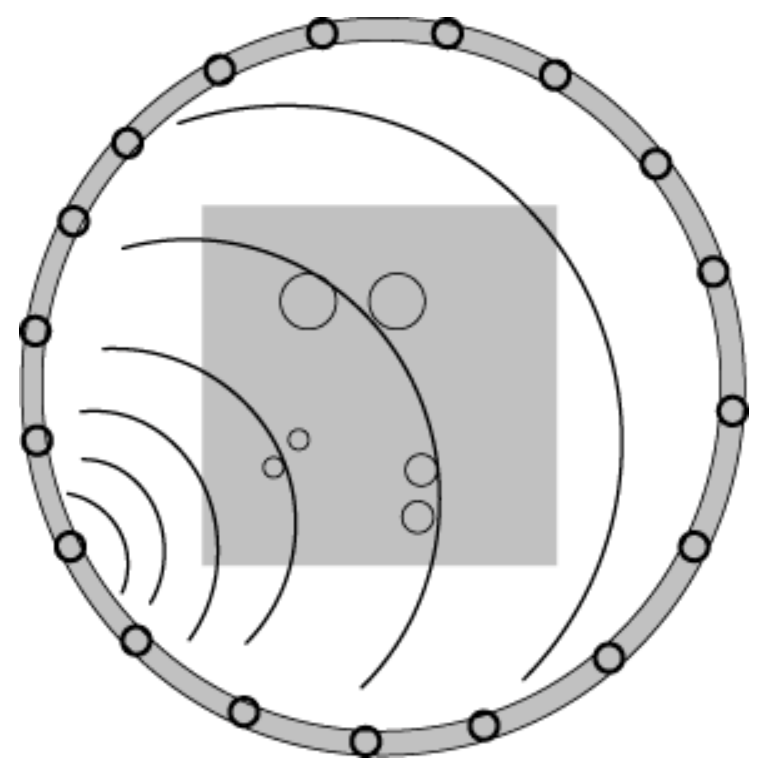




\section{Filtering the Projection Data}

The "ramp" filter reduces the effect of oversampling the lower frequencies.
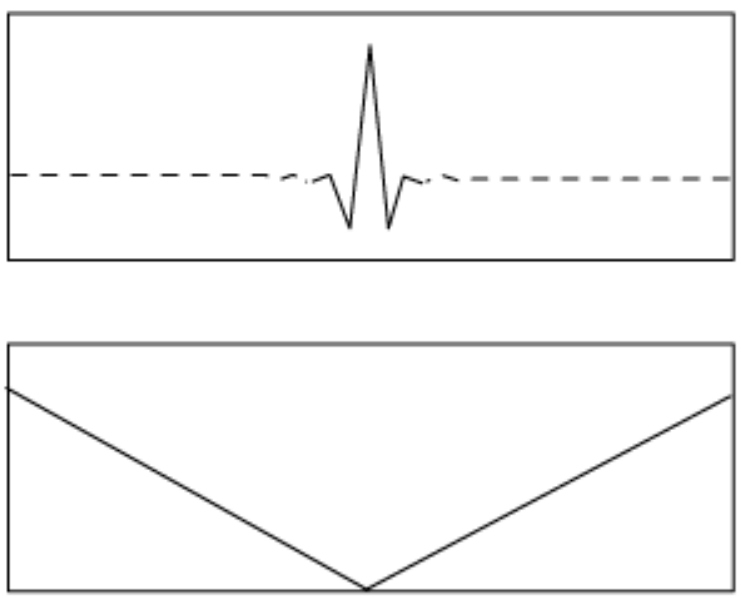

\section{Filtered Backprojection: The Fourier Slice Theorem}

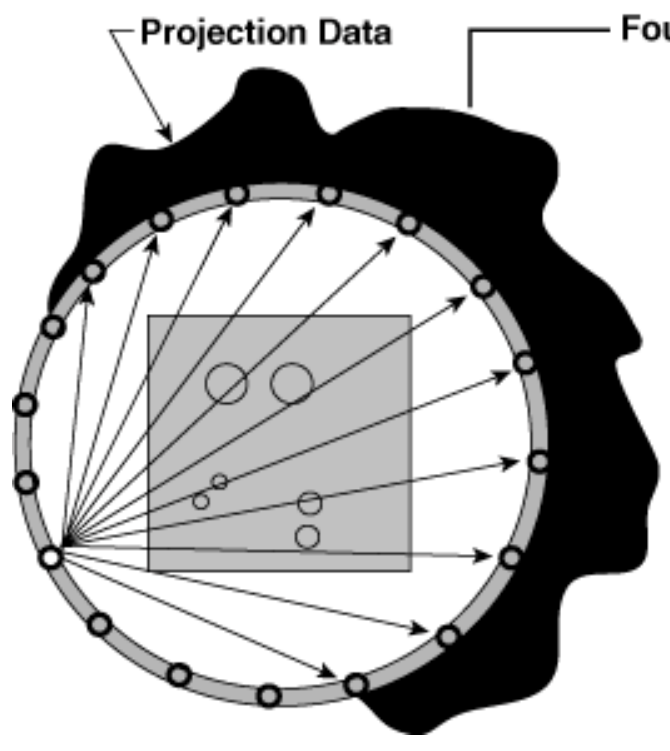

Fourier Transform

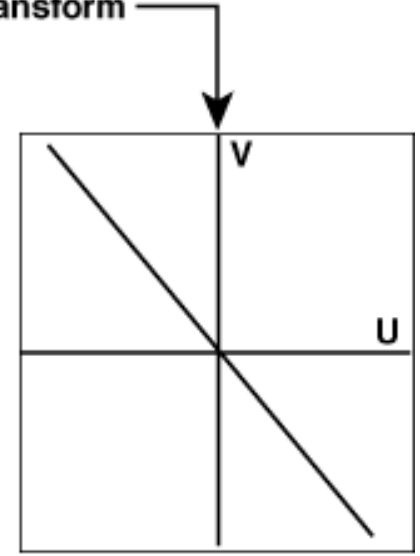


C O M P U T E D T O M O G R A P H Y

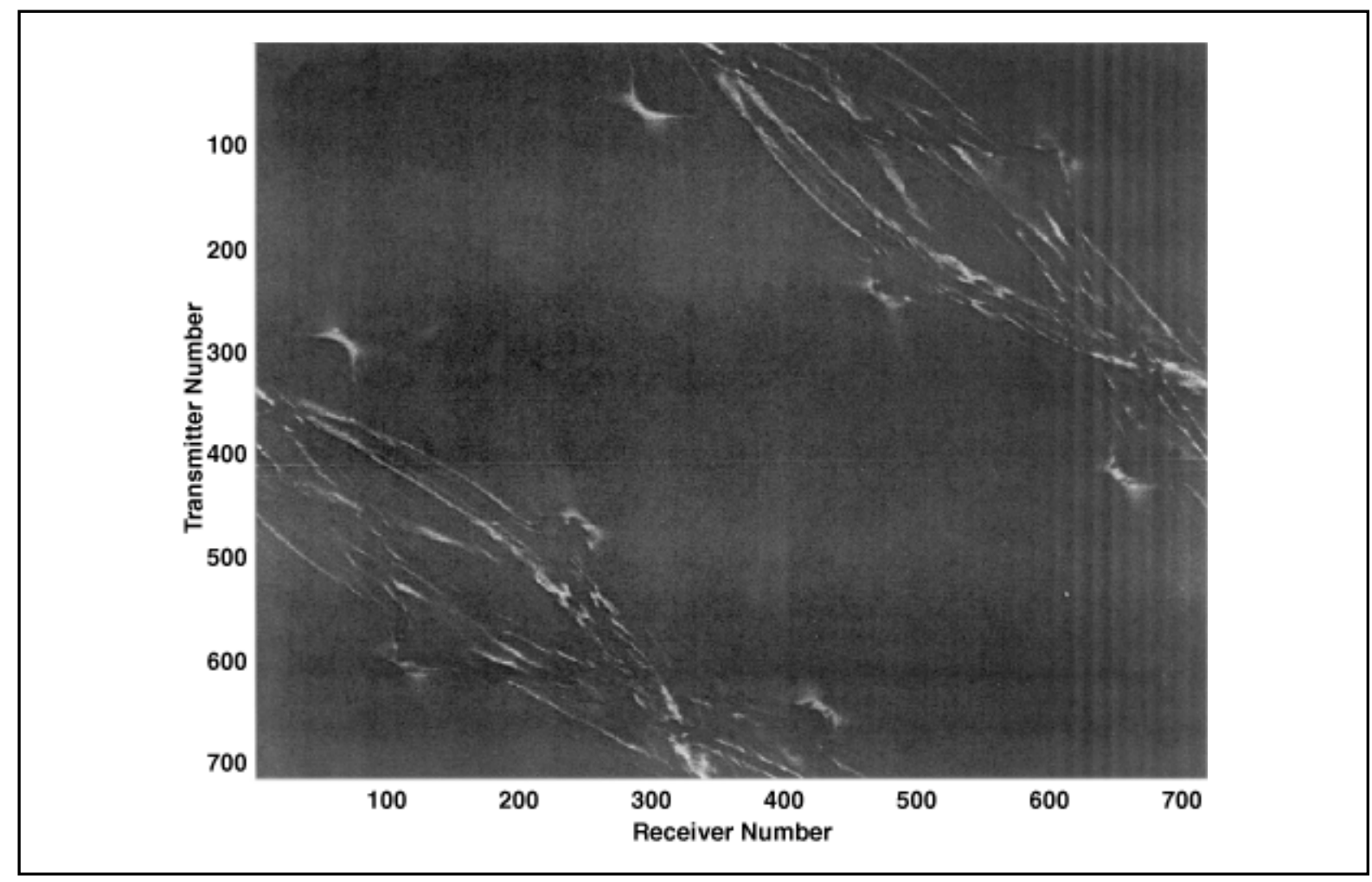

\section{Extracting the Time Delay and Attenuation}

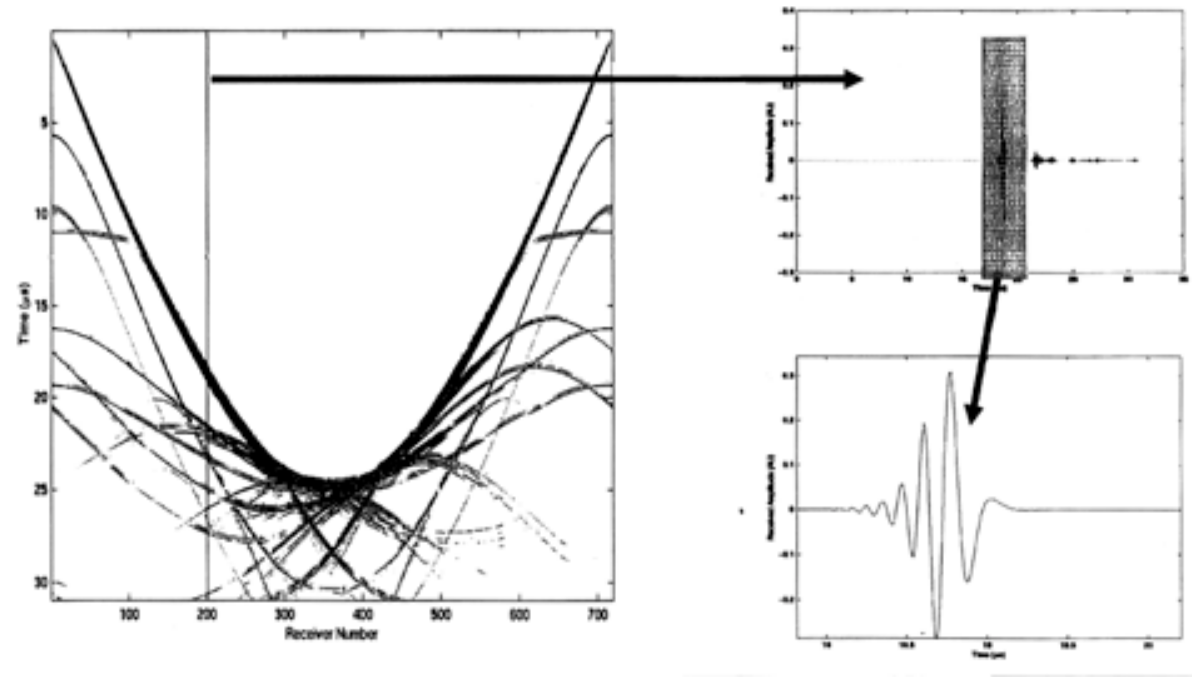


C O M P U T E D T O M O G R A P H Y

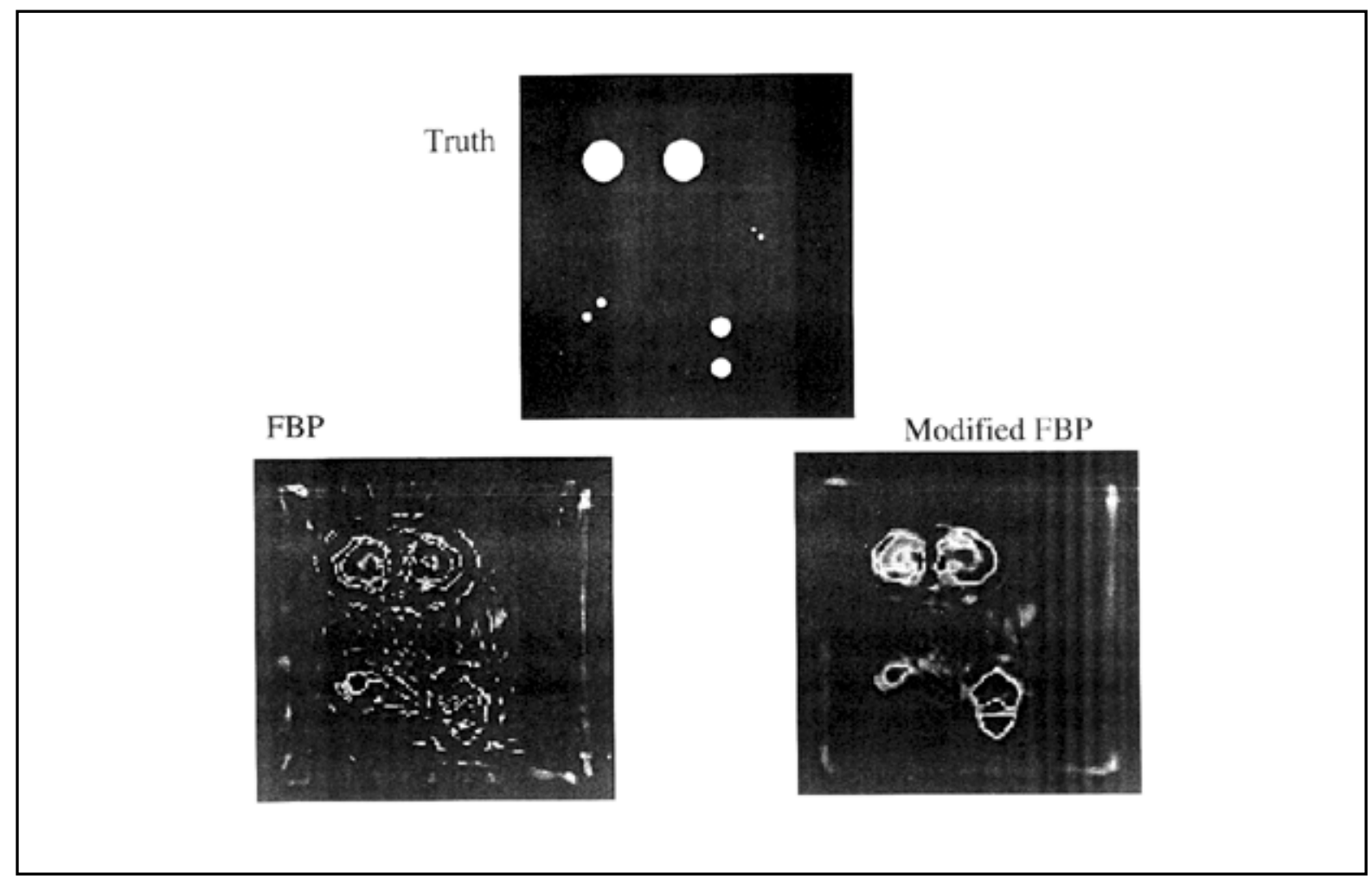




\title{
Optimal Beam Hardening Correction for X-Ray Tomography
}

\author{
Jesse Kolman
}

Images produced by x-ray tomography using a high-energy broadband source are distorted by beam hardening, which is the result of the fact that the attenuation of x-rays varies with wavelength.

If the object being imaged consists of a single material, the effects of beam hardening can be removed effectively.

However, for objects composed of multiple materials, the image reconstruction problem is inherently ill-posed and cannot be solved without a priori information.

We have developed an algorithm which is capable of reconstructing an image of an object containing multiple materials when a priori knowledge is available. The algorithm uses a constrained optimization algorithm to find the maximum likelihood estimate of the object.

In general, the algorithm can be expected to be successful when the a priori information reduces the ill-posed nature of the problem adequately. In the absence of such information, the algorithm returns a solution which may be suboptimal, since the inherent ambiguities cannot be resolved. In such cases, useful images can often still be obtained, at a high computational cost.

keywords: beam hardening, $x$-ray tomography, optimization Algorithms

\section{Optimal Beam Hardening Correction for X-Ray Tomography}

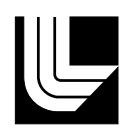

Jesse Kolman

November 11, 1999 


\section{High Energy X-Ray Tomography}

- High energy (short wavelength) $\mathrm{x}$-rays are needed to penetrate dense materials

- Sources of high energy $\mathrm{x}$-rays are typically broadband

- These conditions result in beam hardening

\section{Beam Hardening}

- X-rays with high energy are attenuated less than those with lower energy

- Transmitted x-rays have a higher relative concentration at high energies

- Attenuation is material dependent 


\section{Beam Hardening Model}

- The measurements are related to the object by

$$
\hat{y} i=\sum_{k} s_{k} \exp \left\{-\sum_{m} \alpha_{m k} A_{i} \underline{x}_{m}\right\}
$$

where

$\underline{\mathbf{x}}_{\mathrm{m}}=$ Sources of high energy $\mathbf{x}$-rays are typically broadband

$\underline{A} \underline{x}_{m}=$ These conditions result in beam hardening

$S_{k}=$ Sources of high energy $x$-rays are typically broadband

$\alpha_{\mathrm{mk}}=$ These conditions result in beam hardening

\section{Single Material Algorithm}

- If the object consists of a single material, the model becomes

$$
\hat{y} i=h\left(A_{i} \underline{x}\right)
$$

where

$$
h(x)=\sum_{k} S_{k} \exp \left\{-\alpha_{k} x\right\}
$$

- Inverting this pointwise nonlinearity results in a standard linear inverse problem 


\section{Optimization Problem}

- If the measurement noise is independent identically distributed Gaussian noise, then the maximum likelihood estimate of the object is the minimizer of

$$
f(\underline{x})=\frac{1}{2} \sum_{i}\left(\hat{y}_{i}-y_{i}\right)^{2}
$$

Subject to any known constraints

\section{Optimization Algorithm}

- We use a constrained conjugate gradients algorithm, which iteratively searched for a solution using the gradient

$$
\nabla \underline{x}_{l} f=A^{T} u_{l}
$$

where

$$
u_{l i}=(\underline{\hat{y}}-\underline{y}) \sum_{k} S_{k} \alpha_{m k} \exp \left\{-\sum_{m} \alpha_{m k} A_{i} \underline{x}_{m}\right\}
$$

- The algorithm dynamically enforces various constraints including nonnegativity 


\section{Difficulties}

- Typically, the number of object pixels is approximately the same as the number of measurements

- With one variable per material per pixel, the problem is ill-posed for multiple materials

- Many objects can result in the same set of measurements

\section{A Priori Knowledge}

- To make the problem tractable, inherent ambiguities must be reduced using a priori information

- Prior knowledge must be accurate and possible to enforce as constraints on the variables 


\section{Region of Support Constraints}

- Certain materials may be known to exist only in certain parts of the image

- Disallowing solutions which have materials outside their regions of support effectively reduces the number of variables

- Good results can be obtained even with overlapping regions, as long as the number of variable is no more than the number of measurements

\section{Initial Estimate}

- We can start the algorithm with an initial guess at the object based on a blueprint

- Deviations or defects in the object can be accurately imaged if the true object is close to the initial estimate, even with multiple materials 
C O M P U T E D T O M O G R A P H Y

\section{Conclusions}

- If model parameters are accurately known and a priori information can sufficiently reduce ill-posedness, the problem can be accurately solved with an optimization algorithm

- Computational cost is high by manageable

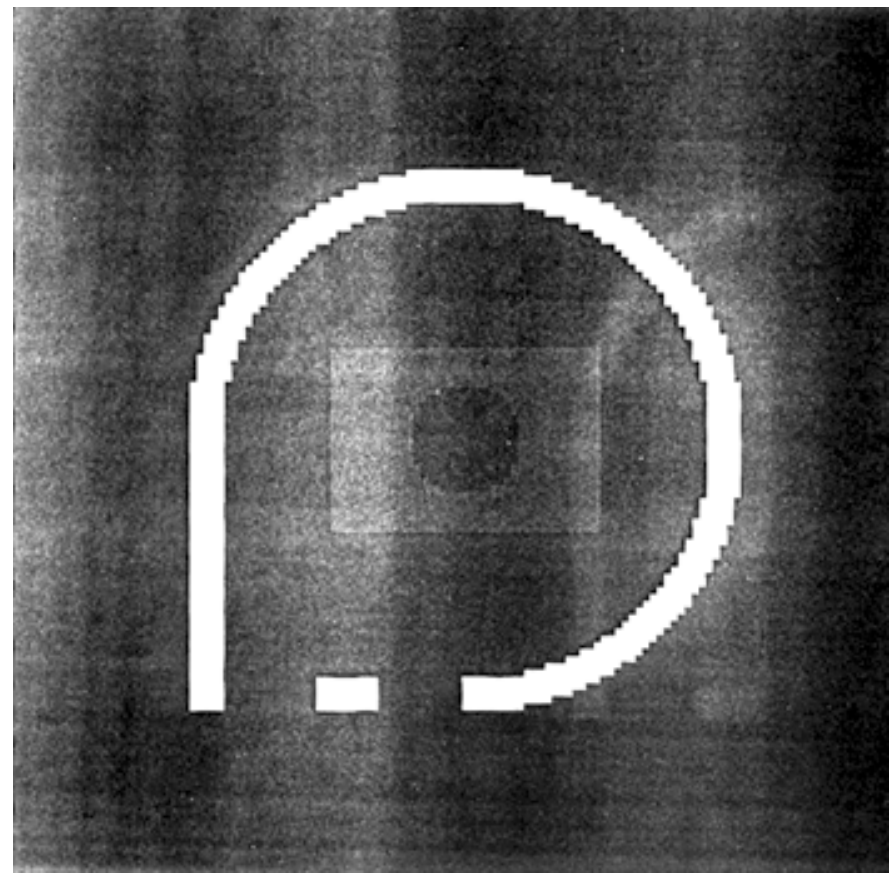




$$
\text { C O M P U T E D T O M O G R A P H Y }
$$
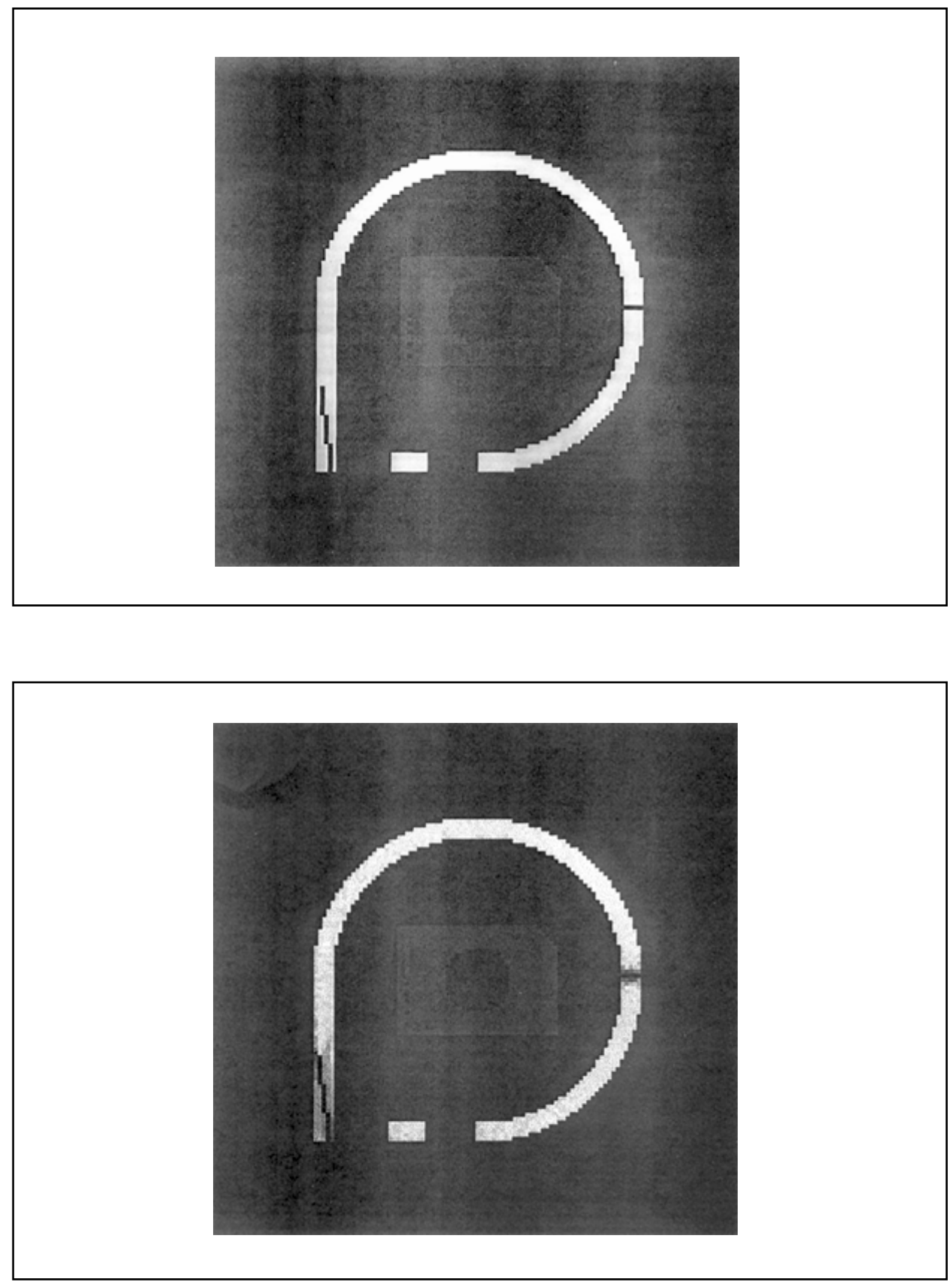


\title{
Performance Evaluation of Amorphous-Silicon Flat Panel Array for X-Ray Digital Radiography and Computed Tomography
}

(UCRL-VG-136259)

\author{
K. W. Dolan, J. J. Haskins, C. M. Logan , R. D. Rikard, and D. J. Schneberk
}

\section{SUMMARY}

Lawrence Livermore National Laboratory (LLNL) has evaluated amorphous-silicon flat panel arrays for use as imaging elements in x-ray digital radiography and computed tomography systems. Digital radiography systems are being developed for applications where digital image format and fast image acquisition provide an advantage over film, when image quality and dynamic range requirements can be satisfied. Computed tomography systems based on the flat panel technologies are being evaluated as a means of improving the speed of image acquisition as well as image quality, particularly for large fields-ofview (greater that a 5 or 6 inches on a side).

The flat panel amorphous-silicon (a-Si) array used in these studies provided a sensitive area of $195 \mathrm{~mm} \times 244 \mathrm{~mm}$ (7.7 in x $9.6 \mathrm{in}$ ) with 2.95 million pixels, where pixel dimensions are $127 \mu \mathrm{m} \times 127 \mu \mathrm{m}$ (0.005 in $\times 0.005 \mathrm{in})$. The array incorporates charge sensitive amplifiers and 12-bit A/D converters providing a dynamic range, as determined here, of approximately 3400:1. The a-Si array converts light images into electrical charge, pixel by pixel. The light images are produced by $x$-ray interactions in a phosphor screen placed in intimate contact with the a-Si array. Evaluations were made of the resolution and efficiency of different phosphor screens, filter and foil set combinations. Spatial resolution and contrast sensitivity were determined as a function of x-ray source energy from $70 \mathrm{kV}$ to 9 MV. Dynamic range, image acquisition speed, image reproducibility, potential for latent image formation, dark current, and corrective measures for source instability were also studied. Data acquisition and image normalization software were developed for both digital radiography and computed tomography. Application examples are discussed. 


\section{O M P U T E D T O M O G R A P H Y}

UCPL-WG: 136259

\section{Performance Applications of Amorphous-Silicon Flat Panel Imager}

prepared by

K. W. Dolan, J. J Haskins, C. M. Logan, R. D. Rikard and D. J. Schneberk presented by Ken Dolan

Nondestructive and Materials Evaluation Section

Lawrence Livermore National Laboratory

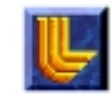

presented to CASIS Workshop

Livermore, CA

November 11-12, 1999

Work pertormed under the susposes of he U. S. Oeswrtment of Energy by lamrence

The a-Si flat panel array provides a new capability for radioscopy and computed tomography

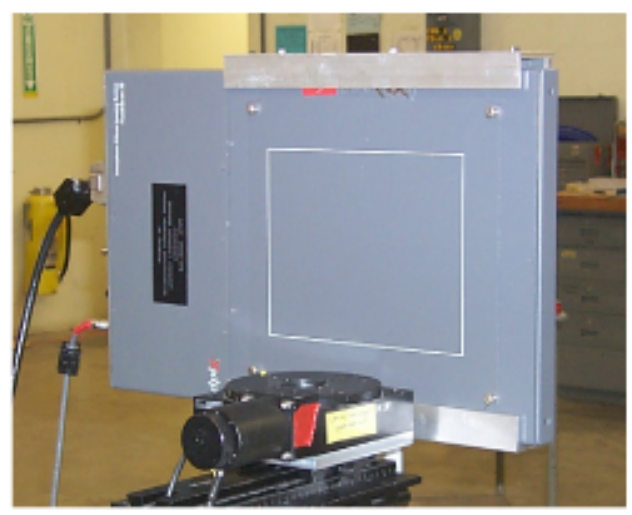

Flat panel a- Si array configured for radioscopy and computed tomography with rotation/ translation staging
Features

- Image sensor type

a-Si with conversion screen

- Image area

$195 \times 244 \mathrm{~mm}(8 \times 10 \mathrm{in})$

$282 \times 406 \mathrm{~mm}(11 \times 16$ in)

- Pixel format

$1536 \times 1920(2.9 \mathrm{M}$ pixels $)$

$2232 \times 3200(7.1 \mathrm{M}$ pixels $)$

- Pixel size: $\quad 127 \mu \mathrm{m}$

- Fill factor: $\quad 57 \%$

- Dynamic range: $>2000: 1$

- MTF: $>35 \%$ at $2 \mathrm{lp} / \mathrm{mm}$

- Image transfer time:

$<2 \sec$ (2.9 M pixels)

$<5 \sec (7.1 \mathrm{M}$ pixels) 
a-Si flat panel satisfies radioscopy and computed tomography needs for large area imaging

Advantages

- Flat profile for film replacement (1.8 in depth)

- Provides large area imaging ( $11 \times 16$ in) $\times 4$ panels

- Performs well at all energies tested (120 kV to $9 \mathrm{MV}$ )

- Electronics can be shielded outside image area

- Can choose phosphor screen to match x-ray flux

Limitations

- Dynamic Range $<12$ bits

- Fixed pixel size (127 $\mu \mathrm{m})$

- Readout electronics susceptible to radiation damage

- Each bank in array has different gain (128 rows/bank)

- Each pixel needs to be calibrated for each use

- Frame transfer time (2 - $5 \mathrm{sec})^{*}$

- Smaler array (1796238 mm) providas real-time (25-30 framestsec) at 2x2 binned

\section{Dark field and light field image corrections are needed to obtain calibrated image}

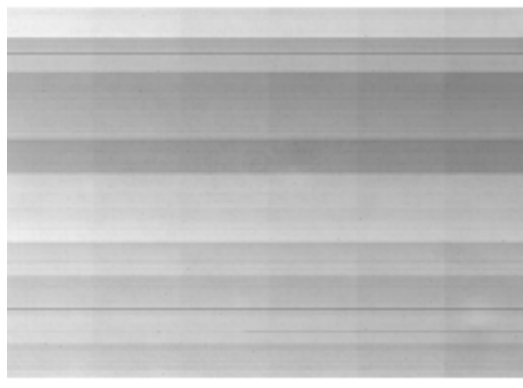

Dark field image at no $x$-ray fluence

Dark Field Image

Pixels start at maximum value $(3700-4000)$

128 pixel row elements per bank

Gain or offset differences between banks

Small additive bias across panel

A few bad pixels are also part of the image

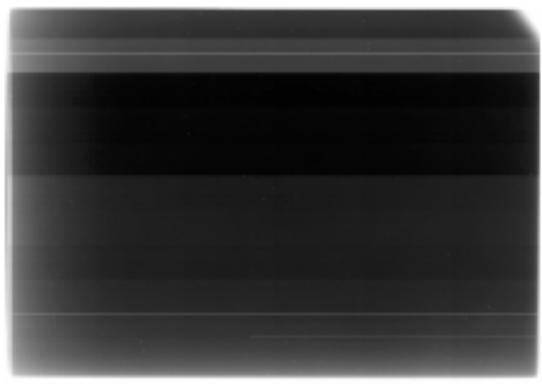

Light field image at maximum $x$-ray fluence

Light Field Image

Panel responds to light by counting down

Maximum usable pixel value is approx. 500

Dynamic range is variable between banks

Clipping in corners of this image is caused by collimation 


\section{Gain response is bimodal linear and varies slightly by pixel and bank}

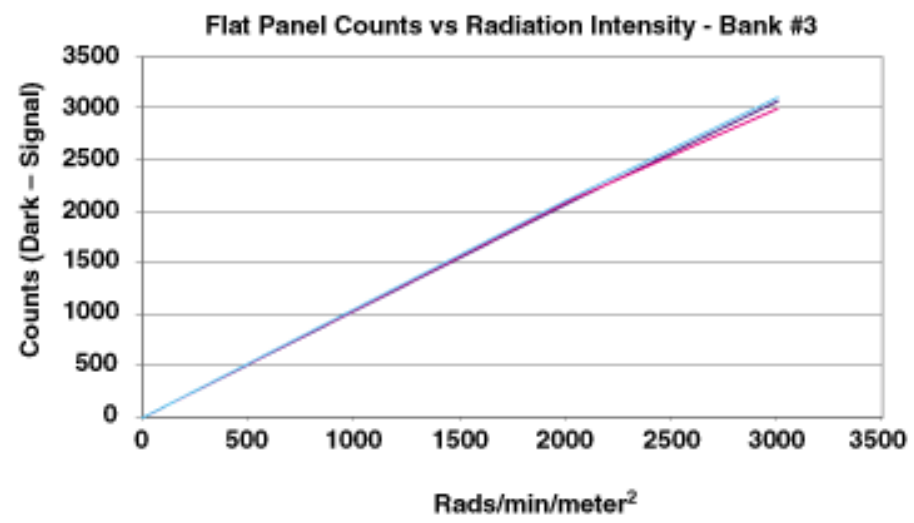

Plot shows pixel digital readout for 3 pixels in bank \# 3 as a function of $x$-ray exposure.

- Small but significant gain difference between pixels.

- Bimodal linear gain with change in slope at approx. $2000-2200$ counts

LLNL processing protocol for digital radiography and computed tomography

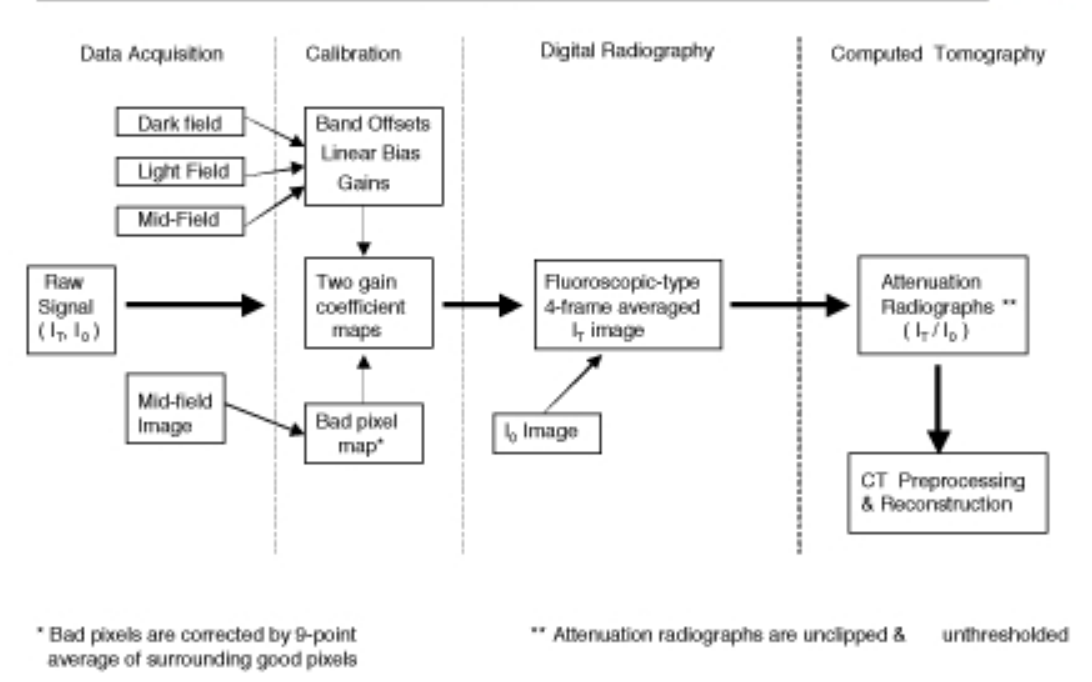


C O M P U TE D T O MO G R A P H Y

LLNL data analysis protocol has produced valid computed tomography results

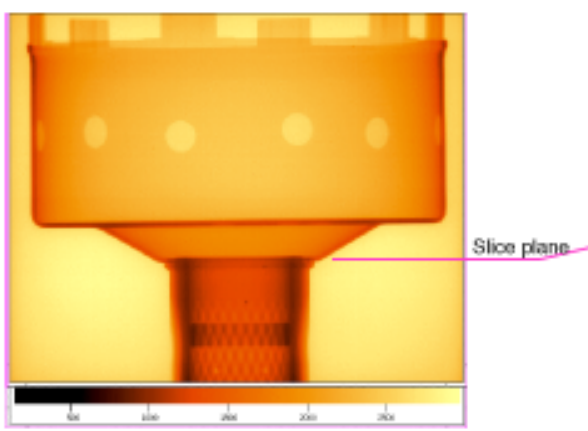

Digital radiograph of prototype clutch retainer

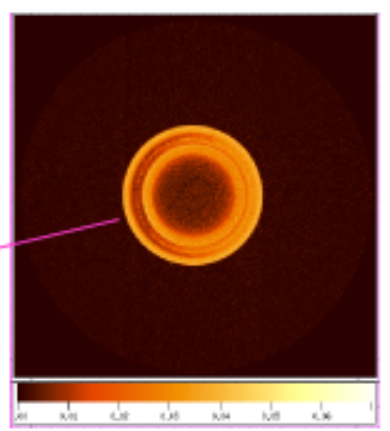

Computed tomography sice at laser weld joint

\section{An array of phosphor screens and scintillators were tested for signal conversion and resolution}

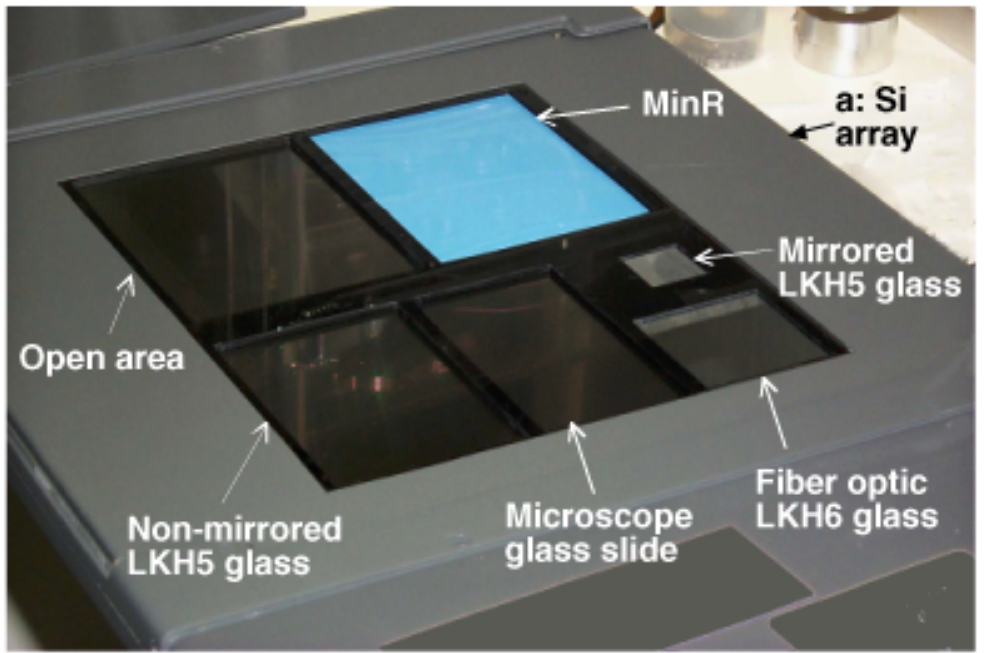

Mosaic arrangement of screens and scintillators in a: Si array imaging fixture 


\section{Phosphor screens with a: Si outperformed} scintillators and metal foil/screen combinations

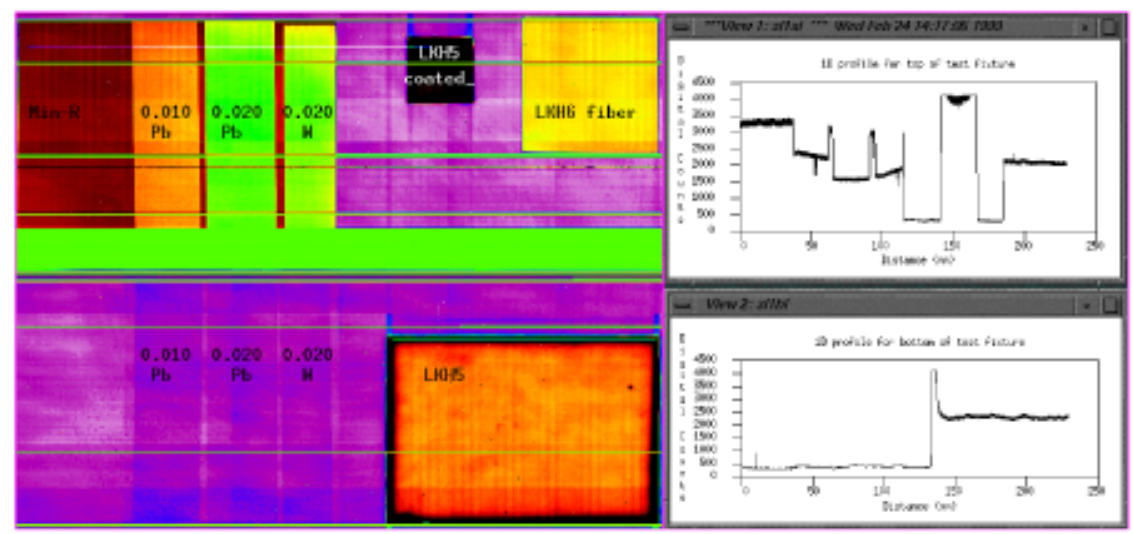

Best performance was obtained with Kodak MinR (Lanex Fine) phosphor screen

Resolution and MTF were determined from digital images of line pair gauges and block edges
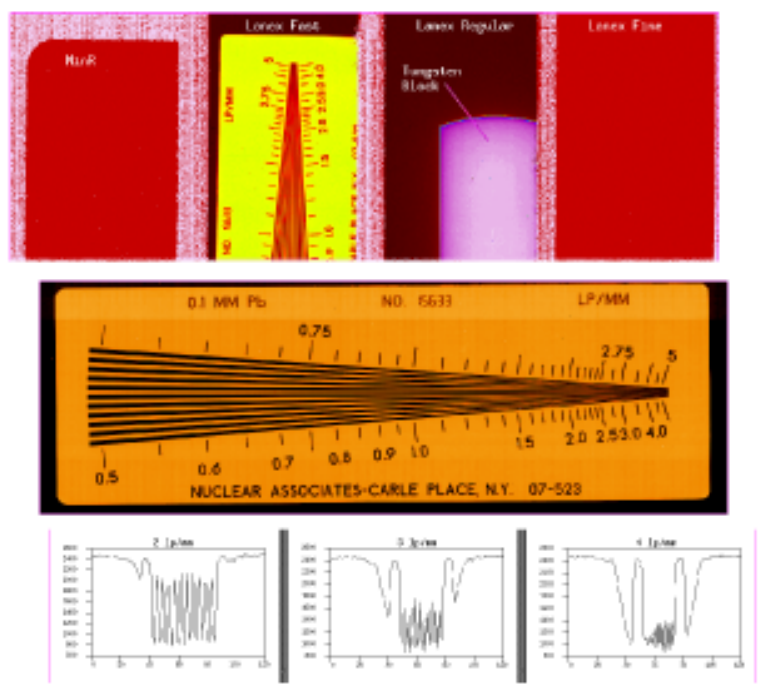
C O M P U T E D T O M

Modulation transfer function (MTF) was determined by the edge spread method

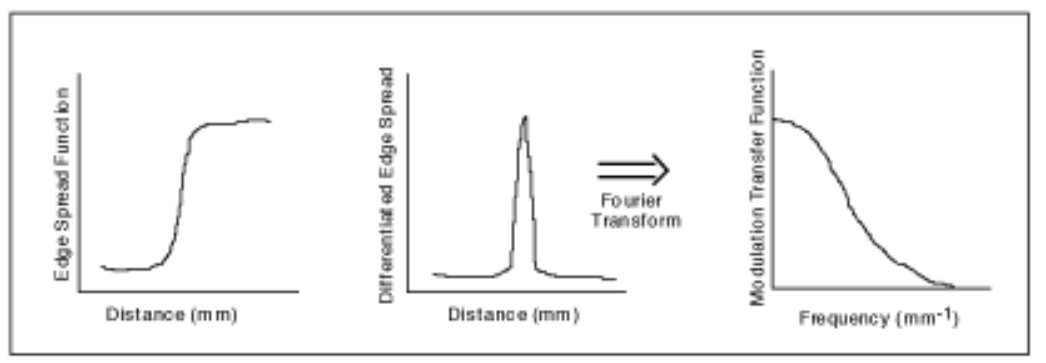

Schematic of edge spread method for determining MTF

MTF provides contrast as a function of feature size

MTF was determined with MinR screen for filtered and unfiltered x-rays at 120,250 and $450 \mathrm{kVp}$

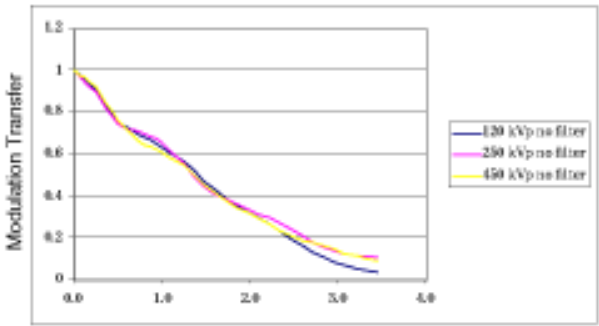

MTF is greater than $5 \%$ at $3.5 \mathrm{lp} / \mathrm{mm}$

For unfiltered spectra

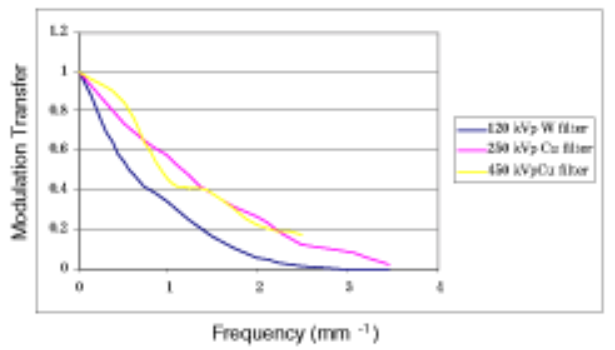

MTF is greater than $5 \%$ at $3.0 \mathrm{lp} / \mathrm{mm}$

$120 \mathrm{kV}$ and $250 \mathrm{kV}$, greater than $5 \%$

at $20 \mathrm{lp} / \mathrm{mm}$ for filtered spectra 
Modulation transfer functions were determined for 4 different screens at 3 different energies
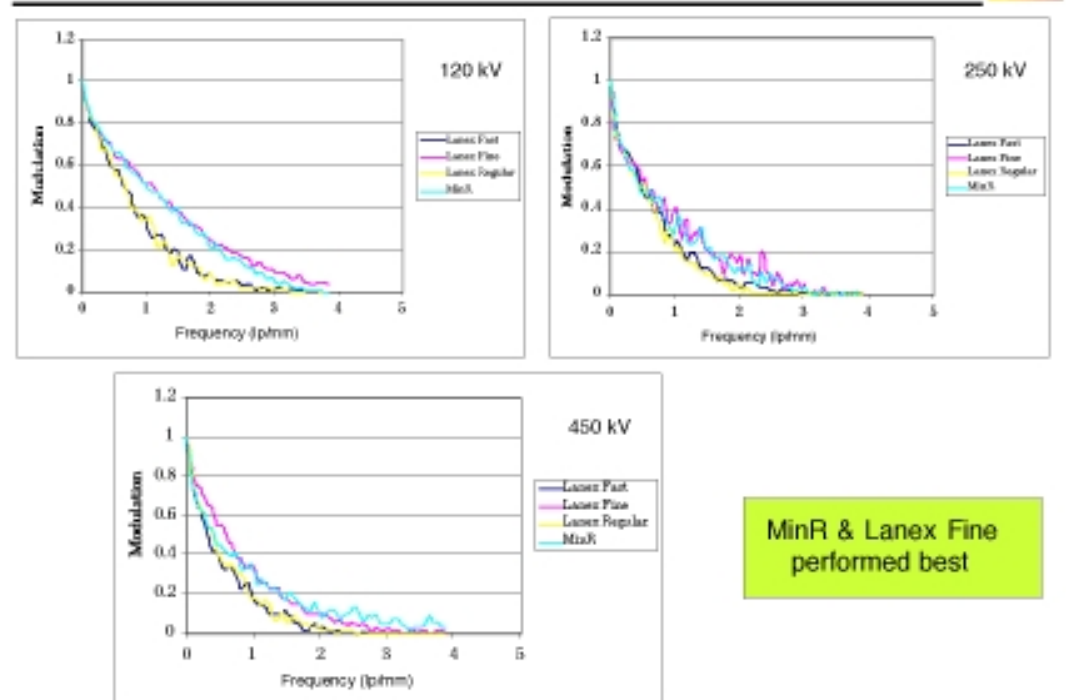

MinR \& Lanex Fine performed best

Exposure requirements $(\mathrm{ER})^{*}$ were determined for x-ray source energies 70 to $450 \mathrm{kVp}$

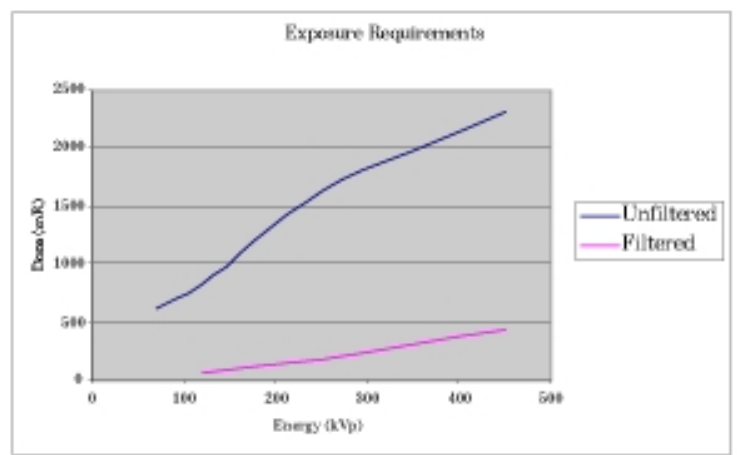

" ER is exposure that produces near saturation (light field) image 
a-Si computed tomography of steel bridge pin 3 inch diameter at $9 \mathrm{mV}$
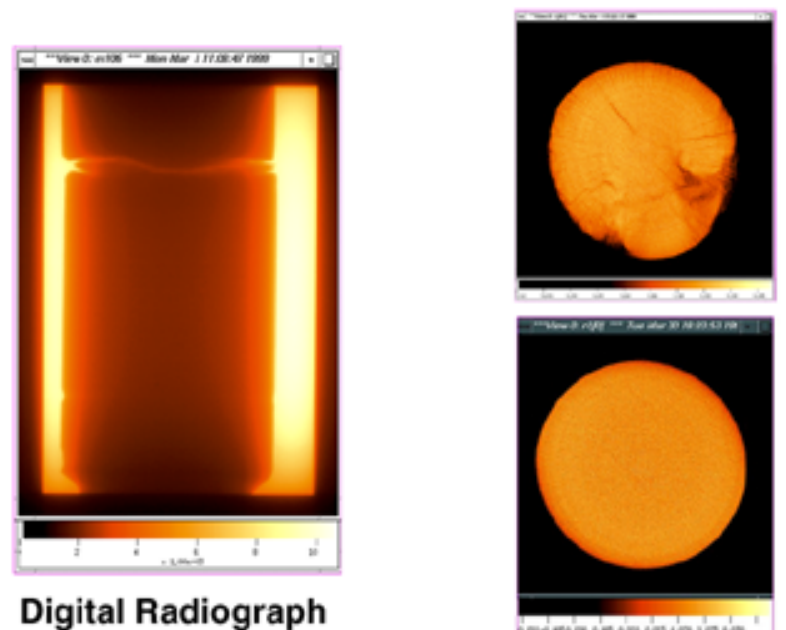

Digital Radiograph

Tomograms

a-Si computed tomography of protoype clutch retainer gear at $4 \mathrm{mV}$

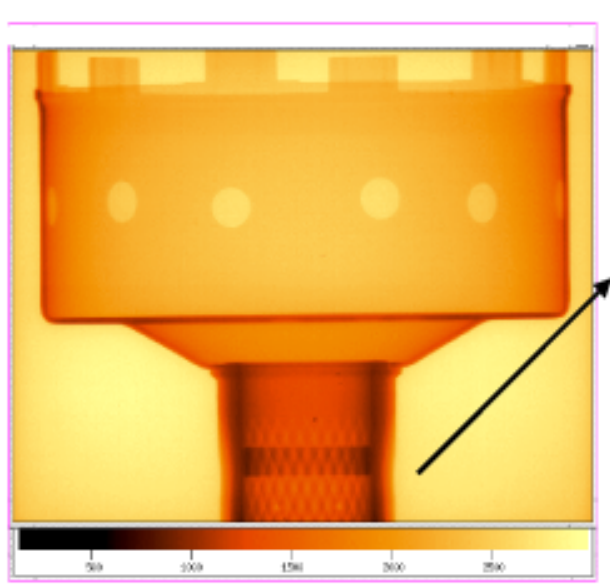

Digital Radiograph

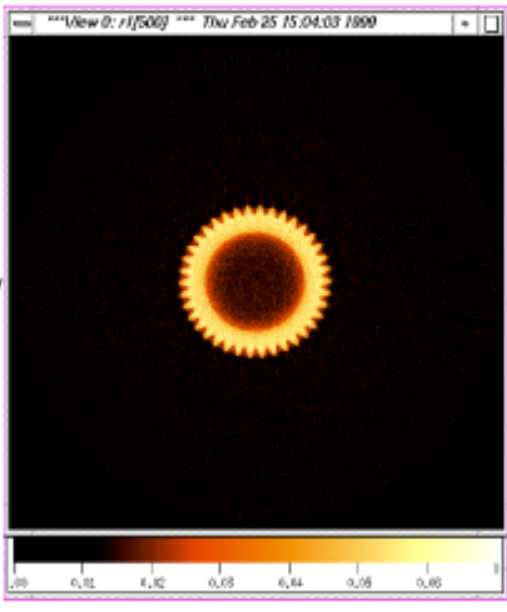

Tomogram 
C O M P U T E D T O M O G R A P H Y

Conclusions

A-Si flat panel imager provides a practical new imaging method for radioscopy and computed tomography , and opens new possibilities for fast digital image acquisition and quantitative analysis. 
C O M 
Astronomical

Imaging 


\section{Speckle Imaging of Saturn's Moon Titan}

Seran Gibbard, Bruce Macintosh, Claire Max, Donald Gavel: LLNL Imke de Pater, Henry Roe: UC Berkeley

Andrea Ghez: UCLA

Christopher McKay: NASA Ames

Eliot Young: Southwest Research Institute

\section{OUTLINE}

1. Speckle Imaging

2. Titan background

3. Our Titan observations

4. Modelling Titan's surface and atmosphere

5. Titan's surface

6. Conclusions 


\section{STEPS IN SPECKLE PROCESSING}

- Take short-exposure images (shorter than time constant for turbulence)

- Remove bias, sky, flat-field effects on each frame

- Combine image Fourier transforms to get object's power spectrum

- Then use bispectrum technique (Lohmann et al. 1983) to estimate phase

- Then convolve with power spectrum of unresolved point source, re-convolve with theoretical telescope PSF

- Resolution is limited by diffraction limit of telescope, not by 'seeing'

- Diffraction-limited resolution for 2 microns at Keck is 0.04 arcseconds

- Technique limited to bright objects since each short exposure must have sufficient signal/noise

\section{TITAN}

- Largest moon of Saturn - larger than Mercury

- Substantial nitrogen/methane atmosphere

- Photolysis of methane - atmosphere has complex organic chemistry

- Hydrocarbon haze may 'rain' out on surface

- Possible hydrocarbon 'seas'?

- Analogue for early Earth before life 


\section{SPECKLE IMAGING OF TITAN}

- Dates: Sept. 6 1996, Oct. 10-13 1997, July 29-Aug 1 1998, Oct. 7-14 1998 (UT)

- Telescope: 10-m W.M. Keck

- Instrument: Near Infrared Camera (NIRC) with image converter

- Plate scale: $\mathbf{0 . 0 2}$ arcseconds per pixel

- Resolution (diffraction limited) 0.04 " at $\mathrm{K}$

- Wavelengths: $\mathrm{K}^{\prime}(1.96-2.29 \mu \mathrm{m}, 4360-5100 / \mathrm{cm})$

$$
\text { H (1.49-1.82 } \mu \mathrm{m}, 5495-6710 / \mathrm{cm})
$$

- Data taken in sets of $\mathbf{1 0 0}$ frames, each frame $\mathbf{1 5 0}$ or $\mathbf{2 0 0}$ ms of integration

\section{ATMOSPHERIC MODEL}

- About $60 \%$ of light received from Titan at K' and $70 \%$ of light at $\mathrm{H}$ comes from atmosphere rather than surface

- By analyzing this light can constrain atmosphere

- Atmospheric model must reproduce:

- Observed limb brightening

- Observed asymmetry in N/S brightness (S hemisphere currently brighter)

- Overall brightness of planet

- Don't get negative surface albedo!

- Adjust haze parameters to minimize difference between model and limb of planet

- Model gives light received from the atmosphere; the remainder of the light comes from the surface 


\section{WHAT IS TITAN'S SURFACE MADE OF?}

- Bright regions may be ice, ice/rock mixture

- Dark regions could be:

- Crater basins filled with impact or volcanic material

- Low-lying regions into which organics are washed by methane rain in bright highlands

- Areas of infrared-dark ice

- Mixture of ice and organics?

- 'Seas' of liquid hydrocarbons

\section{CONCLUSIONS}

1. Speckle imaging gives excellent resolution for bright solar system objects

2. Limited to broadband filters

3. Atmosphere models useful to separate Titan's surface from atmosphere

4. Surface albedo map of Titan reveals bright and dark areaspossible solid organics or liquid hydrocarbons

5. Adaptive optics will allow narrowband imaging of Titan to better separate surface and atmosphere 


\title{
Astronomical Imaging Fourier Transform Spectroscopy: Technology and Techniques
}

\author{
Ron Wurtz
}

Our group is developing an astronomical ground-based facility-class visible/IR imaging Fourier transform spectrograph (IFTS). Beginning with a run at Kitt Peak in March 1999, this project has been demonstrating astronomical IFTS in the low flux regime with bench-top technology and techniques. Our demonstrations include multi-object spectroscopy, simultaneously obtained narrow-band imaging, time-induced noise removal, dispersed and Fourier transform hybrid spectroscopy, etc. We will discuss these techniques as well as the techniques of data calibration, reduction, and extraction.

\section{The LLNL Ground-Based Optical Imaging \\ Fourier Transform Spectrometer}

\author{
Presentation to \\ NGST Science \& Technology Exposition \\ Hyannis, MA
}

C. Physies [0.

Ron Wurtz*, Charles Bennett*, Jay Blxler, Dennis Carr, Kem Cook (PI)*, Cailin Nelson, and Edward Wishnow* IFIRS Team (includes "), James Graham (PI)

Lawrence Livermore National Laboratory

September 14, 1999 
The Next Generation Space Telescope (NGST) will probe "the Dark Zone" and answer key questions

How and when were the first stars and galaxies born?

- What is the mysterious dark matter?

- What is the shape of the universe?

A faster, cheaper, better mlssion -\$1 billion launch 2007

- 1-5 $\mu \mathrm{m}$

- Redshifted stollar output

- 8-m diffraction limited

- Faint objects

- High angular resolution

- $50 \mathrm{~K}$ operating temporature

- Low background

- L2 orbit

- Low background

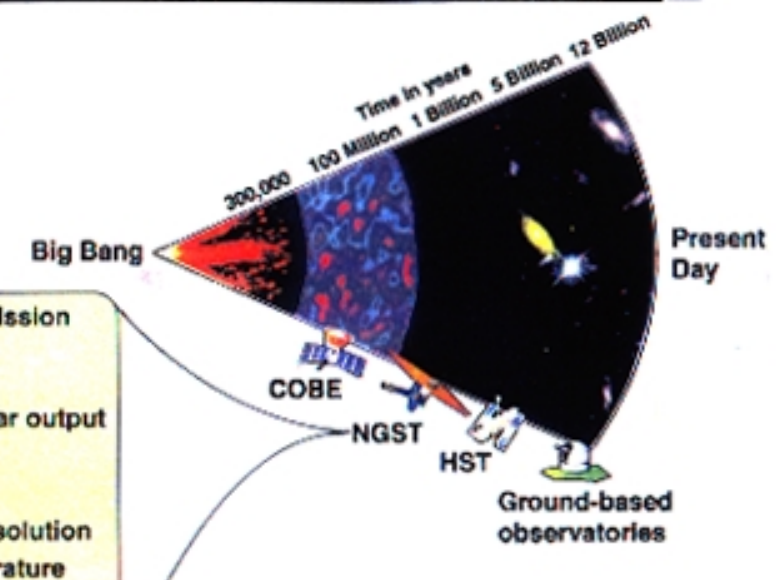

With unprecedented sensitivity comes unprecedented instrumental requirements

- In very deep images, virtually every pixel contains interesting sources

- Spectroscopy for every pixel is needed for scientific understanding

- High resolution is necessary both spatially and spectrally

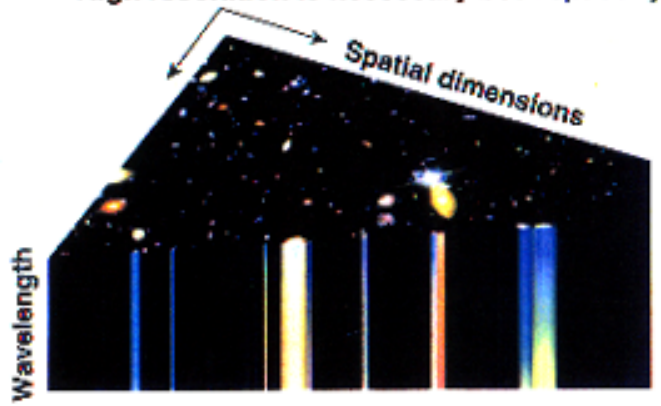

The "Hubble Deep Field" contains thousands of galaxies in a supposedly "empty" field

LLNL is an acknowledged world leader in infrared imaging spectroscopy: Developed HIRIS technology and hosted 3-D Imaging Conference 
A S T R O N O M I C A L I M A G I N G

An imaging FTS is ideal for NGST

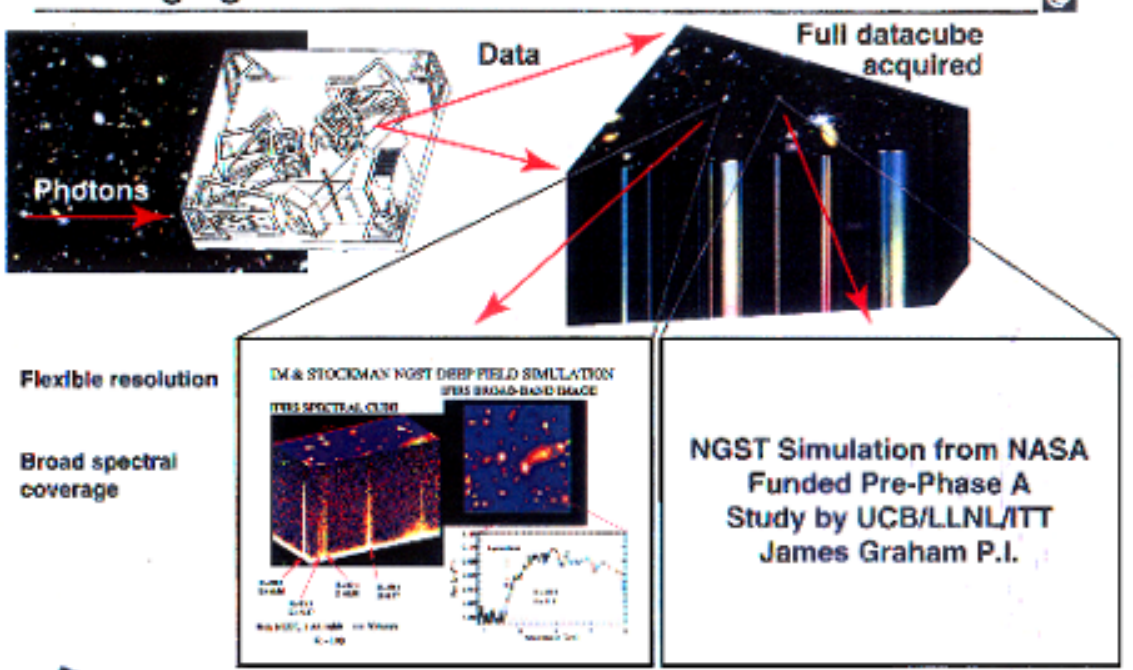

Simultaneous deep field imaging and spectroscopy for all pixels

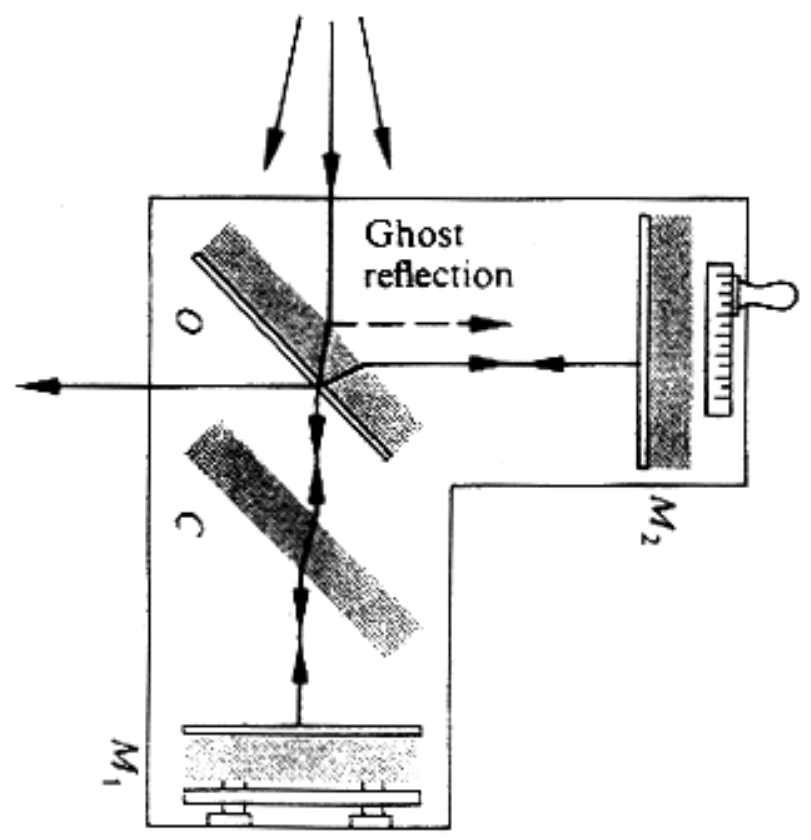




\section{Review of Imaging Fourier Transform Spectrometers}
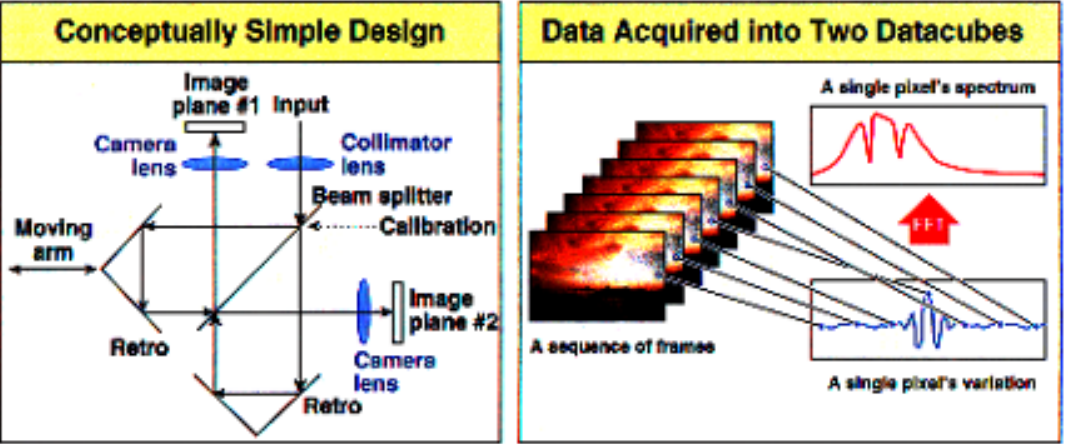

\section{NGST IFTS Concept Tests are Possible with} Ground-based Optical IFTS

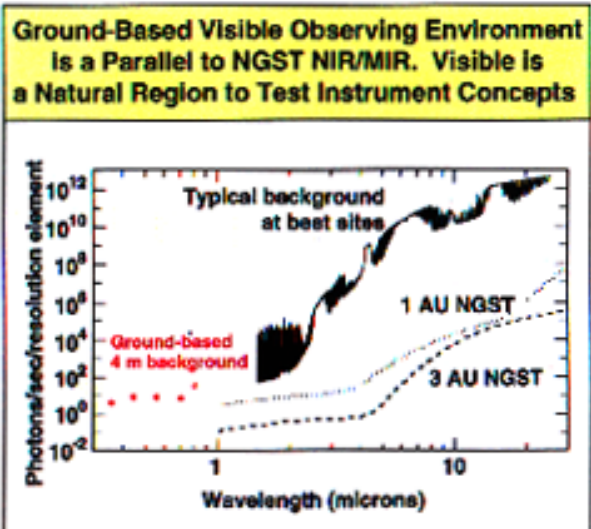

- Build Optical Astronomical Imaging Four-Port FTS and demonstrate:

- Visible FTS tolerances are met with existing technology

- A new regime: long dwell time step scan $(<1 \mathrm{~Hz})$

- Two output advantage

- Hybrid dispersed FTS

- Data reduction 
A S T R O N O M I C A L I M A G I N G

Tabletop IFTS with coaligned metrology system

닌

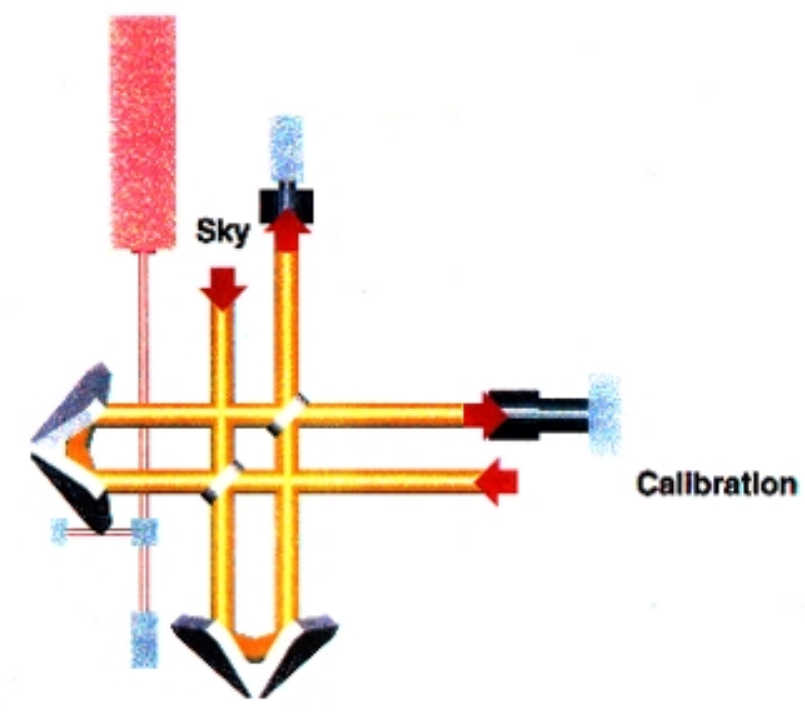

Porastrownocos

- -90

IFTS layout at NSO

16

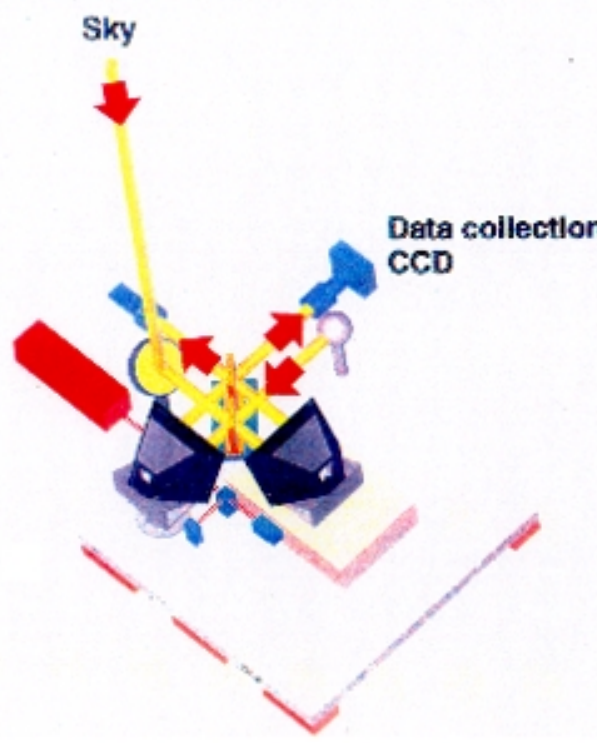


A S T R O NOMICAL I MAG I N G

\section{McMath-Pierce Main Spectrograph Port}

-1.5 Meter Primary

-Friendly to Visitor Instruments

-Horizontally Mounted Field De-rotator

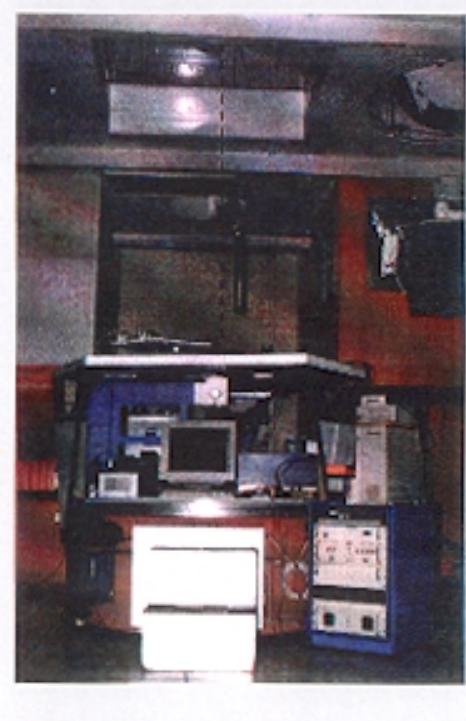

Insoming Vertical Bease

Rouating Optical Beoch (latecterometer goes bert)

Routing Spectroyraph

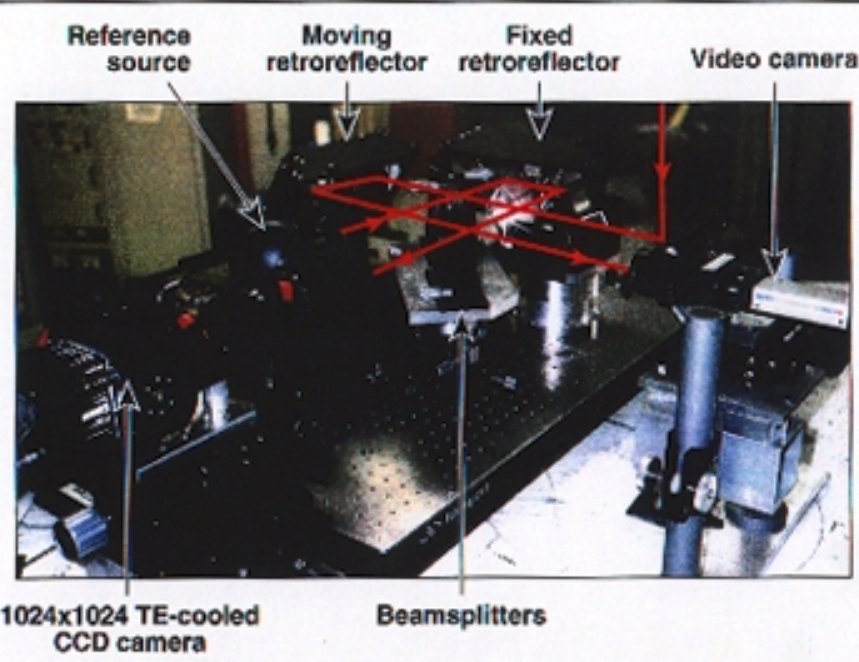

- First Generation: LLNL Instrument is Open Design - Second Generation: "Facility"-llike Instrument 
A S T R O NOM I C A L I MA G I N G

Kitt Peak: Southern edge of M4 (Single Output Port)
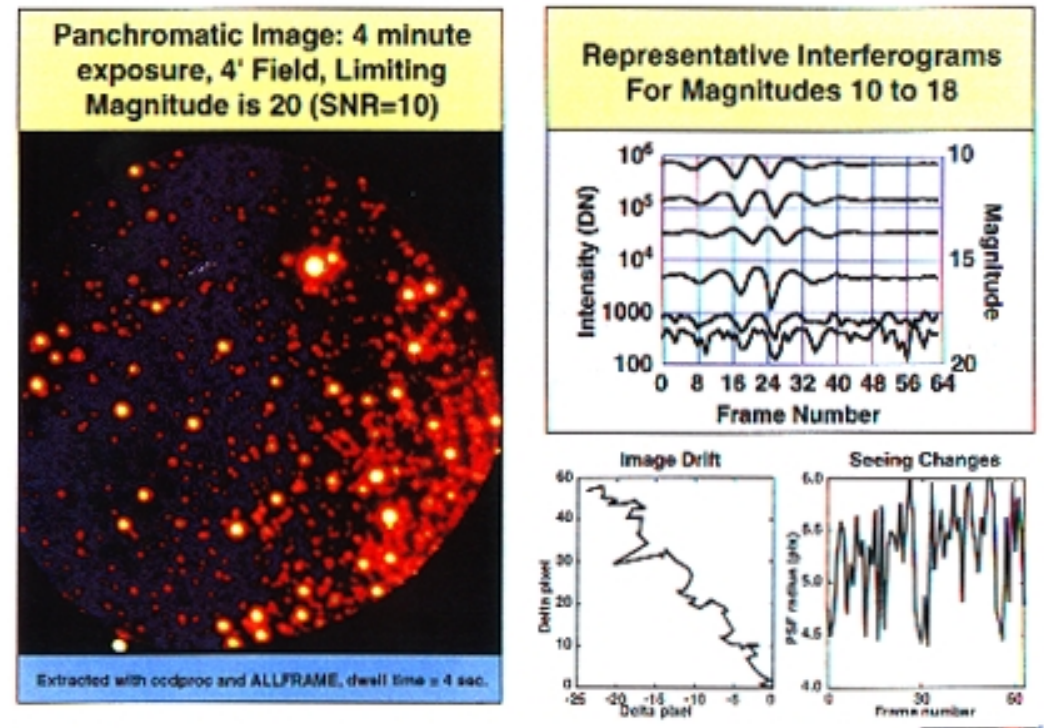

\section{Polaris: Demonstration of advantage of two output ports}

Kitt Peak data were obtained with one science output port. Adding a second sclence output allows for common mode rejection reduces noise from internal and extemal time variations in source and intervening absorbers.

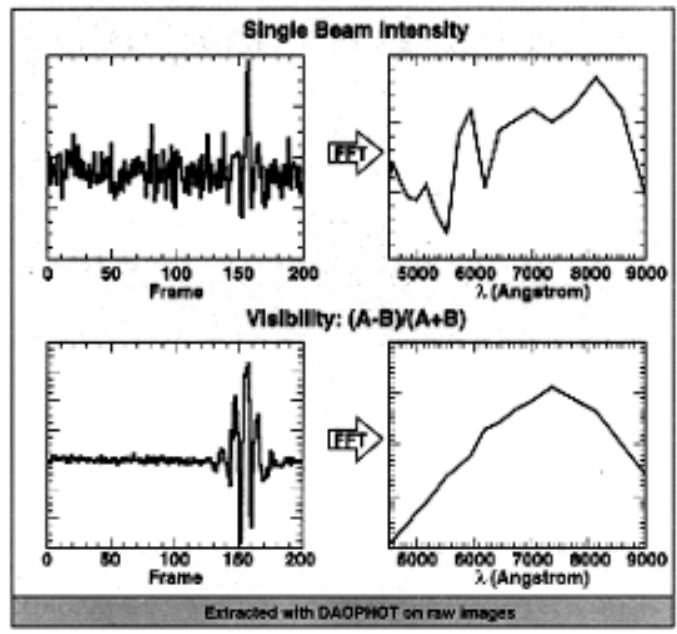



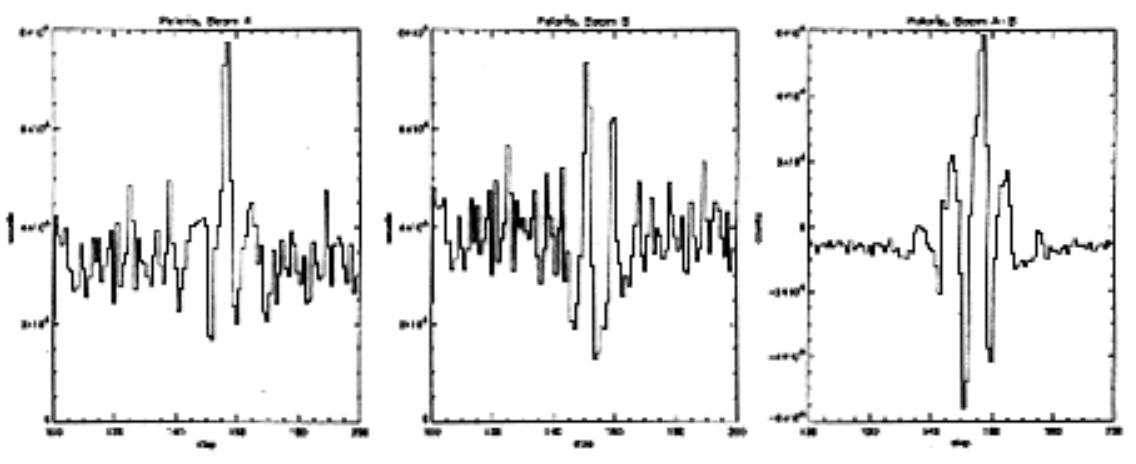

Kitt Peak: Orion Nebula (Single Output Port)

Extended region with widely spaced emission lines
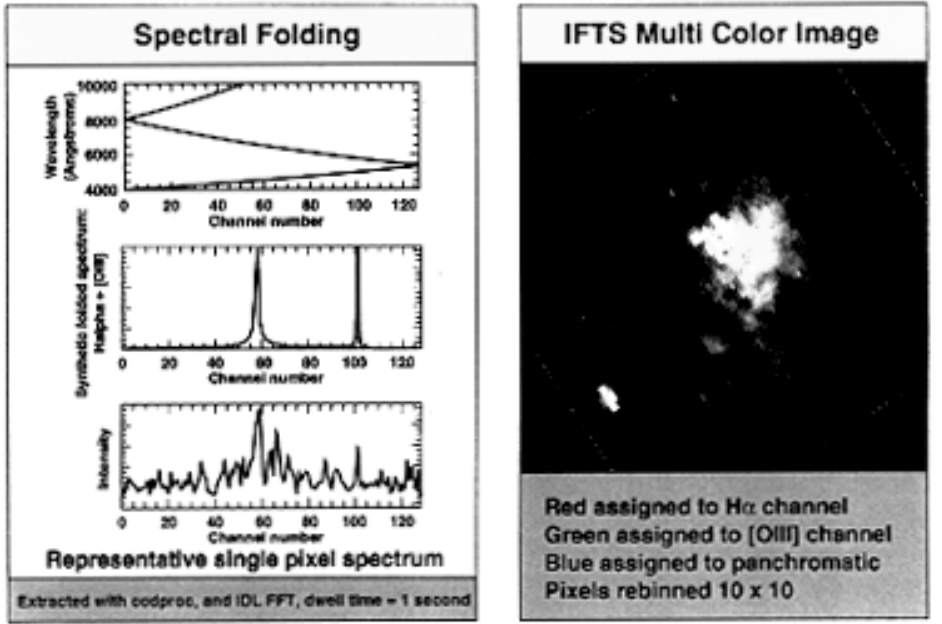

Green assigned to [Olin] channel

Blue assigned to panchromatic

Pixels rebinned $10 \times 10$ 
Polaris: Hybrid FTS and dispersing spectrometer (single output port)

Inserting a Dispersing Element (prlsm) Just before the camera makes a Hybrid Dlspersed FTS

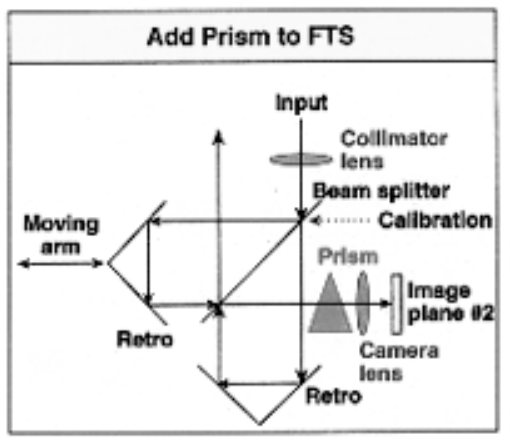

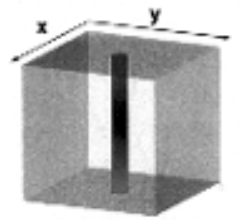

Undispersed FTS

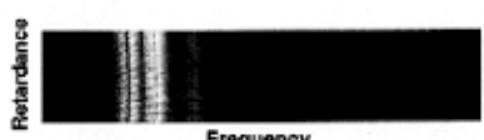

Frequency

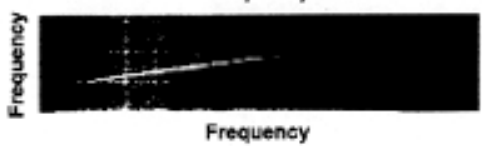

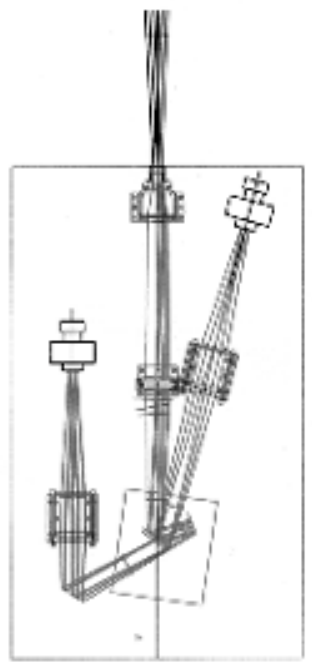

- Benchtop vislble IFTS has probed NGST regime:

- Shorter wavelengths (tight tolerances)

- Long integration times (step scan)

- Technology readiness

- Data reduction technique

- Varying spectral resolution

- Spectral folding

- Common mode rejection

- Hybrid FTS plus dlsperser

- Next:

- Vlsible Astronomical IFTS using technology developed specifically for NGST

- Fielded at 4-10m class telescope

- Fall 1999: ARC $3.5 \mathrm{~m}$

- 2000: Gemini/Keck? 
A S T R O N O M I C A L I M A G I N G

"First Light" Science Results from the Telescope Adaptive Optics System

Claire Max

Institute of Geophysics and Planetary Physics and University Relations Program

LLNL

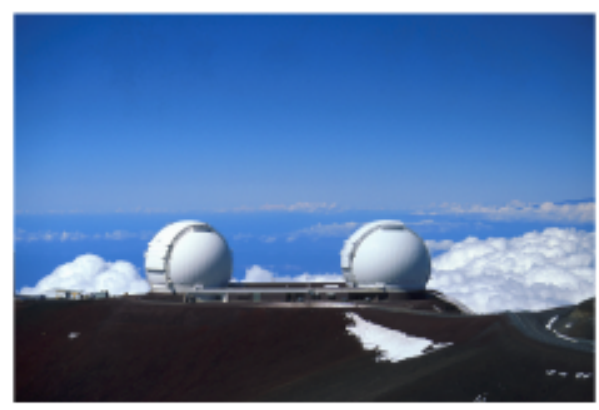

Adaptive optics system has been a joint project between Keck and LLNL staff

- Keck people:

- P. Wizinowich , S. Acton, O. Lai, C. Shelton, P. Stomski, ...

- LLNL people (laser program, engineering dept, IGPP):

- C. Max (PI), J. Brase, D. Gavel, B. Macintosh, S. Olivier, J. An, H. Friedman, G. Erbert, K. Waltjen, K. Avicola , K. Watson, ... 
A S T R O N O M I C A L I M A G I N G

\section{The Keck Telescope}

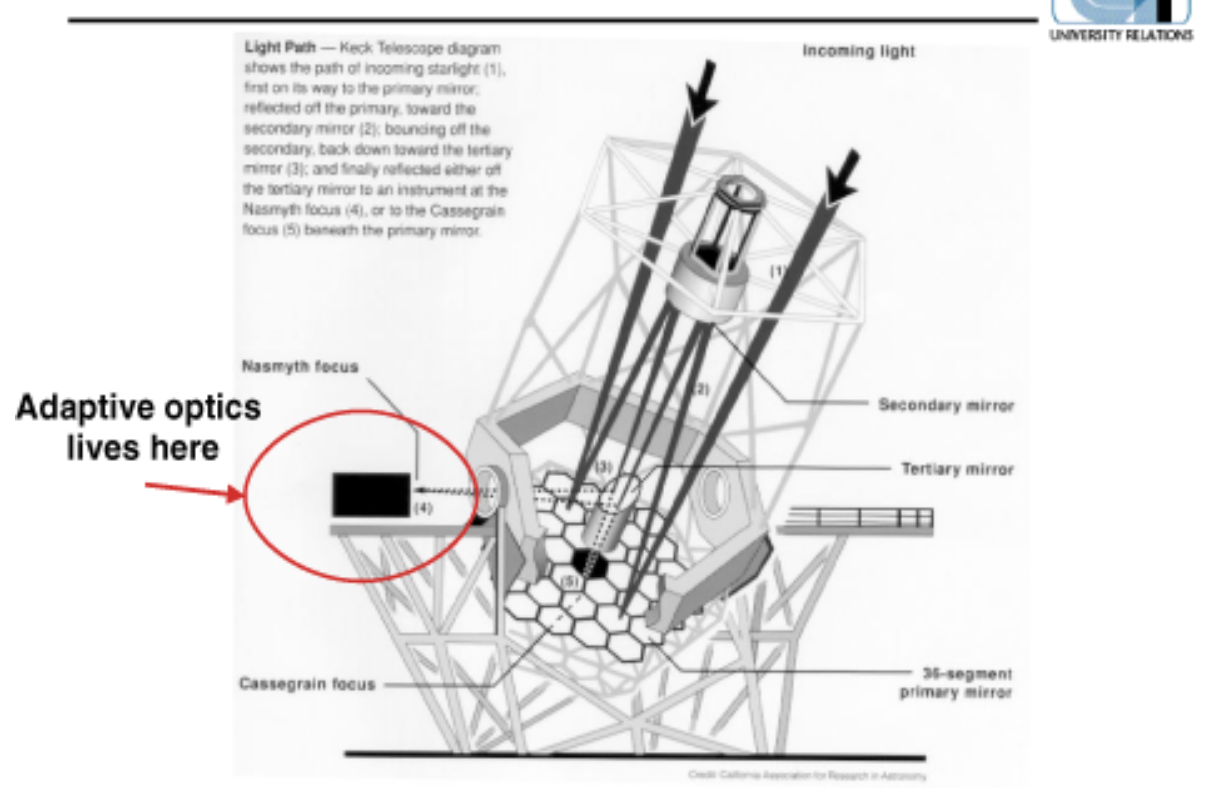

\section{Keck primary mirror before final ring} of segments was installed

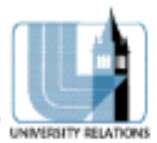

Nasmyth platform

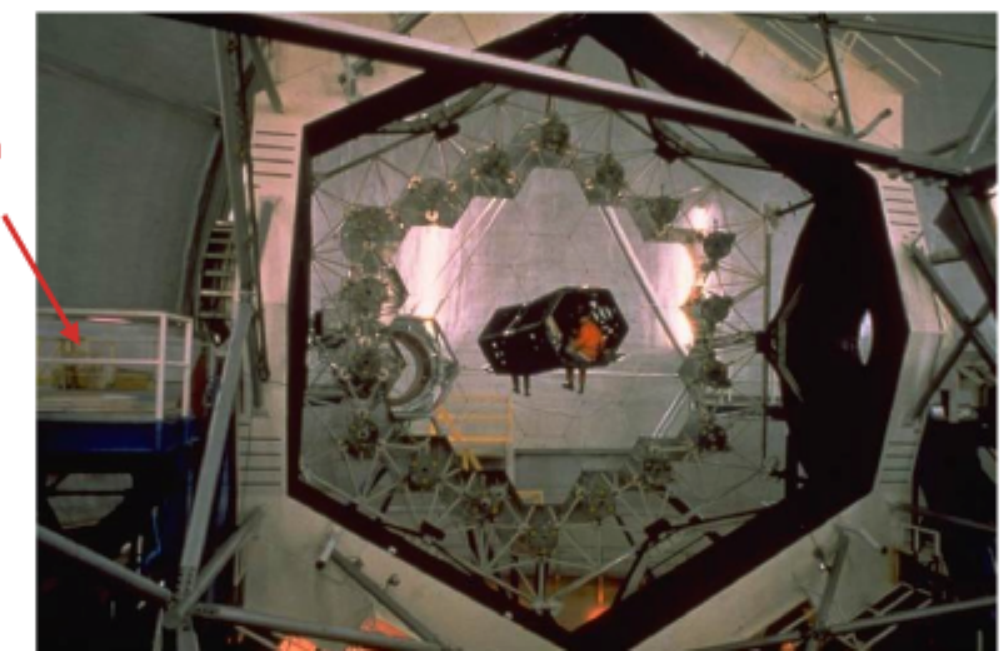




$$
\text { A S T R O N O M I C A L I MA G I N G }
$$

\section{Nasmyth platform enclosure : a meat locker!}

- Temperature-controlled via glycol cooling (some say it's a morgue)

- Rule in the dome: anything emitting more than $100 \mathrm{~W}$ has to be glycol-cooled

- Reason: "dome seeing" has been responsible for much of the poor image quality in past

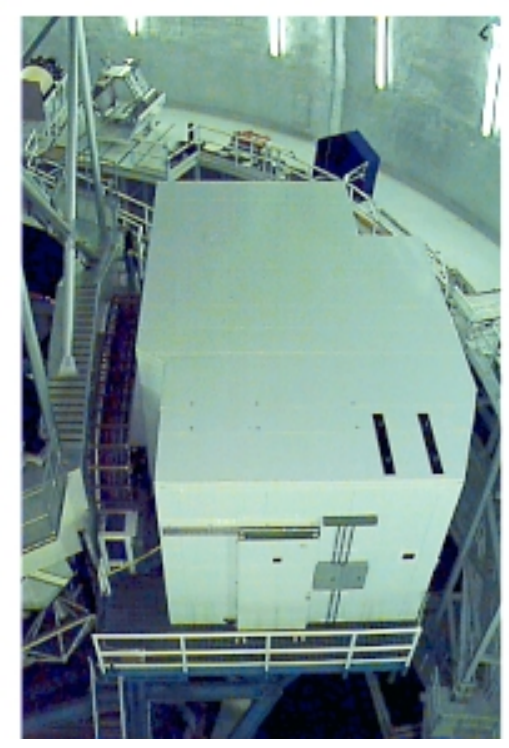

\section{Path of light to science camera}

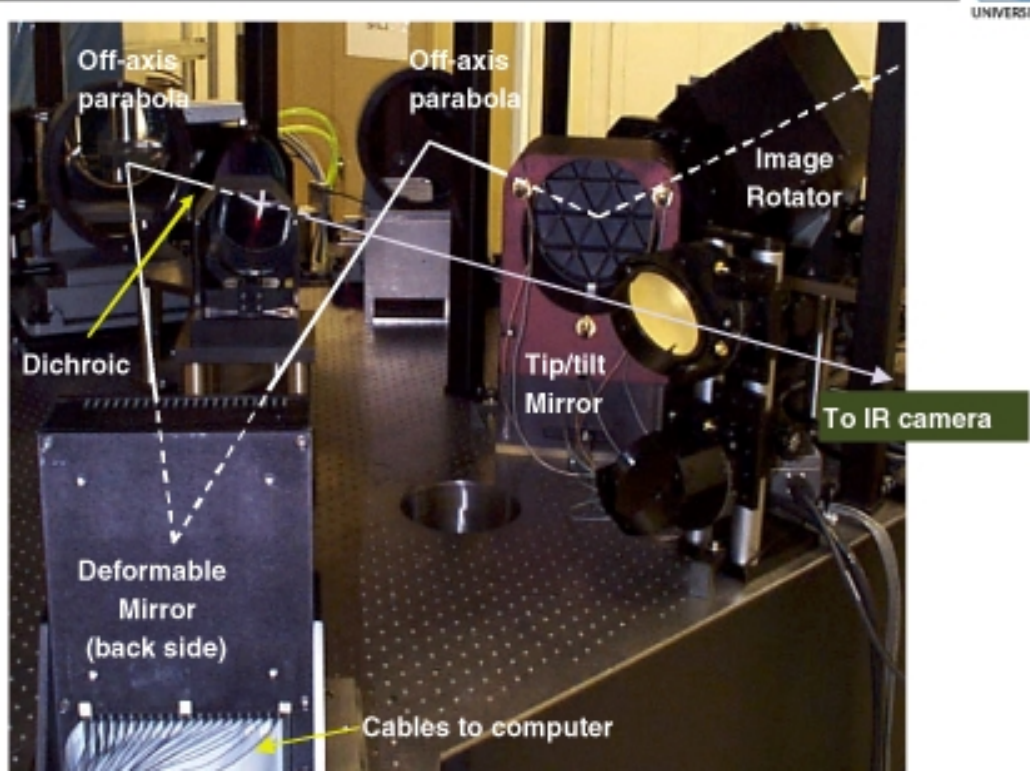


A S T R O N O M I C A L I M A G I N G

"First Light" science results

- Performance of AO system on bright stars

- Detail on the surface of Saturn's moon Titan

- Spectacular images of storms on Neptune

- Motion of stars within $\mathbf{0 . 3}$ parsecs of the black hole at the center of our Galaxy

\section{System performance on bright stars is spectacular!}

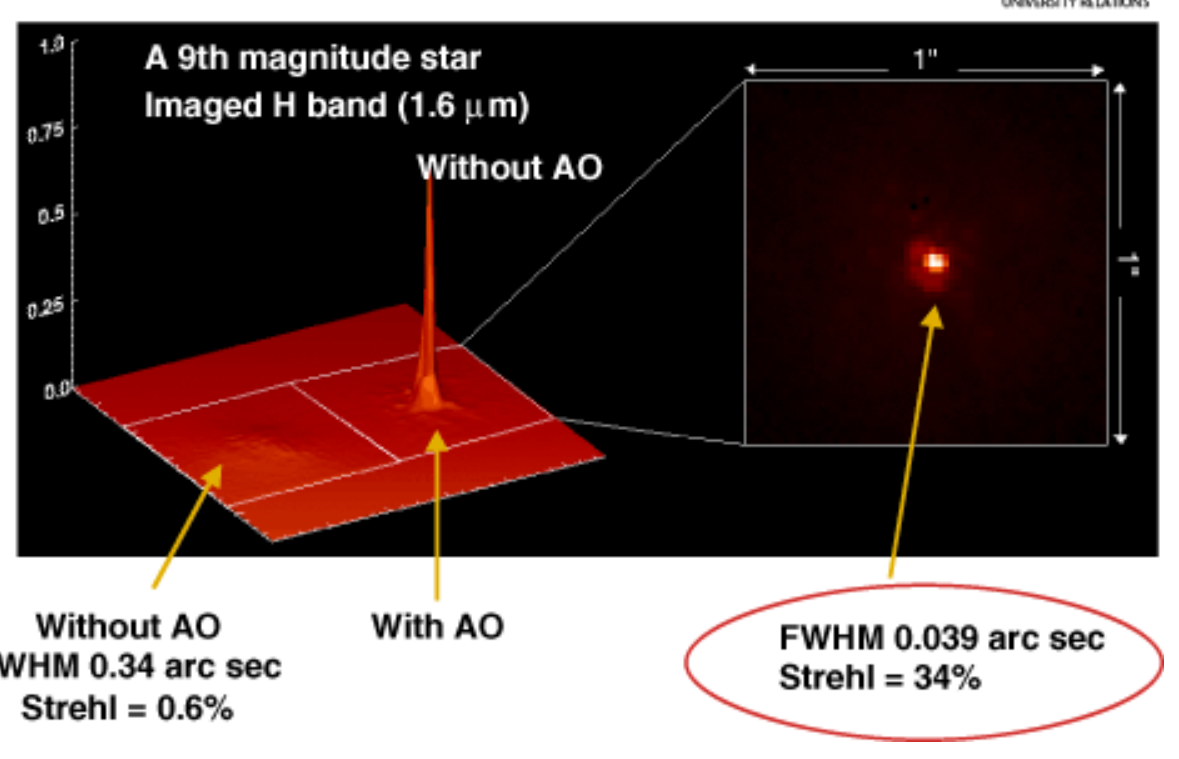




$$
\text { A S T R O N O M I C A L I MA G I N G }
$$

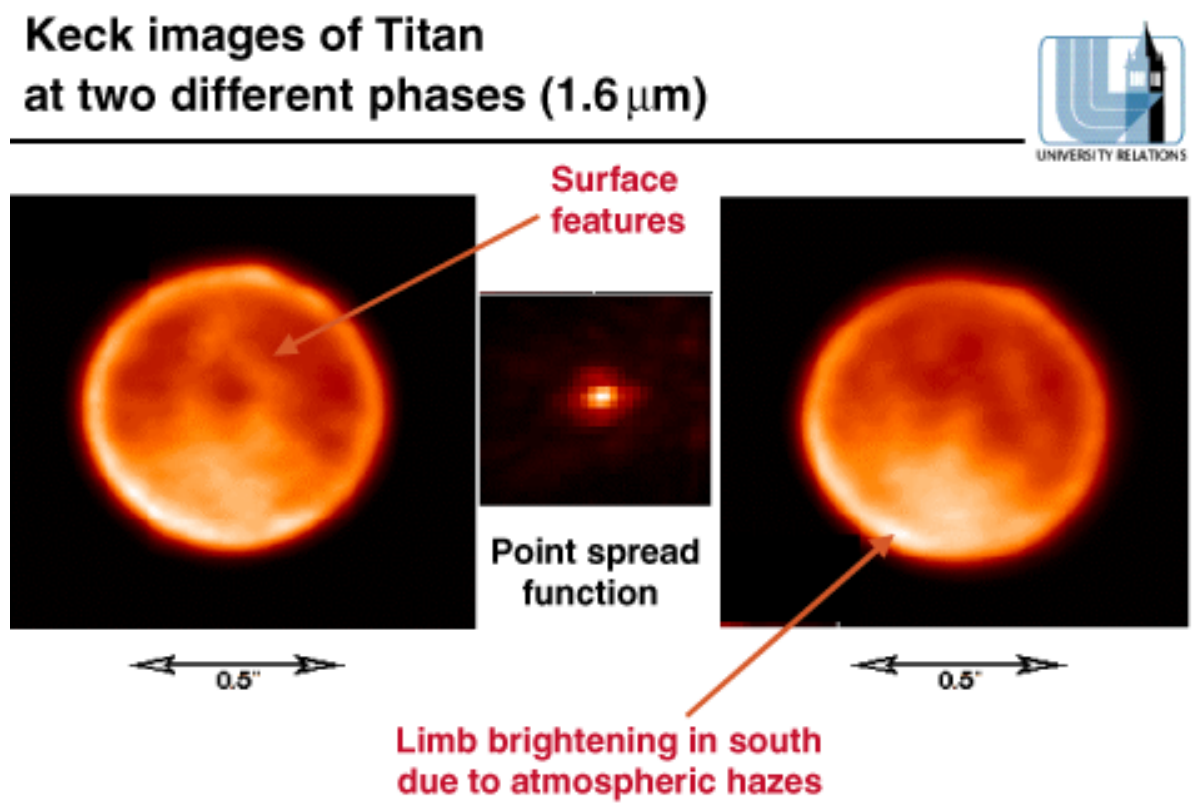

S. Gibbard, B. Macintosh, C. Max, I. de Pater (UCB)

\section{Neptune at 1.6 microns}

Without adaptive optics

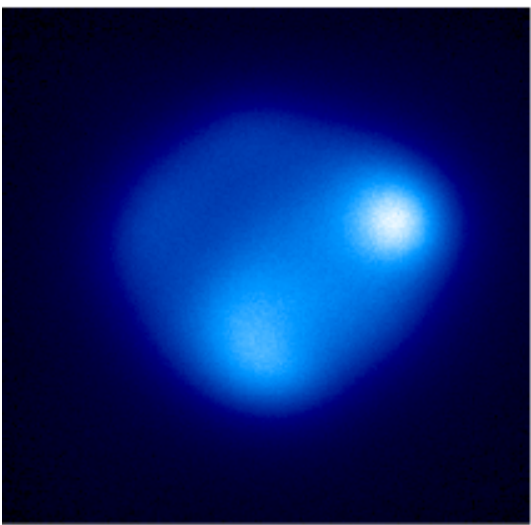

S. Gibbard, B. Macintosh, C. Max, I. de Pater (UCB)
With adaptive optics

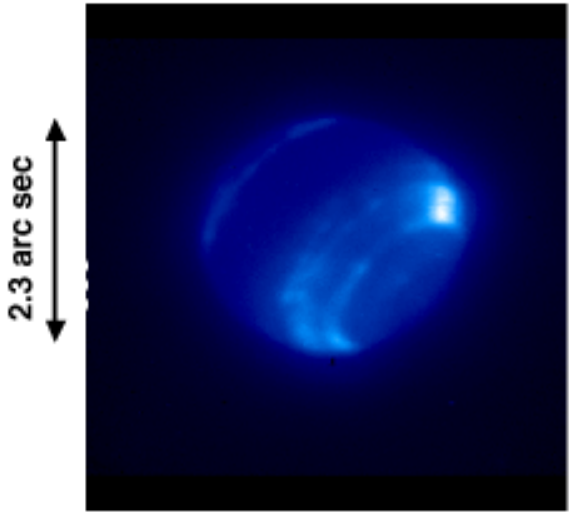


A S T R O N O M I C A L I M A G I N G

\section{Details of Neptune's bright storm at a scale of $400-500 \mathrm{~km}$}
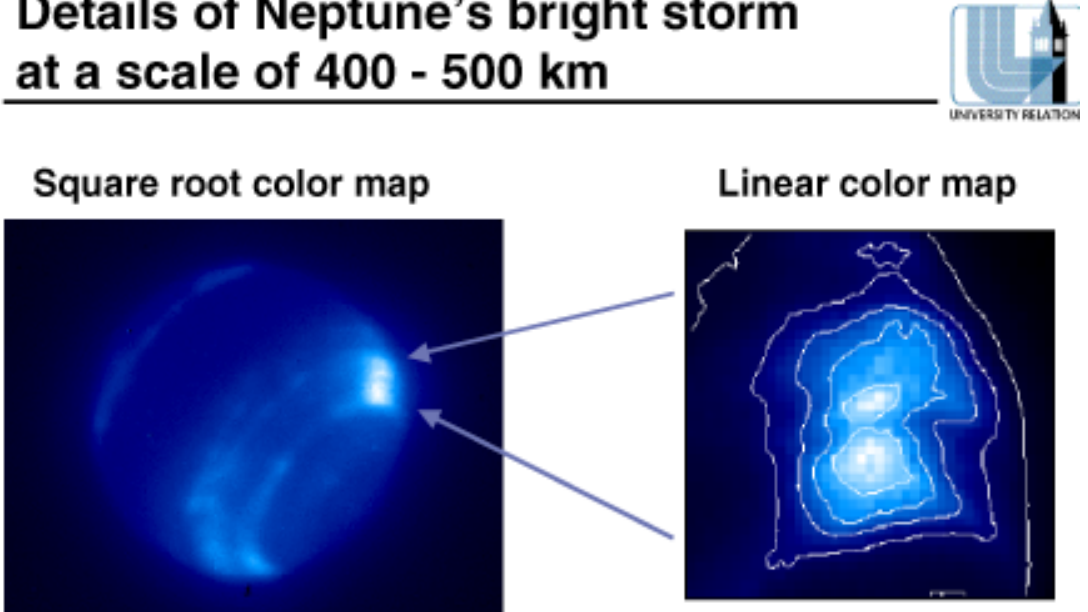

Each pixel is 0.017 arc sec $\Delta x=\mathbf{3 7 5} \mathbf{~ k m}$ at Neptune

\section{Hubble Space Telescope image of Neptune}

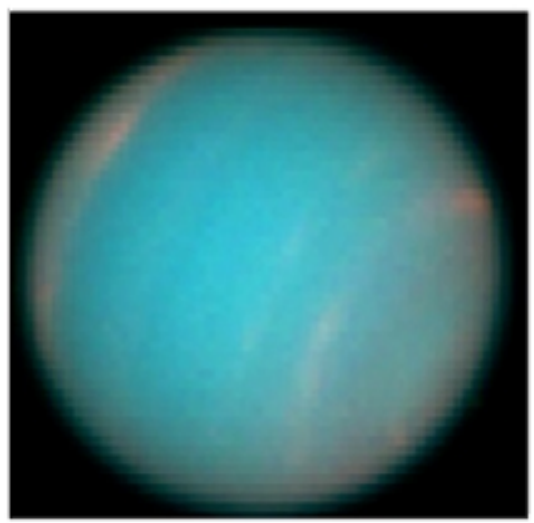




$$
\text { A S T R O N O M I C A L I M A G I N G }
$$
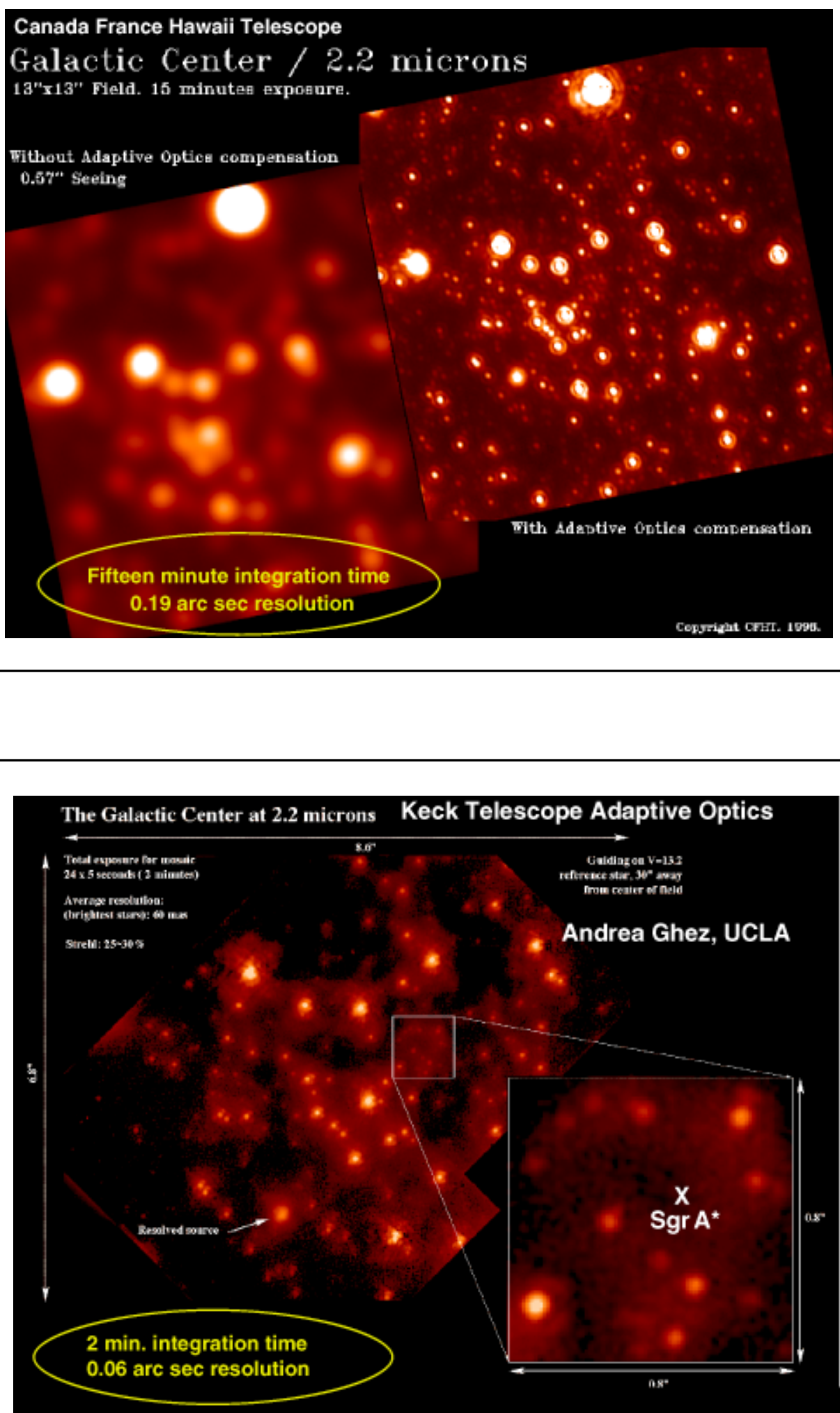


\section{Summary}

- Keck AO system is the most ambitious of the new generation of large telescopes ( $8-10 \mathrm{~m}$ diameter mirrors)

- Keck AO performance is truly outstanding:

- Without AO: $\quad$ FWHM $\sim 0.6$ arc sec

- With AO: $\quad$ FWHM $\sim 0.04$ arc sec (!)

- Better than Hubble Space Telescope

- Spatial resolution $4 \mathrm{X}$ better than HST in infra-red

- $10 \mathrm{~m}$ telescope diameter $\Rightarrow$ can detect fainter sources

- Spectroscopy will be spectacular

This really is the dawn of a new age for astronomy!

\section{Coda: Overview of new NSF Center for Adaptive Optics}

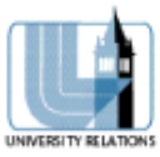

- NSF Science and Technology Centers:

- Multidisciplinary, education \& outreach, industrial partners

- Competition last year: several hundred applied, 5 selected

- Center for Adaptive Optics:

- Lead by UC Santa Cruz - home of UC Observatory and Lick

- Jerry Nelson PI (inventor of Keck Telescope)

- Very strong participation by LLNL

- Many other institutions are partners

\section{Adaptive optics for astronomy and vision science}




\section{Adaptive Optics Provides the Highest Resolution Images of the Living Retina}

$>3 x$ increase in transverse resolution
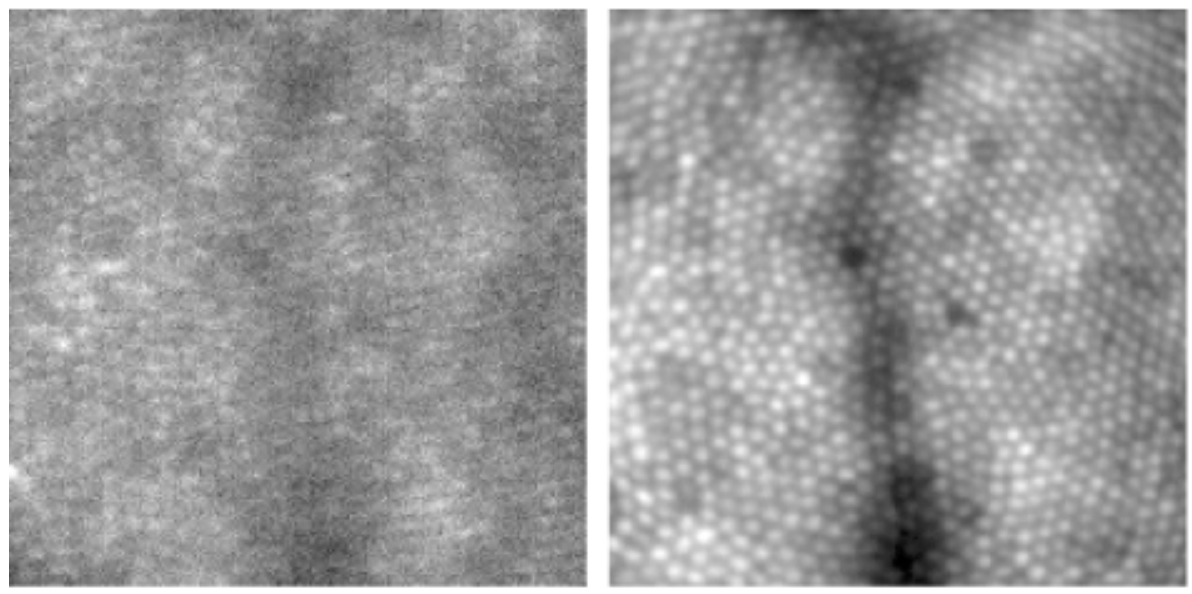

Without AO

With AO: resolve individual cones

\section{Human Color Vision: huge variability from one individual to the next!}
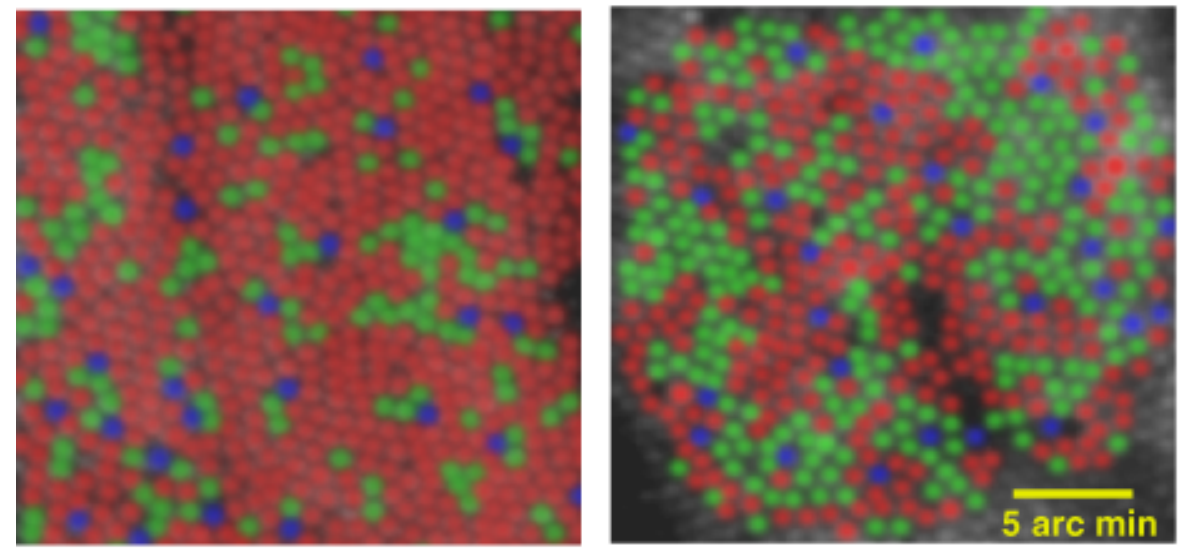

First images of the human trichromatic cone mosaic, Roorda and Williams, Nature, 1999. Note how different the two cone patterns are! 


\title{
Searches for Low Mass Extra-solar Planets
}

\author{
Jian Ge E Dave Erskine, LLNL
}

One of the most exciting fields in astronomy is the search for extra-solar planets by measuring the extremely small Doppler velocity shifts on starlight caused by planets. However, two main factors are preventing current planet searches from detecting low mass extra-solar planets such as Saturn-like and Earth-like planets. One factor is related to the unstable and irregular point spread functions with the current seeing-limited echelle spectrographs. The other is related to the intrinsic turbulence and oscillations in the star's photosphere. These two factors limit the radial velocity resolution beyond $\sim 3 \mathrm{~m} / \mathrm{s}$ (Marcy \& Butler 1998).

The novel spectrally dispersive interferometer, or fringing spectrometer (Erskine \& Ge 1998; 1999), developed by us at LLNL, provides one solution to the current problems. The simple and stable instrument responses enables significant reduction of radial velocity error to less than $\sim 1 \mathrm{~m} / \mathrm{s}$. The laboratory-based testing with this instrument has demonstrated a $0.76 \mathrm{~m} / \mathrm{s}$ resolution capability, more than three times better than the current stateof-the-art. The instrument is being coupled to the Leuschner 30inch telescope for planet searches- similar velocity resolution for starlight is expected soon. Once candidate stars showing a few $\mathrm{m} / \mathrm{s}$ velocity variation are identified with this instrument, further observations with another LLNL instrument, which is a novel adaptive optics very high resolution spectrograph, will follow up to monitor their stellar photosphere activity. This will help the detection of low mass extra-solar planets. The combination of the dispersive interferometer and the adaptive optics very high resolution spectrograph will be the most promising way for low mass extra-solar planet searches in the next decade. 
Presentation

at 99 CASIS

Workshop at

LLNL

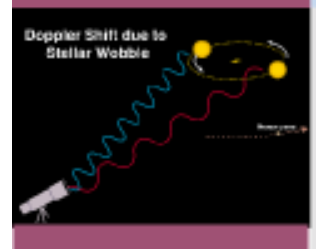

UCRL IL10168

\title{
Searches for Low Mass Extra-solar Planets
}

\author{
Nov. 12, 1999
}

\section{Jian Ge \& Dave Erskine}

Lawrence Livermore National Laboratory

Collaborators:

Gibor Basri(UCB), Bruce Macintosh (IGPP), MikeRushford (LLNL), Mike Lloyd-Hart, Roger Angel (UA)
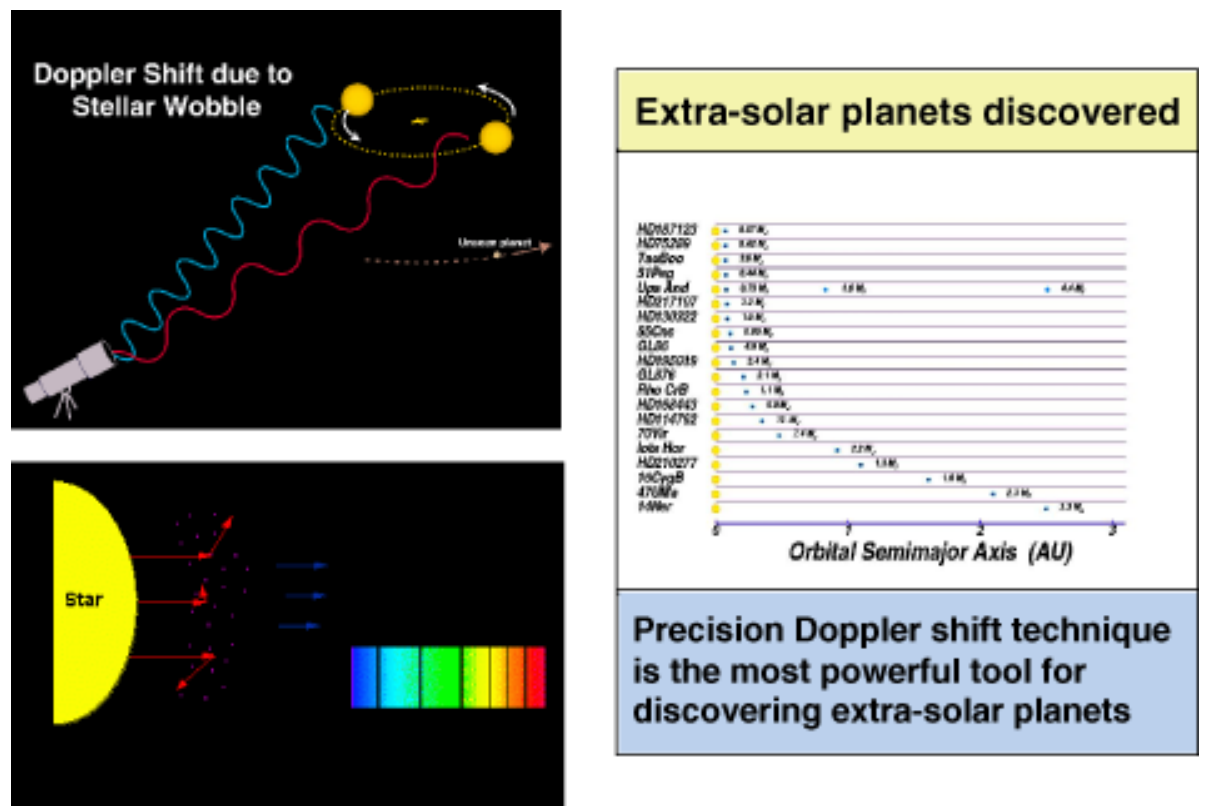


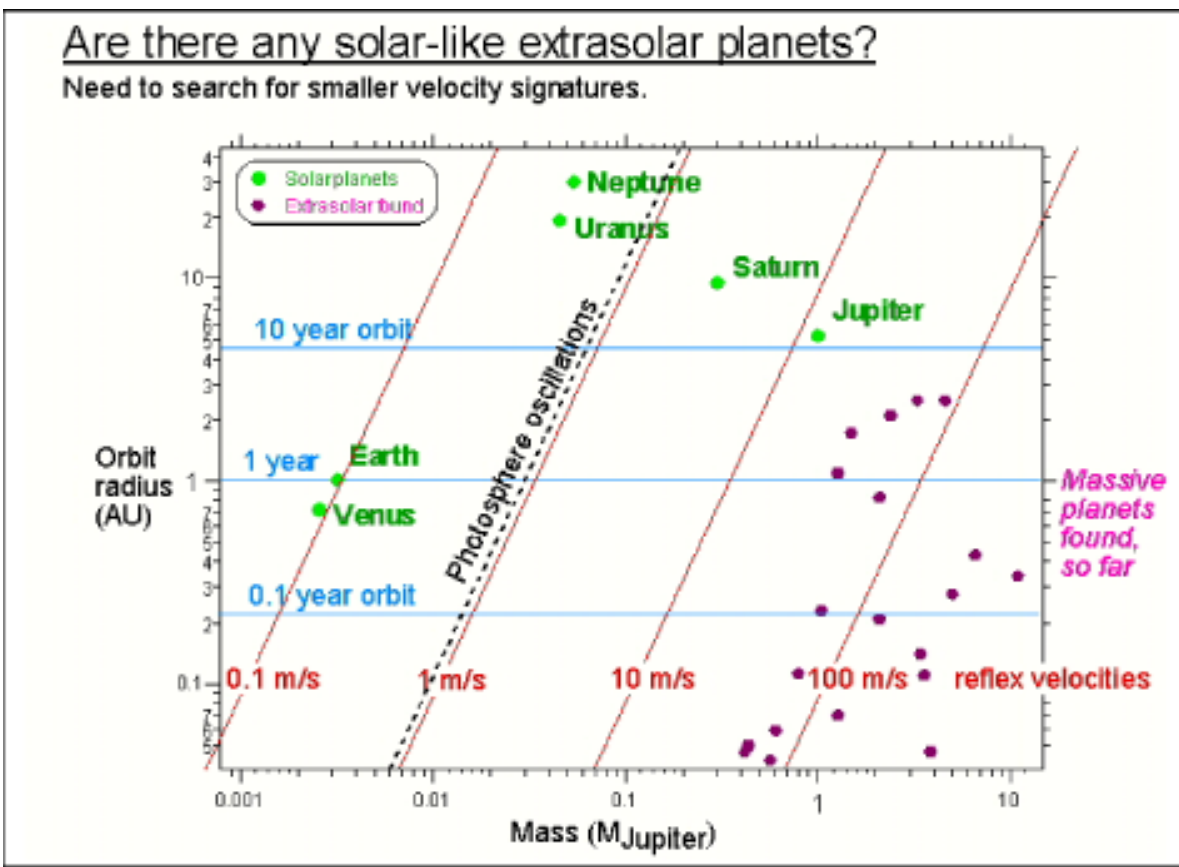

Doppler velocity precision $<3 \mathrm{~m} / \mathrm{s}$ in order to detect solar-like extra-solar planets

\section{Current State-of-the-art Doppler Radial Velocity Precision}

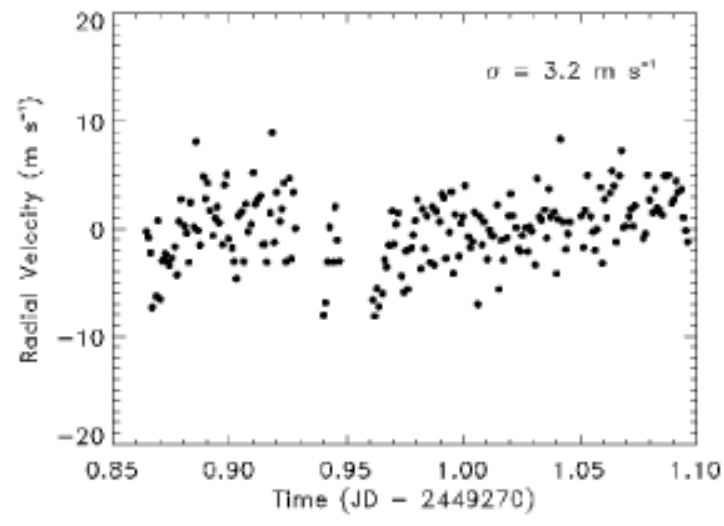

Current radial velocity precision barely allows detection of Jupiter-like planets, not Saturn-like \& Earth-like planets 
$\begin{array}{lllllllllllllllllll}A & S & T & R & O & N & O & M & \text { I } & C & A & L & \text { I } & M & \text { A } & G & \text { I } & N & G\end{array}$

The simplest instrument response of an interferometer offers more precise measurements of slight Doppler shifts
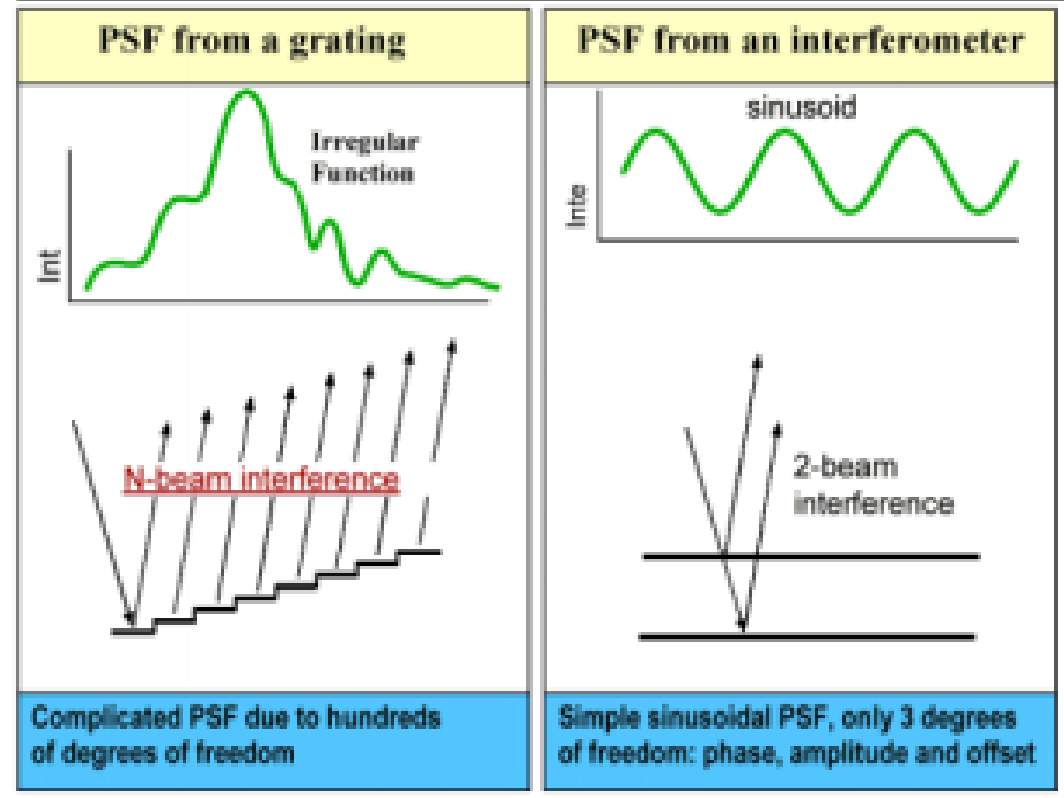

Dispersive Interferometer (or fringing spectrometer) combine best features of interferometer and grating spectrometer

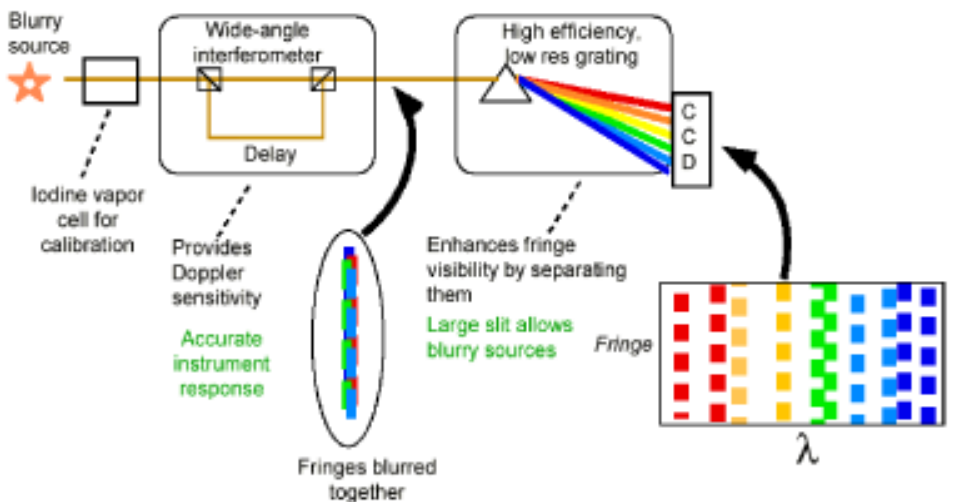

- The interferometer produces pure and simple sinusoidal fringes (PSFs)

- The spectrometer disperses white fringes into different color channels to increase fringe visibility for precision phase measurements 


\section{Prototype dispersive interferometer for initial lab testing}

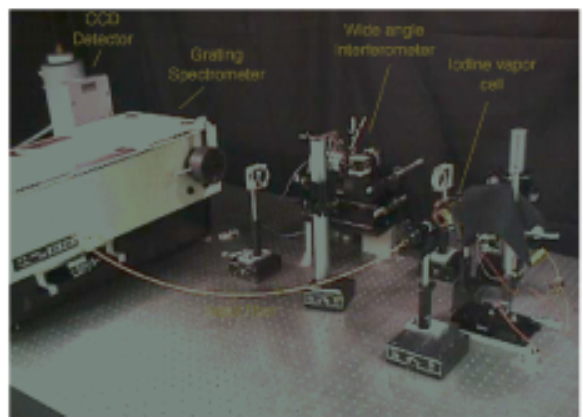

Solar fringing spectrum obtained by the prototype dispersive interferometer

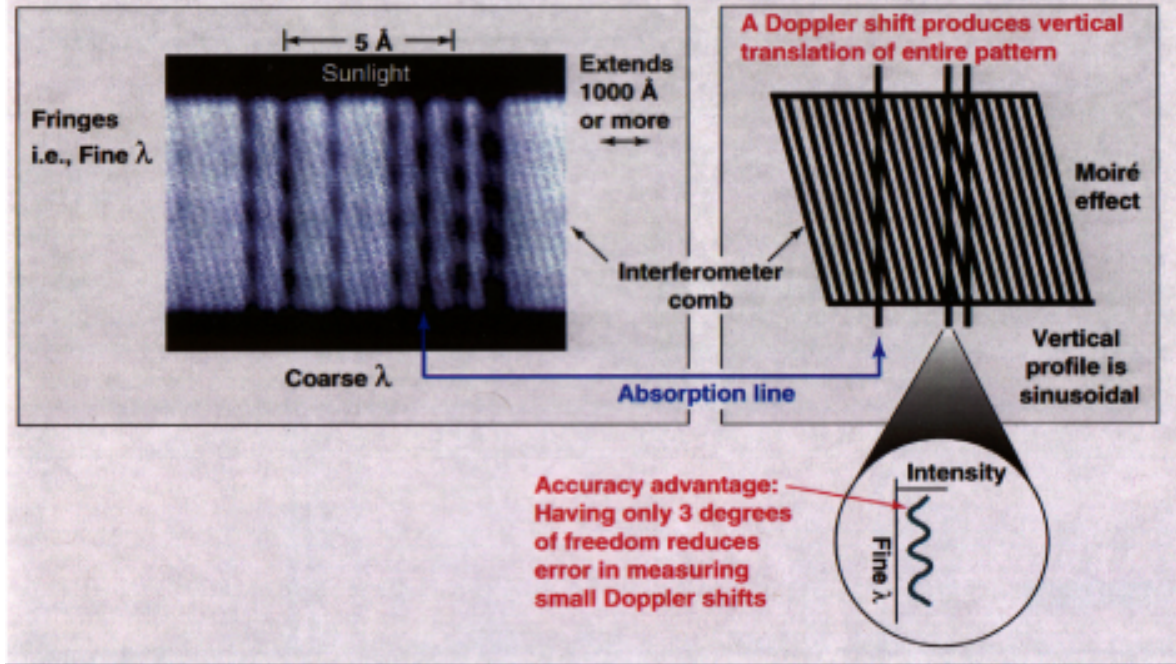

Solar absorption line Moiré fringes are used to measure tiny phase shifts caused by radial velocity variations 


\section{Observations of the Earth's Diurnal Motion and Solar 5 minutes'} Oscillation with the Prototype

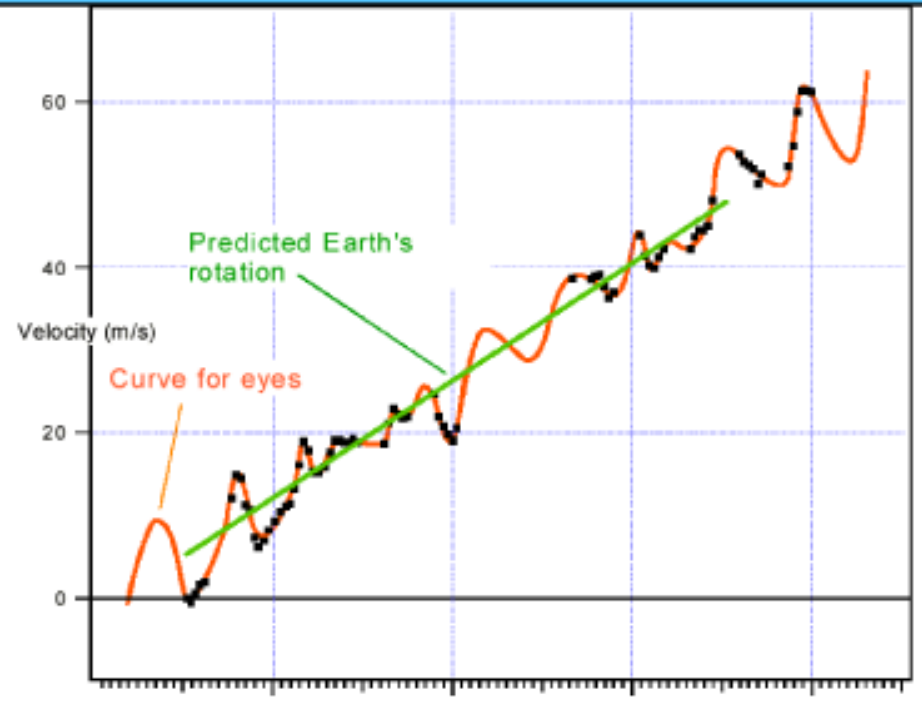

11:50 AM

12-00 PM

12:10 PM

12:20 PM

We needs further observations to confirm the solar oscillation detection due to the unstable heliostat setup during the measurements

\section{Null tests: Prototype's radial velocity precision is better than current state-of-the-art}

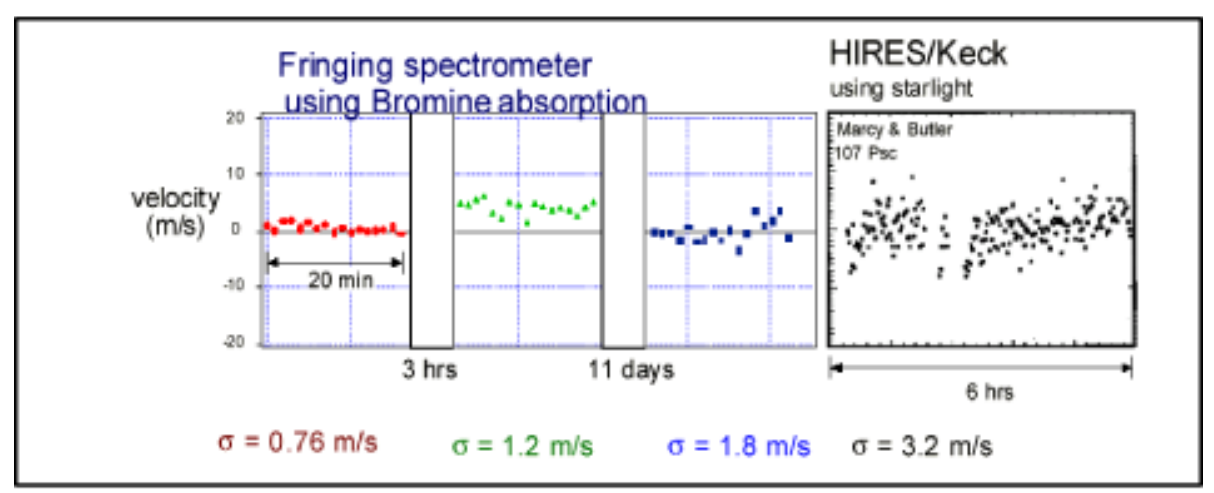

- Short term radial velocity precision already $3 x$ better

- Additional controls on interferometer cavity etc. will reduce systematic errors for better long term precision 
A S T R O N O M I C A L I M A G I N G

\section{A new fiber-fed dispersive interferometer is ready for starlight testing}
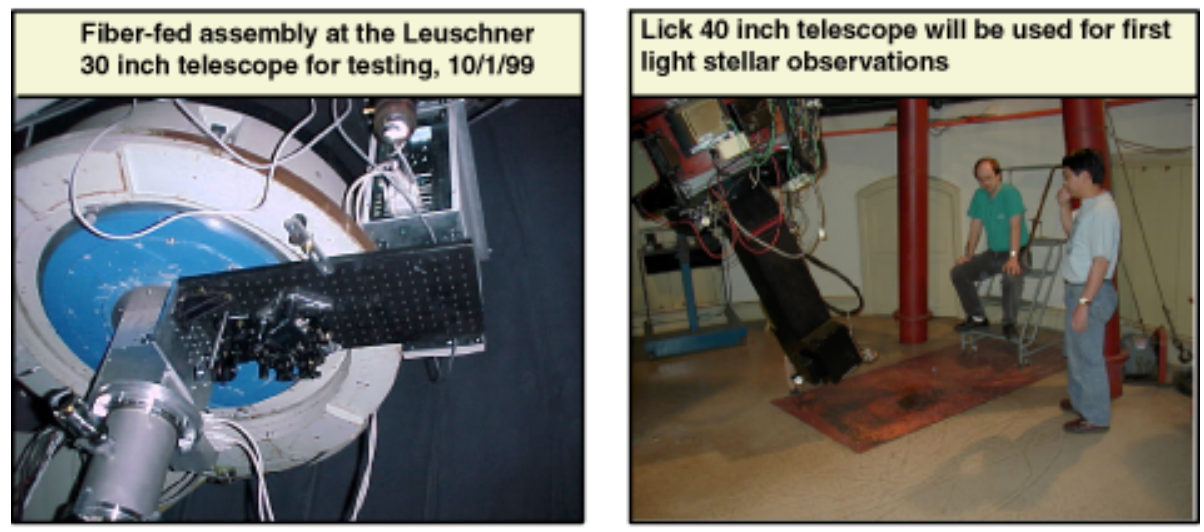

A survey for low mass extra-solar planets will start at the Lick 40 inch in December 1999

Stellar activity-related radial velocity variations limit Doppler precision for low mass planet searches
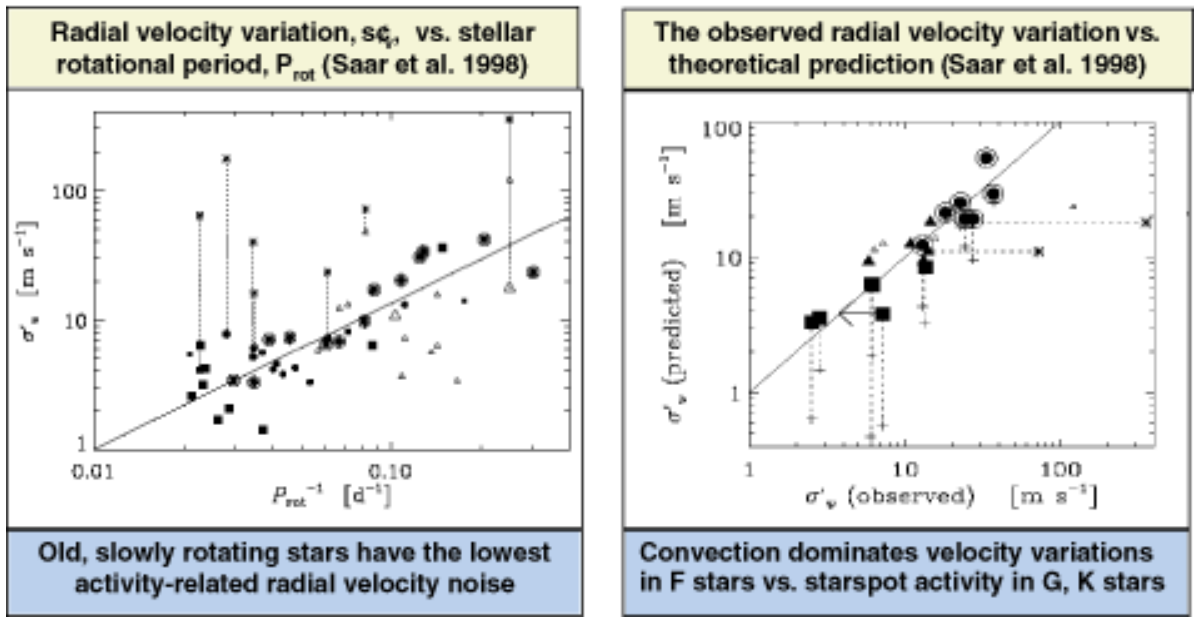
A S T T R O N O

Study of stellar absorption line profile is the key to understand different contribution to stellar radial velocity variation
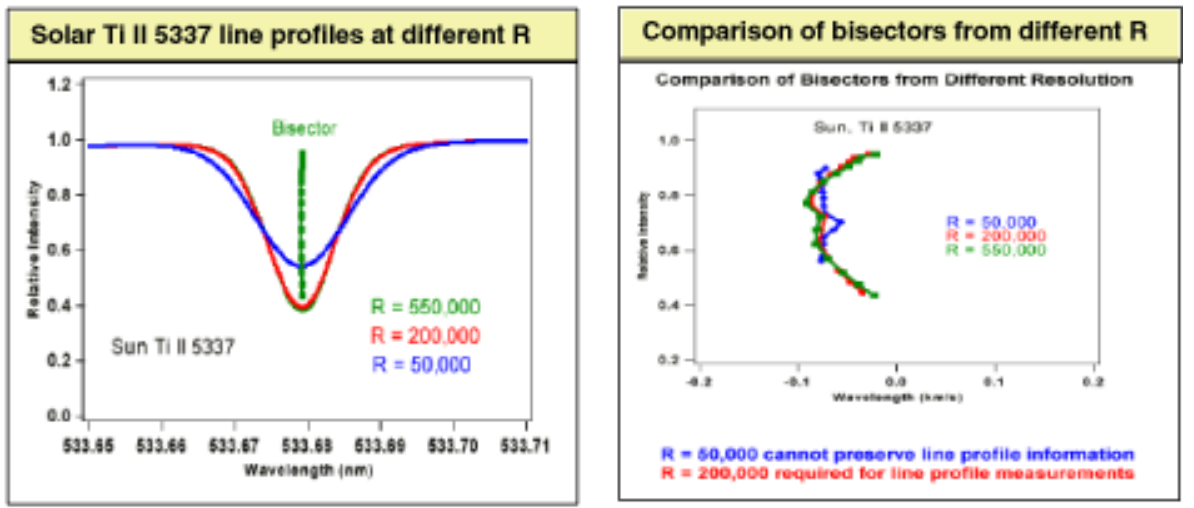

- At R 200,000, line asymmetries can be measured and used to disentangle stellar intrinsic velocity perturbations from planet induced radial velocity changes

- Doppler precision of - $1 \mathrm{~m} / \mathrm{s}$ achievable with the correction for stellar activity

Setup of a New AO Optical Cross-dispersed Echelle Spectrograph at Steward Observatory, Nov. 1998, R = 200,000, 0.35-1.0 $\mu \mathrm{m}$ (Ge et al.)

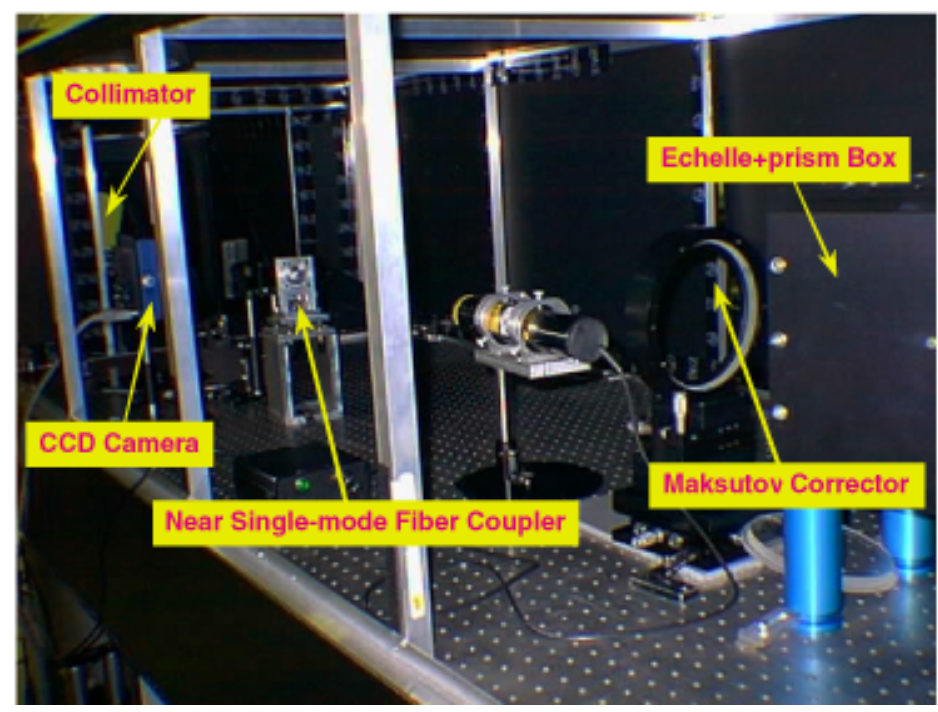


Solar Spectrum Obtained with the AO Spectrograph and 2Kx4K CCD Camera at Steward Obs., May 99 (Ge et al.1999)
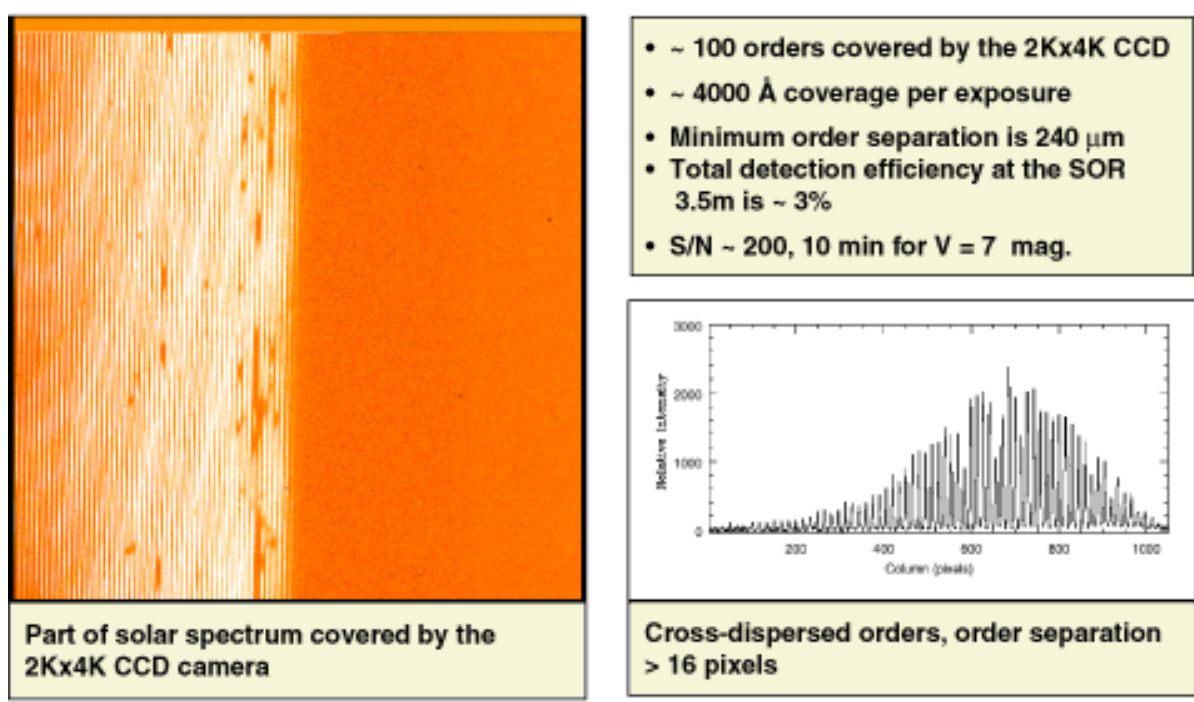

- $\mathrm{S} / \mathrm{N} \sim 200,10 \mathrm{~min}$ for $\mathrm{V}=7 \mathrm{mag}$.

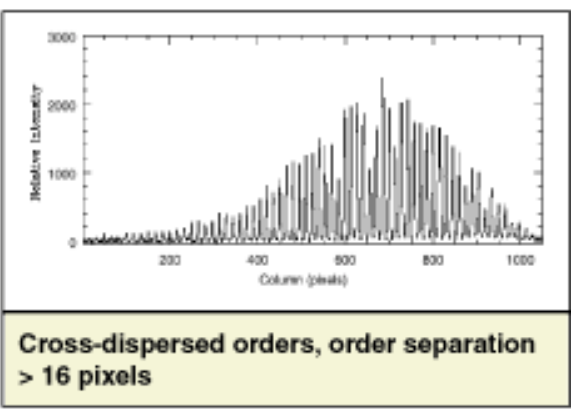

The spectrograph will be commissioned at the SOR $3.5 \mathrm{~m}$ telescope in Dec. 99

A compact IR immersion echelle spectrograph with adaptive optics (LISPEC) is being developed at LLNL to achieve $R=100,000-300,000$ in 1.2-5.5 $\mu \mathrm{m}$ (FY99-FY02)
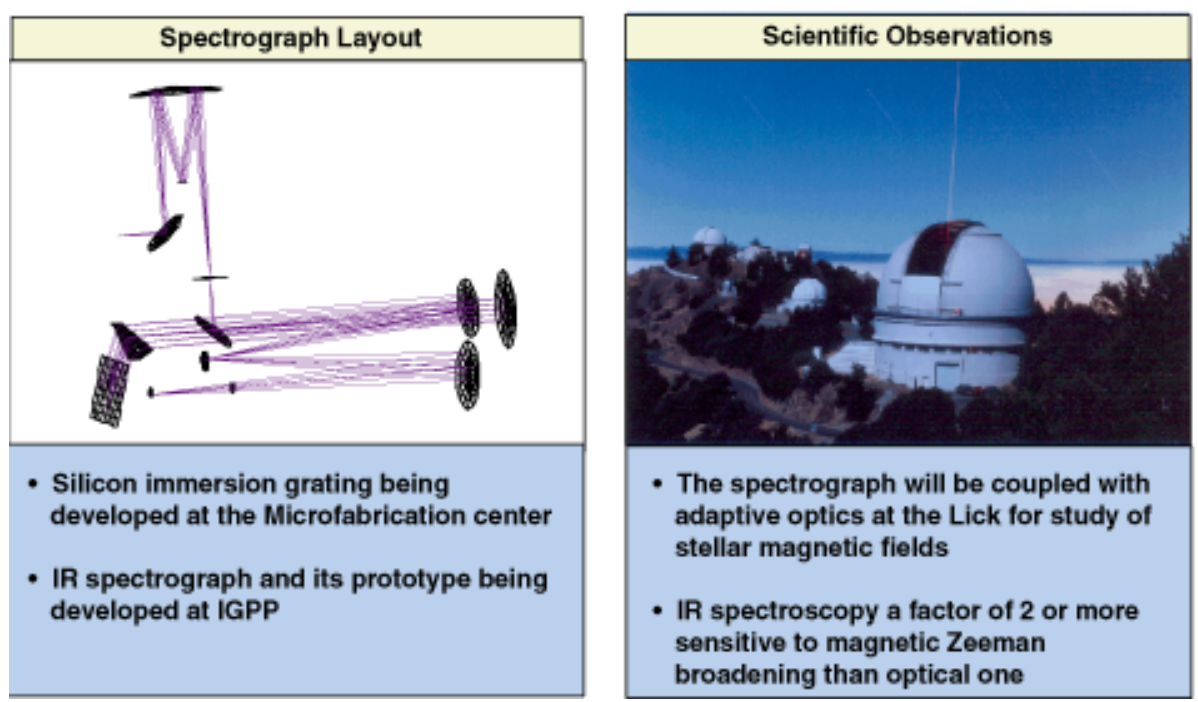


\title{
Demonstration of a Diffractive Telescope
}

\author{
Sham Dixit \\ Co-authors: \\ Mike Rushford, Ian Barton, Frank Patterson, Leslie Summers, John Prior, \\ Holly Cagle, Jerry Britten, Katy Lu, Mike Perry and Rod Hyde
}

We present results of the imaging and color correction properties of a telescope that uses diffractive fresnel lenses. The theory behind a color correction of a diffractive telescope will be discussed. This will be followed by a description of the fabrication of the Fresnel lenses and the fielding of the telescope. Images of the planets in the solar system taken using the telescope will be presented.

\section{Demonstration of a diffractive telescope}

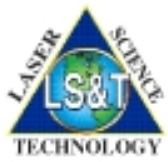

Sham Dixit

Mike Rushford, Ian Barton, Frank Patterson, Leslie Summers, John Prior Holly Cagle, Jerry Britten, Katy Lu, Mike Perry and Rod Hyde

Presentation to:

CASIS Workshop

November 11, 1999 


\section{Eyeglass is a new type of space telescope}

Contains two cooperating spacecraft

- Separated by a few kilometers

- Tied together via micro-gee propulsion

Magnifying Glass: Thin diffractive lens

- Provides the large aperture

- Lightweight and easy-to-deploy

- With very loose surface tolerances

Eyepiece: Compact, meter-class, space telescope

- Moves along focal surface, collecting light

- This is a conventional, low-risk, vehicle

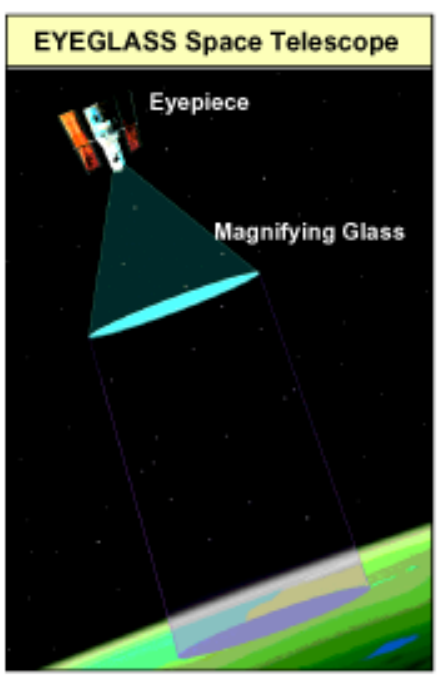

\section{Why Do Things This Way?}

- Thin lenses have much looser tolerances than mirrors

- Surface ripples on a mirror are optically doubled

- Ripples on a thin lens are optically cancelled

- This effect is best for weak, low F\#, lenses

- Surface tolerances are $\sim 1 \mathrm{~cm}$, not $\sim 500$ angstroms

- An F/100 lens is 160,000 times better than a mirror

- We must use diffractive lenses, not refractive ones

- A conventional lens is too thick and heavy

- A diffractive lens is very much thinner and can be made lightweight and foldable
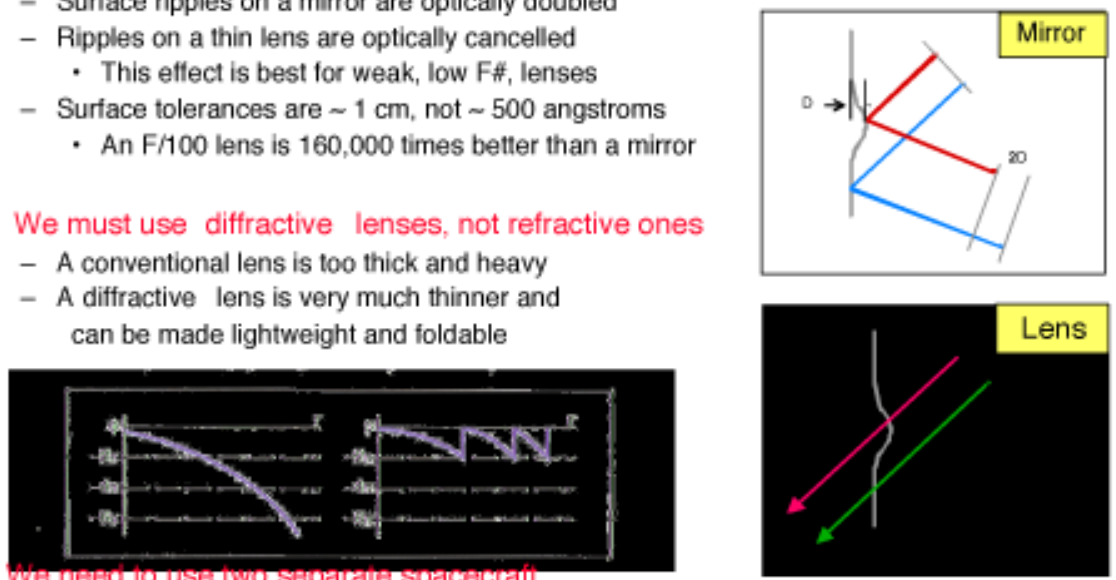

- Focal length is kilometers

- Product of large aperture (for resolution) and high F/\# (for tolerance gains)

- These lengths are practical in space, by splitting the telescope into two parts 
A S T R O N O M I C A L I M A G I N G

Color correction in a diffractive telescope

Fresnel primary

Collection

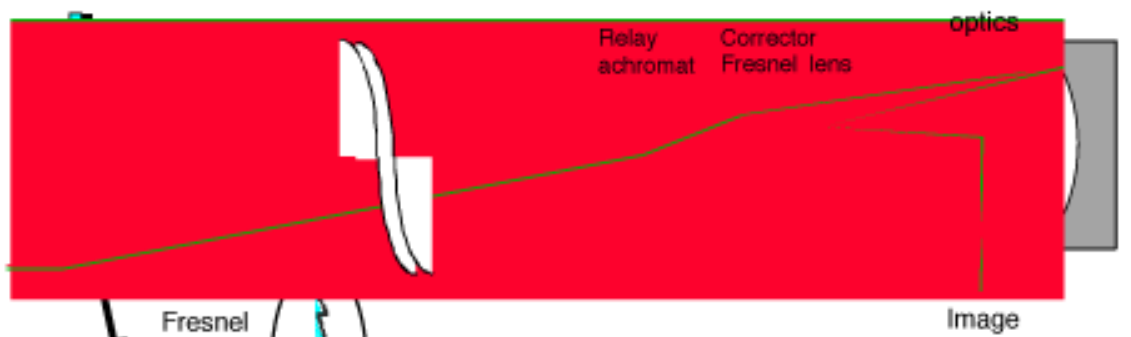

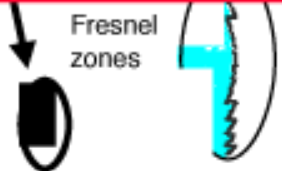

Features:

Large aperture $(25-50 \mathrm{~m})$

Long $\mathrm{f} *$

Fabricated on a thin membrane
Relay achromat images the primary onto the corrector Fresnel lens

Corrector Fresnel lens compensates the chromatic effects of the primary

Collection optics brings light to focus

\section{Characteristics of a diffractive Fresnel lens}

Example shown is for a $50-\mathrm{cm}$ diameter $\mathrm{f} / 100$ lens

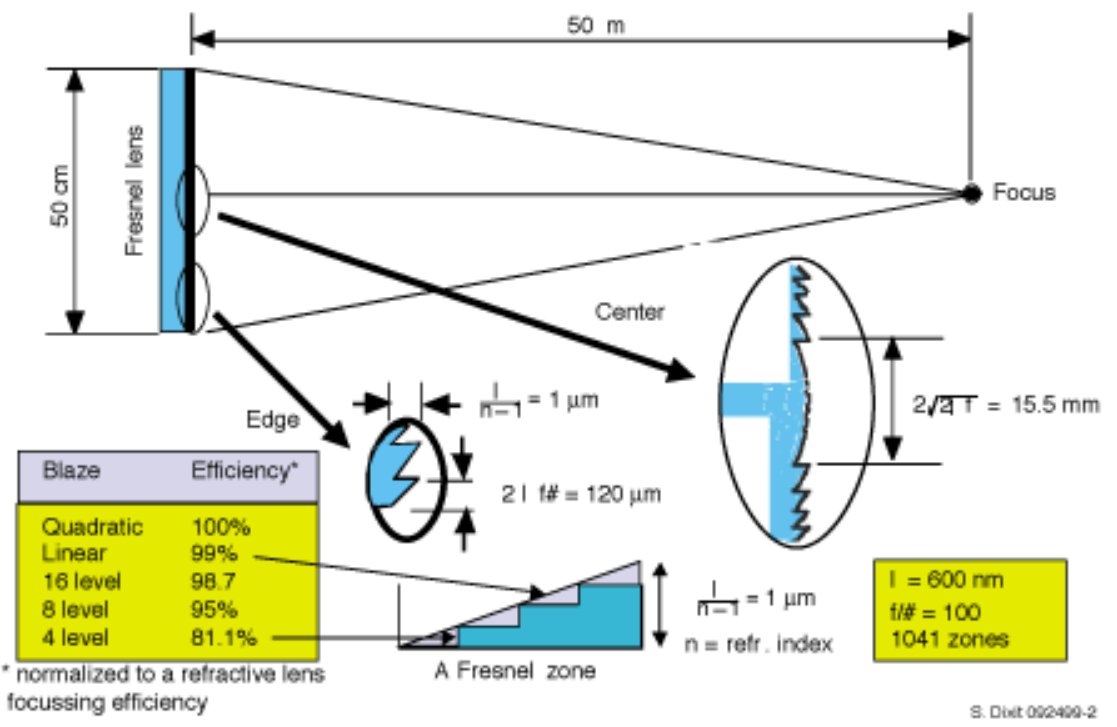


A S T R O NOM I C A L I MAG I N G

The Fresnel lens phase profile is fabricated by overlaying multiple binary mask patterns in a lithographic process

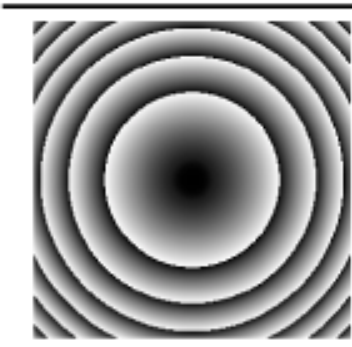

Phase profile $(\bmod 2 \pi)$

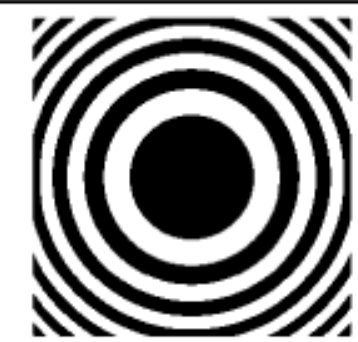

Coat with

photoresist

Substrate

phase step depth $=\mathrm{t}$

mask 1 ; etch depth $t / 2$

To achieve high

diffraction efficiency, we

need:

- high precision binary

masks ( $<1 \mu \mathrm{m}$ accuracy)

- good alignment $(<1 \mu \mathrm{m}$

precision)

- precise etch depth

control (10-20 nm)
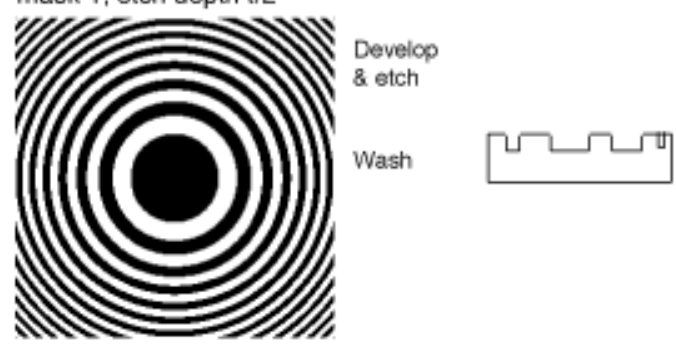

Expose with UV

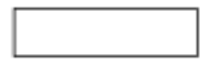

mask 2 ; etch depth $t / 4$

\section{$20 \mathrm{~cm}$ Diffractive Telescope: Hardware}
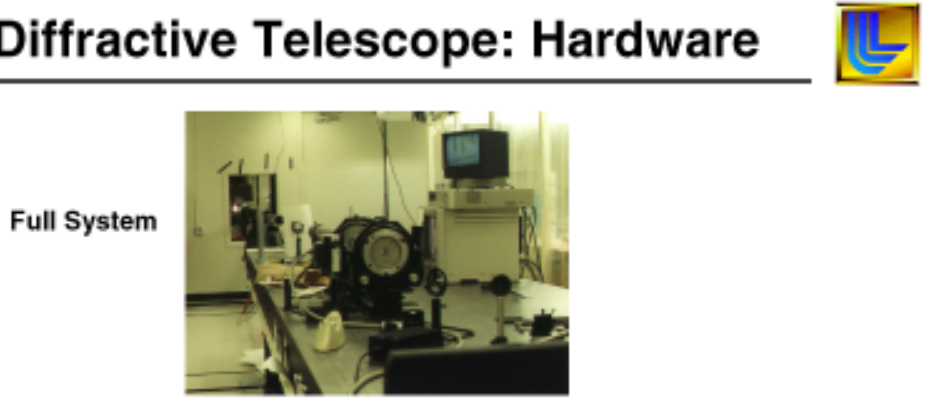

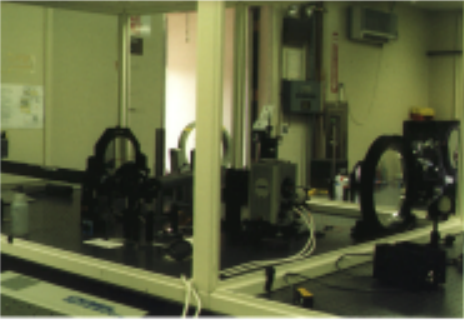

Beam Expander and Diffractive Primary

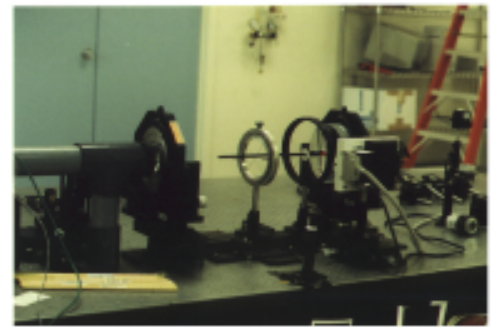

Fresnel Corrector and Schwarzschild Collector 
A S T R O N O M I C A L I M A G I N G

\section{Color Correction Has Been Demonstrated}

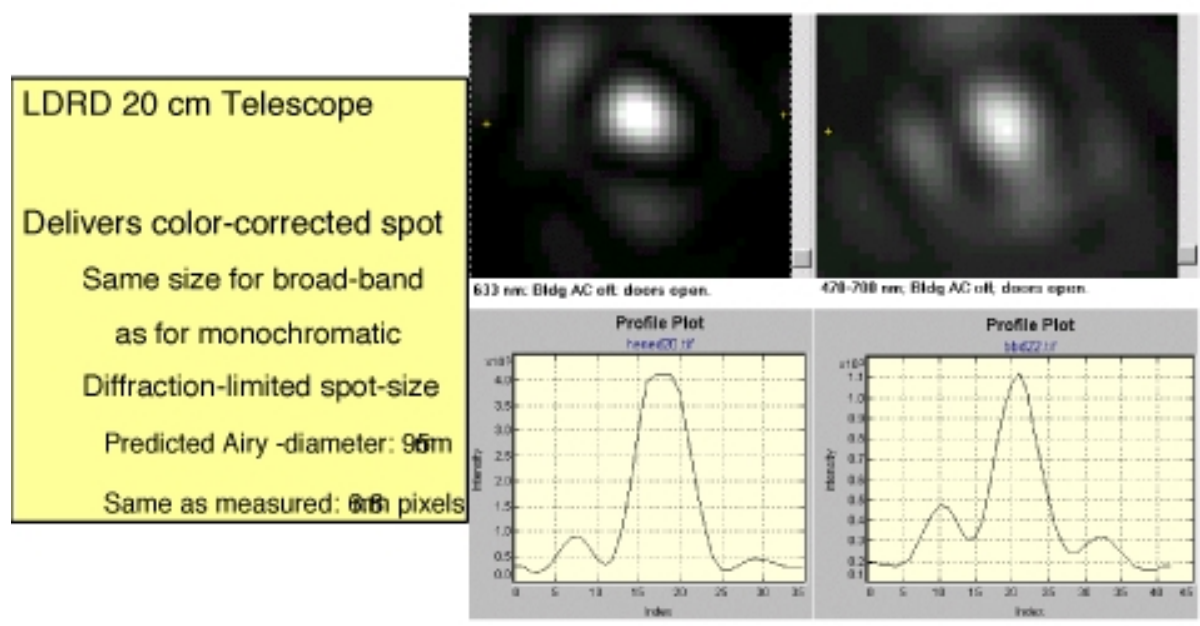

\section{Diffractive Telescope Has Taken Astronomical Images}

\section{- Despite complications}

-Telescope is immobile, with narrow FOV

-Image must be piped-in with turning mirrors

- We have obtained clear astronomical images

-White light: Moon, Jupiter, Saturn, Sun-spots

- Narrow band: Solar flares

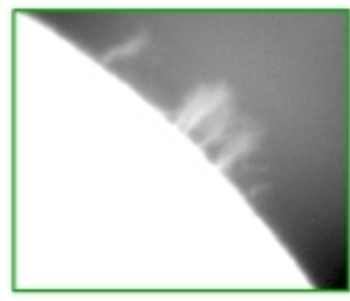

Solar Flares

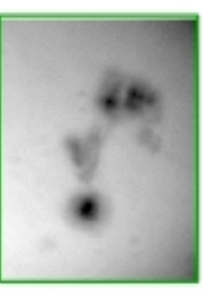

Sun-Spots

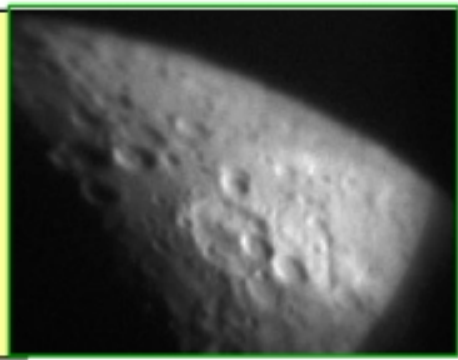

Lunar Surface

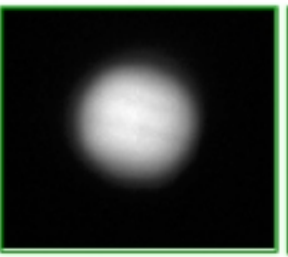

Jupiter

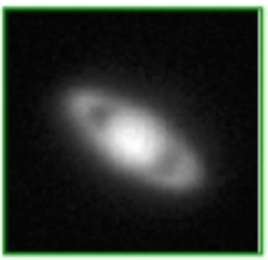

Saturn 
We have successfully fabricated the binary mask for the 50-cm Fresnel lens

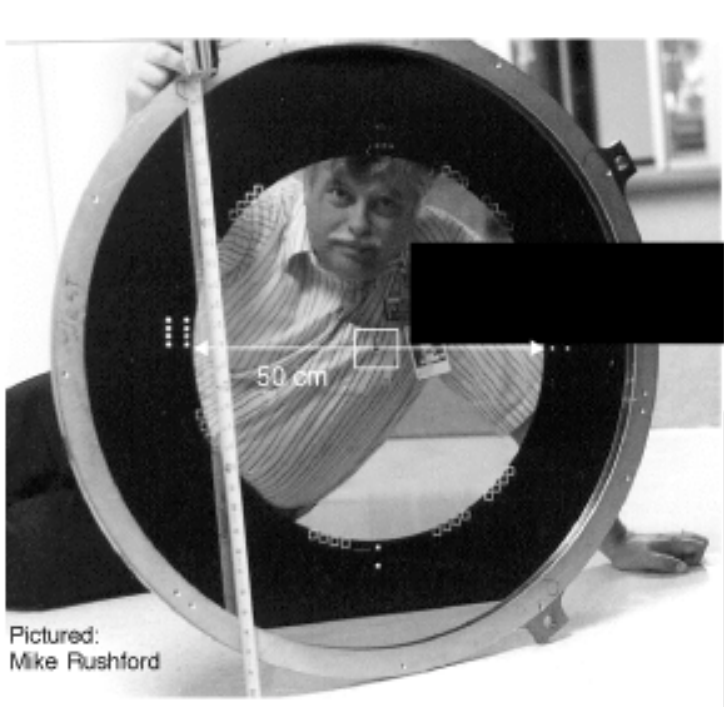

Expanded view of the center of the mask

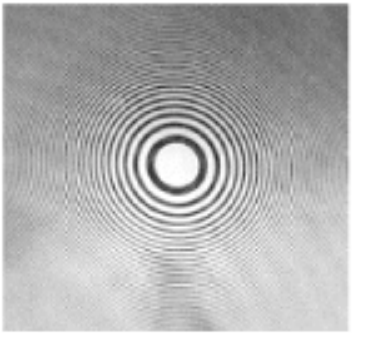

Mask parameters:

Aperture diameter $=50 \mathrm{~cm}$

Focal length $\quad=50 \mathrm{~m}$

Design wavelength $=0.6 \mu \mathrm{m}$

Central zone dia. $=11 \mathrm{~mm}$ Last zone width $\quad=60 \mu \mathrm{m}$ Number of zones $=1041$

Nova laser bay is being used to field the $50-\mathrm{cm}$ diffractive telescope

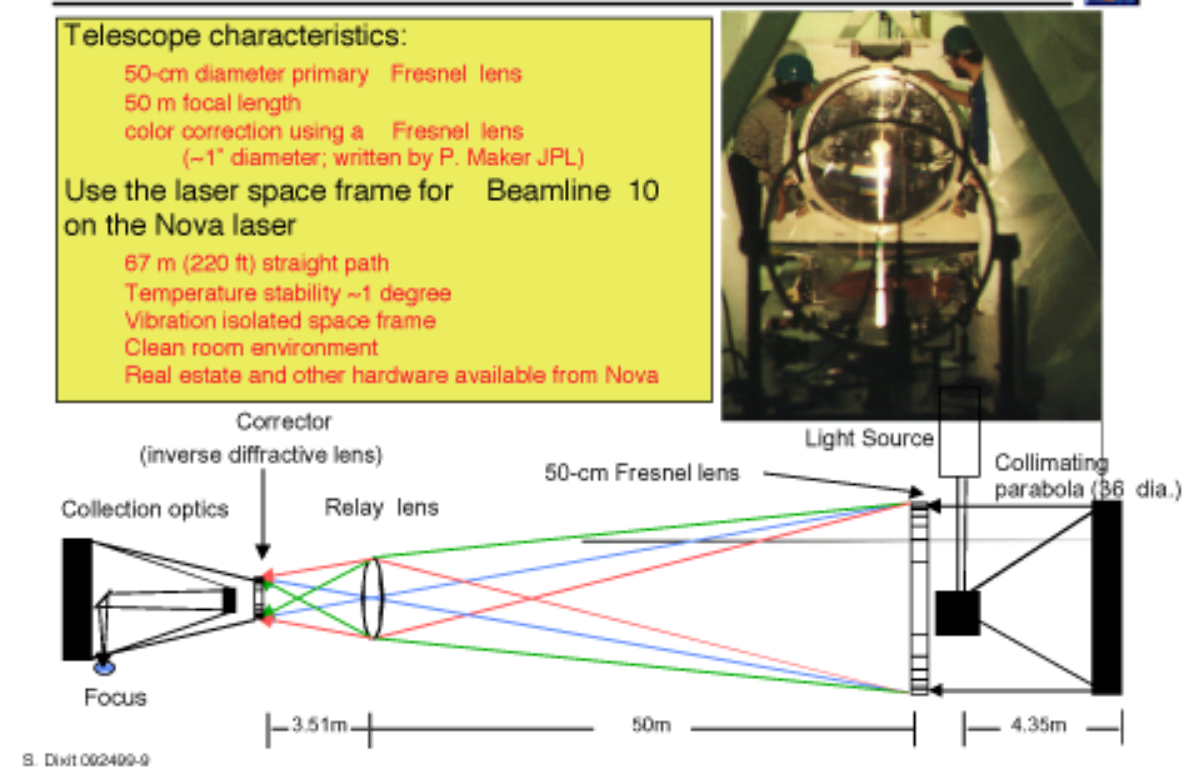


We have demonstrated near-diffraction limited imaging on the $50-\mathrm{cm}$ diffractive telescope in the Nova laser bay

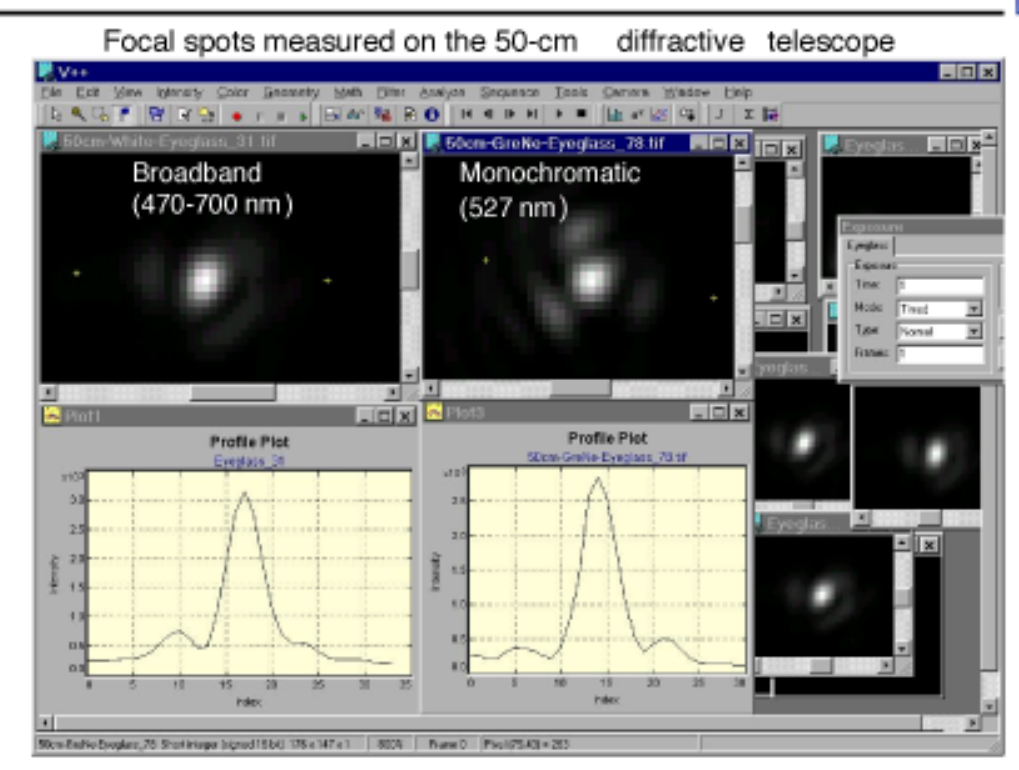

\section{Summary}

- discussed how diffractive telescopes offer a powerful alternative way for broad-band earth and space observation

require large Fresnel lens on thin membrane as the primary can be fully color corrected

- demonstrated diffraction limited performance for the $20-\mathrm{cm}$ and $50-\mathrm{cm}$ telescopes

- obtained astronomical images using the 20-cm telescope 


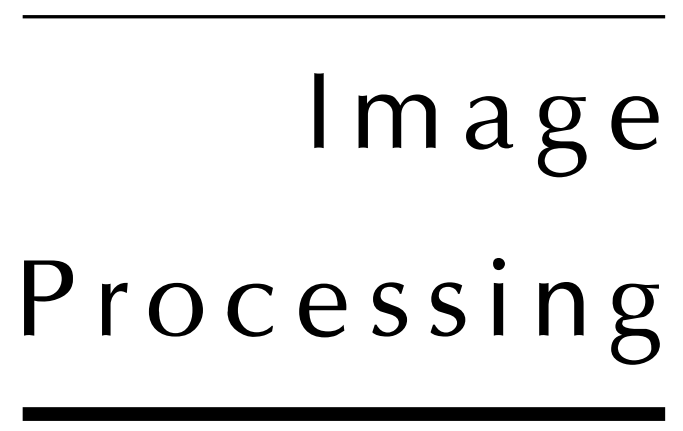




\title{
Detection of Vertical Obstructions in SAR Images
}

\author{
UCRL-VG-136063 \\ Sailes K. Sengupta and Ursula Goldstein \\ Paper submitted to the Signal and Imaging Sciences Workshop \\ November 11-12, 1999 \\ Abstract
}

Finding vertical obstructions such as towers, buildings, hanging wires, etc. is an important part of generating up-to-date maps or related spatial databases. SAR imagery can be a useful source of information in this process because cloud cover does not interfere with imaging and because many of these objects produce strong radar returns. The problem of automatically detecting vertical obstructions has been addressed in this work using methods derived from pattern recognition theory and morphological image processing. Hanging wires approximated by low curvature parabolas have been detected in this work by a modified version of the Randomized Hough Transform (RHT). Towers, buildings and other 'bright' or 'dark' objects have been detected by clustering pixels in a feature space consisting of neighborhood statistics of pixels. Towers are localized from among the bright objects using their shape features. Possible locations of buildings have been detected using the morphological features of their shadows obtained from clustering. Finally, the need for the creation of an appropriate perceptual information framework has been indicated in situations where a successful integration of information from different sensors and existing models is essential.

Work performed under the auspices of the U.S. Department of Energy by Lawrence Livermore National Laboratory under Contract W-7405-ENG-48. 


\section{Use of Morphological Operators and Pattern Recognition Techniques for Sorting the FIRST Data}

\section{Deanne Proctor}

The application of morphological and pattern recognition techniques to the sorting of FIRST data is presented. FIRST (Faint Images of the Radio Sky at Twenty Centimeters) is designed to produce the radio equivalent of the Palomar Observatory Sky Survey, using the National Radio Astronomical Observatory Very Large Array. A catalog of source positions and Gaussian-fit parameters for the sources is available.

The goal of this part of the project is the automatic classification of types of sources, since it is expected that there will be on the order of one million sources in the final catalog.

The data consists of radio maps, each approximately $1500 \times 1100$ pixels and containing on average about 30 sources per map. The resolution of these maps is 5" (approximately 3 pixels). The sources range in size from a few pixels to 70 or 80 pixels maximum diameter and have various morphologies. Of particular interest is the extraction of 'bent doubles', core jets, and gravitational lens candidates. These interesting types constitute on the order of percent of the sources.

The emphasis in this presentation is on image-based pattern recognition. We report on the use of morphological operators for source extraction and compare use of decision trees and neural networks in the classification of source morphologies and accounting for the finite resolution.

Keywords: pattern recognition, morphology 


\title{
USE OF MORPHOLOGICAL OPERATORS AND \\ PATTERN RECOGNITION TECHNIQUES FOR SORTING THE FIRST DATA
}

\author{
Deanne Proctor \\ Scientific Computing Applications, Computations \\ Lawrence Livermore National Laboratory
}

November, 1999.

\section{RESOURCES}

- FIRST maps

(FIRST survey, Robert Becker, P.I.)

- IDL Interactive Data Language (Research Systems, Inc.)

- IDL UIT database software (Landsman)

- IDL FIRST catalog in database form (Michael Gregg)

- OC1 decision tree software (Murthy, Salzberk, Kasif, Johns Hopkins Univ.)

- SNNS Stuttgart Neural Network Simulator (Institute for Parallel and Distributed High Performance Systems, Univ. of Stuttgart)

Thanks to IGPP, Charles Alcock, Kem Cook for office and computing facilities. 


\section{OUTLINE}

\section{INTRODUCTION}

II. SOURCE EXTRACTION
A. Catalog
B. Morphological Operators
C. Groupings

III. PATTERN RECOGNITION
A. Invariance
B. Training and Test Sets
C. Feature Selection
D. Decision Trees and Neural Networks

IV. RESULTS

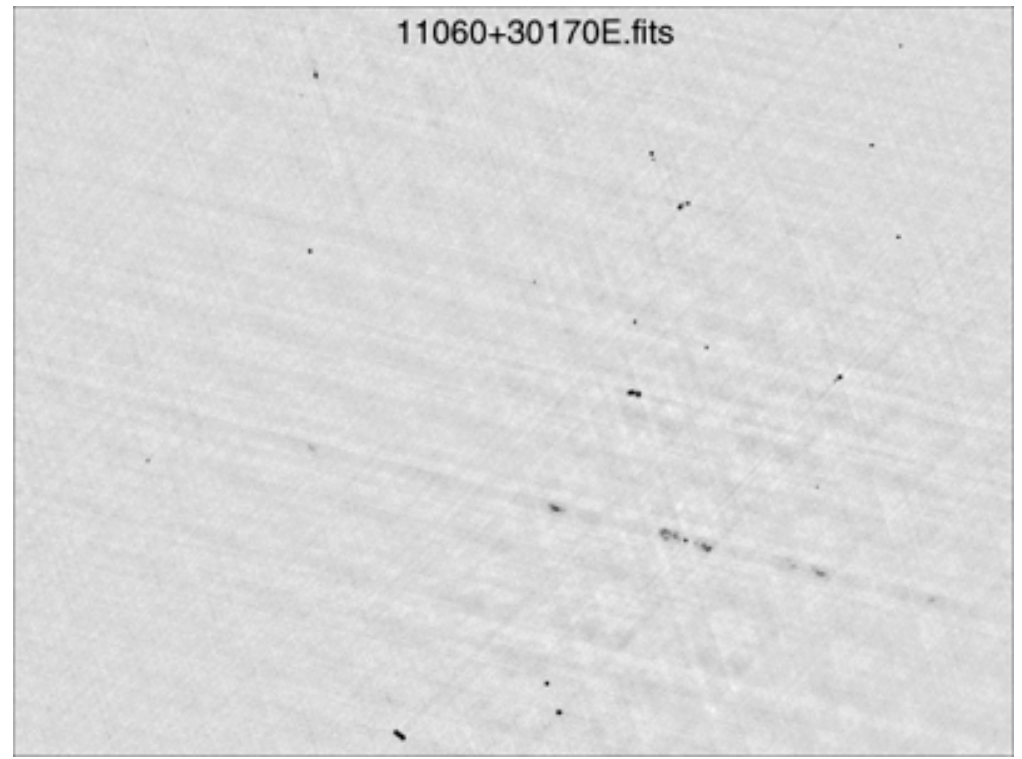




\section{SOURCE EXTRACTION}

- catalog

Up to 4 Gaussians fit per source island.

Software to group catalog sources by proximity.

$\begin{array}{lr}\text { Singles } & 71 \% \\ \text { Doubles } & 18 \% \\ \text { Triples } & 6 \% \\ \text { Four and more } & 5 \%\end{array}$

- MORPHOLOGICAL

Erode, Dilate and Label Region

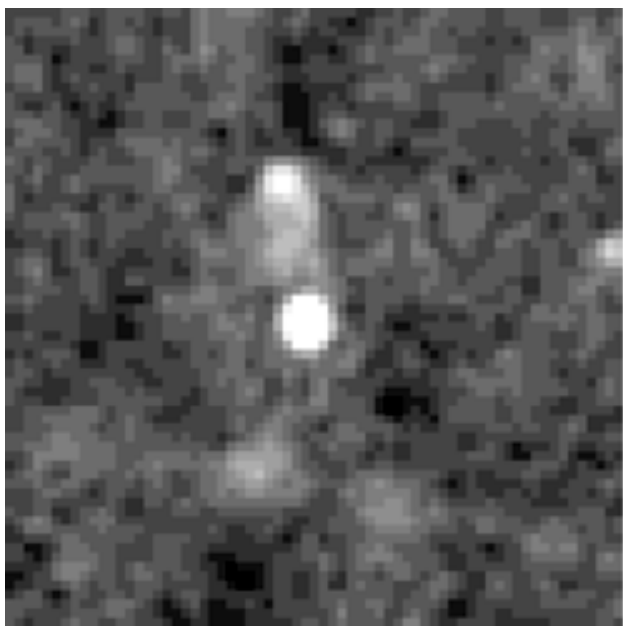

n98_109888.first.fits

image 
I M A G E P R O C E S S I N G

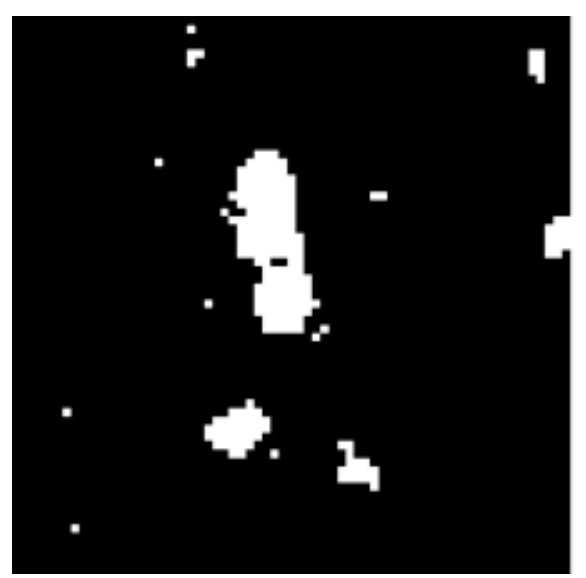

n98_109888.first.fits

3 sigma threshold of image

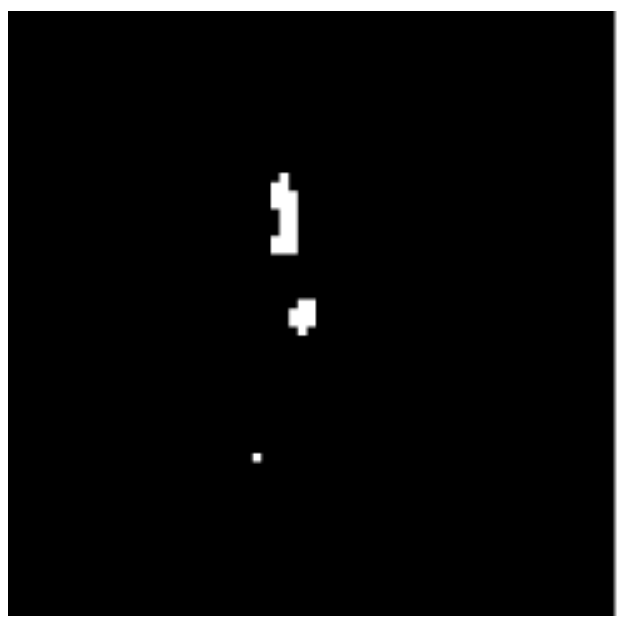

n98_109888.first.fits

erode(image_thr3,s5) 


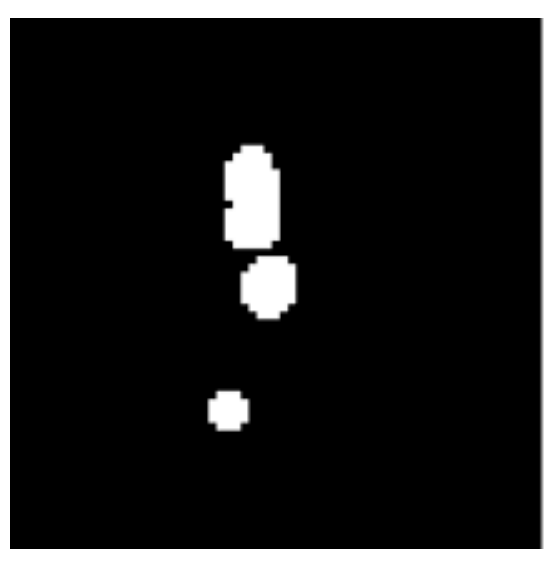

n98_109888.first.fits

dilate(erode(image_thr3,s5),s5)

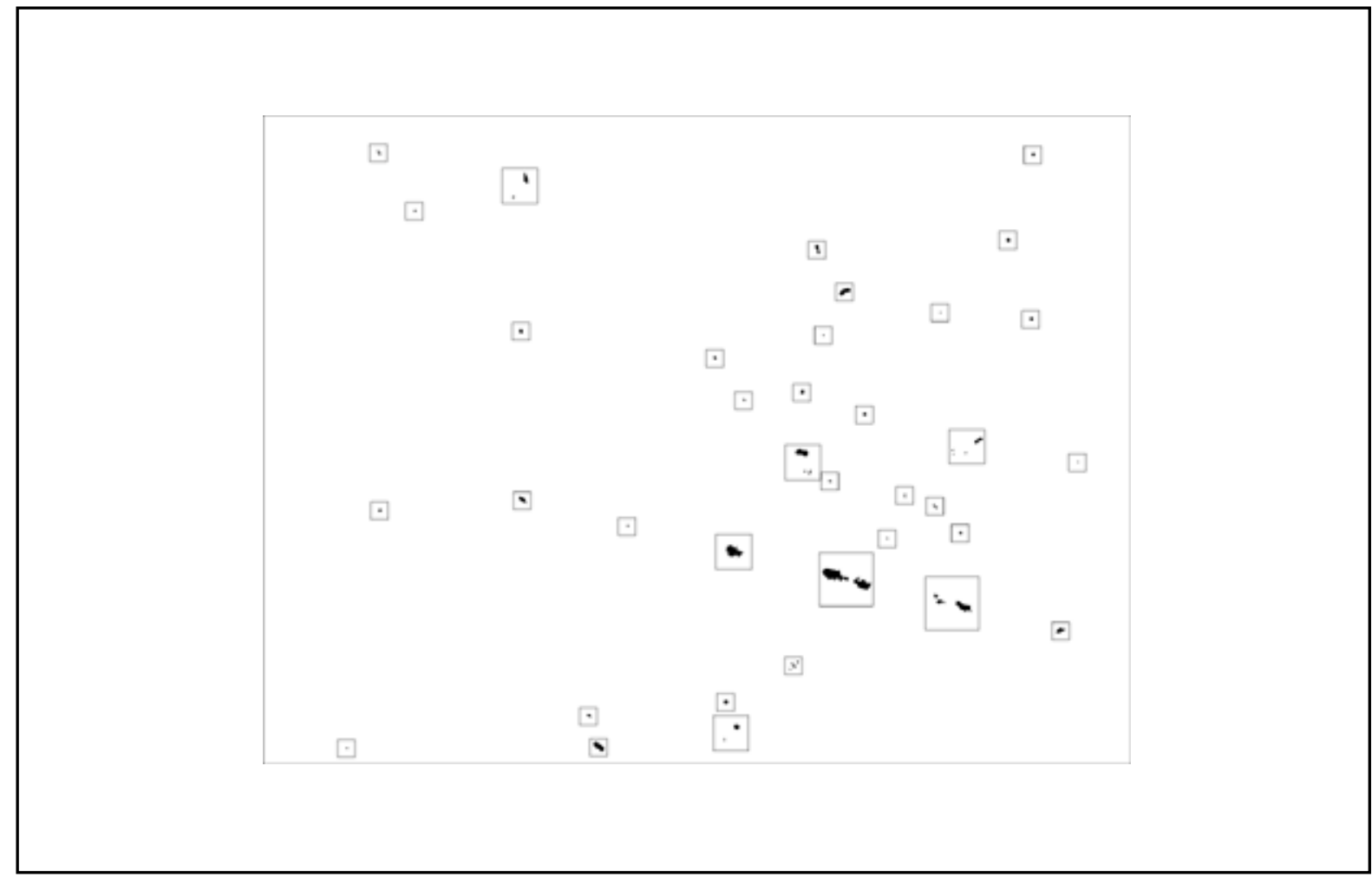


I M A G E P R O C E S S I N G

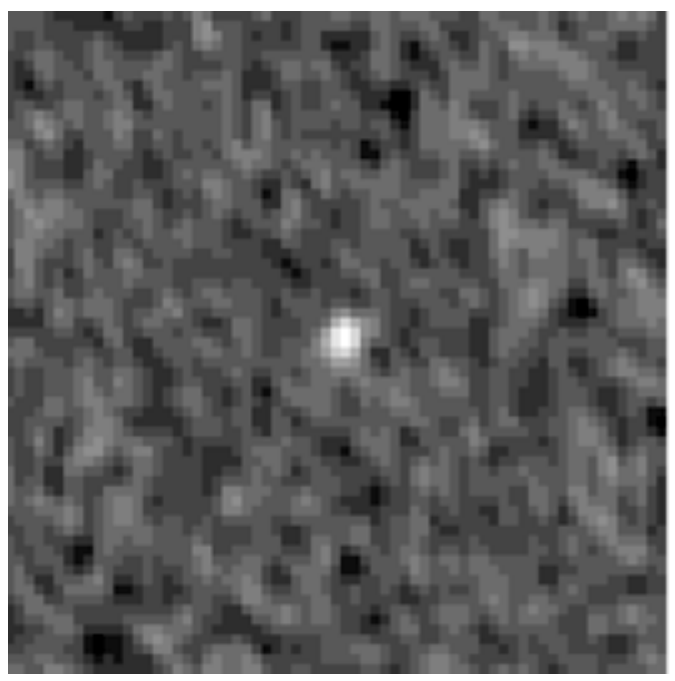

s98_000744.fits

SINGLE

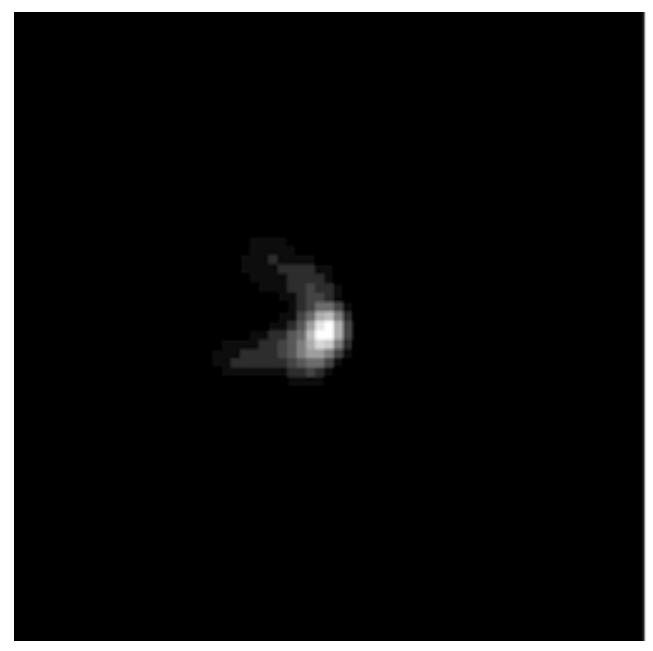

s98_00210.first.fits

SINGLE 


\section{M A G E P R O C E S S I N G}

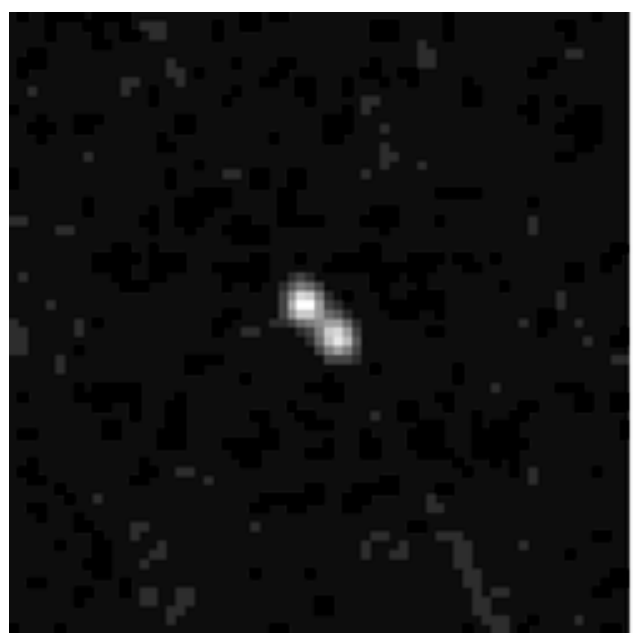

north98_074343.fits

DOUBLE

north98_076235.fits

DOUBLE 
I M A G E P R O C E S S I N G

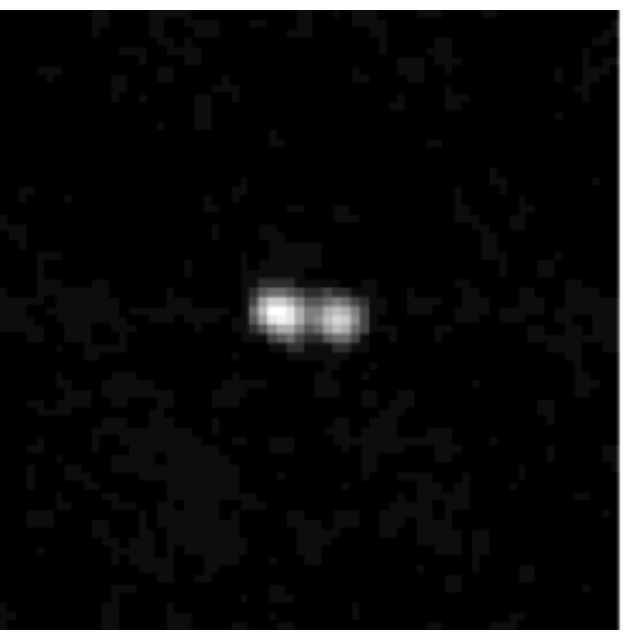

north98_103126.fits

DOUBLE

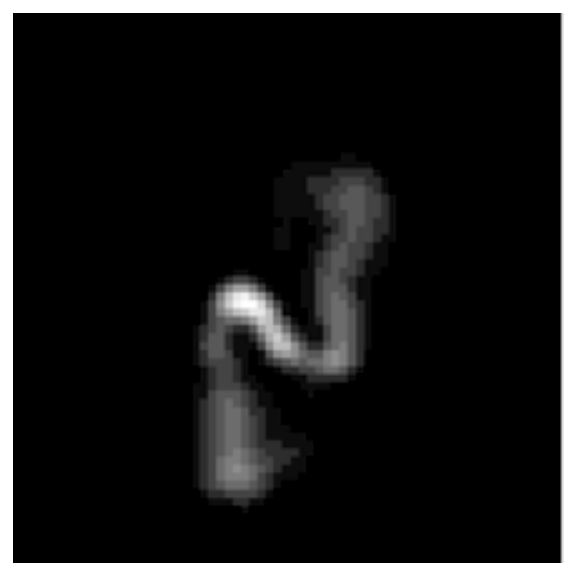

north98_069940_069971.fits

SPIRAL 


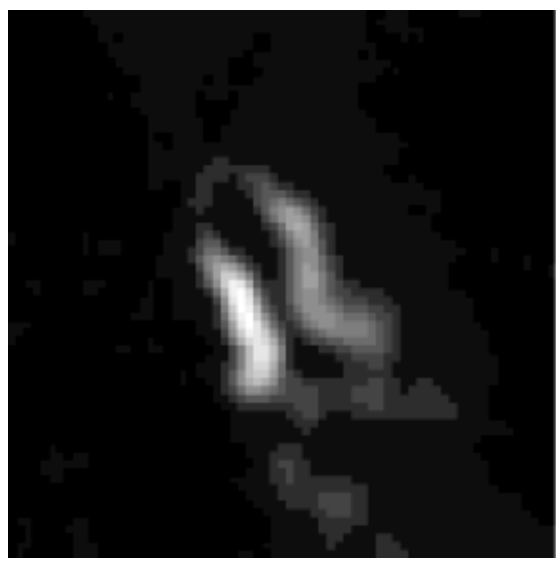

South98_008442_008447.fits

NAT

\section{CLASSICAL INVARIANCES REQUIRED}

- Translation

- Rotation

- Scale - this is the problem, due to finite point spread function 
I M A G E P R O C E S S I N G

TRAINING SET - Visual Classifications

233 Bent

426 Somewhat Bent

1255 Non-Bent

721 ? (too noisy to tell)

10 Misc.

2645 Total

TEST SET - Visual Classifications

52 Bent

165 Somewhat Bent

612 Non-Bent

227 ? (too noisy to tell)

3 Misc.

1059 Total

\begin{tabular}{|c|c|c|}
\hline METHOD & BENTS FOUND (\%) & FALSE POSITIVE (\%) \\
\hline OC1 decision tree & 71 & 75 \\
\&catalog features & & \\
OC1 decision tree & & \\
\&image statistics & & \\
SNNS Rprop & & \\
\hline
\end{tabular}


I M A G E P R O C E S S I N G
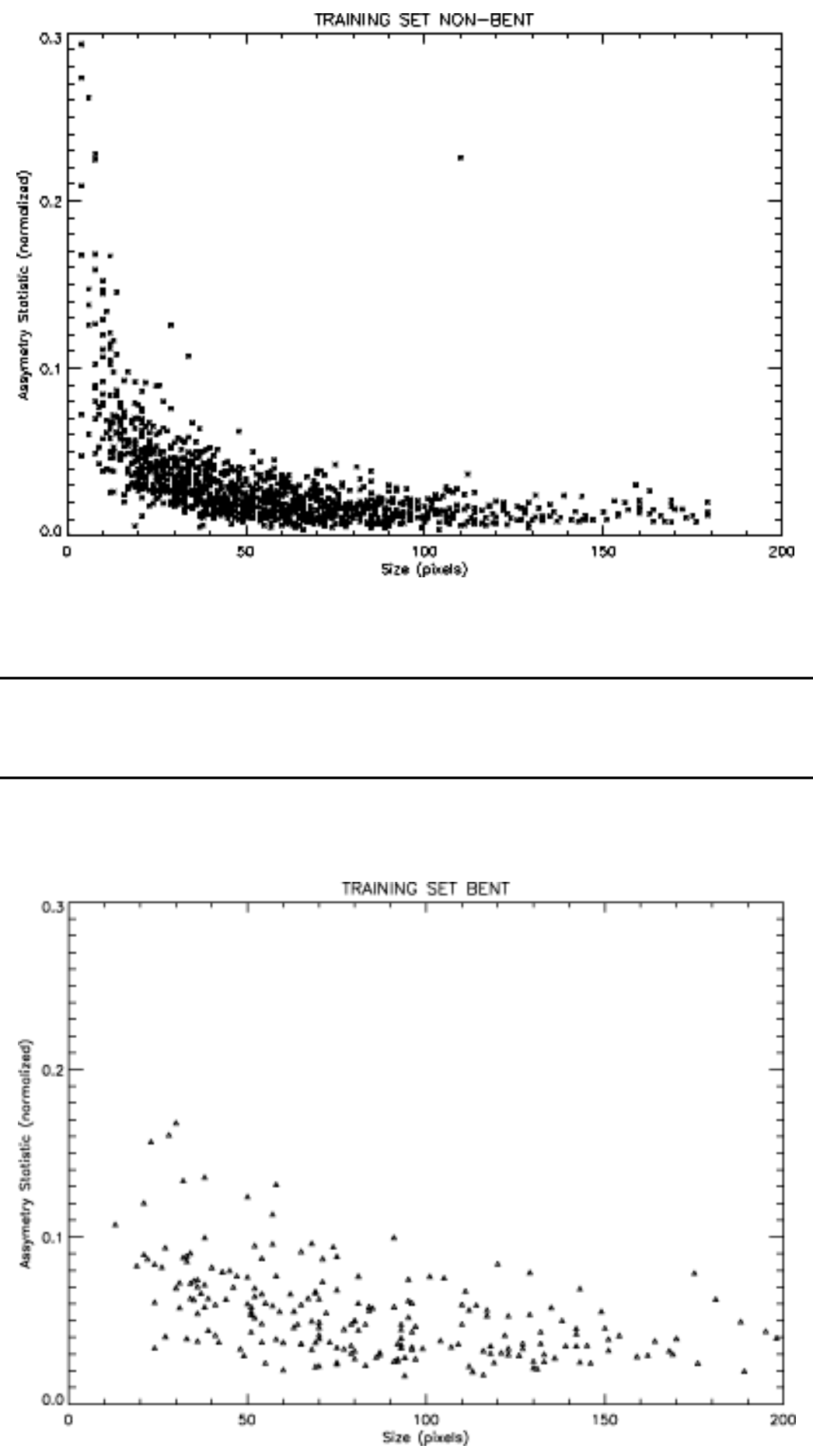
$\begin{array}{lllllllllllllll}\text { I } & M & A & G & E & P & R & O & C & E & S & S & \text { I } & N & G\end{array}$

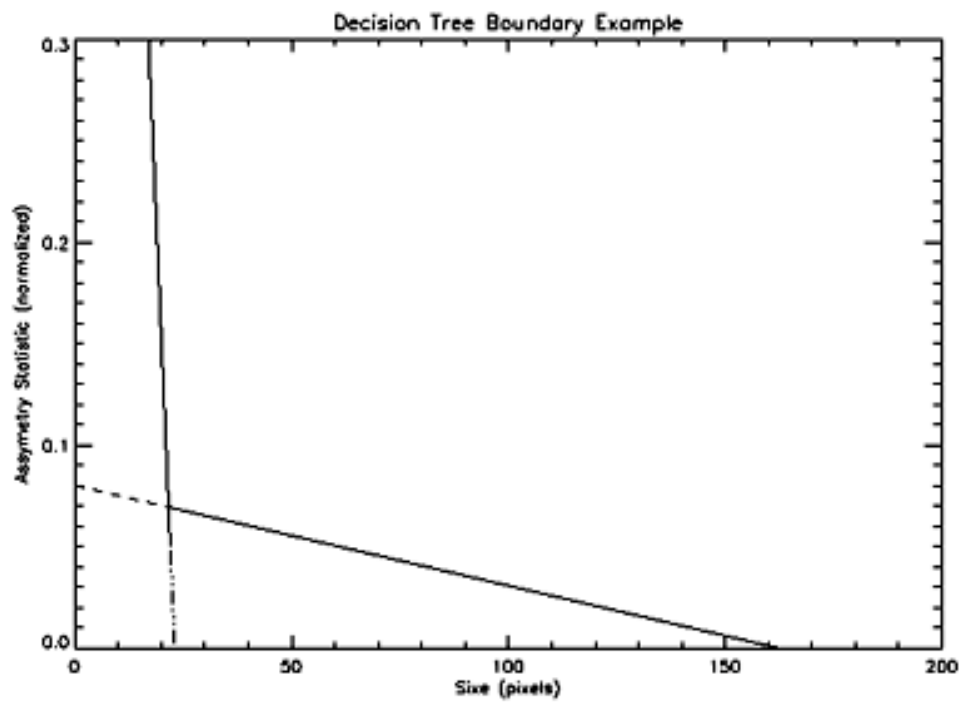

\begin{tabular}{|c|c|c|}
\hline METHOD & BENTS FOUND (\%) & FALSE POSITIVE (\%) \\
\hline $\begin{array}{c}\text { OC1 decision tree } \\
\text { \&catalog features }\end{array}$ & 71 & 75 \\
$\begin{array}{c}\text { OC1 decision tree } \\
\text { \&image statistics } \\
\text { SNNS Rprop }\end{array}$ & 88 & 54 \\
\hline
\end{tabular}




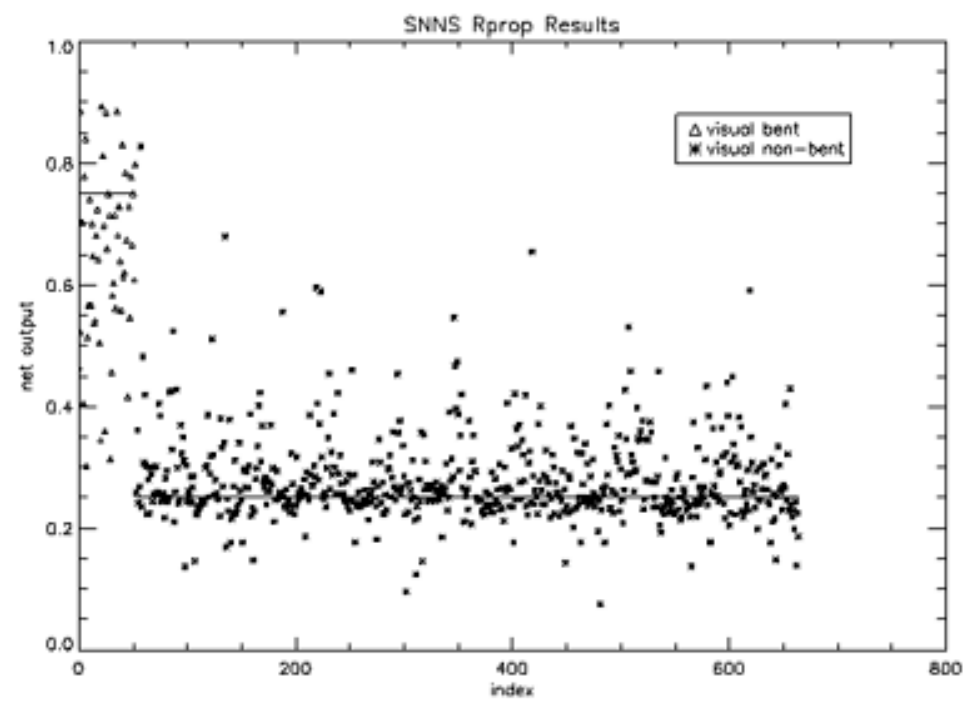

\begin{tabular}{|c|c|c|}
\hline METHOD & BENTS FOUND (\%) & FALSE POSITIVE (\%) \\
\hline OC1 decision tree & 71 & 75 \\
$\begin{array}{c}\text { \&catalog features } \\
\text { OC1 decision tree }\end{array}$ & 88 & 54 \\
$\begin{array}{c}\text { \&image statistics } \\
\text { SNNS Rprop }\end{array}$ & 85 & 25 \\
\hline
\end{tabular}


I M A G E $\quad$ M $R$ O O C C E S S I N G

Visual Classifications of 115 OC1 Selected

Non-axisymmetric Sources:

$$
\begin{aligned}
& 27 \text { Yes (NAT \& WAT) } \\
& 21 \text { Maybe } \\
& 15 \text { Core-Jet } \\
& 52 \text { No (Spiral \& Other) }
\end{aligned}
$$

The future work is to automate these classifications. 


\title{
Noninvasive Recovery of Local Properties of Deforming Objects from Registered Range Data
}

\author{
UCRL-JC-135878ABS
}

Leonid V. Tsap(1), Dmitry B. Goldgof(2) and Sudeep Sarkar(2)

(1) Lawrence Livermore National Laboratory

Center for Applied Scientific Computing
(2) Department of Computer Science and Engineering University of South Florida

Physically-based modeling involves the use of natural properties of nonrigid objects to determine motion parameters. What if some local material properties or geometry are unknown? Generalization and simplification attempts compromise the advantages gained from using such models. In this talk I present a noninvasive approach for iterative recovery of such initially unknown parameters. The method is based on the analysis of the differences between the actual behavior of the object (recovered from registered range and intensity image sequences) and its predicted behavior using a model. Large differences indicate that an object's properties are not captured properly by the model describing it. Feedback from the images during the motion allows for the refinement of the model by minimizing the error between the expected and true position of the object's points. Unknown parameters are recovered using an iterative descent search for the best nonlinear finite element model that approximates observed motion. As a result, we obtain a more precise description of the object including initially unknown local material properties or geometry.

Experimental results demonstrate the success of the proposed algorithm. The method was applied to man-made elastic materials and human skin to recover unknown elasticity, to complex 3-D objects to find details of their geometry, and to a hand motion analysis application. A very important application of the method is that it is capable of objective detection of the differences in elasticity between different materials as well as measuring burn scar elasticity and including it into the next, higher level of modeling such as our model of the hand. The latter is used for an assessment of the feasibility of applying combined computer vision and simulation techniques to the analysis of Repetitive Stress Injury (RSI). Experiments also show method's capabilities to detect the problems with location or size of specific parts of deformable objects. This work demonstrates the possibility of accurate quantitative analysis of nonrigid motion in range image sequences with objects consisting of multiple materials and 3-D volumes.

Keywords: physically-based vision, deformable models, nonrigid motion analysis, biomedical applications

This research was supported in part by the Whitaker Foundation Biomedical Engineering Research Grant and in part by the National Science Foundation Grants IRI-9619240 and EIA-9729904.

This work was performed under the auspices of the U.S. Department of Energy by Lawrence Livermore National Laboratory under contract number W-7405-Eng-48. 


\section{Noninvasive recovery of local properties of}

deforming objects from registered range data

\section{Leonid V. Tsap (1), Dmitry B. Goldgof (2) and Sudeep \\ Sarkar (2)}

(1) Lawrence Livermore National Laboratory

Center for Applied Scientific Computing

(2) Department of Computer Science and Engineering

University of South Florida

November 11, 1999

\section{Motivation}

Physically-based modeling in vision and its requirements

I The need to achieve precise motion analysis and object understanding given incomplete description of the object

I The method uses:

input: sequences of registered intensity and range images

output: displacement fields, strain distributions and recovered geometry/ material properties

Applicational requirements in burn scar assessment, repetitive stress injury analysis and local shape estimation of deformable objects 


\section{Main steps of the method}

Establish corresponding features from images

Detect areas with unknown properties: create physically-based model compute motion (by applying displacements) analyze strain distribution detect abnormal areas

Recover properties:

change affected property for such areas compute model after the motion estimate the error (differences with image data) continue until minimum

\section{Experimental setup}

The structured light system used in this research projects a sequence of horizontal stripes into the scene

I The image is captured from a different viewpoint with a camera
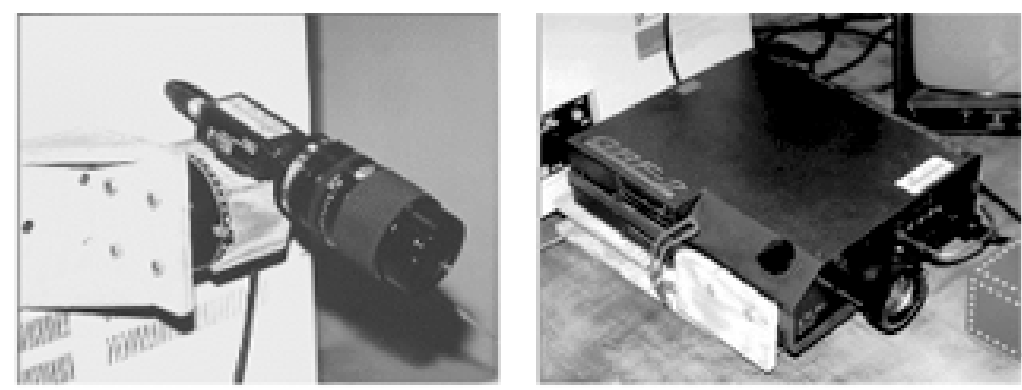


\section{Data acquired by the system}

Intensity images of the elastic material before and after stretching. The grid painted on the bandage is used for tracking.
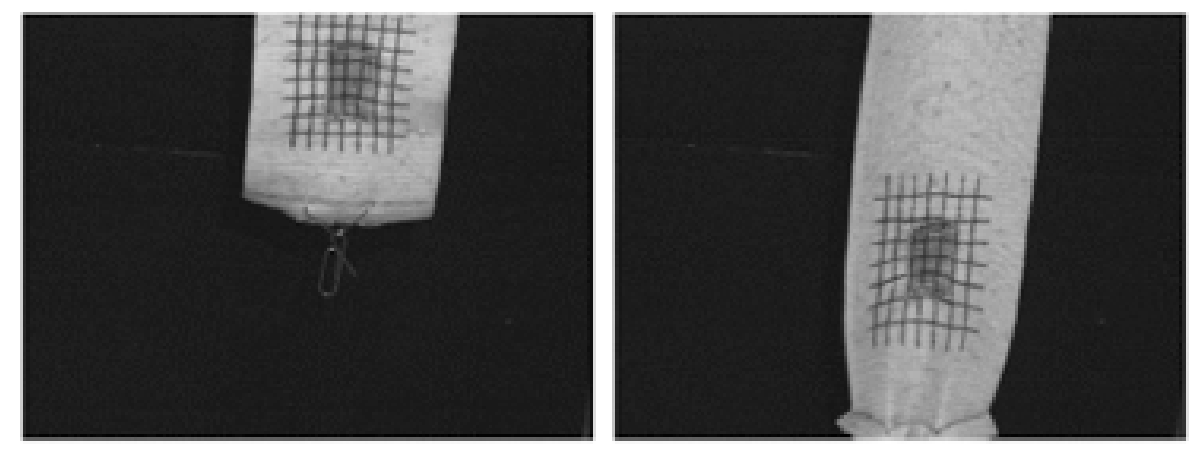

\section{Data acquired by the system}

Range images of the elastic material before and after stretching
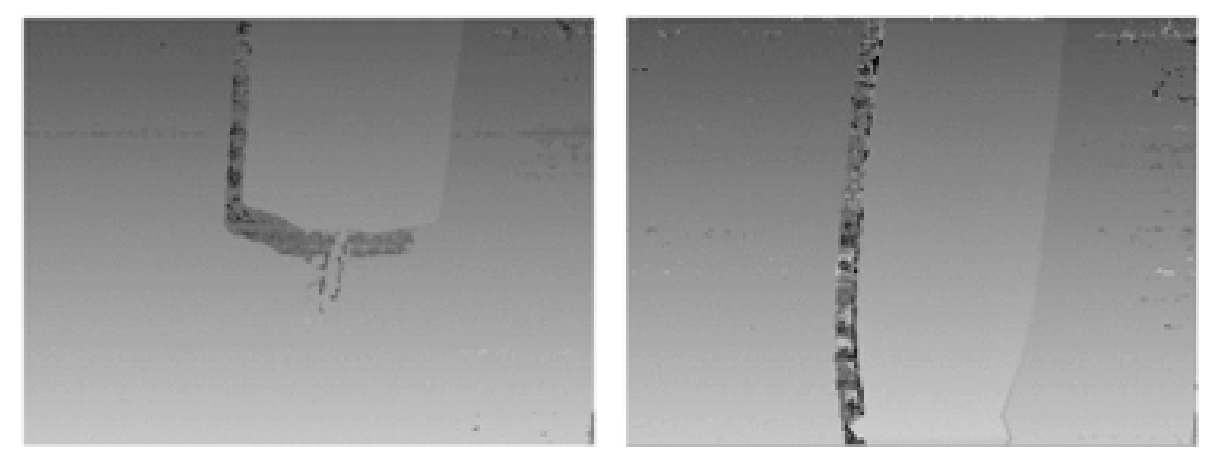


\section{Application of Active Contours}

A snake is an energy minimizing spline, governed by internal and external forces. It is specified by a set of control points.

The energy function for a snake:

$$
E_{\text {snake }}=E_{\text {internal }}+E_{\text {external }}
$$

Total snake energe definition for a greedy algorithm by Williams and Shah:

Òa (s) $\left.E_{\text {cont }}+\mathrm{b}(\mathrm{s}) E_{\text {curv }}+\mathrm{g}(\mathrm{s}) E_{\text {img }}(\mathrm{s})\right) d s$

Snake placement

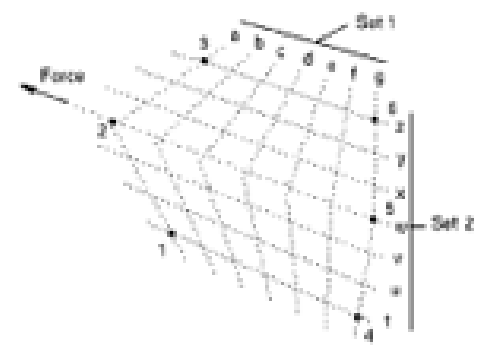

\section{Recovering material properties}

Applying snakes to the intensity images (control points are red, snake intersections are yellow)
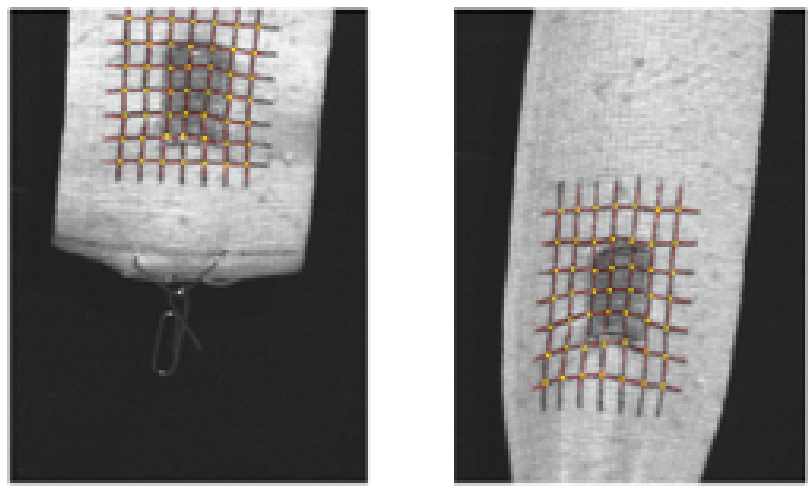

Detected intersections are used to build a finite element model 


\section{Recovering material properties}

Detection of abnormal area, pinpointed by the lowest strain (blue)

Iterative descent search for its properties (selected iterations)
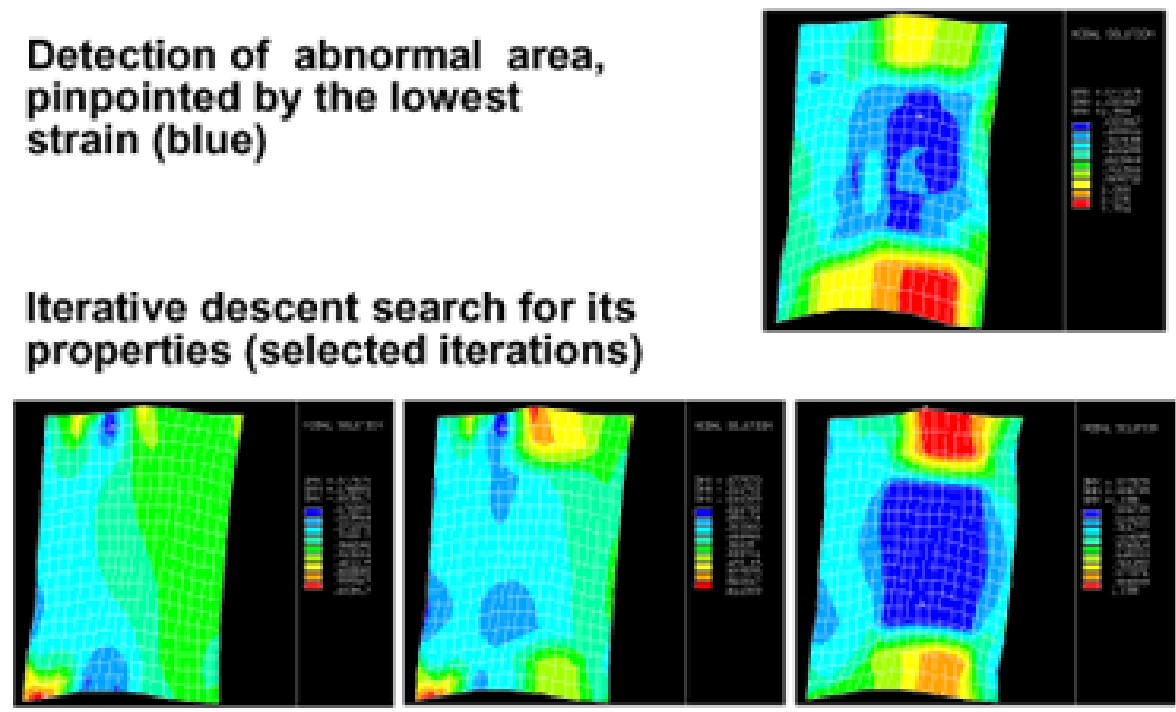

\section{Recovering material properties}

Approximation of the error function (differences in displacements between the model and the object during the search). Minimum of the function corresponds to recovered elasticity of the abnormal area.

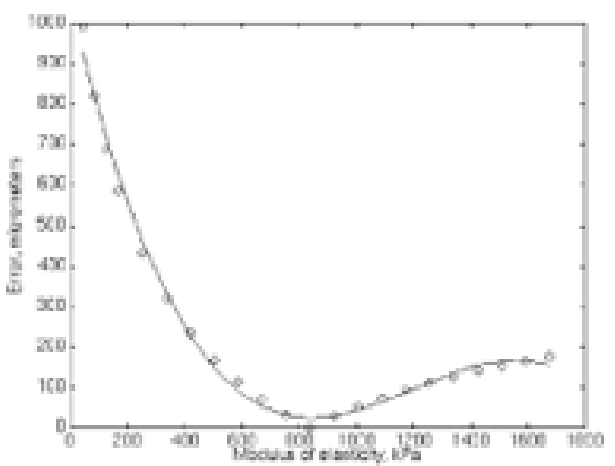




\section{Objective assessment of burn scars}

I Detection of scars from images

I Evaluation of their elastic properties relative to the surrounding tissues

I Ranking scars on the scale used by physicians

| Providing an objective criteria for healing progress estimation and comparison of techniques

Similarly to elastic materials, distortions are evaluated when skin is pulled

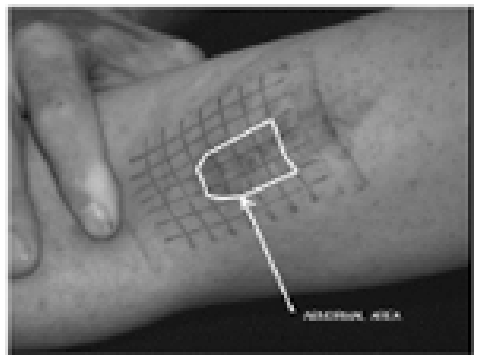

\section{Objective assessment of burn scars}
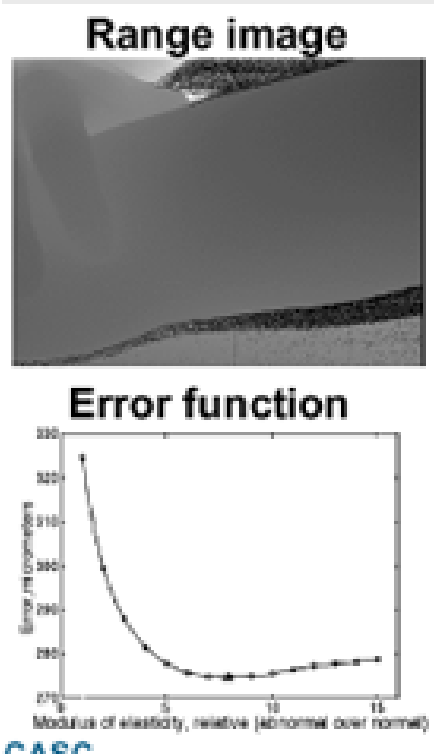

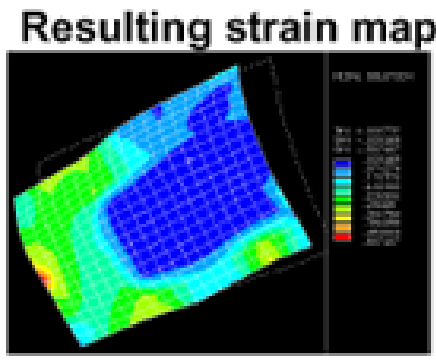

Scar rating by the specialist

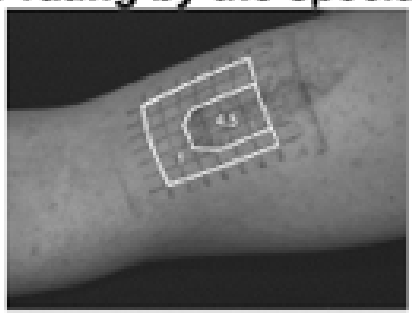




\section{Recovering missing geometry information}

I Often some info about local geometry is not available

I Consider a cup holder sequence (range not shown)
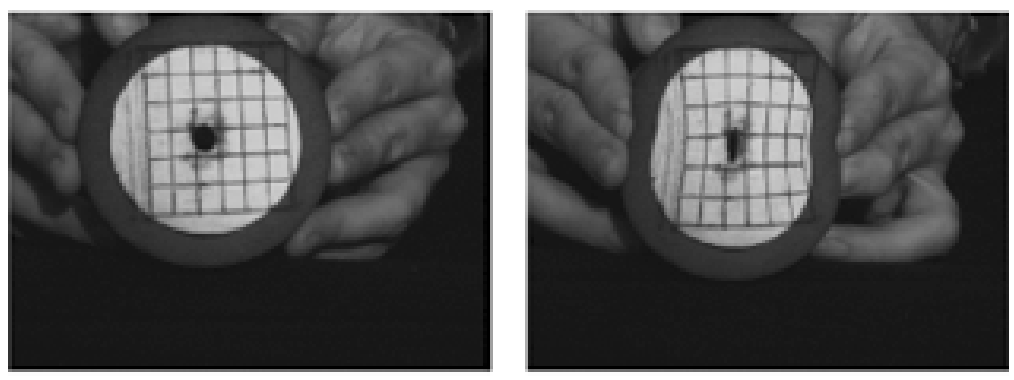

\section{Recovering missing geometry information}

I Area of interest is characterized by high strains (red)

I Missing info (hole in the bottom of the cup holder) is recovered with a local model and integrated into a 3-D model
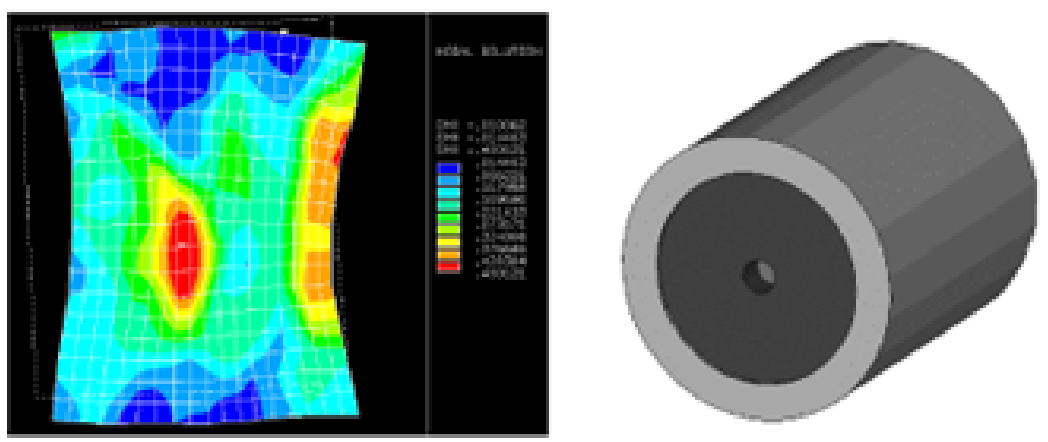


\section{Hand motion reconstruction and integration of recovered properties}

I Various positioning of the hands on the keyboard

I Wide range of movements

I Strains are projected onto the surface

| Looking for excessive strains

I Influence of burn scars on the hand motion (by including recovered properties)
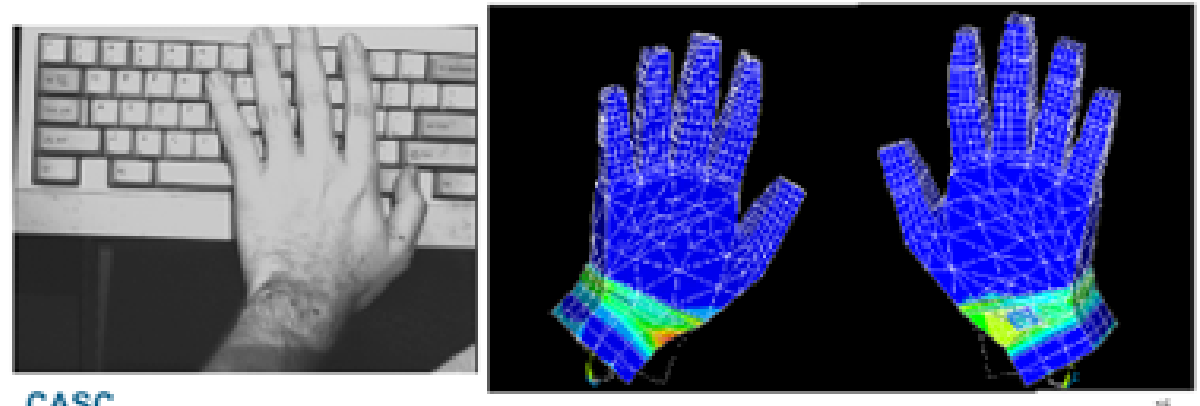

\section{UCRL-VG-135878}

Work pertormed under the auspices of the U. S. Department of Energy by Lawrence Livermore National Laboratory under Contract W-740S-Eng-43 


\section{Subsurface Gap Depth Detection by Infrared Imaging Using a Surface Heat Pulse}

Charles Landram, Richard Martin, Nancy DelGrande and Philip Durbin 


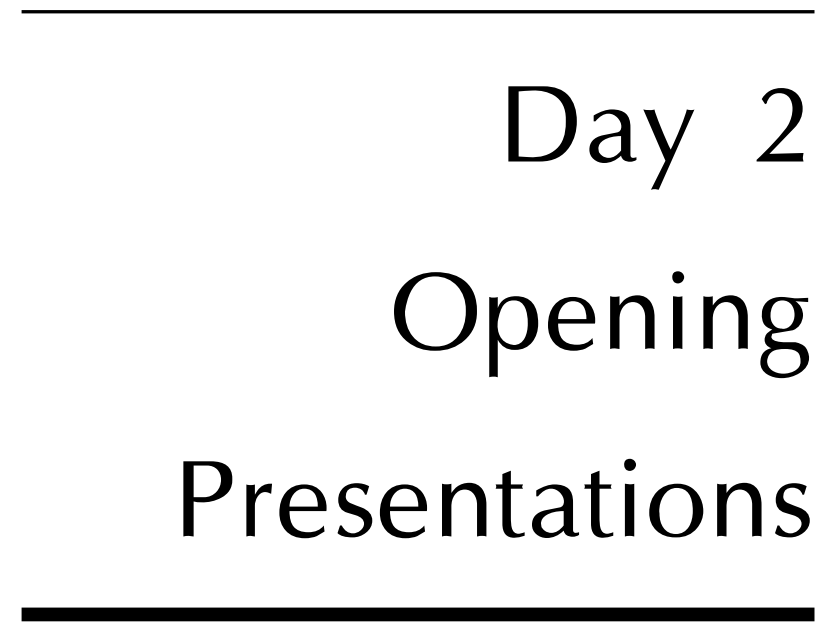



D A Y
2 :
O P E N I N G
$\begin{array}{lllllllllllll}P & R & E & S & E & N & T & A & T & \text { I } & \text { O } & N & S\end{array}$

\title{
Engineering Research and Development
}

\author{
Spiros Dimolitsas
}

ENGINEERING

\section{Engineering's Competencies}

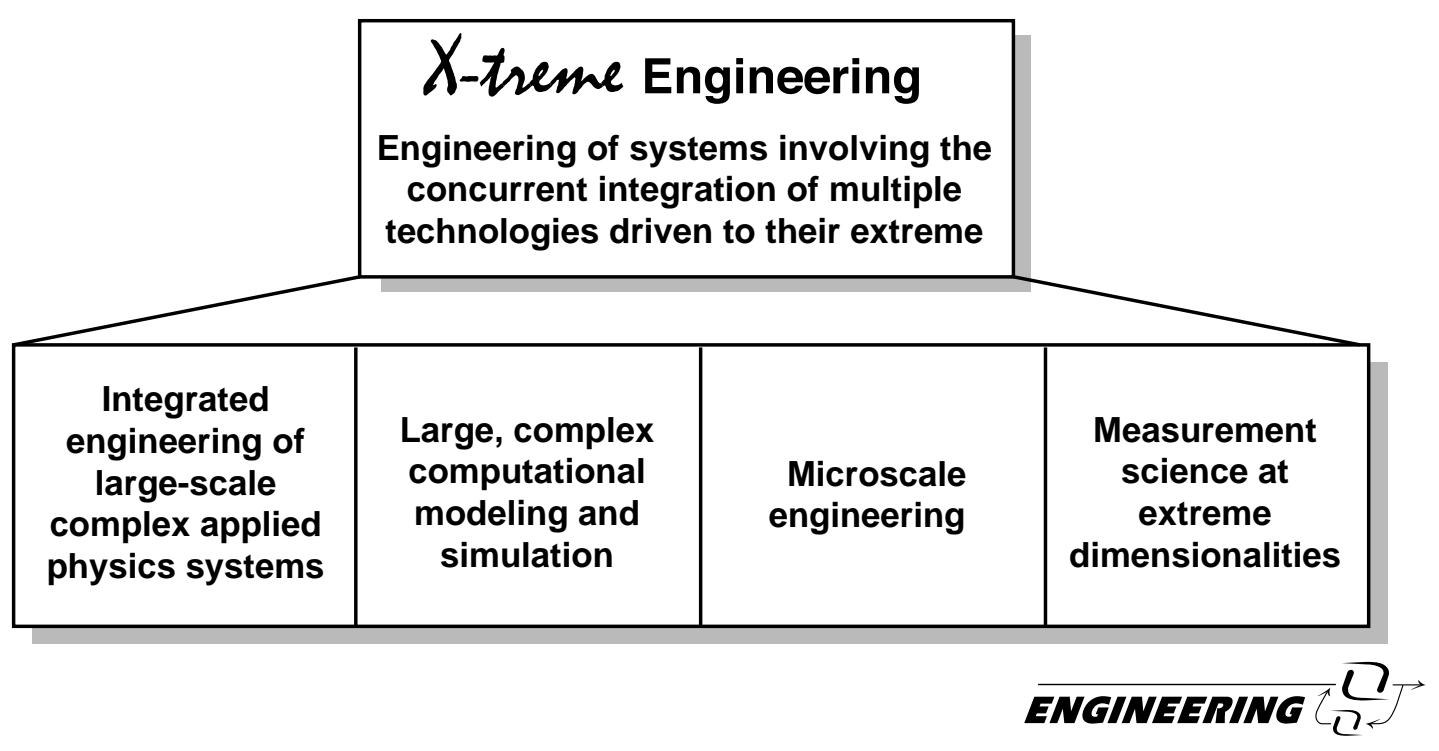


$\begin{array}{llllllllllllllllllllllllll}D & A & Y & 2 & : & O & P & E & N & \text { I } & N & G & P & R & E & S & E & N & T & A & T & I & O & N & S\end{array}$

\section{Engineering's Core Technologies}

- Systems Engineering

- Computational Engineering

- Precision Engineering

- Microsystems Technology

- Signal Acquisition and Characterization

ENGINEERING

Engineering's Structure Reflects its Technologies

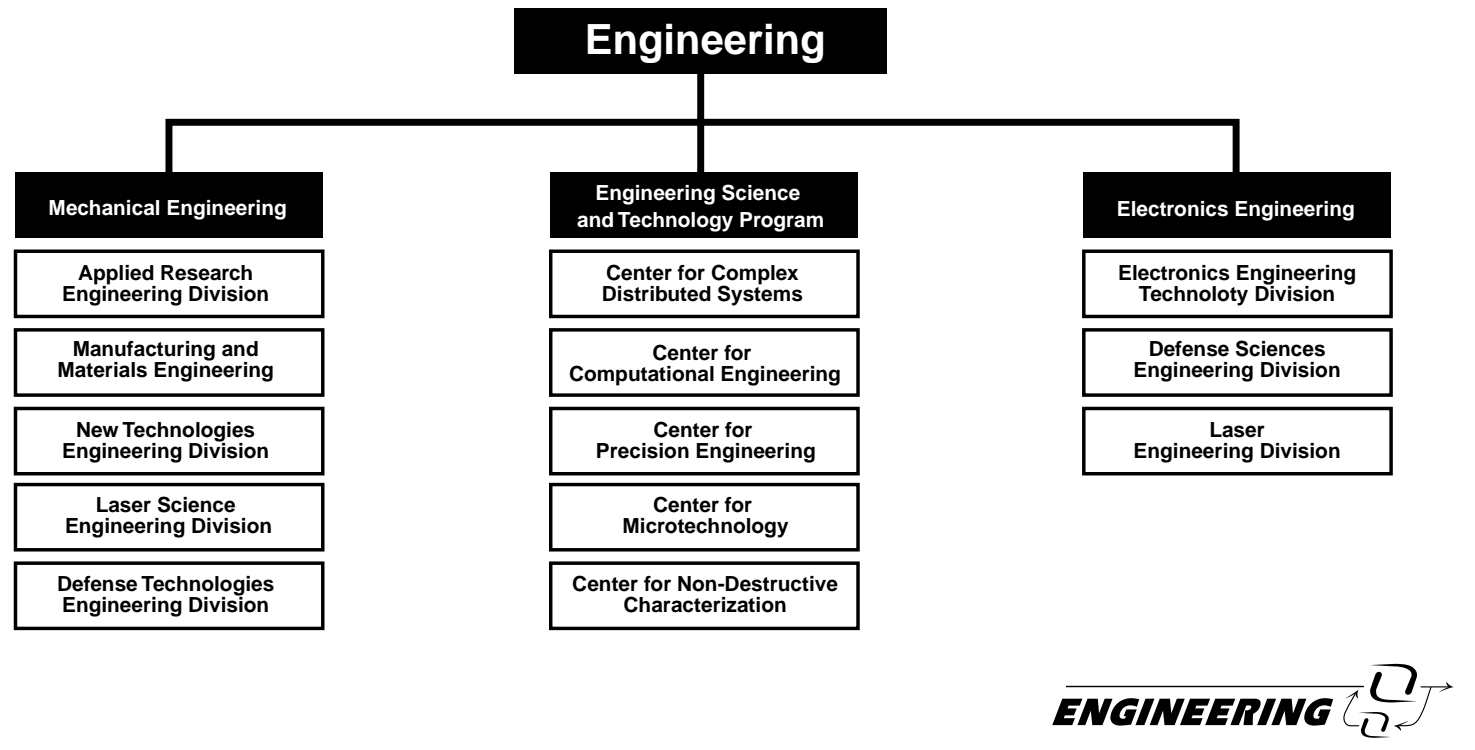



D A Y
$2:$
O P E N I N G
P R R E S

\section{Engineering Center Mission}

- Engineering's Technology Centers are responsible for the vitality and growth of the core technology each of them represent:

- They focus and guide Engineering's investments in enabling technologies and supporting capabilities

- Each Center is an Engineering R\&D "program" whose output is technology

- Centers provide a forum for the discussion of issues related to the broader core technology area with which each of them is associated

\section{Each Center addresses a number of enabling technologies within their sphere of activity}

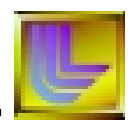

\begin{tabular}{c|c|c|c|c}
$\begin{array}{c}\text { Computational } \\
\text { Engineering }\end{array}$ & $\begin{array}{c}\text { Precision } \\
\text { Engineering }\end{array}$ & $\begin{array}{c}\text { Micro- } \\
\text { Technology }\end{array}$ & $\begin{array}{c}\text { Non-Destructive } \\
\text { Characterization }\end{array}$ & $\begin{array}{c}\text { Complex } \\
\text { Distributed } \\
\text { Systems }\end{array}$ \\
$\begin{array}{c}\text { Integrated } \\
\text { modeling of } \\
\text { assembled } \\
\text { engineering } \\
\text { systems }\end{array}$ & $\begin{array}{c}\text { Removal, } \\
\text { addition, and } \\
\text { measurement of } \\
\text { material at near- } \\
\text { atomic level }\end{array}$ & $\begin{array}{c}\text { Microscale } \\
\text { fabrication of } \\
\text { fluidic and } \\
\text { photonic } \\
\text { structures and } \\
\text { systems }\end{array}$ & $\begin{array}{c}\text { Quantitative } \\
\text { non-destructive } \\
\text { characterization } \\
\text { of components } \\
\text { and systems at } \\
\text { high resolution }\end{array}$ & $\begin{array}{c}\text { Simulation, } \\
\text { control and } \\
\text { sensing of large- }\end{array}$ \\
& & systems
\end{tabular}



D A Y
2 :
O P E N I N G
P R E S E N T A T I O N S

\section{Engineering's Core Technologies}

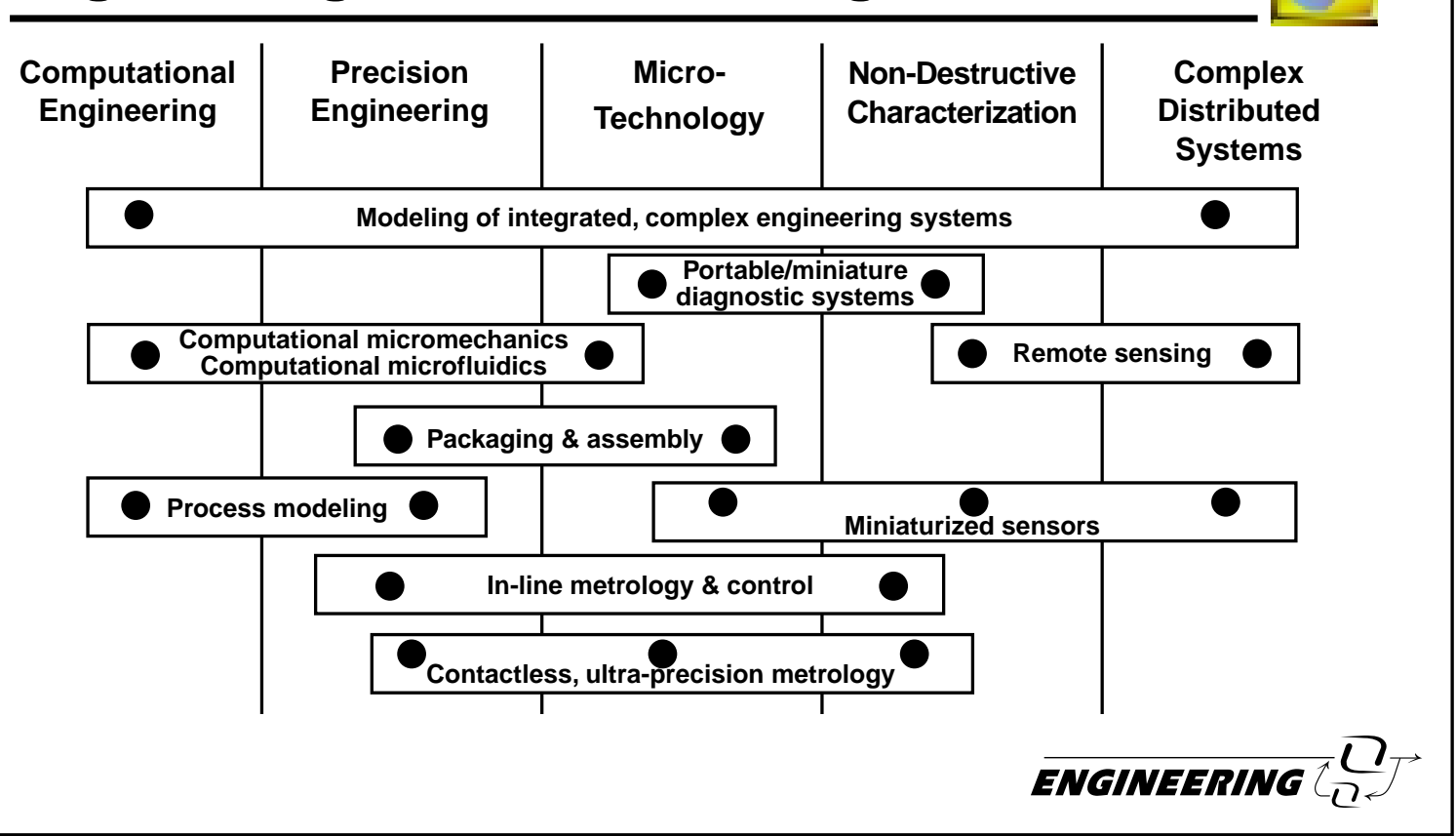




\title{
A Retrospective on Computer Vision Research
}

\author{
Avi Kak, Professor in the School of Electrical Engineering, Purdue University
}

During the last thirty years, computer vision has gone through various paradigm shifts, but, alas, many of those paradigms have proved to be merely flashes in the pan. During this period, many slogans were advanced that supposedly represented fresh approaches to solving computer vision problems; slogans such as "active vision," "purposive vision," "reconstructive vision," "knowledge-based vision," "pure vision," etc., were bandied about. But apart from giving us a new vocabulary for explaining why some of the problems may not be solvable in the near future, these slogans have not amounted to much.

On the other hand, the vision systems that actually work today in the leading laboratories of the world are based on sound engineering principles. These systems use timeproven signal and image processing algorithms for extracting low-level information from images, estimation-theoretic approaches for dealing with uncertainty, graph-theoretic approaches for representing the extracted information by relational data structures, tools of computer graphics for object modeling and rendering, etc. What's interesting is that the success achieved in these systems is owing primarily to the workings of the underlying signal- and image-processing algorithms and to estimation-theoretic methods for coping with uncertainties. This is not to say that the high-level algorithmic control structures are dispensable, only that the systems tend to be more sensitive to the success of the signalprocessing end. I will also try to show that when computer vision does not work, it is primarily due to the fact that we do not know how to devise appropriate signal and image processing strategies for low-level feature extraction and that sometimes intractable problems become solvable by incorporating a human in the loop for helping out with low-level feature extraction.

I will show results from some state-of-the-art experiments in model-based vision for object recognition, for indoor mobile-robot navigation, for content-based image retrieval, etc. The robustness achieved by combining model-based reasoning with uncertainty management in these systems has resulted in applications that are expected to benefit industrial automation and medical diagnostics of the future. 


\section{Overview of Biopatheogen Detection Technology at LLNL}

Fred Milanovich 


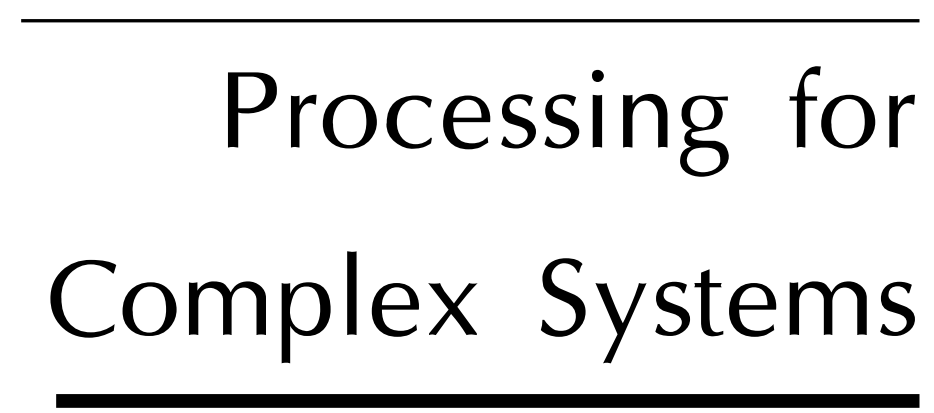




\title{
Overview of the Center for Complex Distributed Systems
}

\author{
David McCallen
}


P R O C E S S I N G F O R C O M P L E X S Y S T E M S

\section{Using signal processing techniques to improve finite element modeling}

\section{近 \\ CASIS Signal and Image Sciences Workshop}

November 11, 1999

Greg Burnett

Lawrence Livermore National Laboratory

Finite Element Models (FEM)

Ubiquitous in engineering, used to model everything from beer cans to experimental airplanes to skyscrapers

Have come increasingly into use as computing power has become cheaper and more widely available

They model continuous structures as a series of discrete elements that connect at nodes

Built using a standard set of elements and nodes plus a healthy portion of engineering judgement 
$\begin{array}{lllllllllllllllllllllll}P & \text { O C E S S I N G F O R C O M P L E X S Y S T E } & \text { M S }\end{array}$

Typical low order FEM

- Fifth order system of a five story building

- Each element has a stiffness and shear value

- Each node connects elements and has its own properties

- Linear model characterized by $\mathrm{M}, \mathrm{C}, \mathrm{K}$ matrices, describes structure with a set of ODEs

How are FEM traditionally tested and improved?

- Most of the testing involves the vibrational modes of the system under study

- For linear systems these are the eigenvectors and eigenfrequencies of $\mathbf{M}^{-1} \mathbf{K}$

- The calculated modes of the FEM are compared to the experimentally measured modes of the structure

- Based upon the differences in the mode shapes and frequencies, the model can be changed in an effort to match the modal response 

P R O C E S S I N G
F O R
C O M P L E X

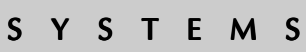

More systematic methods of FEM improvement:

- Gradient searches (Gauss-Newton)

-Good for signals with smooth minima

- Gan use with any cost function

- Optimal Linear Filtering

-i.e. Kalman Filters. Good with noisy signals, more slowly converging

-Essentially state estimators that can accept noisy measurements

- Optimal matrix, sensitivity based parameter update, and many others

What is our research objective?

- To improve Finite Element (FE) models systematically and effectively using state spacebased parameter identification methods 
P R O C E S S I N G F O R C O M P L E X S Y S T E M S

Why is this useful?

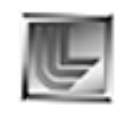

- The FEM update procedure may be used for two purposes:

1. To detect FEM mismatches (i.e. the model does not adequately describe the structure)

2. To detect damage to a structure if an adequate model for the structure exists

Why do we care?

- The possible applications of this technology are endless - FEM are extremely common in structural engineering

- Civil applications include the improved modeling of the response of bridges, buildings, and stadiums to events such as earthquakes or bombings

- Defense applications are many and varied 
$\begin{array}{lllllllllllllllllllllll}P & \text { O C E S S I N G F O R C O M P L E X S Y S T E } & \text { M S }\end{array}$

\section{What are the challenges of this project?}

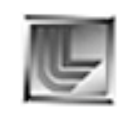

1. The models are usually large, consisting of a few hundred to a few thousand degrees of freedom

2. The number of measurements that we are able to make is usually much smaller than the DOF, on the order of $10-100$

3. The measurements are usually noisy

What are the challenges of this project?

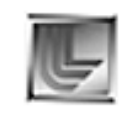

1. The models are usually large, consisting of a few hundred to a few thousand degrees of freedom

This can result in high computational expense

2. The number of measurements that we are able to make is usually much smaller than the DOF, on the order of $10-100$

3. The measurements are usually noisy 
$\begin{array}{lllllllllllllllllllllll}P & \text { O C E S S I N G F O R C O M P L E X S Y S T E } & \text { M S }\end{array}$

\section{What are the challenges of this project?}

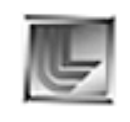

1. The models are usually large, consisting of a few hundred to a few thousand degrees of freedom

This can result in high computational expense

2. The number of measurements that we are able to make is usually much smaller than the DOF, on the order of $10-100$

This can cause observability and controllability problems - it is difficult to tell where the damage is precisely

3. The measurements are usually noisy

What are the challenges of this project?

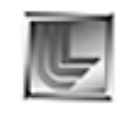

1. The models are usually large, consisting of a few hundred to a few thousand degrees of freedom

This can result in high computational expense

2. The number of measurements that we are able to make is usually much smaller than the DOF, on the order of $10-100$

This can cause observability and controllability problems - it is difficult to tell where the damage is precisely

3. The measurements are usually noisy

This can impede the ability of the algorithms to converge on the correct answer 
P R O C E S S I N G F O R C O M P L E X S Y S T E M S

How do we overcome the difficulties?

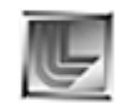

1. The models are usually large, consisting of a few hundred to a few thousand degrees of freedom

2. The number of measurements that we are currently able to make is usually much smaller, on the order of $10-100$

3. The measurements are usually noisy

How do we overcome the difficulties?

1. The models are usually large, consisting of a few hundred to a few thousand degrees of freedom

Reduce the model order through intelligent (human based) condensation

Use recursive algorithms that process data sequentially, reducing the amount of memory required

2. The number of measurements that we are currently able to make is usually much smaller, on the order of $10-100$

3. The measurements are usually noisy 

P R
C E S S I N G
F O R
C O M P L E X
$S$ S $S$ T $E$ E M S

\section{How do we overcome the difficulties?}

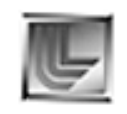

1. The models are usually large, consisting of a few hundred to a few thousand degrees of freedom

Reduce the model order through intelligent (human based) condensation

Use recursive algorithms that process data sequentially, reducing the amount of memory required

2. The number of measurements that we are currently able to make is usually much smaller, on the order of 10-100

Estimate the unmeasured data with the model and place sensors at positions where there is the most uncertainty

Interpolate the measurements intelligently

3. The measurements are usually noisy

\section{How do we overcome the difficulties?}

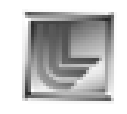

1. The models are usually large, consisting of a few hundred to a few thousand degrees of freedom

Reduce the model order through intelligent (human based) condensation

Use recursive algorithms that process data sequentially, reducing the amount of memory required

2. The number of measurements that we are currently able to make is usually much smaller, on the order of 10-100

Estimate the unmeasured data with the model and place sensors at positions where there is the most uncertainty

Interpolate the measurements intelligently

3. The measurements are usually noisy

Use a statistically based estimation algorithm 


\section{Damage Detection}

- Four levels of damage detection

- Level 1: Is the system damaged?

- Level 2: Where is the damage located?

- Level 3: What type of damage is present?

- Level 4: What is the extent of the damage?

- Historically only levels 1-2 addressed, large amounts of damage needed to localize

- Usually done by comparing mode shapes and frequencies, but some signal processing techniques such as LPC modeling used

\section{System Identification}

- System identification involves level 3-4 damage detection - determining the amount and type of damage

- Output from accelerometers on actual structures is used to update FEM with values of $K$ that are more appropriate tot he structure

- Very little can be done presently to update models systematically - especially with low observability. It is a mix of art and science. 

P R
C E S S I N G
F O R
C O M P L E X
$S$ Y S T E M S

\section{Steps of progress for system ID}
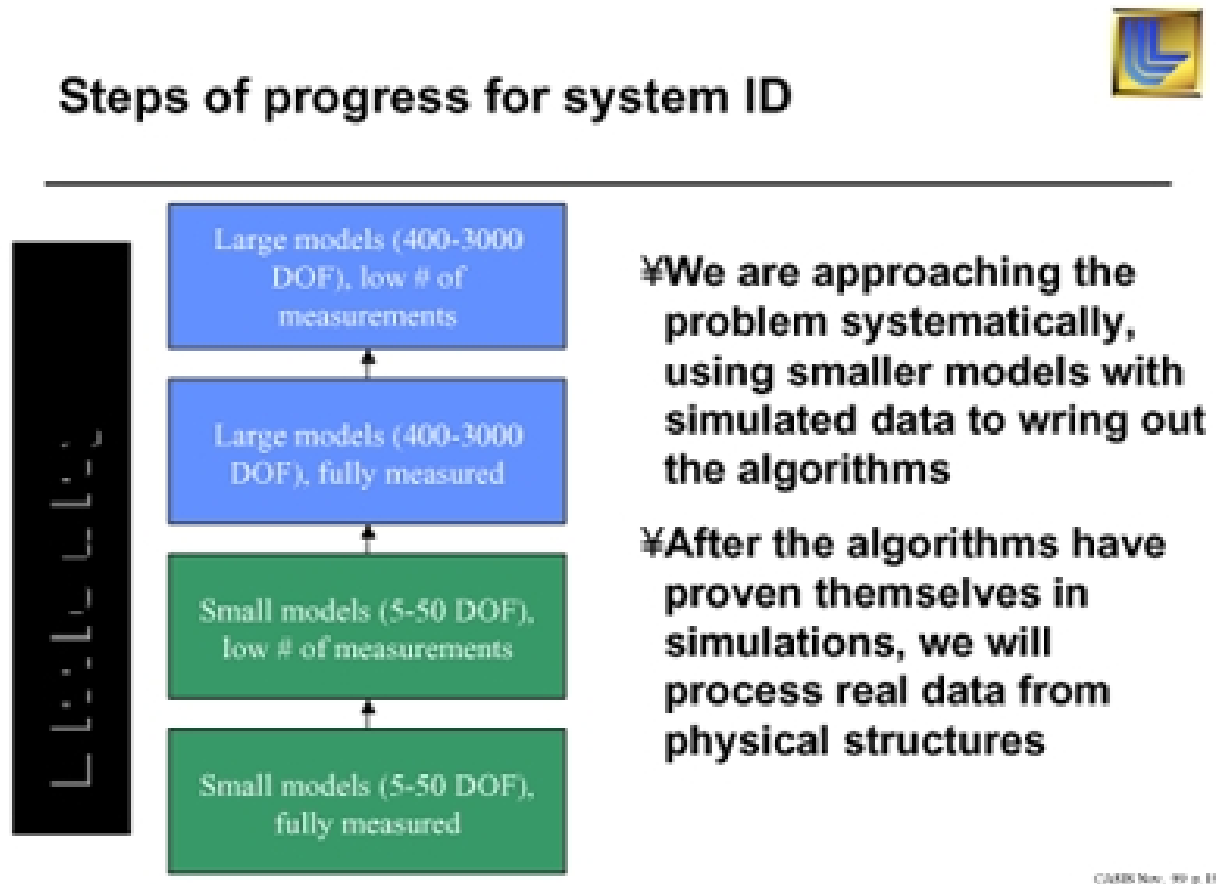

$¥$ We are approaching the problem systematically, using smaller models with simulated data to wring out the algorithms

$¥$ After the algorithms have proven themselves in simulations, we will process real data from physical structures

\section{We have made extensive use of a damped} Gauss-Newton search algorithm in our initial studies

It uses a Gaussian approximation to the Newton method to search for a minimum of the least-squares search criterion

Its advantages are:

-Quickly converges on relatively linear minima with small residuals

- Guaranteed to converge on a minima

-Widely used and available (Matlab s PEM is similar)

Its disadvantages are:

\footnotetext{
-May not converge to a global minima

-May not converge if residuals large, system too nonlinear

-Noise can prevent convergence
} 
P R O C E S S I NG F O R C O M P L E X S Y S T E M S

So what have we learned?

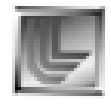

Parameter identification is possible for both fully measured and partially measured systems, but it is critical to select the correct measurement nodes

For large DOF systems, Gauss-Newton search may not be the best algorithm

High DOF systems span large dimensional space, many locals minimums possible

Example illustrating the effect of sensor placement on identification accuracy

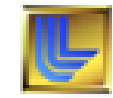

(10 DOF, 5 rotational, 5 translational, sim data, no noise)

We were able to detect large (up to $2 / 3$ of the original value) perturbations

With 5 measurements accurate to better than $0.01 \%$

With 4 measurements accurate to better than $1 \%$

With 3 measurements accurate from less than $1 \%$ (9 cases) to $32 \%$ ( 1 case), depending on states measured

With 2 or 1 measurements, the mean error could increase to $80 \%$ or more

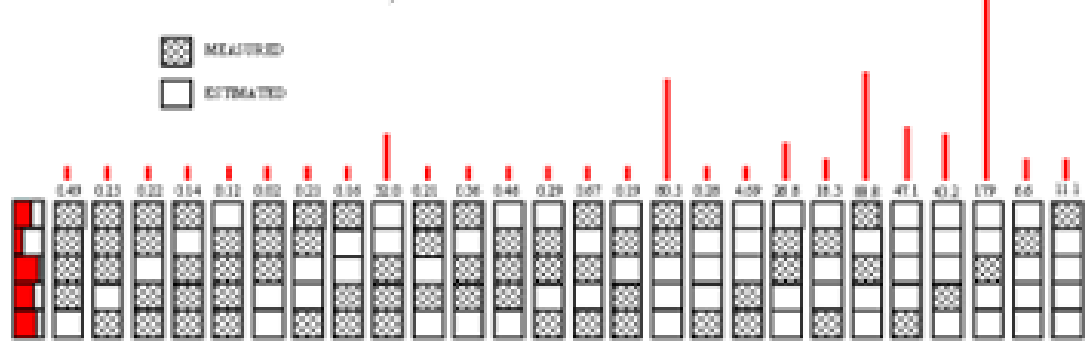



P R
C E S S S I N $\mathrm{N}$
F O R
C O M P L E X
$S$ S $S$ T $E$ E M S

\section{Typical low order FEM}
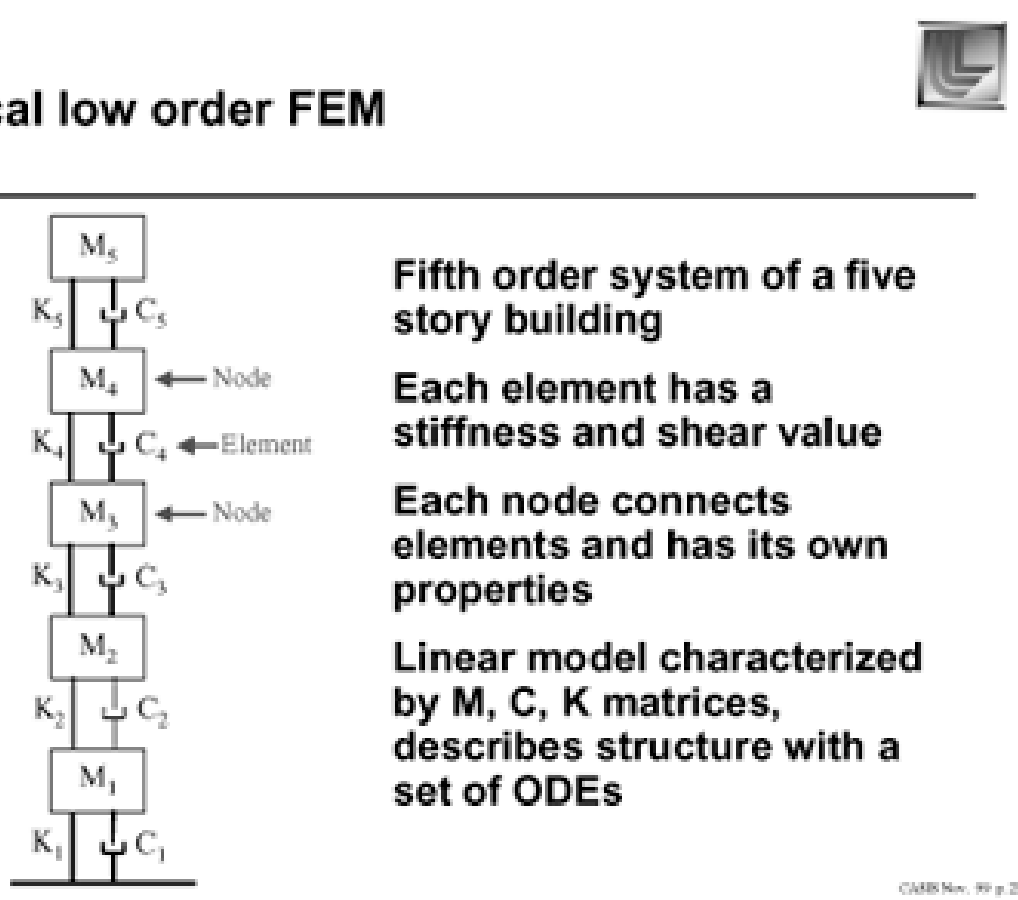

Fifth order system of a five story building

Each element has a stiffness and shear value

Each node connects elements and has its own properties

Linear model characterized by $\mathrm{M}, \mathrm{C}, \mathrm{K}$ matrices, describes structure with a set of ODEs

\section{DOF results (only 5 translational states measured)}

Good news: Can find and partially correct element errors near measurement points

Bad News: Elements far from measurement points basically unaffected

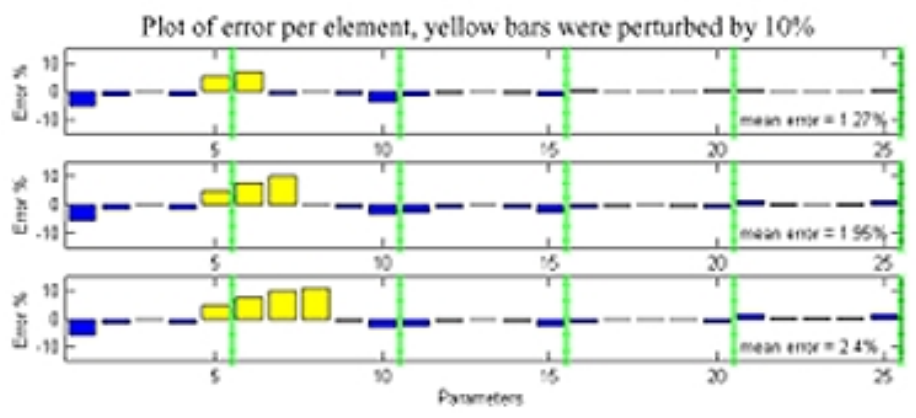


P R O C E S S I N G F O R C O M P L E X S Y S T E M S

For systems that are not measured at every node, damage detection and updates for nonmeasured nodes are difficult.

Instead of a direct connection, there exists a zone of influence around the measured node

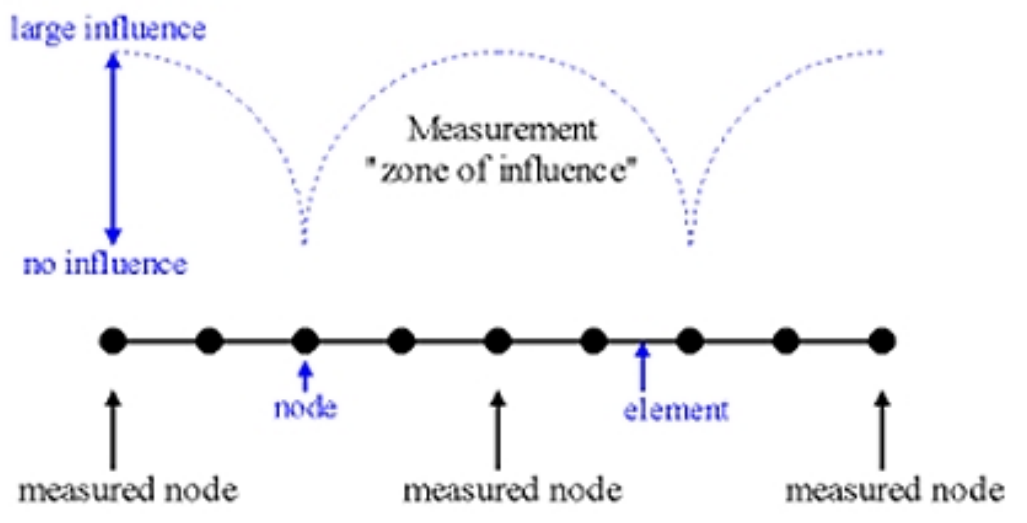

Conclusions on simulated system ID 

$P$ R O C C E S S I N G
F O R
C O M P L E X
$S \quad Y \quad S \quad T \quad E \quad M \quad S$

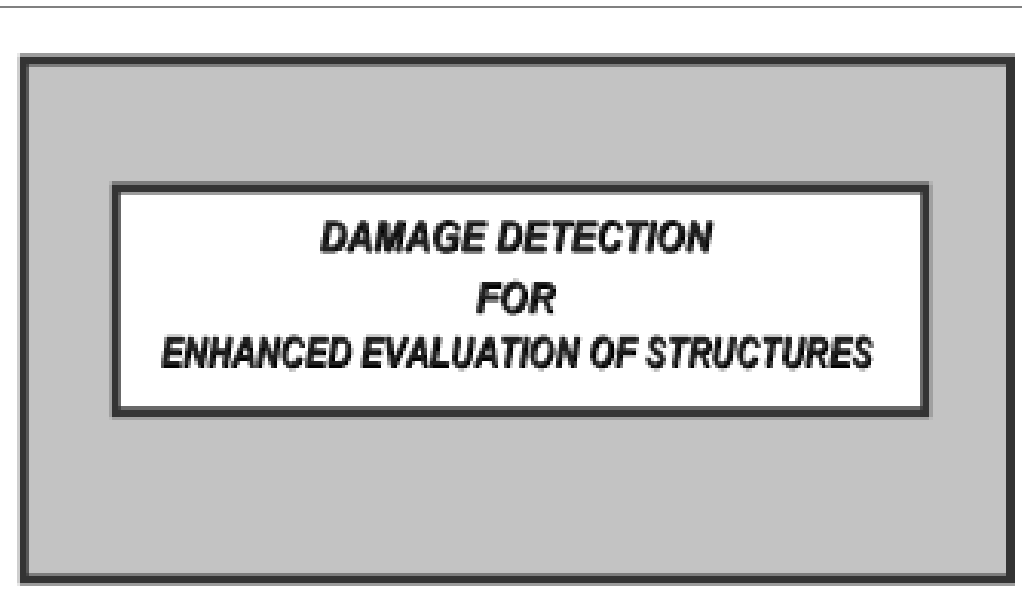

G. Clark, G. Burnett, DCCallen

\section{CASIS Workshop}

Knoteratilitim

LAWRENCE LIVERMORE NATIONAL LABORATORY

\section{ORGANIZATION}

- INTRODUCTION

- MODELS

- dAMAGE DETECTION

- PROCESSING RESULTS

- SUMMARY

LAWRENCE LIVERMORE NATIONAL LABORATORY 
P R O C E S S I NG F O R C O M P L E X S Y S T E M S

\section{Damage detection is achieved using a model-based processor}

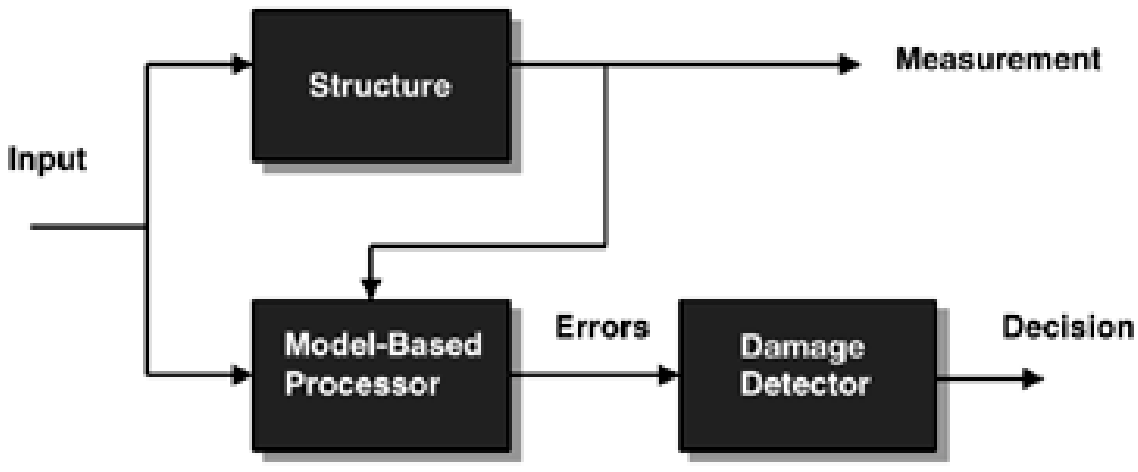

LAWRENCE LIVERMORE NATIONAL LABORATORY

MBP design for vibrating structures requires that:

we seek techniques to incorporate:

* structural models

* sensor (measurement) models

* noise models (ambient and measurement)

into the processor

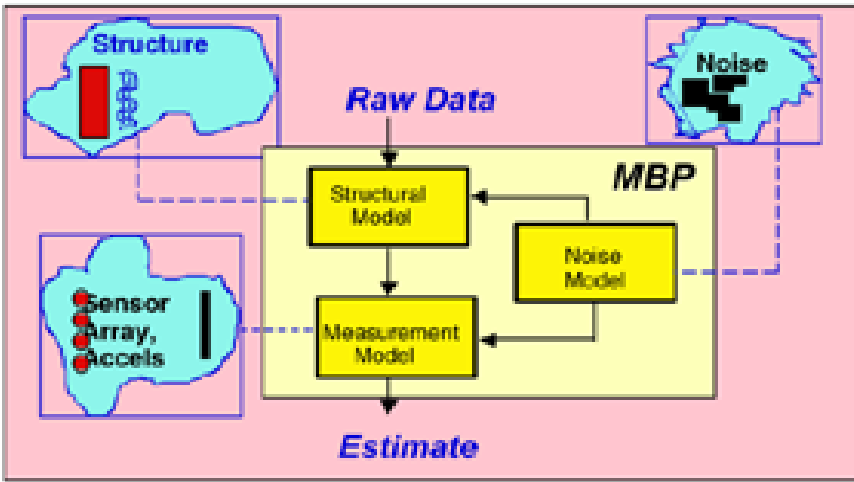

LAWRENCE LIVERMORE NATIONAL LABORATORY 

P R
C E S S I N G
F O R
C O M P L E X
$S \quad Y \quad S \quad T \quad E \quad M \quad S$

The choice of MODEL determines the processing algorithm:

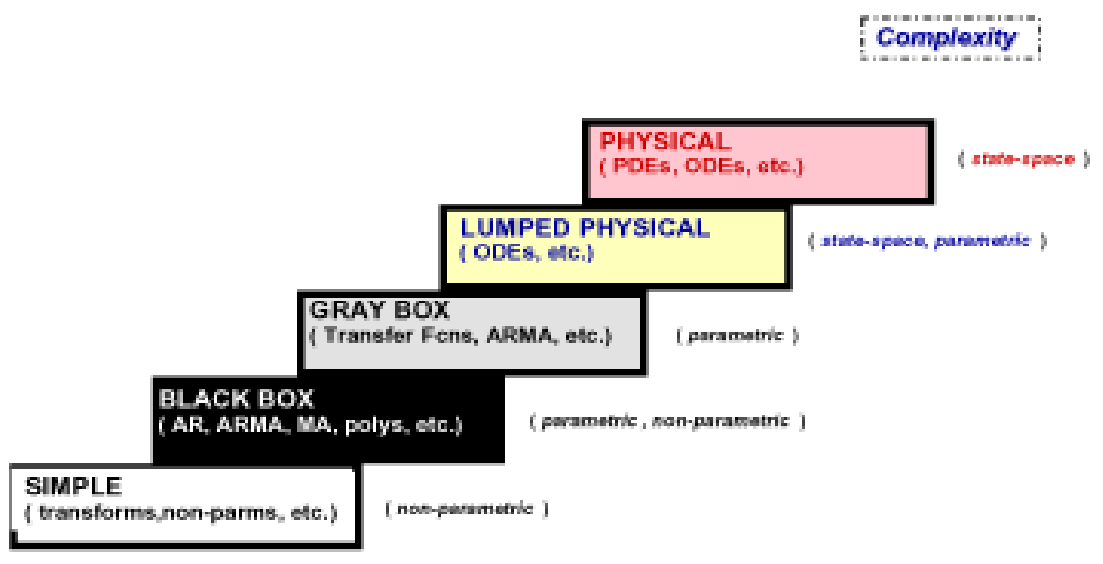

Simplicity

LAWRENCE LIVERMORE NATIONAL LABORATORY

The detection, classification and prediction of structural failures is possible with the model-based approach

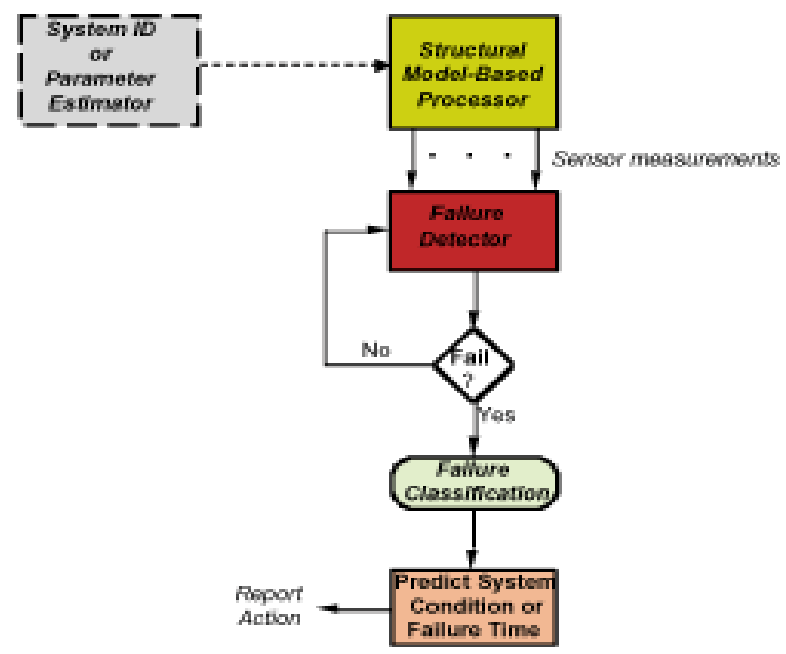

LAWRENCE LIVERMORE NATIONAL LABORATORY 

P R O
C E S S I N G
F O R
C O M P L E X
$S \quad Y \quad S \quad T \quad E$ M S

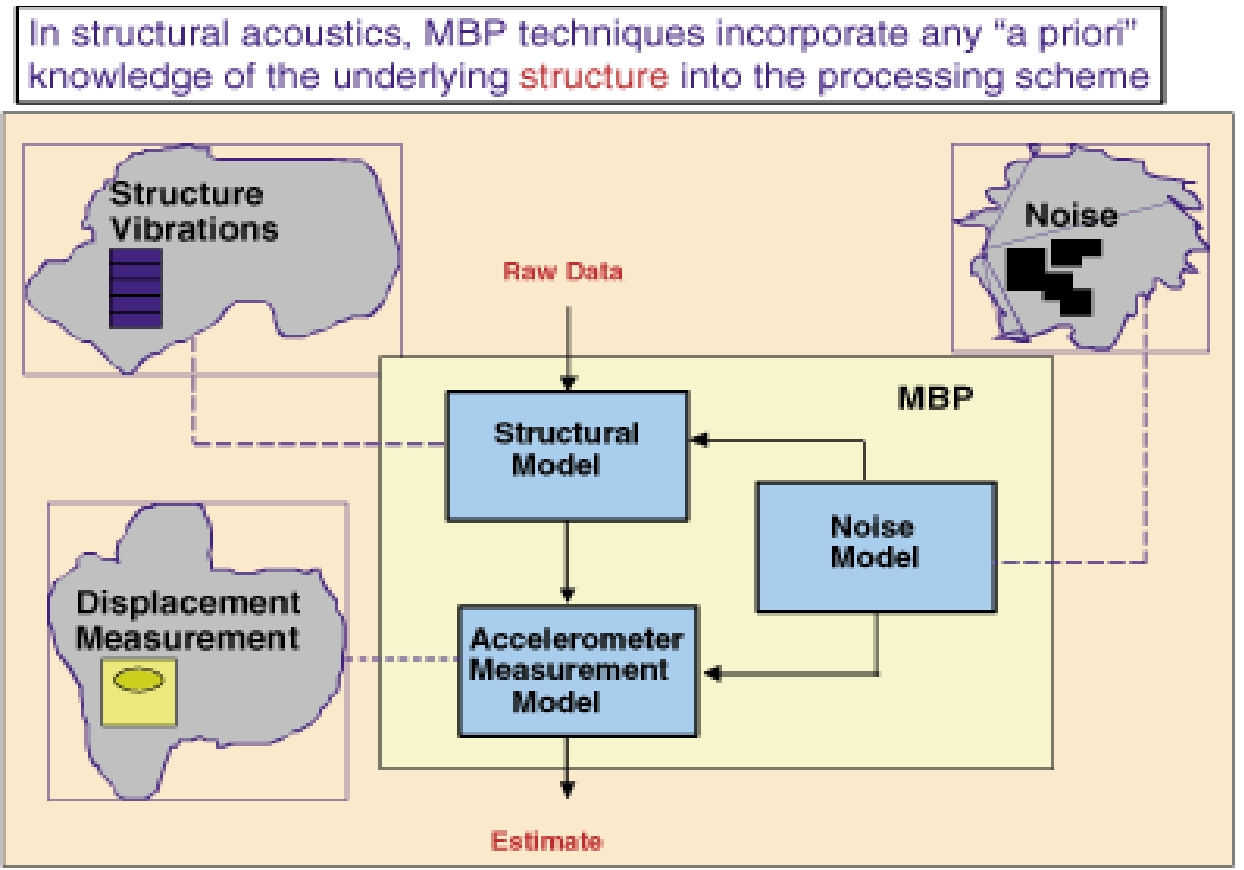

LAWRECE LIVERMOAE NATIONAL LABOAATORY

When the dynamic behavior of the structure is normal, the MBP "innovations" are "white"

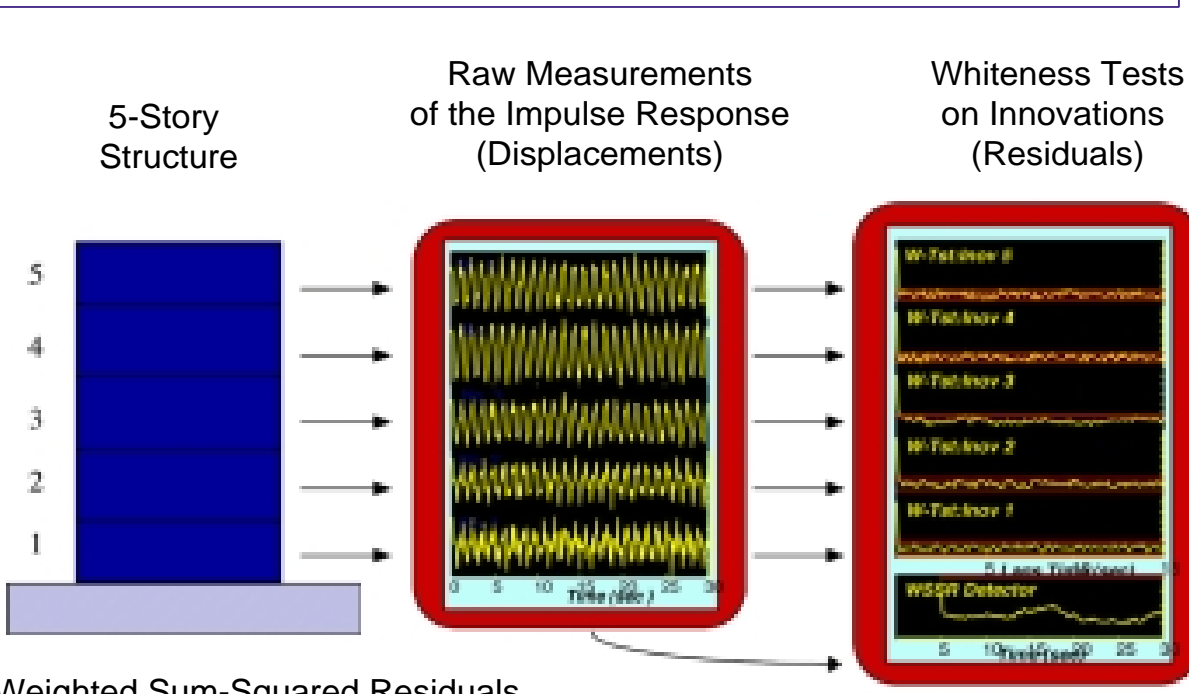

Weighted Sum-Squared Residuals

$($ WSSR $)=$ Aggregation of all the whiteness tests

\section{LAWRENCE LIVERMORE NATIONAL LABORATORY}



P R O C
C E S S S I N
F O R
C O M P L E X
$S \quad Y \quad S \quad T \quad E \quad M \quad S$

When the dynamic behavior of the structure is normal, the MBP "innovations" are "white"

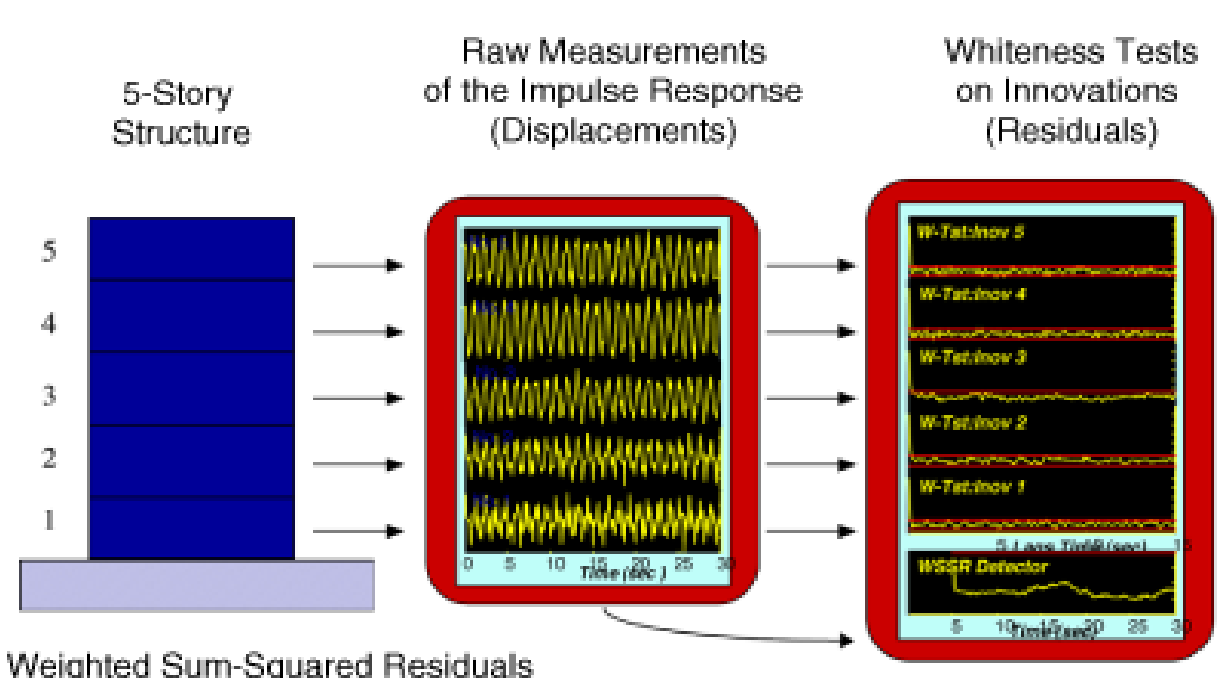

Weighted Sum-Squared Residuals

(WSSR) $=$ Aggregation of all the whiteness tests

LAWRENCE LIVERMORE NATIONAL LABORATORY

Comparing PSDs of the innovations (residuals) also shows the damage resonances

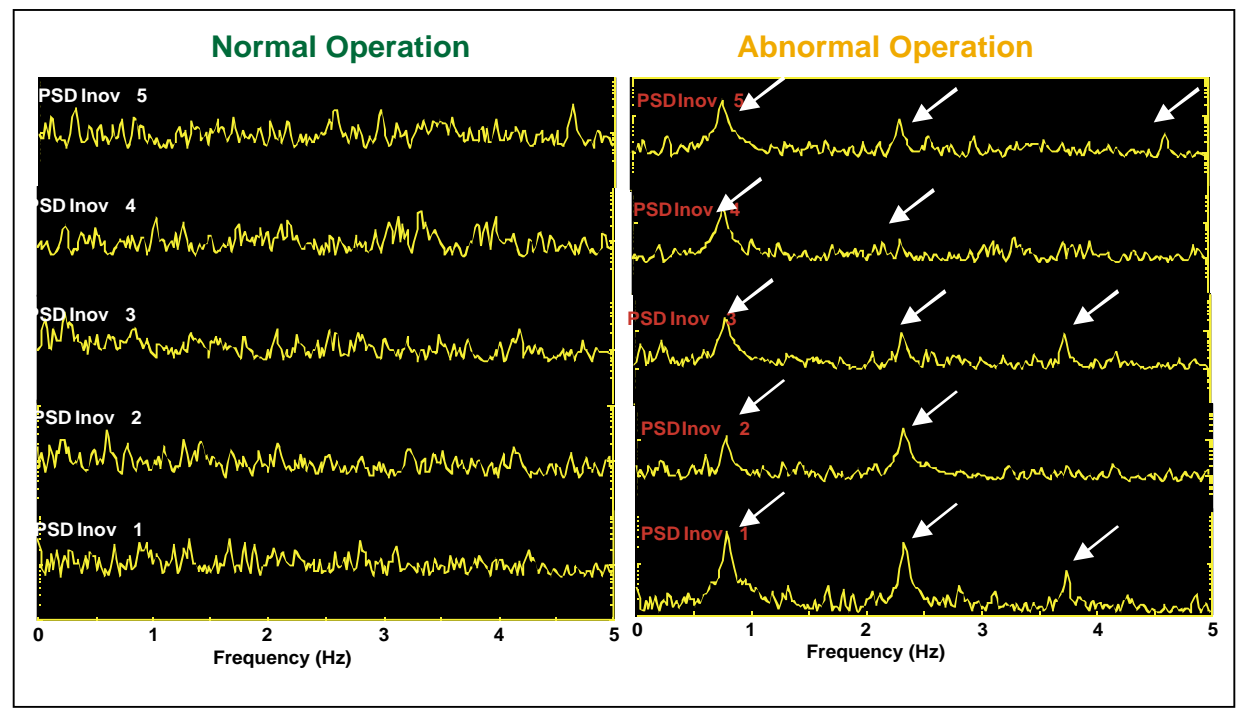

LAWRENCE LIVERMORE NATIONAL LABORATORY 

P $\quad$ R O
C E S S I N G
F O R
C O M P L E X
$S \quad Y \quad S \quad T \quad E \quad M \quad S$

\section{Parabolic Reflector for Radar Mine Detection}

S. Azevedo, T. Rosenbury, G. Dallum, M-L Liu, M. Vigars, Lawrence Livermore National Laboratory(LLNL)

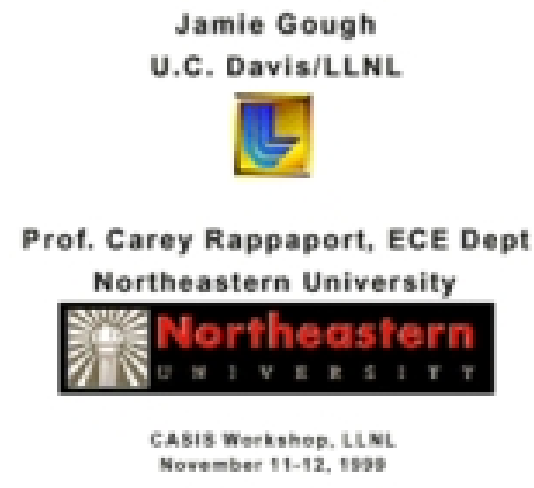

LLNL has been researching radar mine detection techniques using new technologies

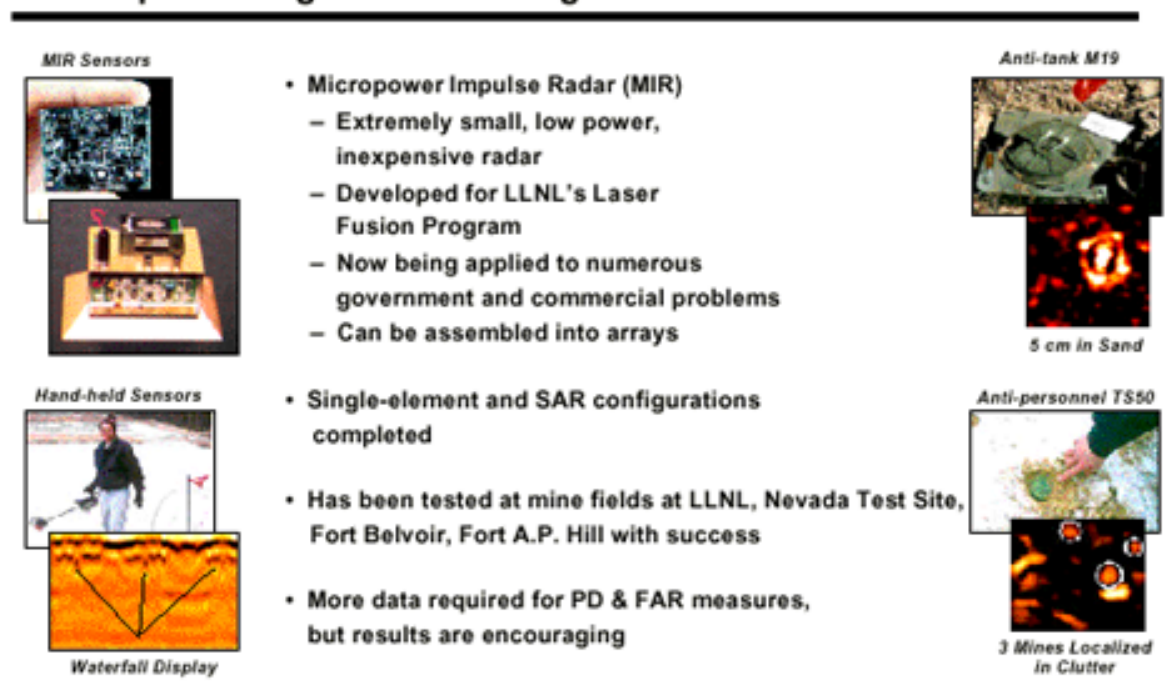

\section{This is the smallest known radar, hence it can be assembled into imaging arrays}



$P$
F O R
C O M P L E X
$\begin{array}{llllllllllllllllllll}S & Y & S & T & E & M & S\end{array}$

\section{Outline}

- Mine detection problem .- forward-looking, lightweight AP mine detector

- Ground-clutter minimization -. plane wave vs. point source impulse excitation

- Offset paraboloid reflector

- FDTD modeling

- Multistatic receiver array for time-domain focusing

- Antenna fabrication

- Experimental results

- Next steps

A strong specular ground reflection hampers the effectiveness of radar mine detection

- Specular reflection from the ground depends only on incidence angle, frequency content, and wave polarization, not position or source.

- For flat ground with non-normal incidence, reflection scatters in forward direction.

- Plane wave excitation generates scattered signals that are easier to distinguish from rough surface clutter.

- Parabolas convert circular waves to plane waves.

- In the nearfield, a paraboloidal reflector generates a (non-uniform) plane wave across its aperture.

- An offset paraboloidal section must be used to avoid blockage and scattering by transmitting feed.

- Trade-offs between reflector size and grazing incidence angle -- and height above ground versus forward look -- must be made. 
P R O C E S S I NG F OR C O M P L E X S Y S TE M S

A novel Forward-Looking "Handheld" AP Mine Detector with Plane Incidence Wave Excitation

Offset Paraboloid Ray Paths

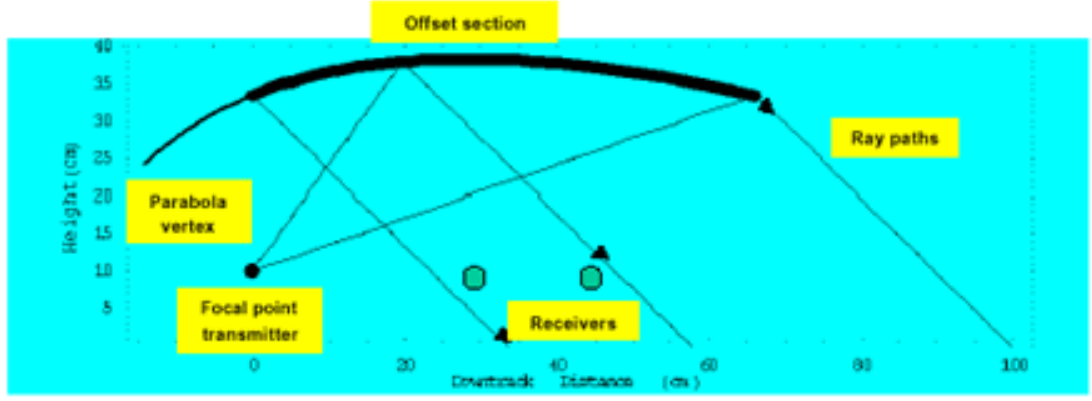

3D views of offset paraboloid

Top
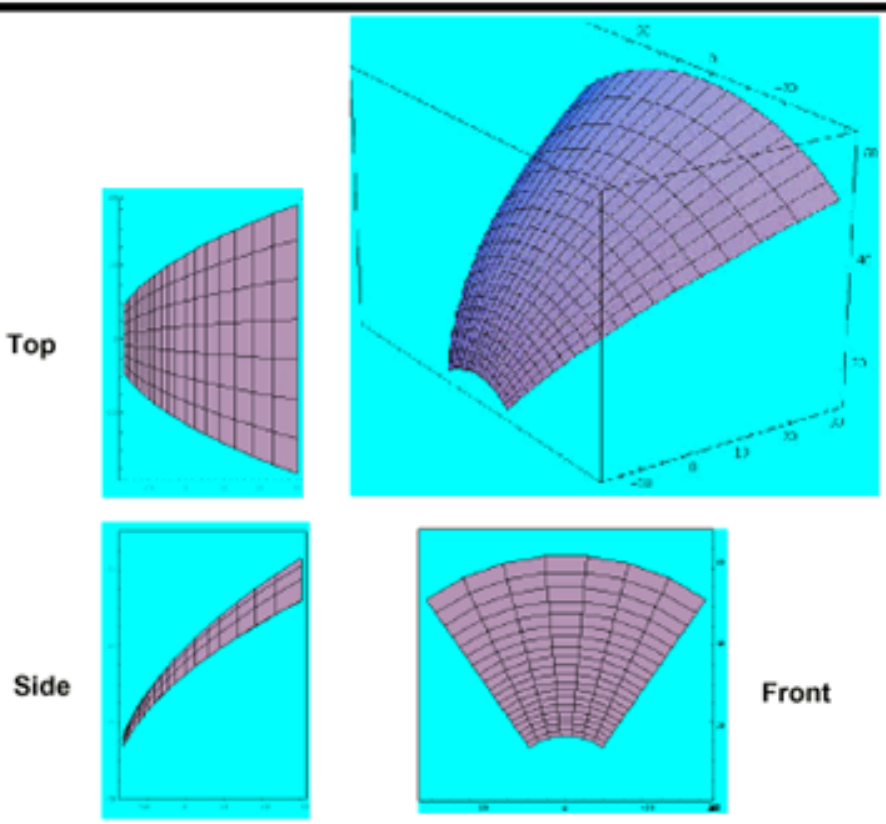

Front

$\underset{\text { antman }}{\text { antan }}$ 

P R
C E S S I N G
F O R
C O M P L E X
$S \quad Y \quad S \quad T \quad E \quad M \quad S$

\section{2-D FDTD Simulation of Parabolic Reflector Generated}

V-Pol. Planar Incident Wave

\section{Non-Metallic Mine $10 \mathrm{~cm}$ Below Flat Surface}
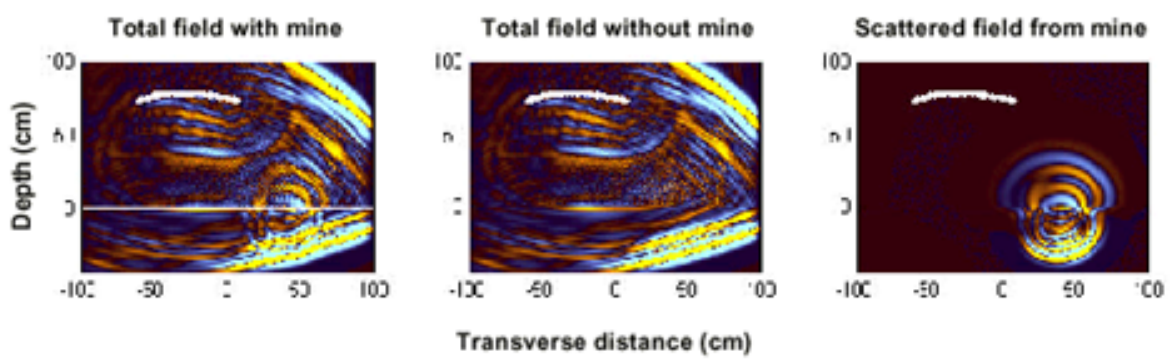

19.

\section{2-D FDTD Simulation of Parabolic Reflector Generated V-Pol. Planar Incident Wave}

Non-Metallic Mine $10 \mathrm{~cm}$ Below Rough Surface
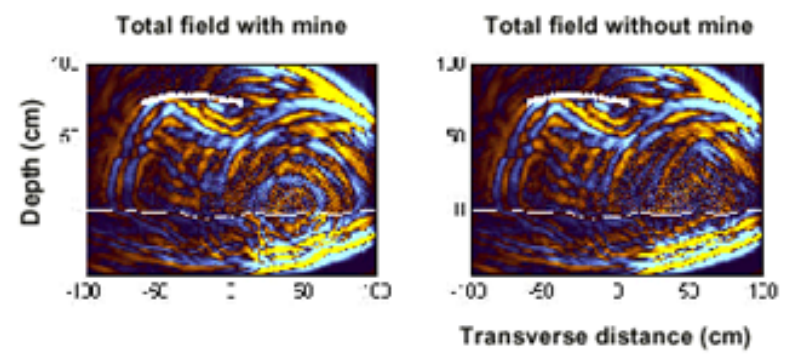

Scattered field from mine

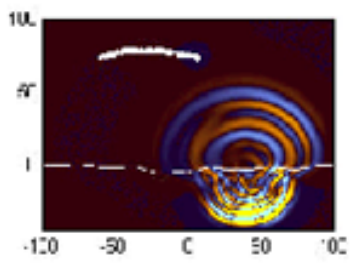

G 

P R O
C E S S I N G
F O R
C O M P L E X
$S$ Y $S$ T $E$ M $S$

The parabolic reflector system was built and tested as a prototype detection device
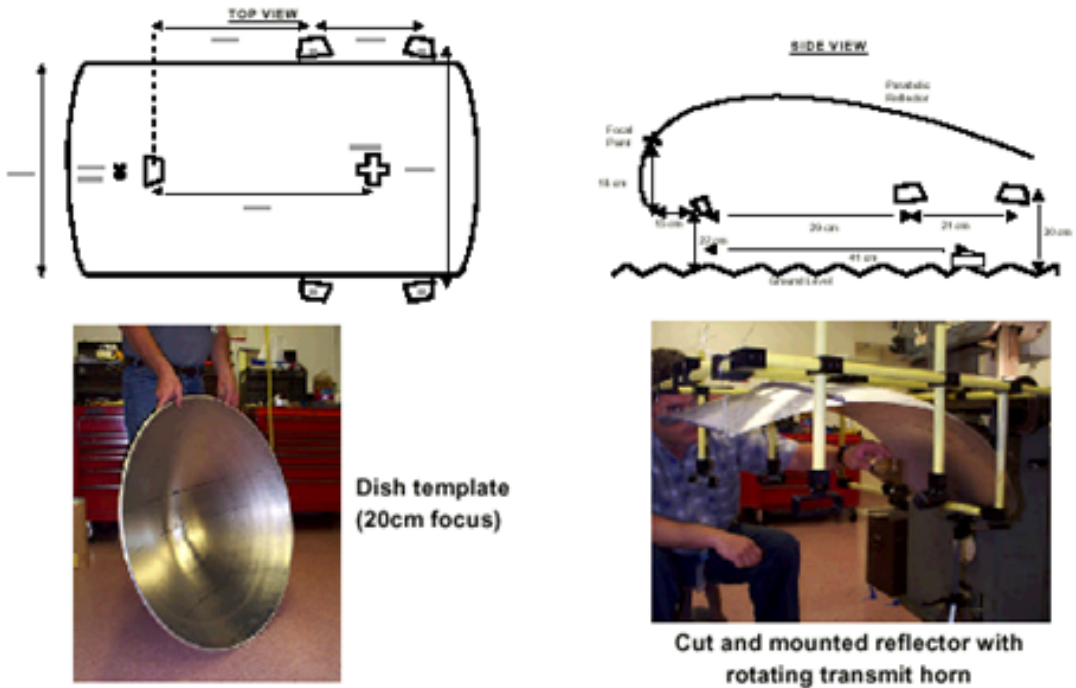

Cut and mounted reflector with rotating transmit horn

Four micropower radar receivers, in array configuration, were mounted on the reflector
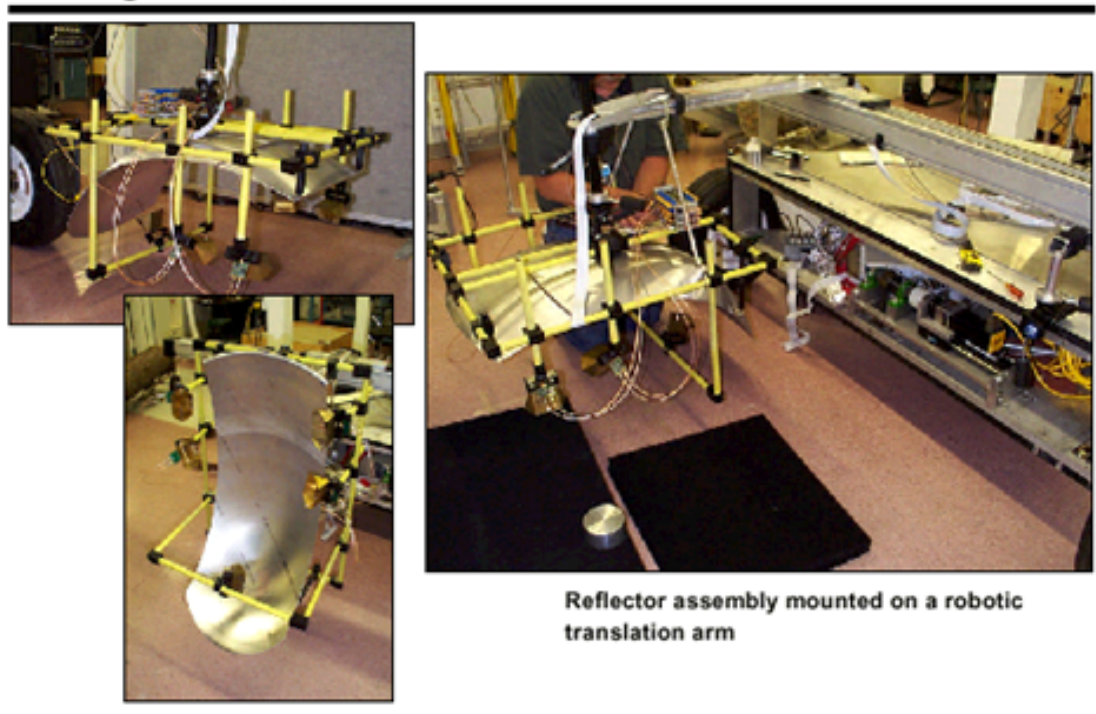

Reflector assembly mounted on a robotic translation arm 

$\begin{array}{llllllllll}P & R & O & C & E & S & S & I & N & G\end{array}$
F O R
C O M P L E X
$S$ Y S T E M S

\section{The MIR pulse is stable and wideband}

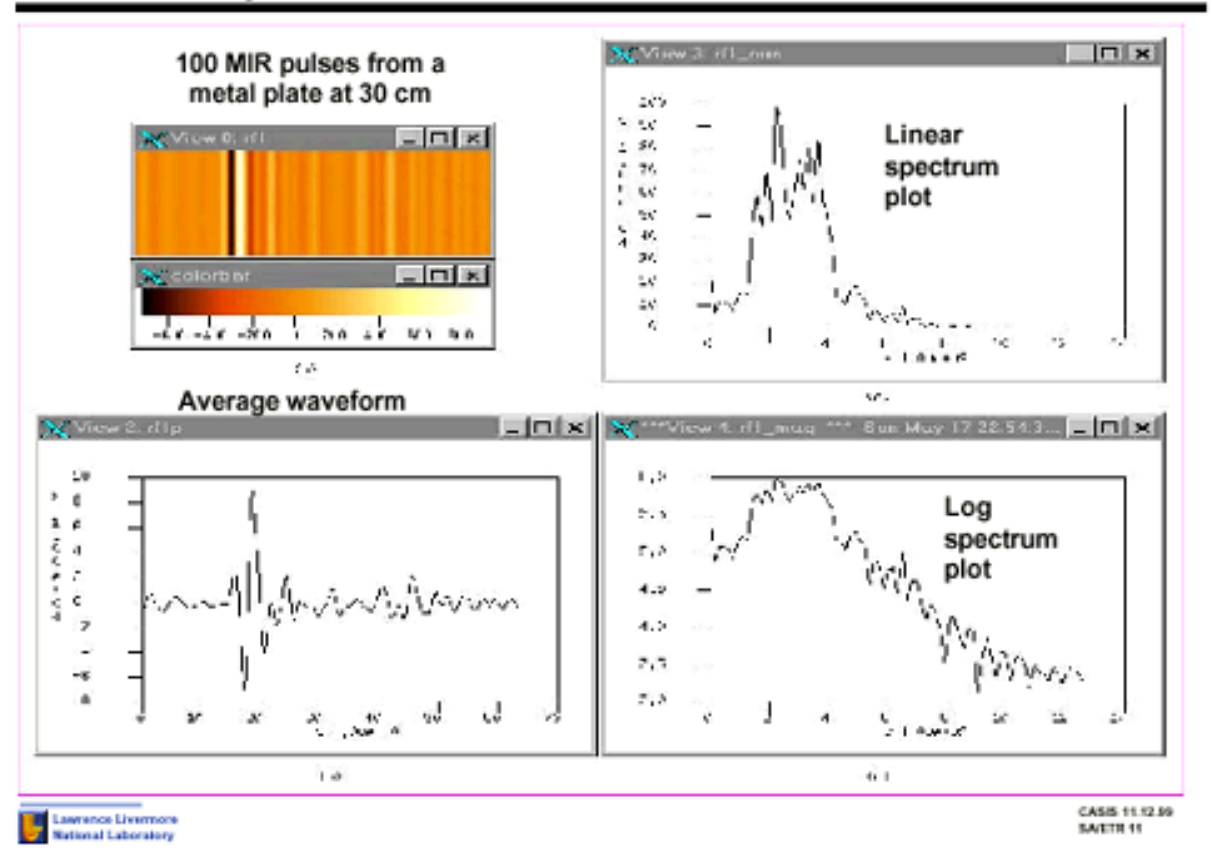

\section{Calibration showed near-constant illumination in the field of view}

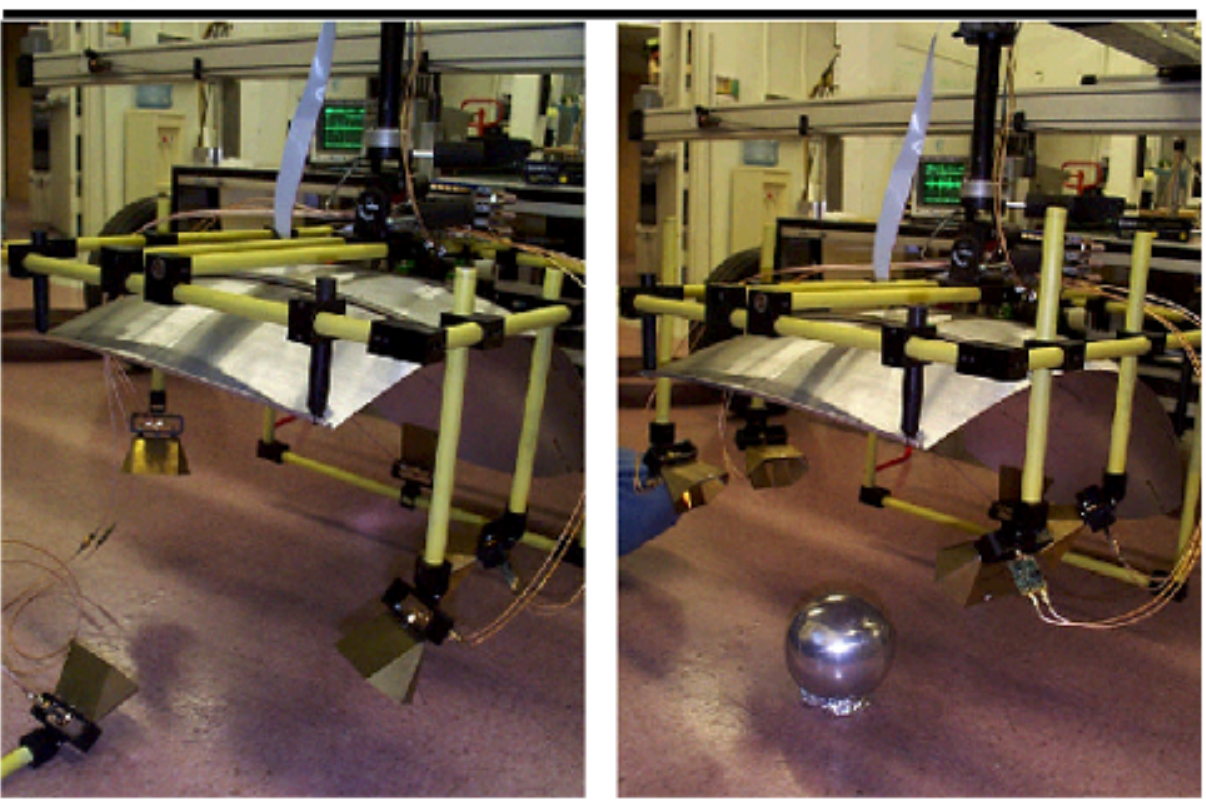

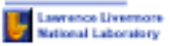

Receivers were placed to maximize the return 

P $\quad$ R O O C
C E S S I N G
F O R
C O M P L E X
$S$ Y $S$ T $T$ E M S

\section{Sphere and mock-mine objects were tested in three soil types - sand, gravel, top-soil (dirt)}
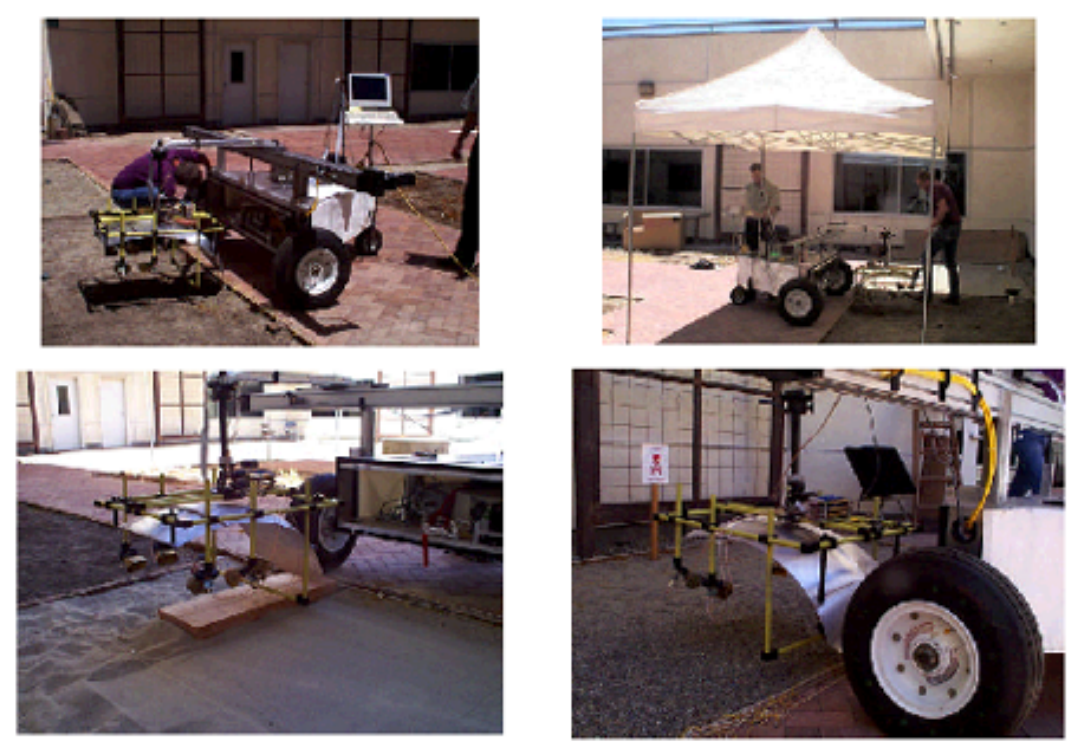

可

The first-surface echo is greatly reduced with the reflector vs a monostatic radar

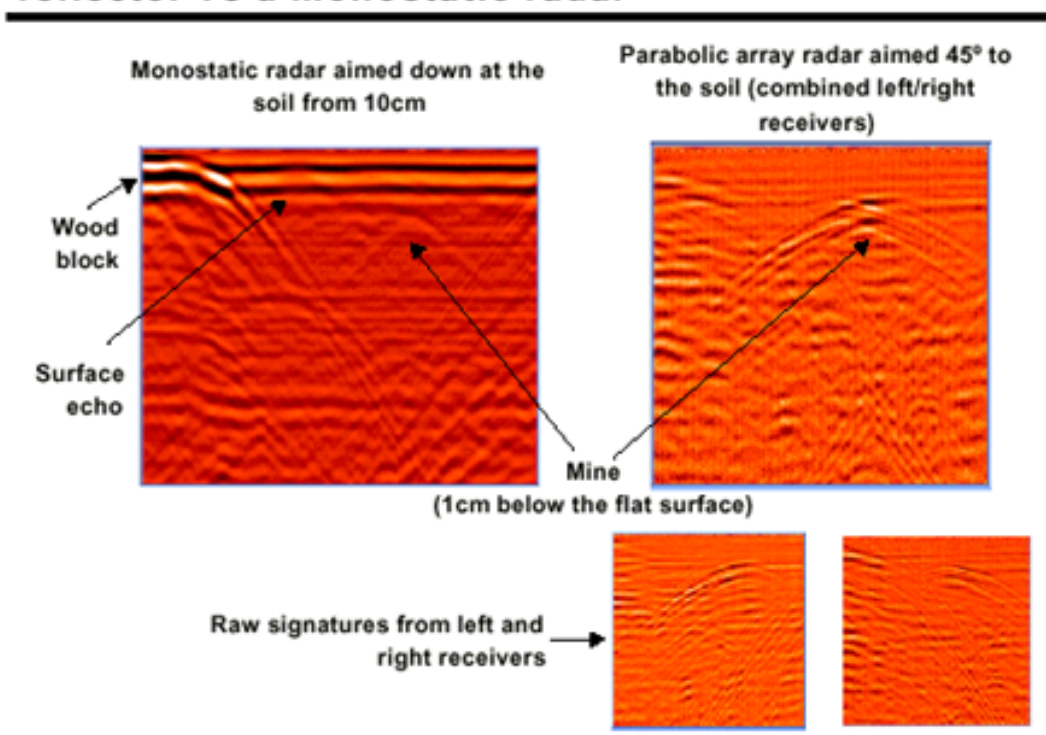



P
RO C
C E S S S I N G
F O R
C O M P L E X
$S \quad Y \quad S \quad T \quad E \quad M \quad S$

\section{A metal sphere was used for calibration}
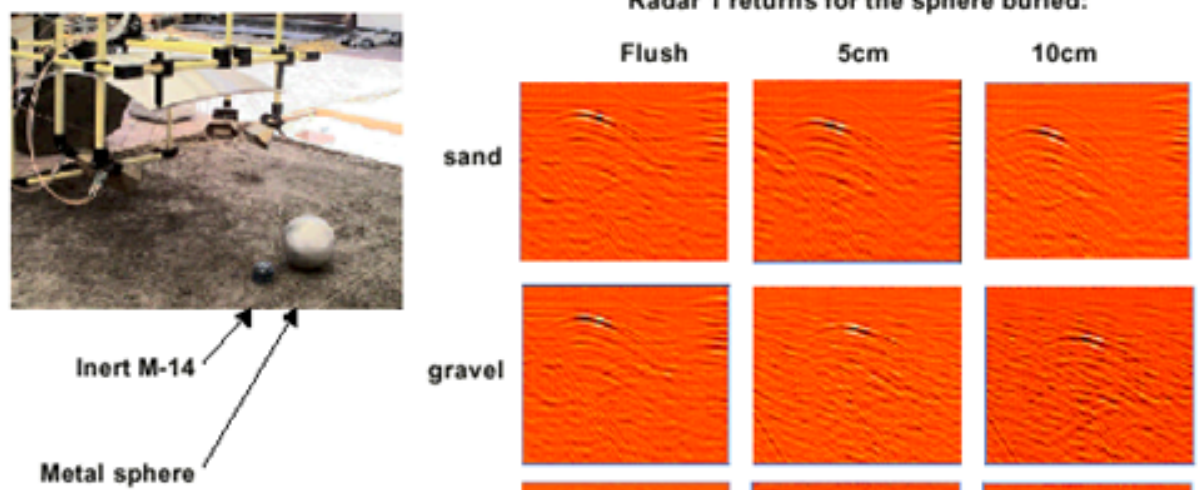

gravel
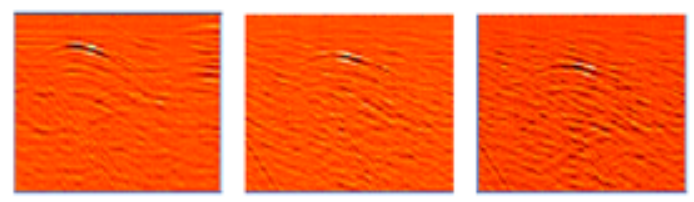

dirt
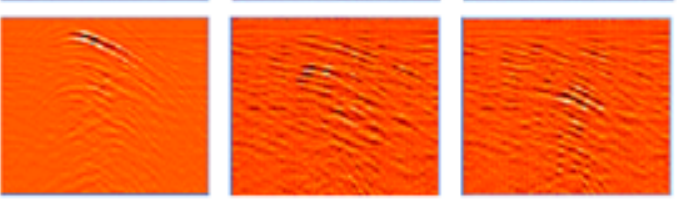

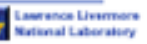

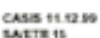

Results in 2D for the inert mine will need

improvement

Radar 1 returns for the mine buried:

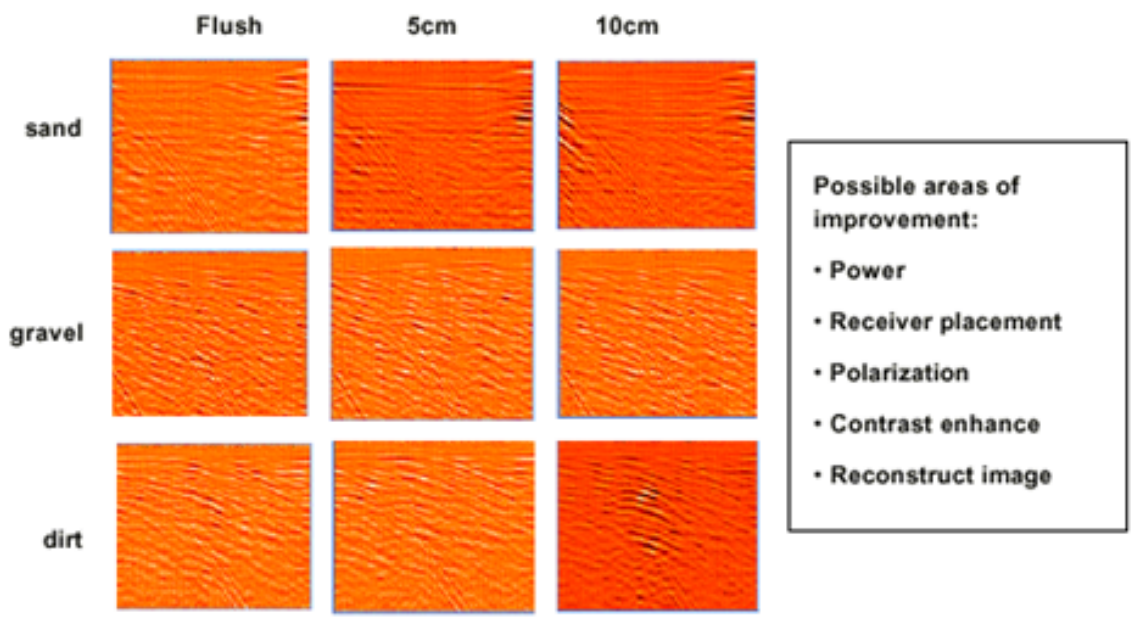



P R O C E E S S I N G
F O R
C O M P L E X
S $\quad Y \quad S \quad T \quad E \quad M \quad S$

\section{Rough-surface tests in the sand showed the most possibility for $\mathrm{S} / \mathrm{N}$ improvement}
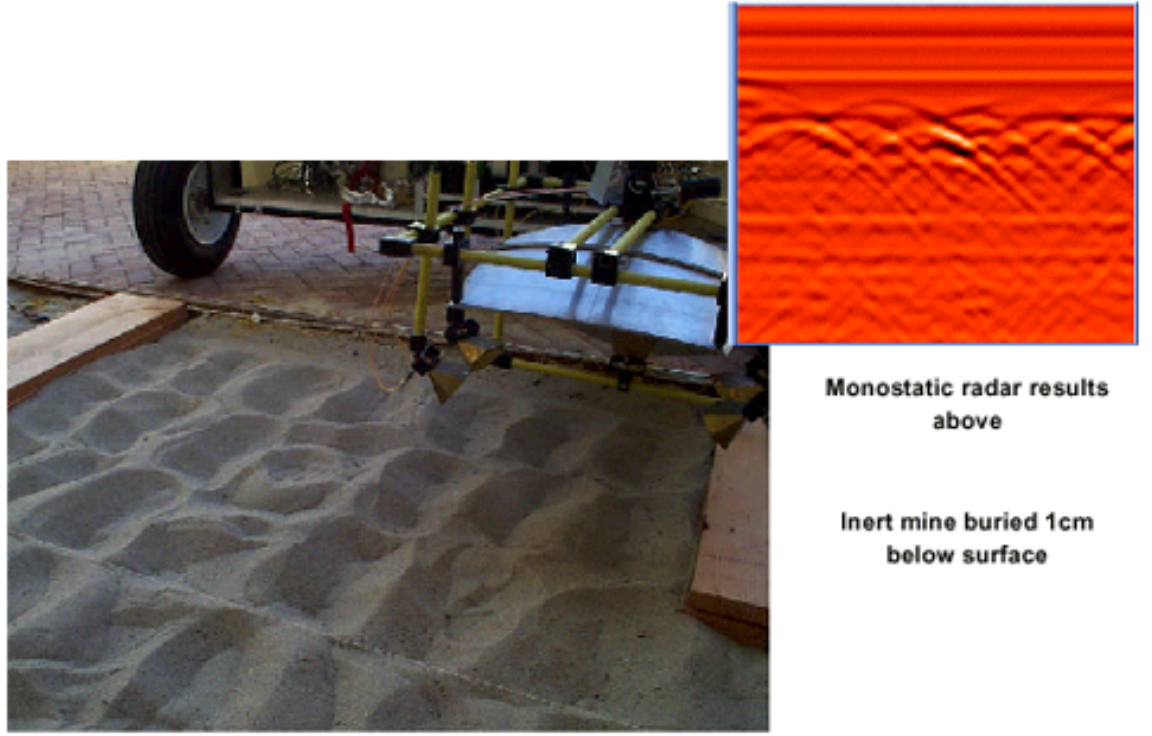

Inert mine buried $1 \mathrm{~cm}$ below surface

Rough-surface tests in the sand showed the most possibility for $S / N$ improvement (cont)

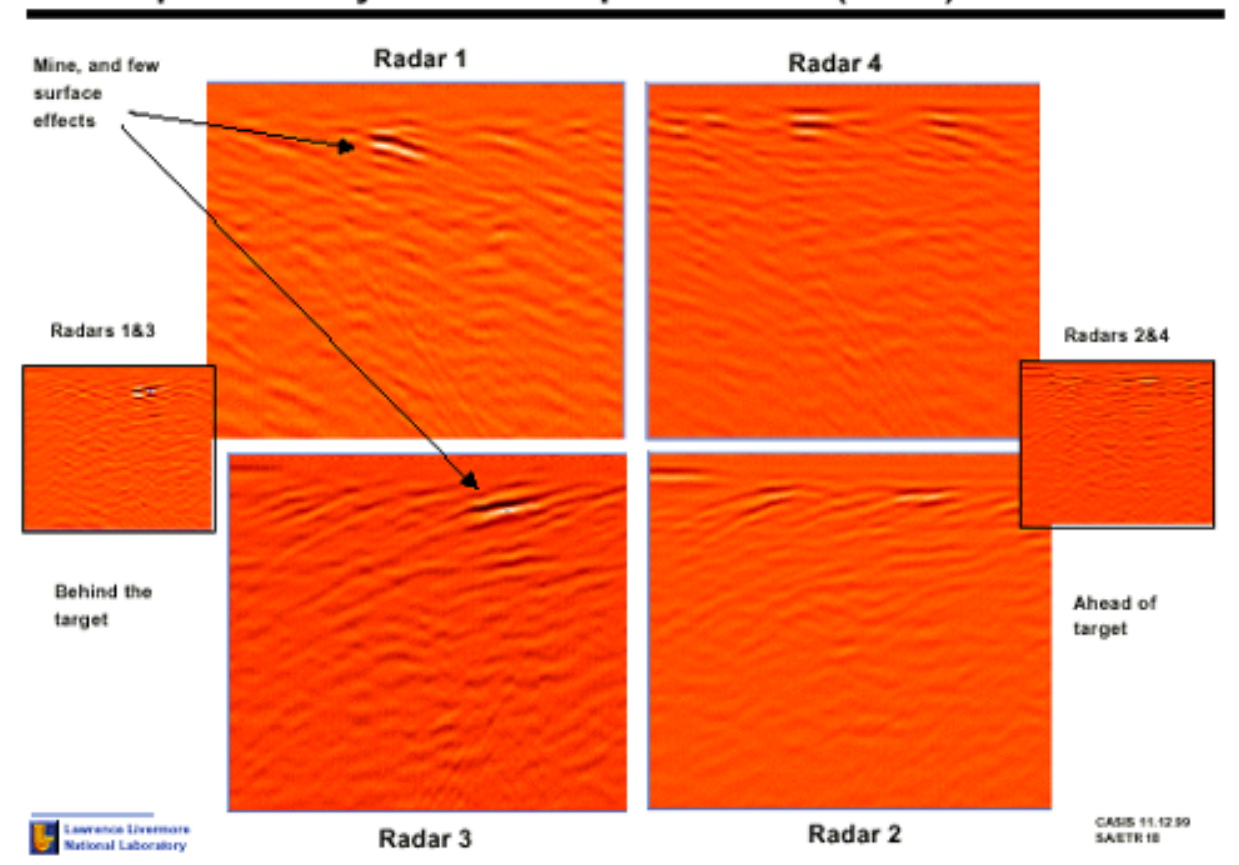



P R O C E S S I N G
F O R
C O M P L E X

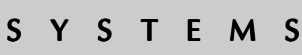

\section{Preliminary results show promise for the NU-designed parabolic reflector array antenna}

- Reflector provides good gain over large area

- Wavefront is measured to be planar in that area

- First surface echo is reduced greatly (over monostatic results)

- Placement and orientation of receiver antennas has an effect, but more research is needed

- Combining the delayed receiver waveforms improves the signal-toclutter ratio

- Metal sphere (for calibration) can be detected in all soils at all depths

- Rough surface results show improved detection with reflector

- Image reconstruction algorithms need to be developed for the reflector geometry and to improve the detection 


\title{
Broad Band Acoustic Ranging and Velocimetry
}

\section{David Chambers, Karl Fisher, Brian Guidry, David Erskine}

Acoustic ranging has a long history of development beginning with sonar in World War II and continuing with the advent of medical ultrasound. Acoustic velocimetry (velocity measurement) is not as common but has been used to measure blood flow in the body. Simultaneous measurement of range and velocity is used in some medical ultrasound imaging but the resolution is coarser than with conventional medical imaging (ranging only). In the conventional approach to acoustic ranging and velocimetry, short broad bandwidth pulses provide better range resolution while long narrow bandwidth pulses provide better velocity estimates.

In this work we apply a new optical technique developed by Dave Erskine for white light ranging and velocimetry to the acoustic case. We generalize the technique to coherent broad band pulses and test it with spheres pulled through a specially designed water tank. We compare the sensitivity of the broad band technique with the conventional narrow band approach for velocity measurements. These experiments show that both range and velocity can be accurately measured with broad band pulses without degrading the range resolution. The technique can be easily applied to conventional sonar or radar, and medical imaging.

\section{Broad Band Acoustic Ranging and \\ Velocimetry}

\author{
David Chambers, Karl Fisher, Brian Guidry, \\ David Erskine \\ CASIS Workshop \\ November 12, 1999
}


S I G N A L P R O C E S S I N G

Conventional ranging and velocimetry trade accuracy of range estimates with accuracy of velocity estimates

Accurate range estimates favor broad band (short) pulses

Accurate velocity (Doppler shift) estimates favor narrow band (long) pulses

Current systems (e.g. Doppler medical ultrasound) trade resolution (range estimation) for Doppler sensitivity

Sonar systems typically estimate velocity by tracking range over time.

Erskine $s$ white light velocimetry method allows accurate velocity measurements with broad band pulses

Use an interferometer to precondition broad band incoherent (white) radiation to illuminate object

Pass reflected radiation through a matching interferometer to detect phase difference induced by object motion

Measure time delay for range information

Preconditioning allows accurate measurement of phase shifts in broad band pulses with good range resolution 


\section{Schematic of white light velocimetry system}

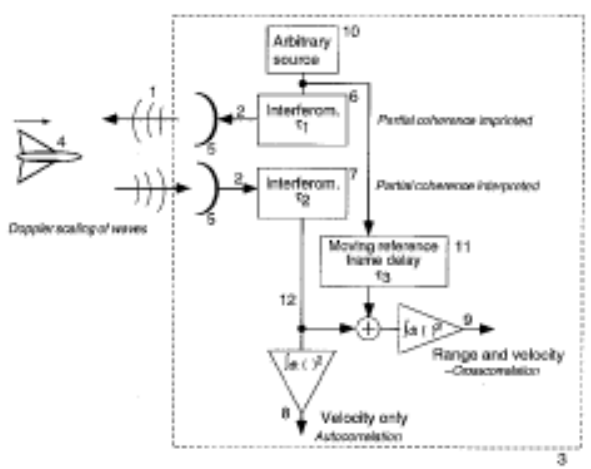

Goals of LDRD feasibility study

Determine accuracy of range and velocity estimation using

Erskine s original concept for an acoustic system

Apply concept to coherent broad band acoustic pulses

Determine accuracy limits for joint range and velocity estimation for this approach

Assess the generality of this concept through better understanding of the role of preconditioning 
Acoustic implementation of white light velocimetry concept

Transmit basic pulse $p(t)$ and time delayed version: $p(t)+p\left(t-t_{1}\right)$

Reflected pulse is $p,(t)=A[p(\mathrm{~g} t-\mathrm{t})+,p(\mathrm{~g} t-\mathrm{ht},-\mathrm{t}))$,$] ,$

$$
\mathrm{t}_{r}=\frac{2 r}{c}, \quad \mathrm{~h}=I+\frac{2 U}{c}, \quad \mathrm{~g}=\frac{I+U / c}{I-U / c}
$$

Add delayed version of reflected pulse to itself: $p_{r}(t)+p_{r}\left(t-t_{2}\right)$

Calculate intensity as function of second delay

$$
I\left(\mathrm{t}_{2}\right)=\left[p_{2}(t)+p_{2}\left(t-\mathrm{t}_{2}\right)\right]^{2} d t
$$

Intensity peaks when $\mathrm{t}_{2}=\mathbf{h} \mathbf{t}_{1}$, then $U=\frac{1}{2} c\left(\mathrm{t}_{2}-\mathrm{t}_{l}\right) / \mathrm{t}_{i}$

\section{Experimental setup}
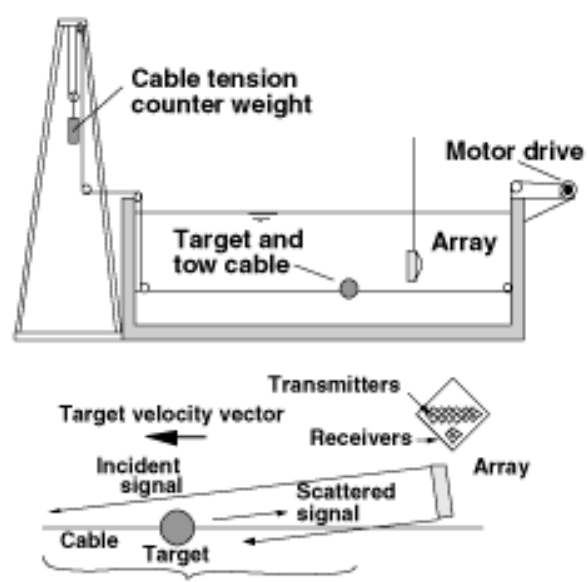

Measurement window 


\section{Experimental cases}

Tested three Gaussian pulses with half widths $0.706,1.18$, and

$3.53 \mathrm{mB}$, and three $150 \mathrm{kHz}$ tone bursts of length 3,7 , and 11 cycles

Towed air filled sphere at six speeds: $0.0,0.56,0.70,0.84,0.98$, and $1.1 \mathrm{~m} / \mathrm{s}$

Pulses generated by arbitrary waveform generator with $\mathrm{Dt}=0.1 \mathrm{~ms}$

Initial delay $t_{1}$ is $250 \mathrm{~ms}$

From condition $\left|\mathrm{t}_{2}=\mathrm{t}_{1}\right|>\mathrm{Dr}$ we have $U>\frac{c \mathrm{Dt}}{2 \mathrm{t}_{f}}=30 \mathrm{~cm} / \mathrm{s}$

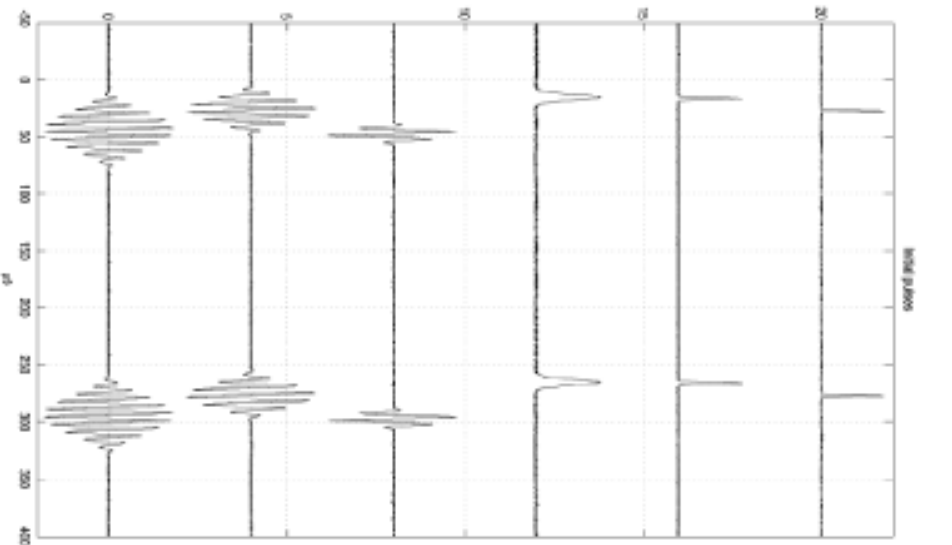


S I G
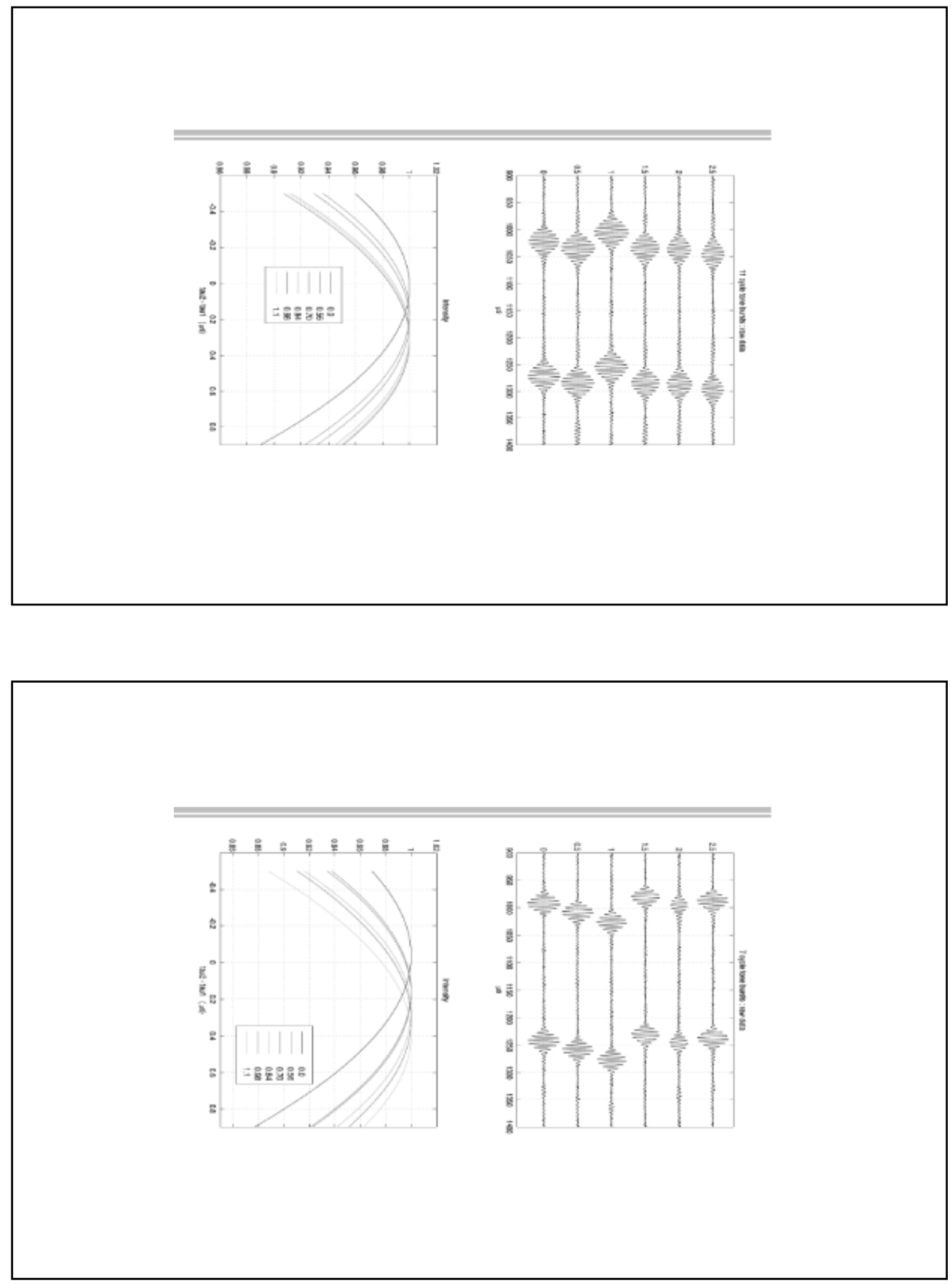

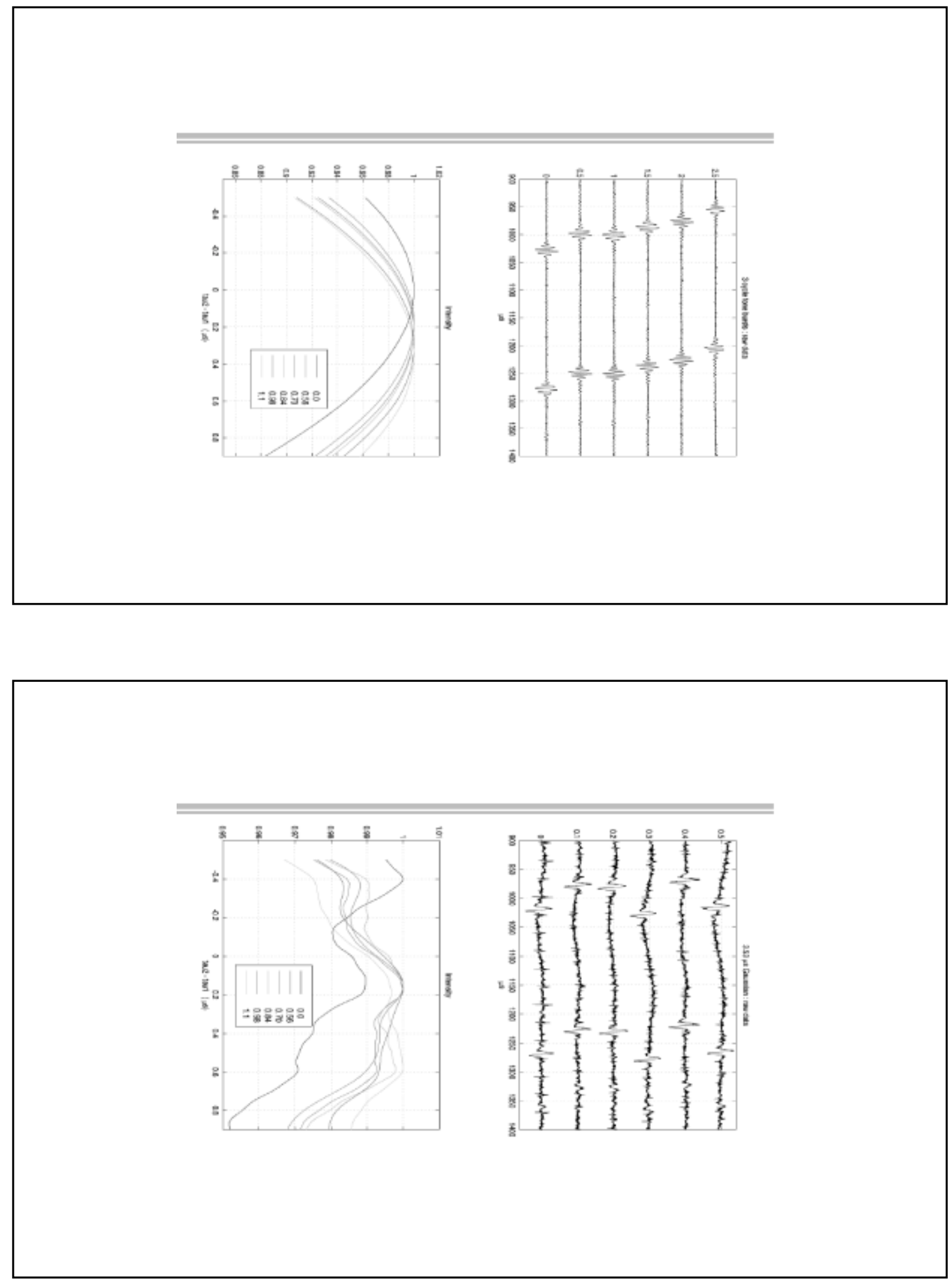

SIGNAL AND IMAGING SCIENCES WORKSHOP 1999 
S I G N A L P R O C E S S I N G
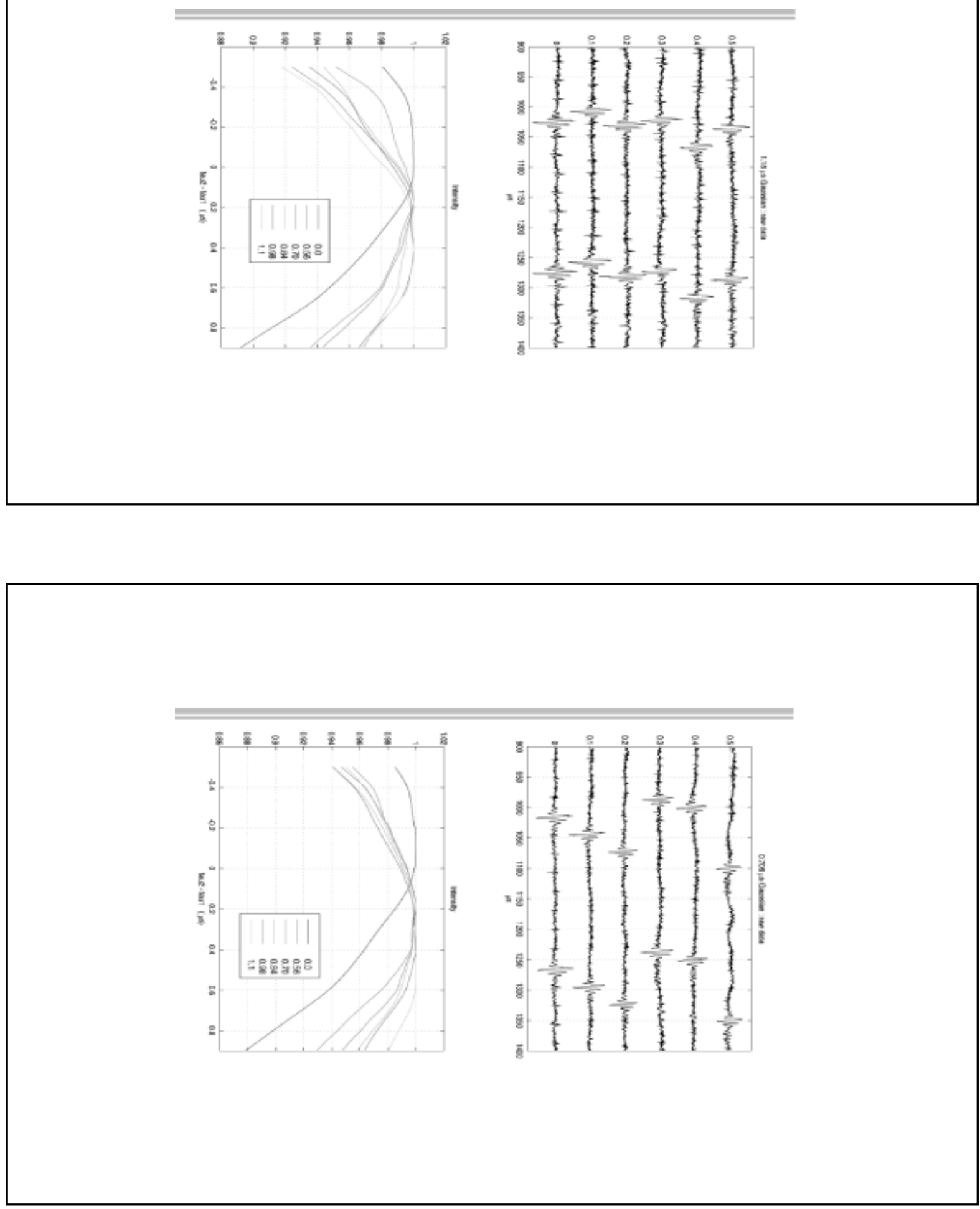
Velocity estimates agree with exact values within experimental error

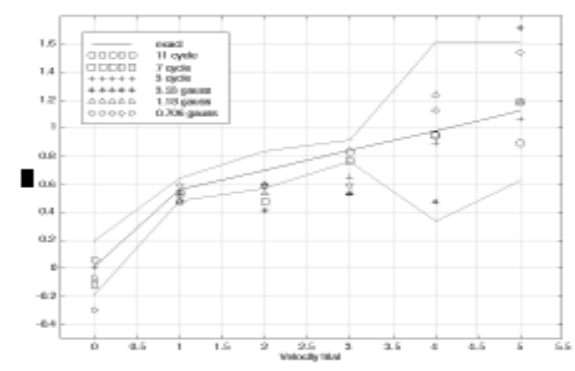

Range estimates from cross correlations are reasonable

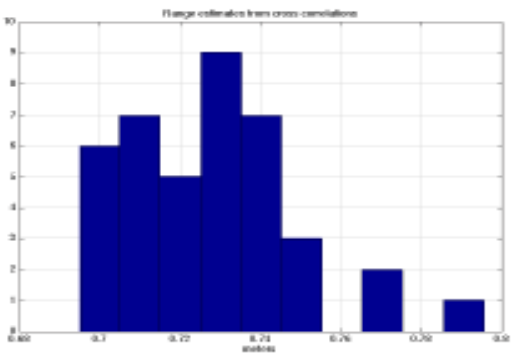




\section{Conclusions}

Erskine s velocimetry technique has been demonstrated for coherent broad band and narrow band acoustic pulses

Accuracy is increased with longer initial delay times or finer time discretization $(\mathrm{Dt} / \mathrm{t},<<1)$

\section{Grating Lobe Reduction in Large Element, 2-Dimensional Phased Arrays}

Jan-Ulco A. Kluiwstra 


\title{
Denoising Data using Wavelet Based Methods
}

\author{
UCRL-VG-136075 \\ by Chuck Baldwin and Chandrika Kamath
}

An important and highly researched problem in many fields of physics, engineering, and mathematics is the extraction of true data from data that has been contaminated by additive Gaussian noise. The use of wavelet coefficient thresholding has been an active topic of research for de-noising signal and image data and has proven to be a useful technique in certain applications. We will motivate this talk by presenting an application of data mining using astronomical data-sets and describe how the underlying noise gives rise to difficulties in feature extraction. We will then present some of the research into data denoising through the use of orthogonal wavelet transforms: including applicable noise models, issues of wavelet basis selection and level of multi-resolution decomposition, thresholding policies and the various techniques for threshold selection. Finally, we will conclude by presenting some results of the wavelet de-noising process on the previously mentioned data-set and what we have learned from the work.

Key Words: Wavelet Transform, Image Denoising, Thresholding

\section{Denoising Data Using Wavelet Based Methods}

\author{
Chuck Baldwin, Chandrika Kamath \\ Center for Applied Scientific Computing \\ Lawrence Livermore National Laboratory
}




\section{The FIRST survey}

- We are working with scientists from the FIRST (Faint Images of the Radio Sky at Twenty-cm) project to automatically detect radio-emitting galaxies with particular morphologies. The data are from radio interferometric observations at the NRAO Very Large Array (VLA) in Socorro NM. Currently, around 400,000 sources contained in approximately $100 \mathrm{~GB}$ of data have been collected.

- The data exhibits Gaussian noise as well as artifacts from the reconstruction of the signal. It is important to extract as much of the true data as possible since the processed data will be used in later pattern recognition techniques. The success of those techniques will depend on the quality of the denoised data.

\section{The FIRST data}

Some of the details of the observations are :

- Over 10,000 square degrees of the North Galactic Cap.

- 3-minute snapshots are acquired covering a hexagonal grid.

- The data are edited, self-calibrated, mapped, and CLEANed using AIPS.

- A final atlas of maps is produced by coadding the twelve images adjacent to each pointing center.

Some of the details of the data are :

- Maps have 1: 8" pixels with a resolution of 5" and a typical rms of 0: $15 \mathrm{mJy}$.

- The noise in the coadded maps varies by only $15 \%$ from the best to the worst places in the maps, except in the vicinity of bright sources ( $>100 \mathrm{mJy}$ ) where sidelobes can lead to an increased noise level.

- At the $1 \mathrm{mJy}$ source detection threshold, there are $\sim 90$ sources per square degree, $\sim 35 \%$ of which have resolved structure on scales from 230". 


\section{Introduction}

Suppose we have $N$ true data values $\left\{f\left(\chi_{i}\right), i=1, \ldots, N\right\}$ which are collected with noise introduced

$$
\begin{aligned}
Y_{i} & =f\left(\chi_{i}\right)+\epsilon_{i} \\
& =f_{i}+\epsilon_{i}
\end{aligned}
$$

where

$$
\epsilon \sim N\left(0, \sigma^{2}\right)
$$

is the noise component.

The goal is to reduce the effects of the noise so that the true data values can be recovered. As can be expected, the "quality" of the recovered true data will be directly related to how well the noise can be estimated and modeled, i.e. reasonable SNR (Signal to Noise Ratio).

\section{The Wavelet Decomposition I}

Let $W$ represent the orthogonal matrix associated with the orthonormal wavelet system of choice. Suppose

$$
Y=\left(Y_{1}, Y_{2}, \ldots, Y_{N}\right)^{T}
$$

is a vector associated with the noisy data values and set

$$
w=W Y=W(f+\epsilon) .
$$

The "empirical" wavelet coefficients

$$
w=\left(w_{0,0}, w_{1,0}, w_{1,1}, \ldots, \theta_{J I, 2}^{J-1}{ }_{-1}^{T}\right.
$$

are a noisy estimate for the true wavelet coefficients

$$
\boldsymbol{\theta}=W f=\left(\boldsymbol{\theta}_{0,0}, \boldsymbol{\theta}_{1, \theta}, \boldsymbol{\theta}_{1,1}, \ldots, \boldsymbol{\theta}_{J-1,2}^{J-1}{ }_{-1}\right)^{T}
$$

converging at the parametric rate

$$
w_{j, k}-\theta_{j, k} \sim N\left(0, \sigma^{2}\right) .
$$




\section{The Wavelet Decomposition II}

The relationship between the coefficients is expressed as

$$
w_{j, k}=\theta_{j, k}+z_{j, k},
$$

where the set of $z_{j, k}$ are a set of (unobservable) independent $N\left(0, \sigma^{2}\right)$ random variables.

Each empirical wavelet coefficient consists of a certain amount of noise but relatively few contain significant signal. This leads to a heuristic of how the true data could be extracted from the noise.

1. Separate coefficients which contain significant signal from those that contain mostly noise.

2. Once the significant coefficients are found, attempt to remove the noise from the empirical coefficients by dropping (or shrinking) the coefficients that contain mostly noise.

\section{Wavelet Thresholding I}

These two points are justified by noting :

- Large "true" coefficients $\boldsymbol{\theta}_{j, k}$ will typically have large corresponding empirical coefficients $w_{j, k}$.

- The "noisy" coefficients $z_{j, k}$ contribute little to the "true" coefficients and can typically be ignored.

This heuristic yields the idea of thresholding the empirical coefficients. 


\section{Wavelet Thresholding II}

Thresholding the empirical coefficients yields an estimate of the true coefficients

$$
\tilde{\boldsymbol{\theta}}=\left(\tilde{\boldsymbol{\theta}}_{0,0}, \tilde{\theta}_{1,0}, \tilde{\theta}_{1,1}, \ldots, \tilde{\theta}_{J-1,2}^{J-1}{ }_{-1}\right)^{T}
$$

and an approximation to the true data

$$
\tilde{f}=W^{T} \tilde{\boldsymbol{\theta}}
$$

The thresholding estimator of the true coefficients is written as

$$
\tilde{\theta}_{j, k}=\sigma \delta_{\lambda k} \frac{\left(w_{j, k}\right)}{\sigma} .
$$

The non-linear threshold function $\delta_{\lambda_{k}}()$ has the task of making the determination of the significance of a empirical coefficient as well as modifying the coefficient itself.

\section{Estimating the Variance}

The variance $\sigma^{2}$ reguired in the threshold estimator is usually not known so an estimate is required. The median of absolute deviation (MAD) of a semple is given as

$\operatorname{MAD}\left(Z_{1}, Z_{2}, \ldots, Z_{N}\right)=\operatorname{median}_{k}\left(\mid Z_{k} \quad \operatorname{median}_{k}\left(Z_{k}\right)\right)$.

This quantity applied to the highest level coefficients is usually used as an estimate $\sigma^{2}$.

$$
\hat{\sigma}^{2}=\frac{\operatorname{MAD}\left(w_{J-1, k}\right)}{0.6745}
$$




\section{Denoising Process}

The denoising process is given by the following procedure

1. Compute the wavelet transform $w-\mathcal{W} Y$.

2. Estimate the variance $\sigma^{2}$ by $\hat{\sigma}^{2}$.

3. Apply a non-linear threshold function $\delta_{\lambda_{k}}()$ to the empirical coefficients $\hat{\theta}_{j k}=\sigma \delta_{\lambda_{k}}\left(\begin{array}{c}w_{j k} \\ \sigma\end{array}\right)$.

4. Compute the inverse wavelet transform $\bar{f}=\mathcal{W}^{T} \bar{\theta}$.

The success will depend on how well the threshold function ean find and remove the noise from the empirical coefficients.

\section{Thresholding Functions}

Sereral chaices for the thresbold function $\delta_{2}$ are given in the litenature.

The hard thresbolding function is given by

$$
\delta_{\lambda_{4}}(x)=\left\{\begin{array}{lll}
x & \text { if } & x \mid>\lambda_{6} \\
0 & \text { if } & x \mid \leq \lambda_{k}
\end{array}\right.
$$

The soft thresholding function is given by

$$
\delta_{\lambda_{k}}^{5}(x)=\left\{\begin{array}{cl}
x-\lambda_{k} & \text { if } x>\lambda_{k} \\
0 & \text { if }|x| \leq \lambda_{k} \\
x+\lambda_{k} & \text { if } x<-\lambda
\end{array}\right.
$$

The semi-soft threshodding function is given by

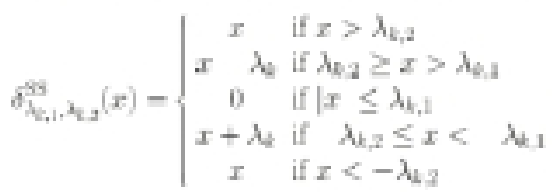



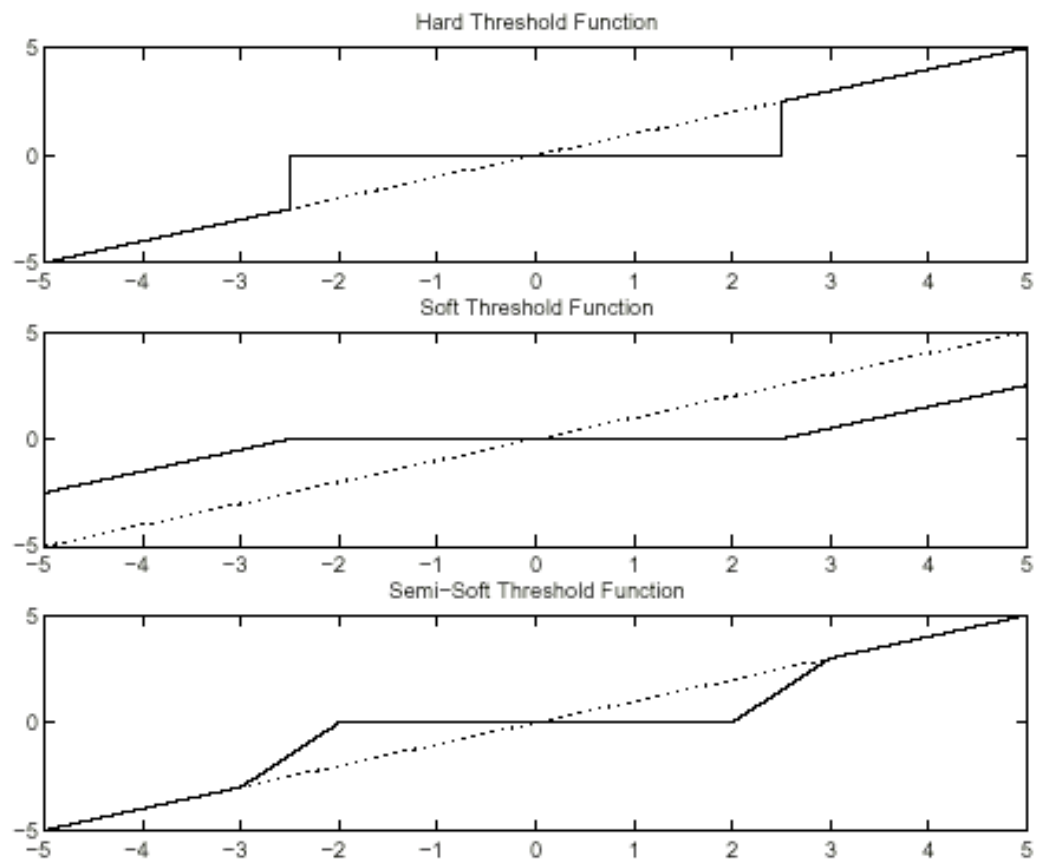

\section{Methods of Threshold Selection}

Many methodologies are in the literature on how to select thresholds :

- Manual thresholds

- Universal thresholds

- Mini-max thresholds, "RiskShrink"

- Steins Unbiased Risk Estimate, "SUREShrink"

- Cross validation

- Hypothesis testing ( $\chi^{2}$ or Brownian Sheet)

- Bayes rules 


\section{Global Thresholding I}

Global thresholding uses a single value of the threshold parameter and usually is applied to only one (or a few) level(s) of empirical wavelet coefficients.

$$
\lambda_{k}= \begin{cases}\lambda & \text { if } k \geq j \\ 0 & \text { if } k<j\end{cases}
$$

where $\bar{J}$ is the lowest leve] to which thresholding will be applied.

Two ideas for choosing the threshold parameter $\lambda$ are noted in the literature:

- universal thresholding

- minimax thresholding

\section{Global Thresholding II}

The universal thresholding parameter is

$$
\lambda=\sqrt{2 \log N} \sigma
$$

The universal threshold depends only on the sample size $N$. It is derived from probabilistic arguments.

The minimax thresholding parameter is a constant of the form

$$
\lambda=c(N) \sigma
$$

The minimax threshold depends on the sample size $N$ and the type of threshold function used. It is derived from minimizing the constant term in an upper bound of $L^{2}$ risk. 


\section{Level Dependent Thresholding}

The idea of universal thresholding can be extended to level dependent thresholding by the following

$$
\lambda_{k}=\sqrt{2 \log N} \sigma_{k}
$$

where $\sigma_{k}$ is the variance of the noise in the level $k$ coefficients. Using the previous MAD variance estimator this can be stated as

$$
\begin{gathered}
\lambda_{k}=\sqrt{2 \log N} \operatorname{MAD}\left(w_{J-1, k}\right) \\
0.6745
\end{gathered}
$$

\section{SURE Thresholding I}

The least $L^{2}$ risk of an estimator $\bar{f}$ to $f$ is given by

$$
\left.\mathrm{R}(\hat{f}, f)=\mathbf{E} \mid \frac{1}{n} \sum_{i=1}^{n}\left(\bar{f}_{i}-f_{i}\right)^{2}\right\} .
$$

By Parseval's identity this yields

$$
\mathrm{R}(\bar{f}, f) \propto \mathbf{E}\left[\frac{1}{n} \sum_{i} \sum_{j}\left(\bar{\theta}_{j, k}-\theta_{j, k}\right)^{2}\right] .
$$

So that minimizing $l^{2}$ risk in the wavelet domain will atttomatically minimize $L^{2}$ risk in the original domain.

This leads to the use of Steins Unbiased Risk Estimator (SURE) for threshold selection. 


\section{SURE Thresholding II}

The $f^{2}$ risk can be estimated unbuasedly (for the soft thresh

old function) for a given $t$ and $x=\left(x_{1}, \ldots, x_{d}\right)^{t}$ by

$\operatorname{SURE}(t, x)=-d+2\left(\operatorname{card}\left(\left\{k:\left|x_{k}\right|>t\right\}\right)+\sum_{k=1}^{d} \min \left(\left|x_{k}\right|, t\right)\right.$,

where card ( $)$ denotes the cardinality of a set. The thresbold

knd $\lambda$ is set so as to minimize the estimate of risk for given data $\left(x_{1}, \ldots, x_{d}\right)^{T}$.

$$
\lambda=\arg \min _{t \geq 0} \operatorname{SURE}(t, x)
$$

The SUFE eriteria is usually used to sedect a threshold for each level of coefficients

$$
\lambda_{k}=\underset{E \geq 0}{\arg \min } \operatorname{SURE}\left(t,\left\{w_{j, k}\right\}_{j}\right)
$$

\section{Measuring Quality I}

In order to quantify how much noise has been removed from the true data and how much the true data has been altered a few measures are needed.

Given a collection of data $y=\left(y_{1}, \ldots, y_{N}\right)^{T}$ the energy or $l^{2}$ norm is defined as

$$
\|y\|_{2}^{2}=\sum_{i=1}^{N}\left|y_{i}\right|^{2} .
$$

and gives an idea as to the "content" of the data. 


\section{Measuring Quality II}

Given a discrete probability $p=\left(p_{1}, \ldots, p_{M}\right)^{T}$ the $\log$ entropy is defined as

$$
E^{L}(p)=-\sum_{i=1}^{M}\left\{\begin{array}{cl}
\log \left(p_{i}^{2}\right) & \text { if } p_{i} \neq 0 \\
0 & \text { if } p_{i}=0
\end{array}\right.
$$

while the Shannon entropy is defined as

$$
E^{S}(p)=-\sum_{i=1}^{M}\left\{\begin{array}{cc}
p_{i}^{2} \log \left(p_{i}^{2}\right) & \text { if } p_{i} \neq 0 \\
0 & \text { if } p_{i}=0
\end{array}\right.
$$

The log entropy weights the occurrence of low probability events less (or high probability events less with $1-p$ ). The Shannon entropy weights the occurrence of low and high probability events less.

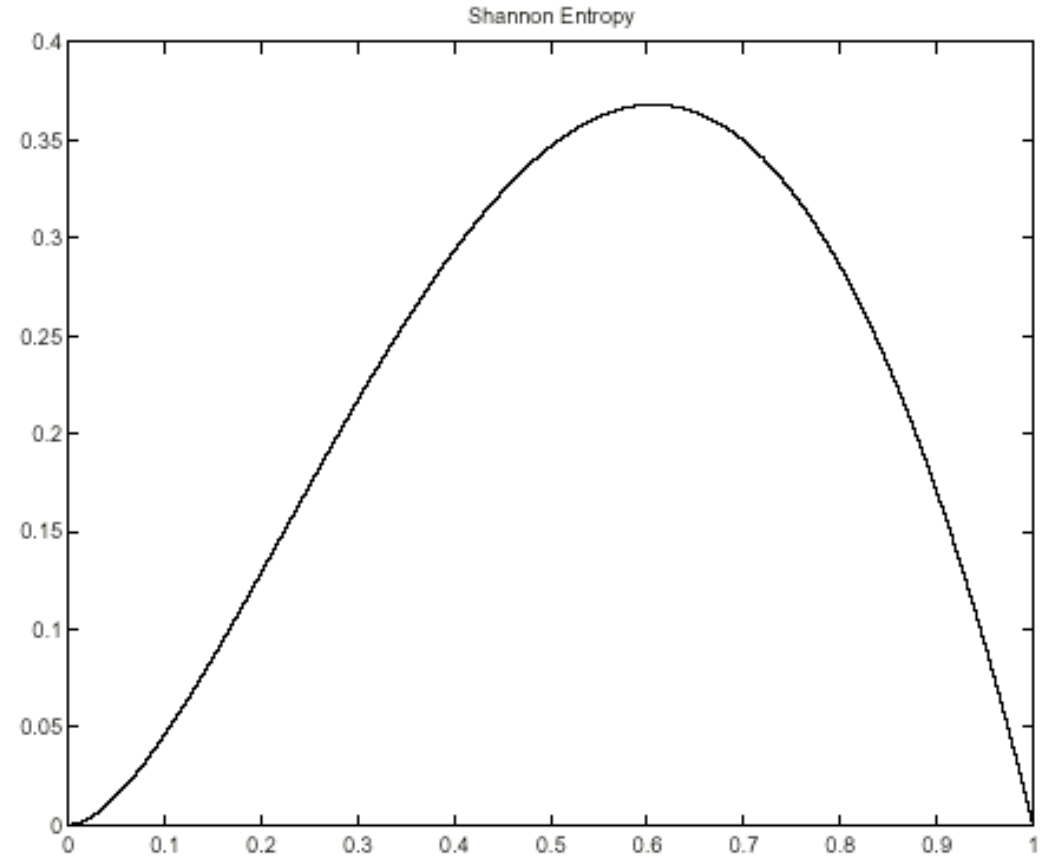



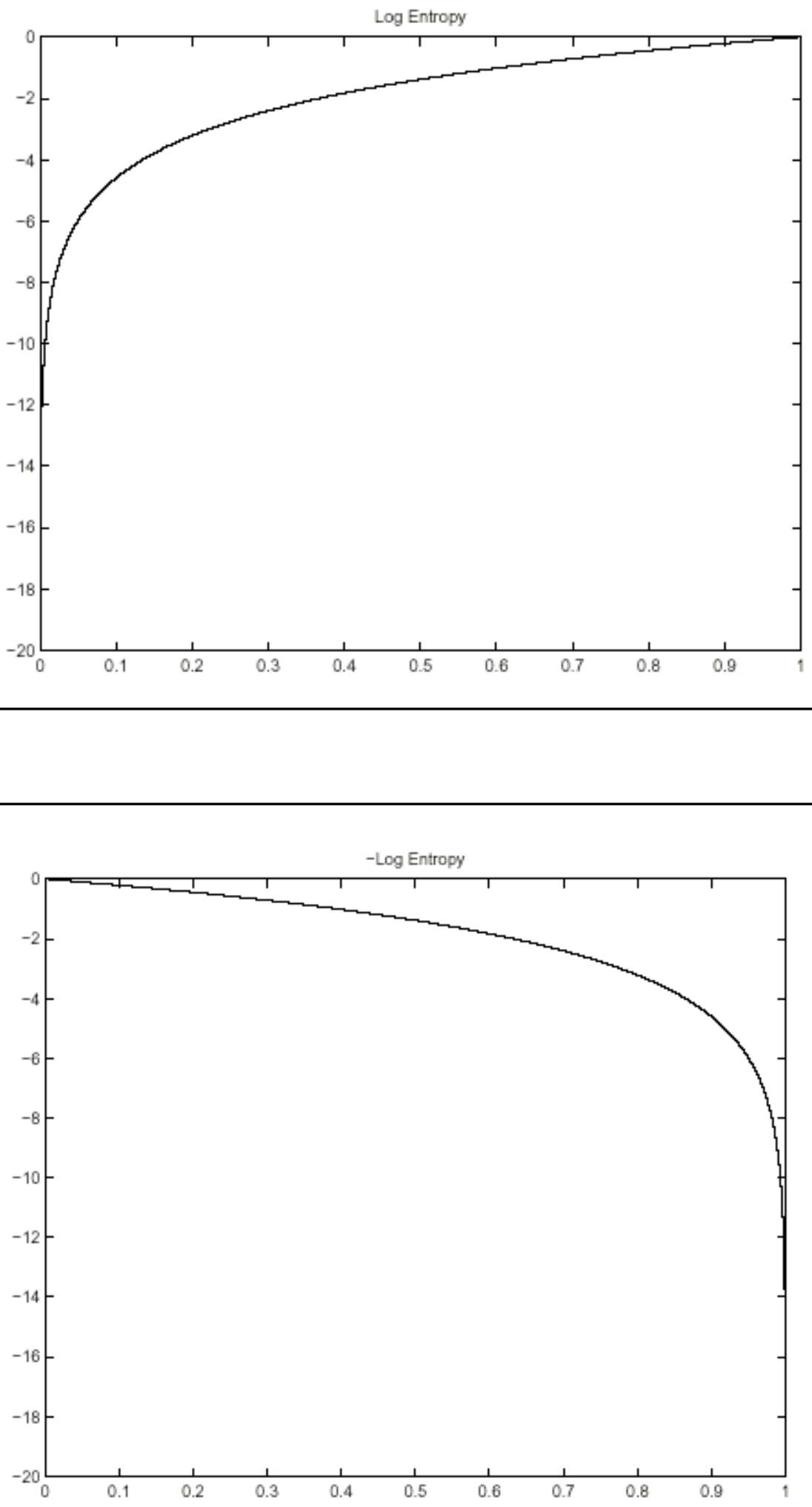
S I G N A L P R O C E S S I N G

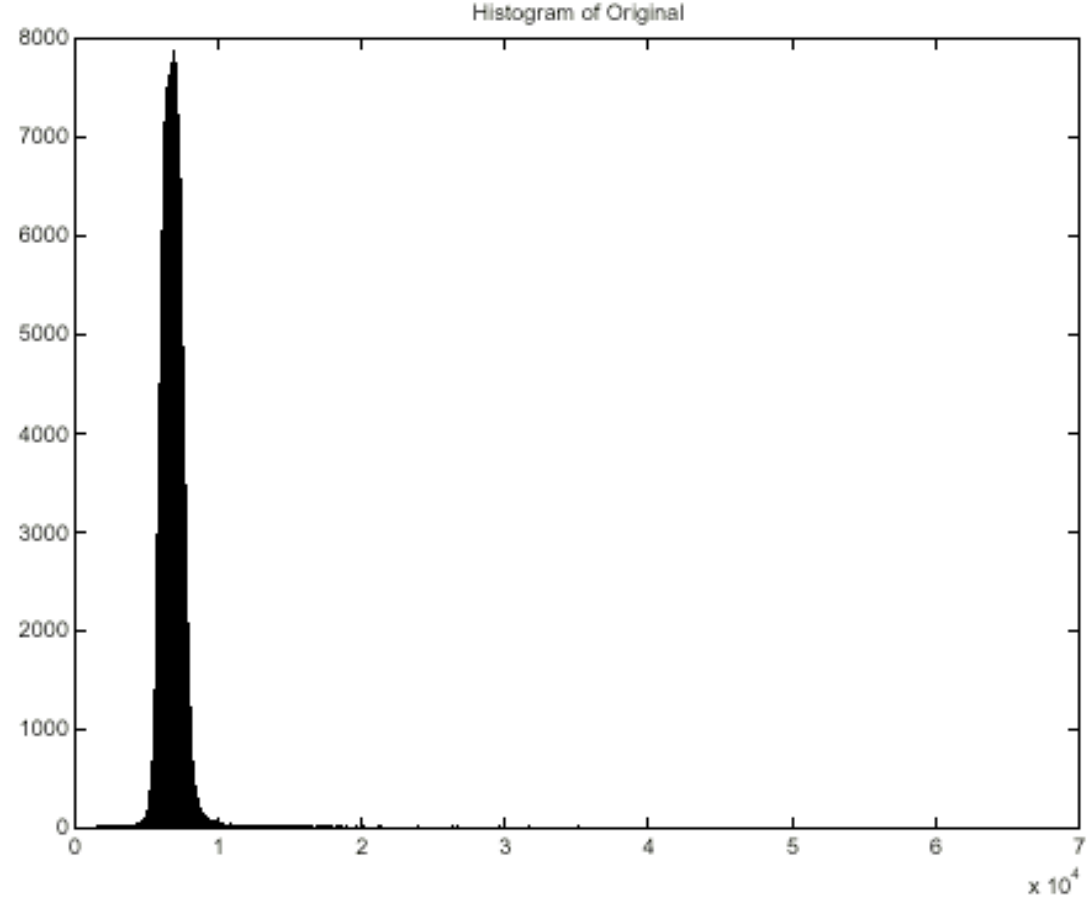

\section{Some Results}

\begin{tabular}{|c|c|c|c|c|}
\hline Dra & $f^{2}$ Energ & Fearuop $/ 7$ & 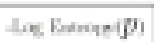 & Lug Eaingil $1-p$ \\
\hline ans & 1.mencentas & Legsta-11 & 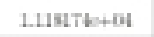 & $21200 x+60$ \\
\hline Wimas & 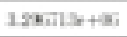 & $2 \operatorname{sex} i k n$ & answetout & $2 \operatorname{mos} 6 x+6 n$ \\
\hline $\mathrm{Hen}$ & acoutsetos & 8.S114tiess & 3.213100+04 & 2 povesteter \\
\hline atdi & isounistai & $6 \sqrt{2} \cos -6$ & $1: 1650+66$ & $2 \operatorname{mos} 4 i s+00$ \\
\hline dxal & 20ursictos & aร̃arses & 1. vouastos & 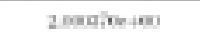 \\
\hline$a(4)$ & $12 m+16+a i$ & $70005 \pi-6]$ & $1.5160 \times 6+06$ & $2 \sin 6 x+40$ \\
\hline dx & 1. & $6.10=200 \times 3$ & 1.202ancte6 & 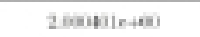 \\
\hline$a(6)$ & Istoustos & 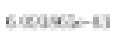 & $1.9 \times 350+06$ & 2 iscusisten \\
\hline mosti & 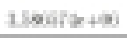 & ixastises & 1. Matowith & $2 \sin x+x+60$ \\
\hline ल(ख) & $2.450 b+a$ & cesusen & 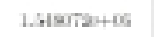 & $2.000124+60$ \\
\hline keit.al & 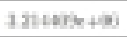 & 7.Sestibes & a exacestou & $2 \sec 6120+60$ \\
\hline Wa|1,5| & 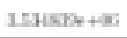 & ceosicen & Sanvintatod & 2.00015isters \\
\hline ka1221 & $3 \sec 2 x+a$ & ₹11314kes & 1. $8 x+7 b+16$ & 2 monterten \\
\hline$N=\mid 2,11$ & $12416 n+\infty$ & 6.11) & $1.03100+06$ & $2.00003 x+63$ \\
\hline Nelzsi & 3.4xestetas & exientacks & 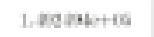 & 2 escusietes \\
\hline Na|1,II & imaLstas & $2000200-10$ & $14120+60$ & 2. morutite+es \\
\hline Neisu| & 3.48516etos & 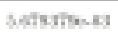 & 1. Thertiontes & 2 mostetes \\
\hline 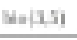 & 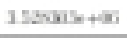 & cocarise- & $1.65 a 50+66$ & $2 \cos x 316+0$ \\
\hline Neital & $13001 \times+10 y$ & carezibus & 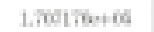 & $2 \operatorname{mos} 970+00$ \\
\hline
\end{tabular}


S I G N A L P R O C E S S I N G
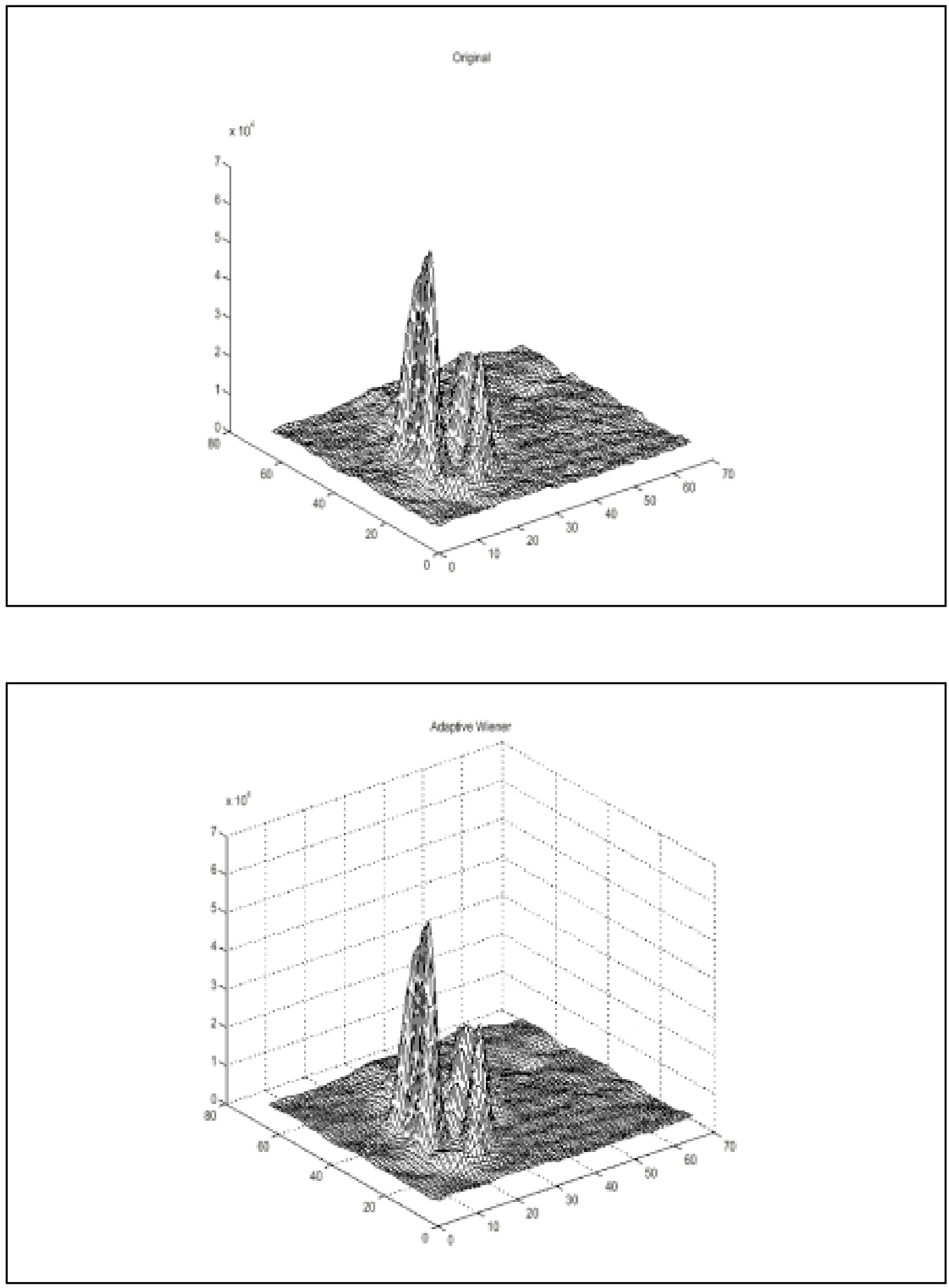

S I G N A L $\quad$ P R O C E S S I N G
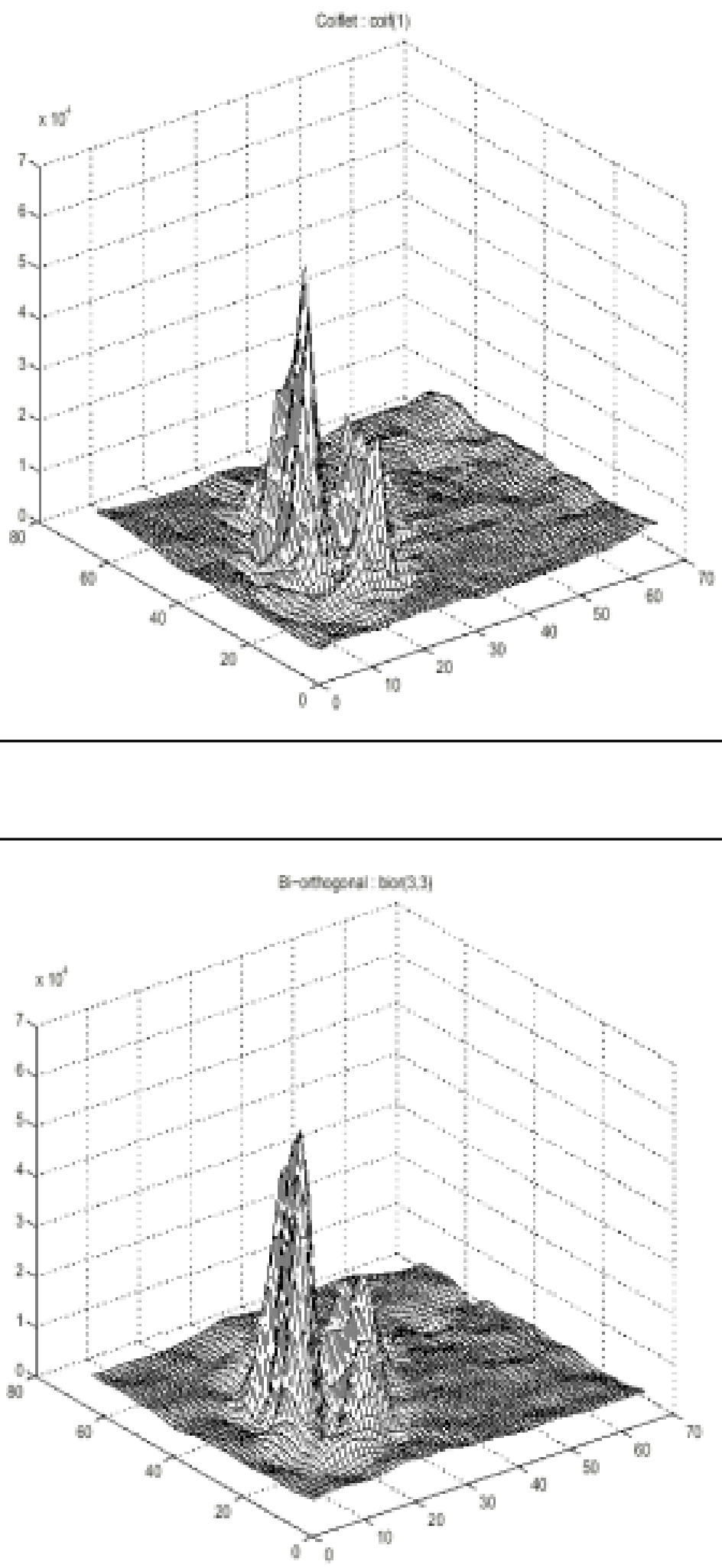


\section{More Results}

\begin{tabular}{|c||c|c|cc|}
\hline Data & $l^{2}$ Energy & Entropy(p) & Mean & Std. Dev. \\
\hline \hline orig & $3.559270 \mathrm{e}+06$ & $1.682872 \mathrm{e}-01$ & $6.847672 \mathrm{e}+03$ & $1.198133 \mathrm{e}+03$ \\
\hline Expert & $2.612073 \mathrm{e}+06$ & $6.747421 \mathrm{e}-03$ & $4.952996 \mathrm{e}+03$ & $1.222798 \mathrm{e}+03$ \\
\hline SURE & $2.880112 \mathrm{e}+06$ & $6.296412 \mathrm{e}-03$ & $5.485141 \mathrm{e}+03$ & $1.247522 \mathrm{e}+03$ \\
\hline Sqt2Log & $3.554193 \mathrm{e}+06$ & $1.841299 \mathrm{e}-02$ & $6.837687 \mathrm{e}+03$ & $1.197662 \mathrm{e}+03$ \\
\hline Mini-Max & $3.555799 \mathrm{e}+06$ & $2.473489 \mathrm{e}-02$ & $6.840845 \mathrm{e}+03$ & $1.197819 \mathrm{e}+03$ \\
\hline
\end{tabular}

\section{Observations}

Good Points :

- Wavelet Denoising is an effective methodology for removing (Gaussian) noise.

- Many varieties of thresholding functions and selection techniques exist.

- Most of the techniques for threshokling are computational efficient and parallel.

Bad Points :

- Not suitable for all applications, not a black box denoiser.

- Difficult to understand how threshold selection effects denoising.

- Many of the newer threshold selection techniques need to be coded. 


\section{The Sapphire Team: Supporting a multi-disciplinary endeavor}

- Chandrika Kamath (Project Lead)

- Chuck Baldwin

- Erick Cantu-Paz

- Imola Fodor

- Roy Kamimura

- Nu Ai Tang

http://www.1lnl.gov/casc/sapphire 


\title{
Hematoma Detection Using MIR
}

\author{
CASIS Workshop \\ John Chang, $\mathrm{PhD}$ \\ David Scott
}

$-1 M T P P^{\text {nosous }}$

\section{The Need for the Hematoma Detector}

\section{Problem:}

- Hemorrhage of major cerebral vesiculature due to head trauma

- Life threat due to pressure on the brain and brain stem

- Closed head injuries cannot be positively diagnosed without CT

- Delay presentation of clear symptoms with associated increase in mortality

\section{Solution:}

- Exploit LLNL Micropower Impulse Radar (MIR) technology for early screening

- Provide in field diagnosis and triage Neurosurgical procedure preparation

Govern patient evacuation method

- Improve overall patient management

- Life saving diagnostic tool

YMTP 


\section{Available Technology}

\section{About Micropower Impulse Radar}

- low power - runs on $9 \mathrm{~V}$. battery

- small and very lightweight

- low cost - MIR electronics can be mass produced for

- microwave radiation penetrates body relatively well

- safe - order of magnitude lower power than a cellular phone

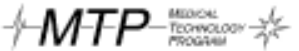

\section{Radar Specifications}

- Frequency range: 1.5 to $4 \mathrm{GHz}$ ( $3 \mathrm{GHz}$ center)

- Peak power: 1 Watt

- (at $10 \mathrm{~cm}$, no gain: 800 microwatt $/ \mathrm{cm}^{2}$ )

- Average power: 400 microwatt

- (at $10 \mathrm{~cm}$, no gain: 0.3 microwatt $/ \mathrm{cm}^{2}$ )

- Pulse repetition rate: $2 \times 10^{6}$ pulses $/ \mathrm{sec}$

- Pulse duration: $2 \times 10^{-10} \sec (200 \mathrm{ps})$

- Energy per pulse: $2 \times 10^{-10}$ Joules

tMTP 


\section{Radar Specification Exposure Measurements (continued)}

- Max antenna gain: 10

- Measurement distance from antenna: $10 \mathrm{~cm}^{2}$

- Average power deposited: $0.003 \mathrm{~mW} / \mathrm{cm}^{2}$

- Peak power deposited: $8 \mathrm{~mW} / \mathrm{cm}^{2}$

- Peak energy per pulse: 0.0000016 micro $\mathrm{J} / \mathrm{cm}^{2}$

$\left(1.6 \mathrm{pJ} / \mathrm{cm}^{2}\right)$

$-1-M T P P$

\section{How a "Rangefinder" Works}

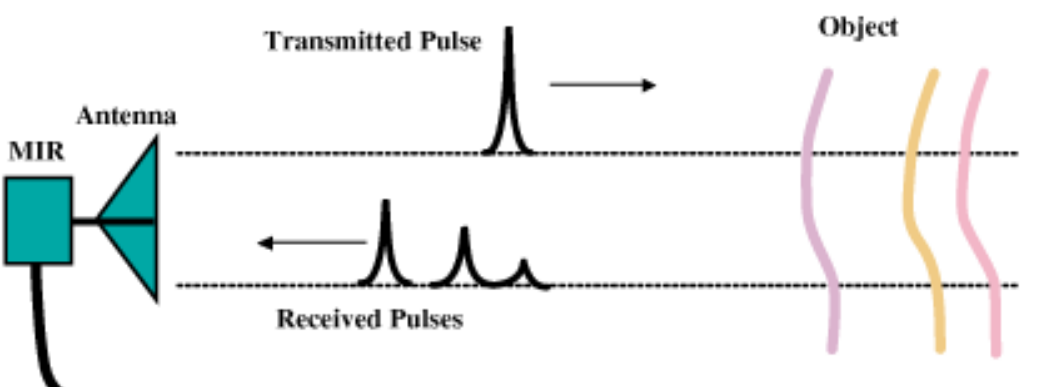

MIR fast pulse technology samples reflected pulses in time - similar to other pulse-echo techniques such as ultrasound

1 MTP 
S I G N A L P R O C E S S I N G

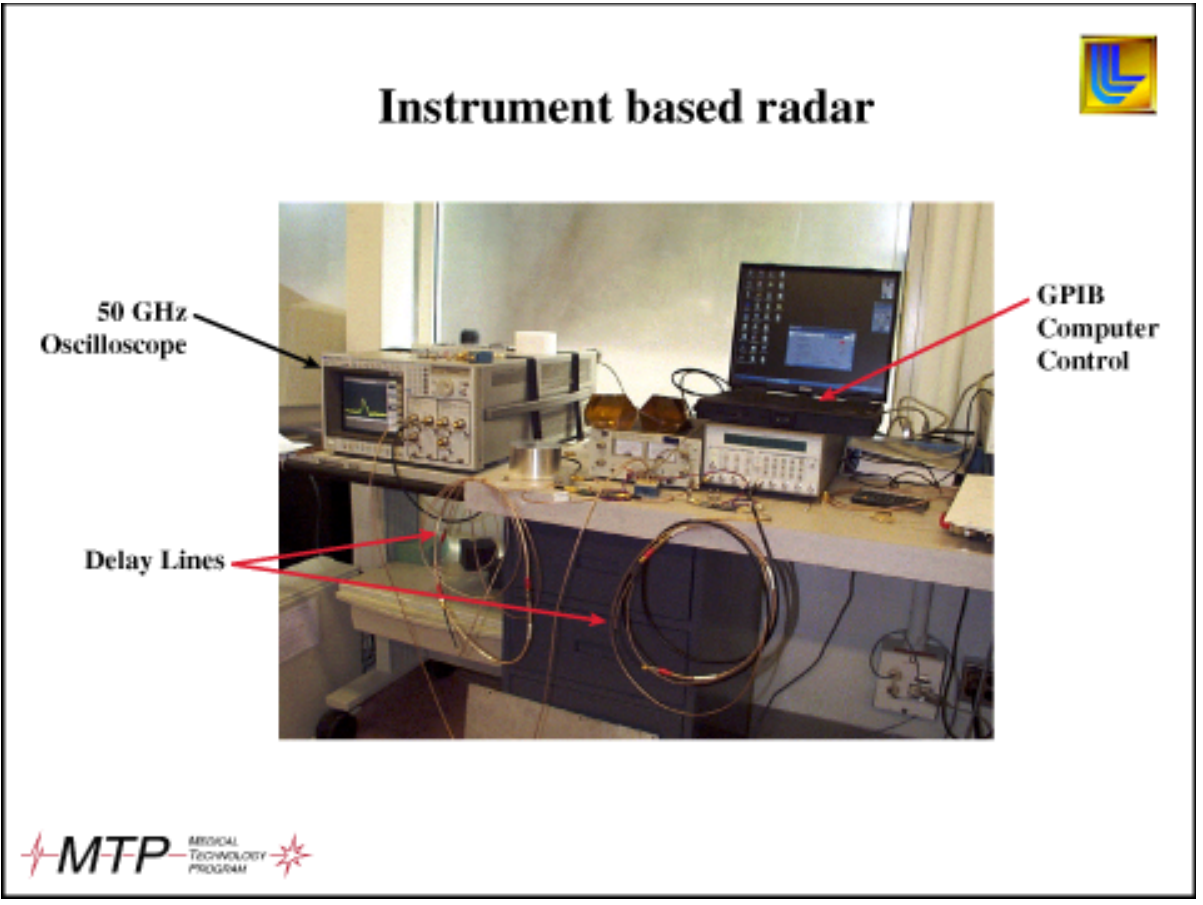

\section{The Hematoma Detector Records Returned Pulses from Dielectric Interfaces in the Head}

CT slice image of a patient with a large epi-dural hematoma

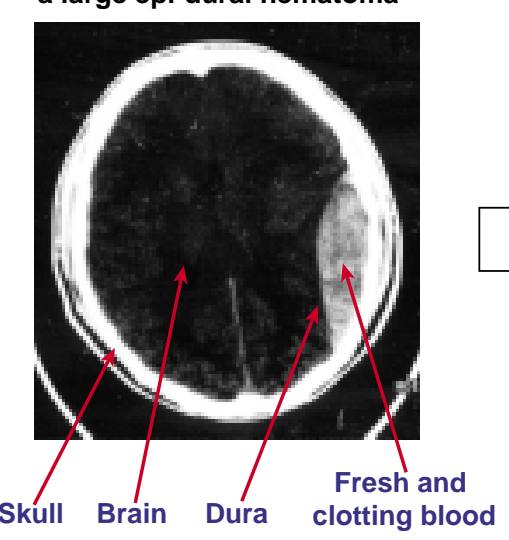

$4 M T P-\frac{1}{4}$
Region with hematoma:

Third interface will

produce an additional

Microwave

pulses in

returned pulse later in time

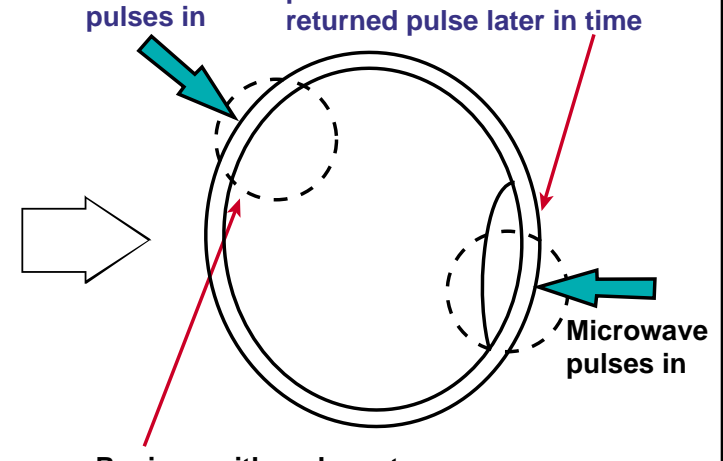

Regions with no hematoma:

Two interfaces will produce

two returned pulses close

together in time 


\section{Results}

- Numerical Model:

- Rectangular stratified slabs

- Patch antenna: coplanar rectangular, spiral, point source feed, dipole

- Transmitter/receiver distance is $30 \mathrm{~mm}$

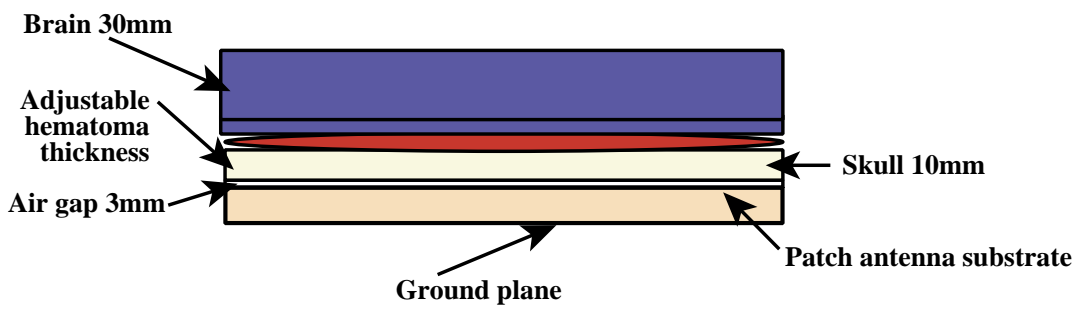

MTP

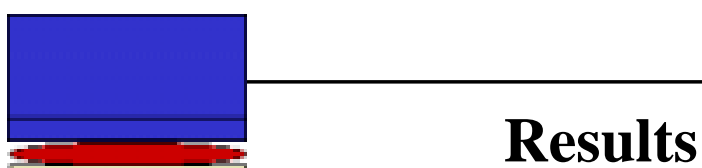

Effect of hematoma presence

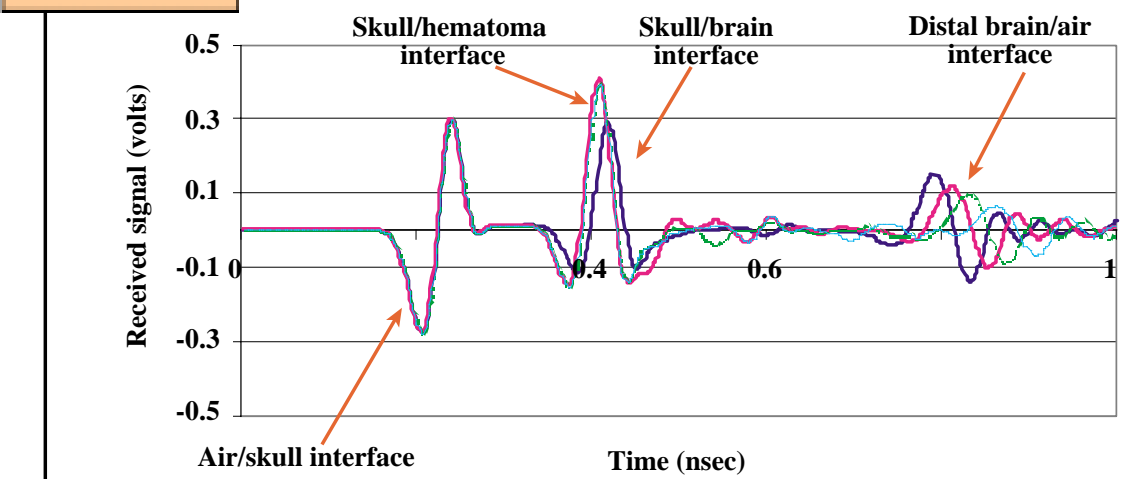

\begin{tabular}{|ll|}
\hline Baseline & 0.5 hemotoma \\
$----2 \mathrm{~mm}$ hemotoma & $5 \mathrm{~mm}$ hemotoma
\end{tabular}

HMTP $=-\frac{1}{4}$ 
S I G N A L P R O C E S S I N G
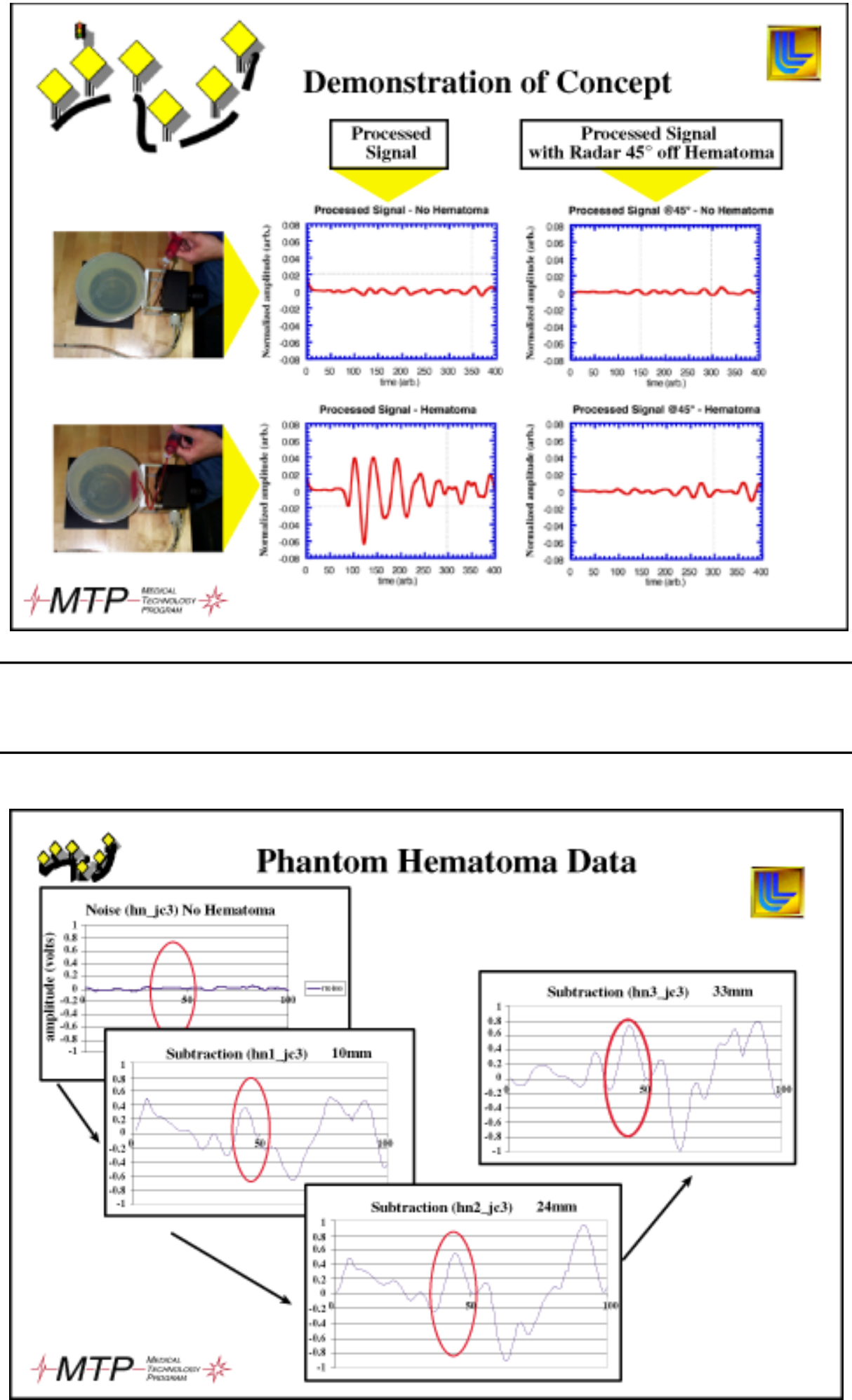
S I G N A L P R O C E S S I N G

\section{Porcine brain experiment}

\section{$-1-M T P$ -}

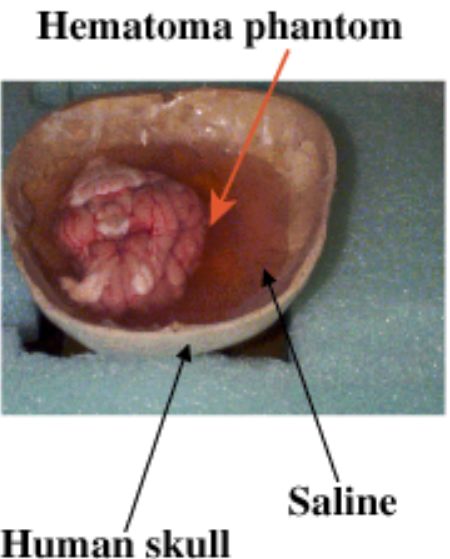

\section{Results}

Point45 (phantom-hn_jc)

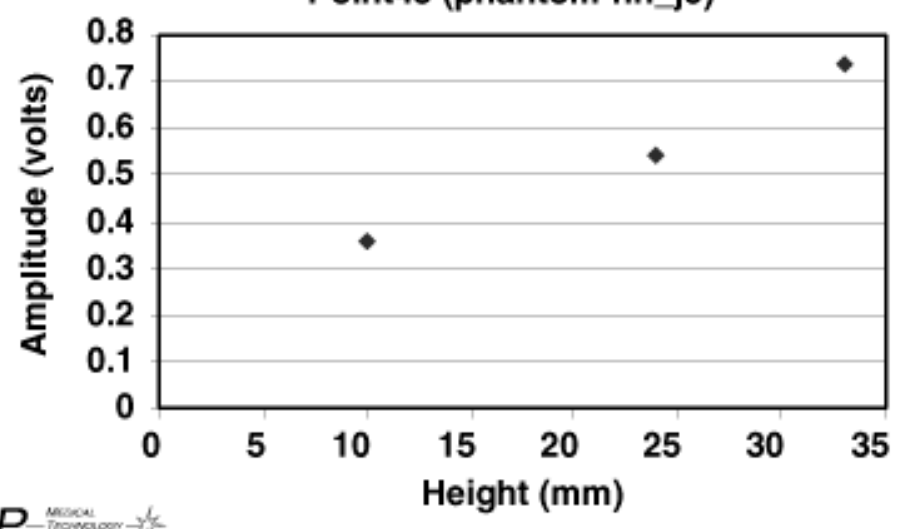

fMTP Height (mm) 


\section{Imaging and Detection

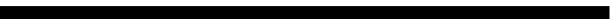




\section{Towards Automated Optical Evaluation of Protein Crystals}

UCRL 991112

Pat Fitch, Brent Segelke, Bernhard Rupp

Biology and Biotechnology Research Program

Lawrence Livermore National Laboratory

Preliminary results of our research towards developing an automated optical evaluation of protein crystals will be discussed. Several image processing approaches and algorithms are in the early stage of development and will be presented. Our algorithm development is performed on a desktop computer using MATLAB. The value of protein crystallization and the motivation for automating this part of the crystallization process is described below. If successful, a robust algorithm will allow more cost-effective high throughput protein crystallization to be implemented.

In the past few years there has been a tremendous increase in DNA sequence information available. In order to identify and understand the function of a gene, however, far more information than DNA sequence is required. Structural information about the protein that the DNA codes can provide significant information about function as well as insight into potential biochemical interactions (e.g., inhibitors), that can be used to modify function.

X-ray diffraction measurements of protein crystals can provide excellent structural information with resolution to about an Angstrom. The formation of protein crystals involves an educated search process for appropriate experimental conditions [1]. Each of the different samples (in some cases the same protein, but different experimental conditions) is monitored roughly 30 times before crystallization is complete. This monitoring is currently accomplished with a light microscope and visual evaluation. The images are given a quality score [2] to assist in determining if the crystallization process should continue, be aborted or modified, and/or fed-back into the crystal growth conditions. As automation, sample tracking and high throughput protein expression systems are integrated, the optical evaluation of the intermediate protein crystals becomes a bottleneck to high throughput. For instance, a robot can prepare 1,000 protein samples a day; each sample is imaged about 30 times before completion in roughly two months. If the robot keeps the pipeline full, this requires on average 1 / 2 an image each day for each sample. So at two months there would be 60,000 protein samples in process requiring 30,000 images to be viewed and evaluated each day. This is more than an image every second assuming an 8 hour workday.

[1] http: / / www-structure.llnl.gov / Web site for the structural biology group in the Biology and Biotechnology Research Program that includes protein crystal information and a nice tutorial.

[2] C.W. Carter Jr. and C.W. Carter, Protein crystallization using incomplete factorial experiments, Biological Chemistry, Vol. 254, No. 23, Dec. 10, 1979, pp. 12219-12223. 


\title{
In-Situ Sensing of Nucleation and Crystal Growth of $\mathrm{Cd}_{1-x} \mathrm{ZnxTe}$
}

\section{B. William Choi}

The nucleation and growth of $\mathrm{Cd}_{1-x}$ ZnxTe crystals in a multi-zone vertical Bridgman growth furnace have been observed and measured using in-situ eddy current sensor techniques. A two-coil eddy current sensor measured coil impedance changes for multifrequency which were then interpreted using an electromagnetic finite element analysis. The sensor was used to characterize the initial melting of a charge and the subsequent nucleation of solid during solidification. Fully remelted in-situ compounded charges were exposed to significant melt superheating and were found to undergo large melt undercoolings (of up to $20^{\circ} \mathrm{C}$ ), spontaneous crystal nucleation and rapid solidification (velocities approaching $60 \mathrm{~mm} / \mathrm{h}$ which was more than ten times the furnace translation rate). Post growth metallography revealed that about $20 \mathrm{~mm}$ of polycrystalline solid was formed in this way before recalescence arrested the solidification interface. In partially remelted charges neither undercooling nor unstable growth were observed. These results indicate that eddy current sensors can be used to monitor critical aspects of the vertical Bridgman crystal growth of semiconducting materials and may simplify the implementation of seeded crystal growth concepts in this, and other, semiconductor crystal growth processes.

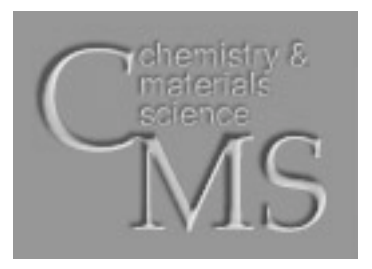

\section{Real_Time Signal Processing of the Nucleation and Early Vertical Bridgman Growth of CdZnTe}

\author{
by \\ ByoungWoo William Choi \\ Hayden N. G. Wadley
}




\section{Presented at CASIS'S}

Signal \& Imaging Sciences Workshop

UC@LLNL

Livermore, CA

November 12, 1999

SCHOOLOF

ENGINEERING

\& APPLIED SCIENCE

Intelligent Processing of Materials

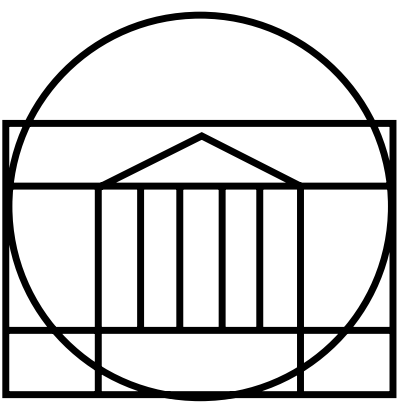

Dept. of Materials Science and Engineering

University of Virginia

\section{CdZnTe Ingot Grown by Vertical Bridgman Furnace JME 9035}

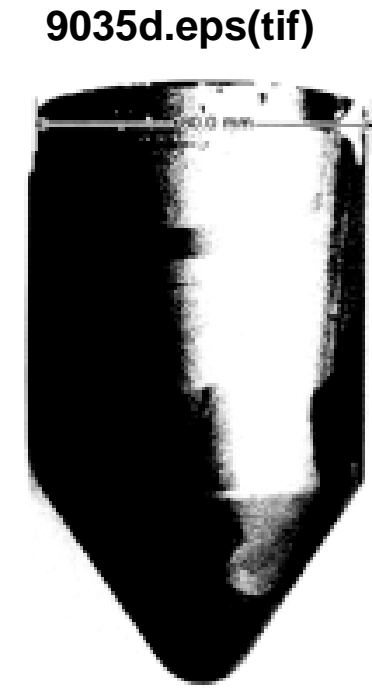

a) Boule Surface 9035e.eps(pcx)

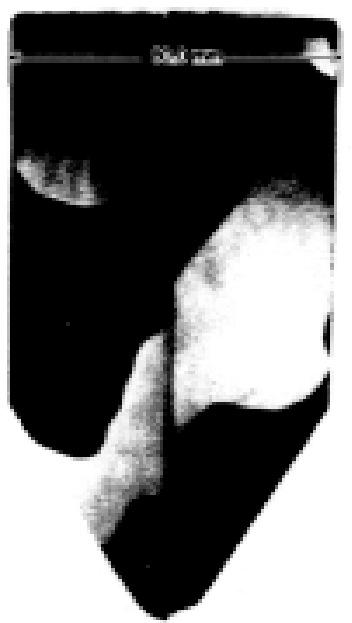

b) Longitudinal Plane 


\section{Schematic Diagram of 17 Zone Vertical Bridgman Furnace}

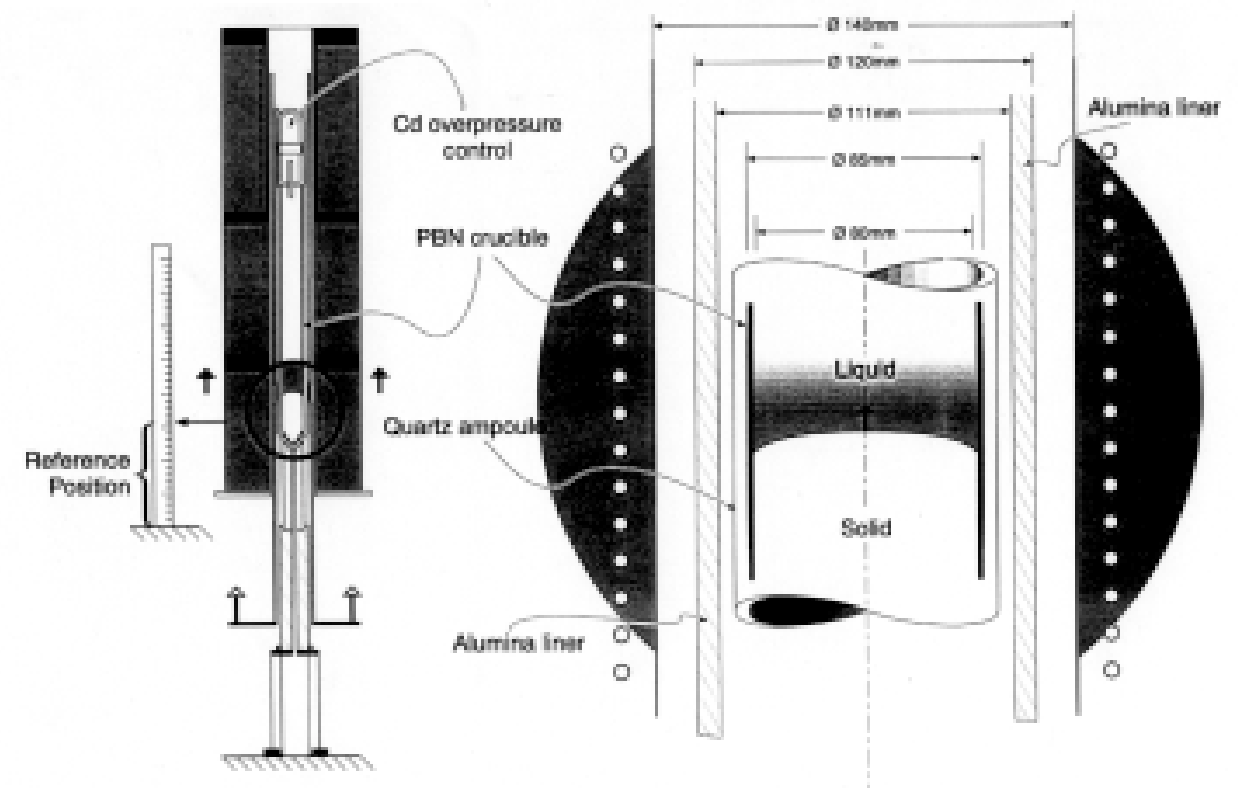

\section{CdZnTe Ingot Grown by Vertical Bridgman Furnace}

- Unseeded Directional Solidification

Cd: Te stoichiometric control by

Cd overpressure techniques

- Final Products

- Multigrain Ingot

- Significant Zn Macrosegregation (i.e. $0.02 \leq x \leq 0.07$ )

- Frequent Tellurium Precipitation

- High Dislocation Density etc.

The lowest stacking fault energy of all semiconducting compounds which crystallize in the sphalerite structure disappointingly low yield. 

I $M$ A G I $N$ -
A N D
D E

\section{CdZnTe Ingot Grown by Vertical Bridgman Furnace JME 9035}

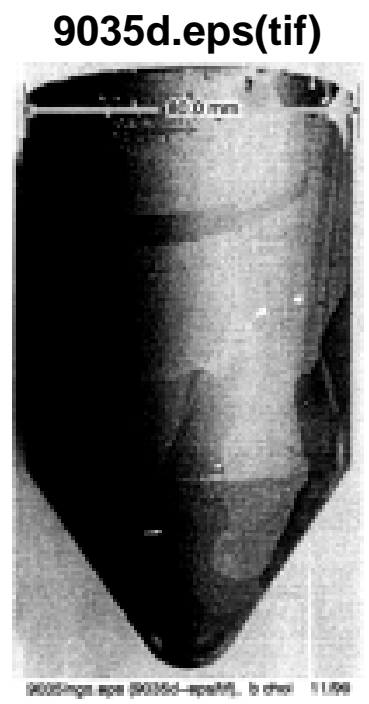

a) Boule Surface

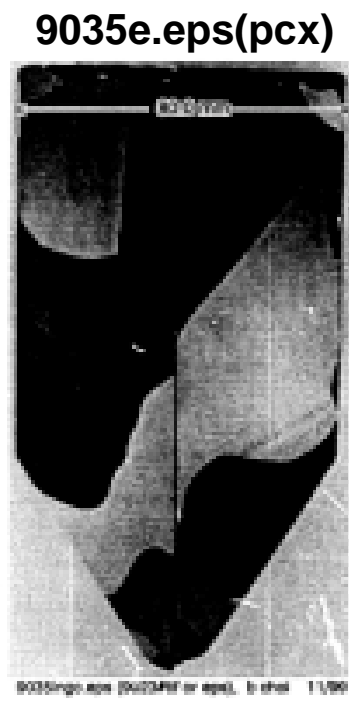

b) Longitudinal Plane

\section{CdZnTe Ingot Grown by Vertical Bridgman Furnace}

- What should we know to improve this growth technology?

- Melt Stoichiometry

- Superheating and Undercooling

- Nucleation/Growth

- Solidification Velocity

- Interface Shape

- Temperature Gradients

- Cooling Rate etc. 


\section{Circuit Diagram of Two Coil Impedance Measurement System}

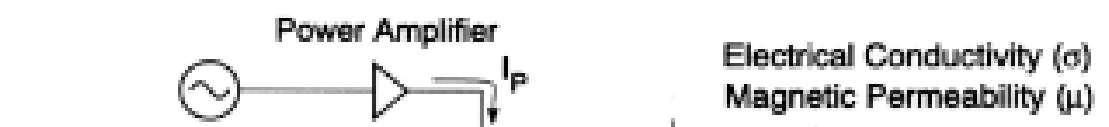

Variable frequency $(f)$ oscillator $(50 \mathrm{kHz}=5 \mathrm{MHz})$

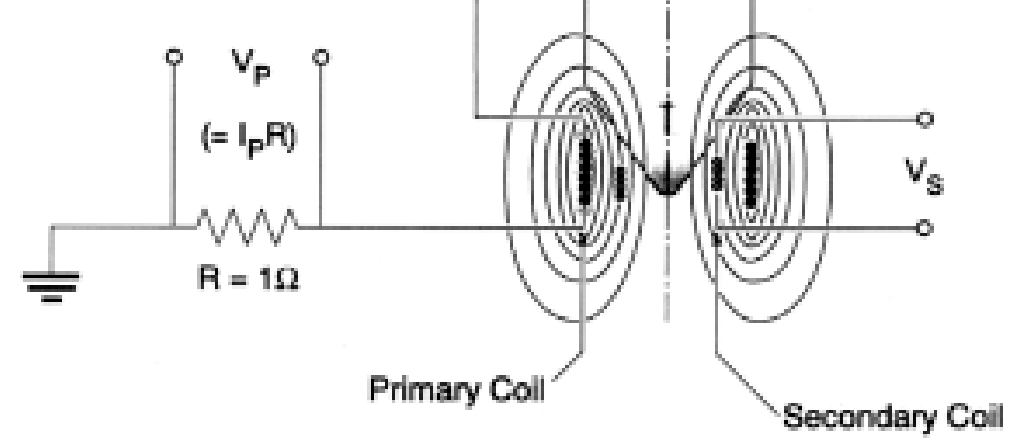

Measure:

Gain $(G)=V_{s} / V_{p}$ Phase $(\phi)=\phi_{s} / \phi_{p}$

\section{Calculate:}

Real $Z=G / G_{0} \sin \left(\phi-\phi_{0}\right)$

Imaginary $Z=G / G_{0} \cos \left(\phi-\phi_{0}\right)$

\section{Calculation of Electrical Conductivity from Eddy Current Data}

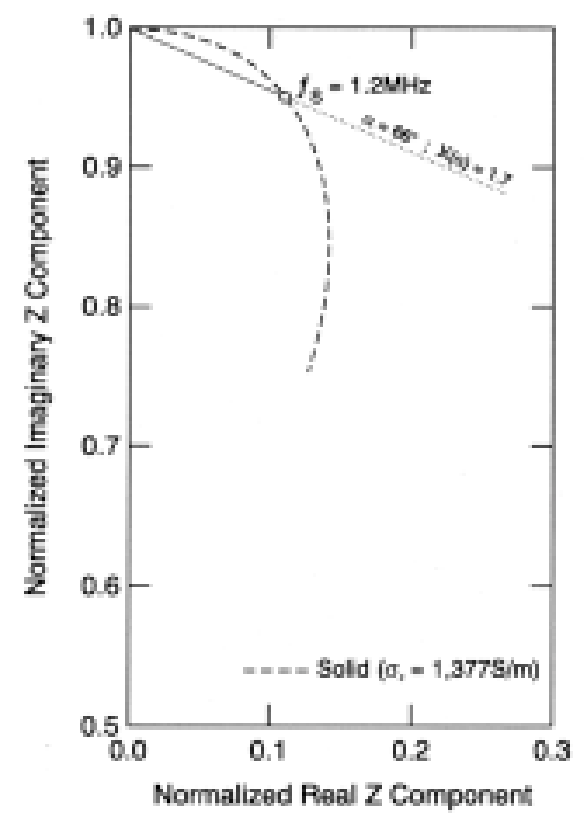




\section{Calculation of Electrical Conductivity from Eddy Current Data}

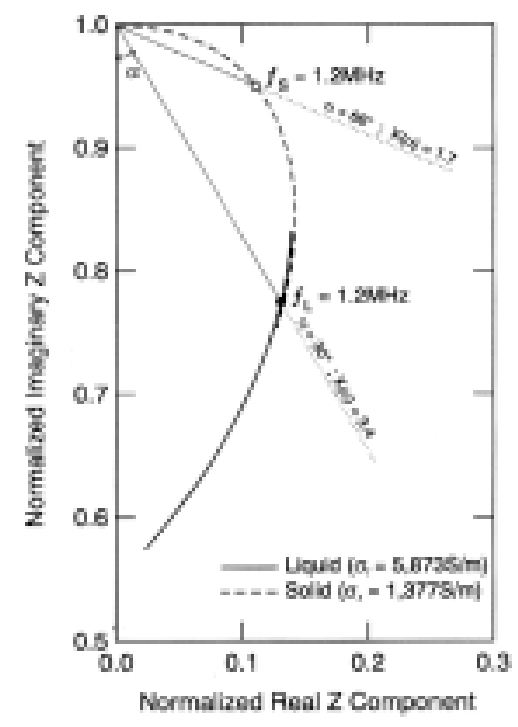

$$
\begin{aligned}
& 8 \cdot \sqrt{\frac{2}{\omega \mu \sigma}} \\
& x(\omega)=a \sqrt{2 \pi f \omega \sigma}
\end{aligned}
$$

where, $\quad \mathbf{R}=$ radius of sample $(\mathrm{m})$

$f=$ frequency $(\mathrm{Hz})$

$\mu=$ magnetic permeability $(\mathrm{H} / \mathrm{m})$

$\sigma=$ electrical conductivity $(\mathrm{S} / \mathrm{m})$

\section{Refernce Silicon Cylinder Properties measured witha 4-point probe.}

\begin{tabular}{|c|c|c|c|}
\hline Ingot \# & $\begin{array}{c}\text { Conductivity } \\
\text { (S/m) by } \\
\text { 4 point } \\
\text { probe }\end{array}$ & $\begin{array}{c}\text { Conductivity } \\
\text { (S/m) by } \\
\text { Eddy } \\
\text { Current }\end{array}$ & $\begin{array}{c}\text { Frequency } \\
\text { Range } \\
\text { (kHz) }\end{array}$ \\
\hline MN1-1861 & $1,471-1,149$ & $1,390 \pm 60$ & $800-1,200$ \\
\hline MP0-5792 & $6,667-4,000$ & $5,810 \pm 170$ & $300-700$ \\
\hline JME-31844 & $11,628-11,234$ & $11,700 \pm 170$ & $120-300$ \\
\hline
\end{tabular}


I M A G I N G A N D D E T E C T I O N

\section{FEM Geometry for Nucleation Sensor}
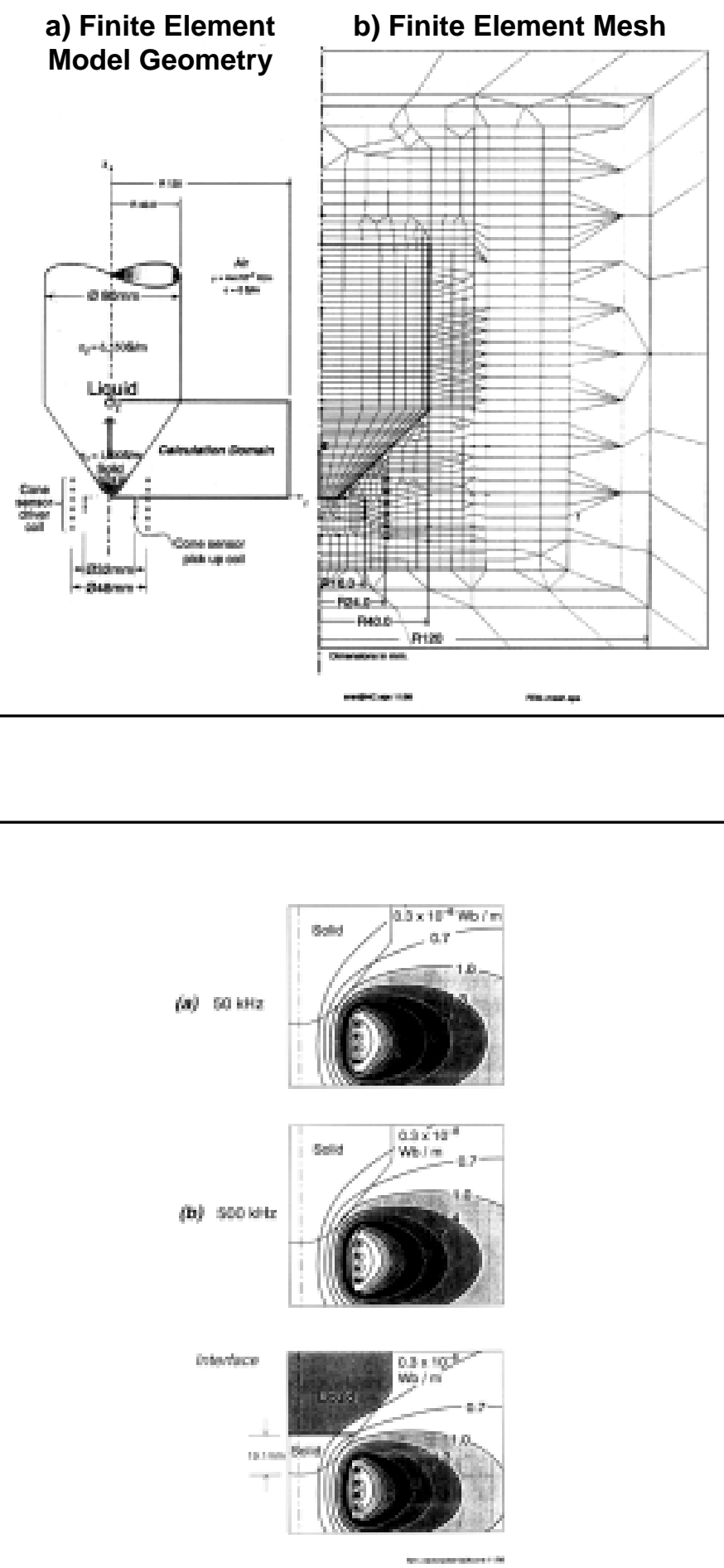


\section{Nucleation Sensor}

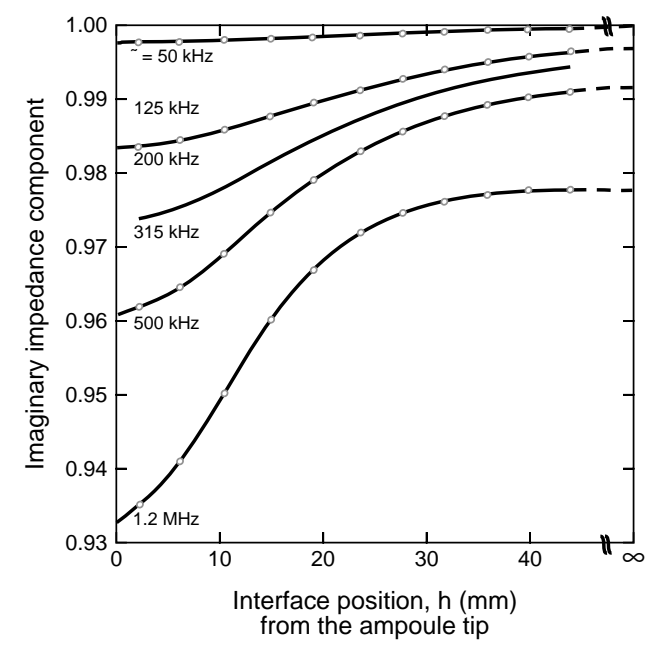

SCHOOL OF

ENGINEERING 命览

\& APPLIED SCIENCE

\section{A Series of Eddy Current Sensing Configurations}

a) 17 zone Furnace

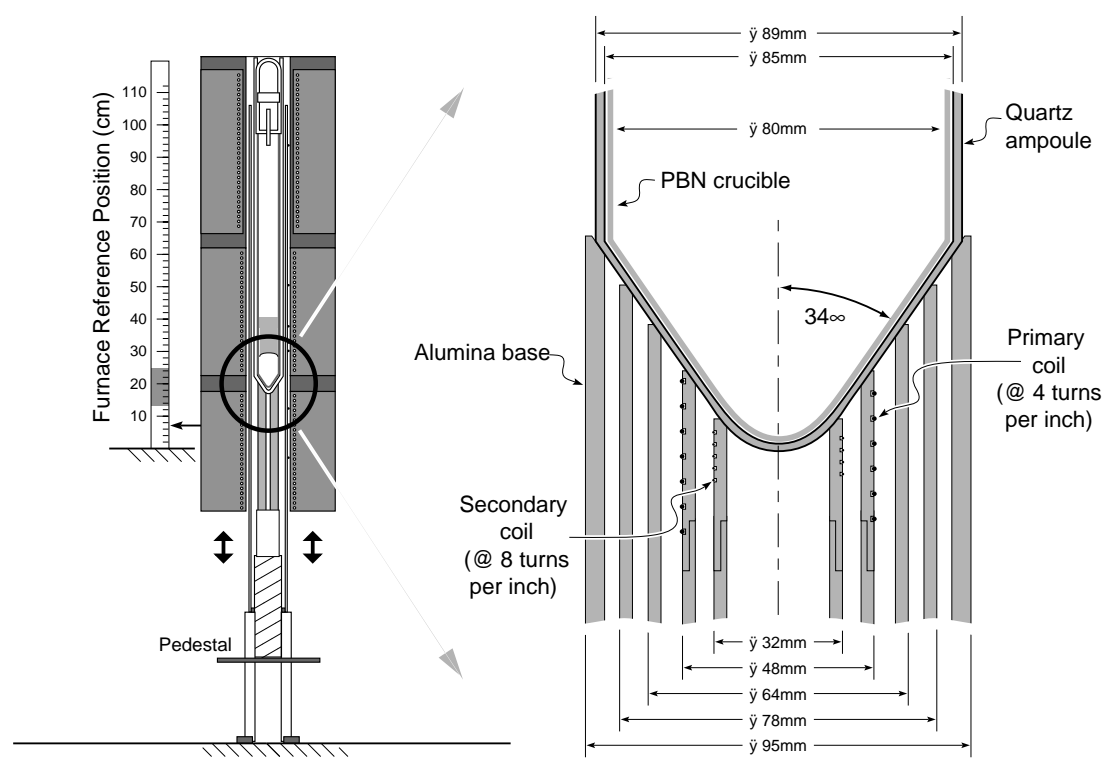



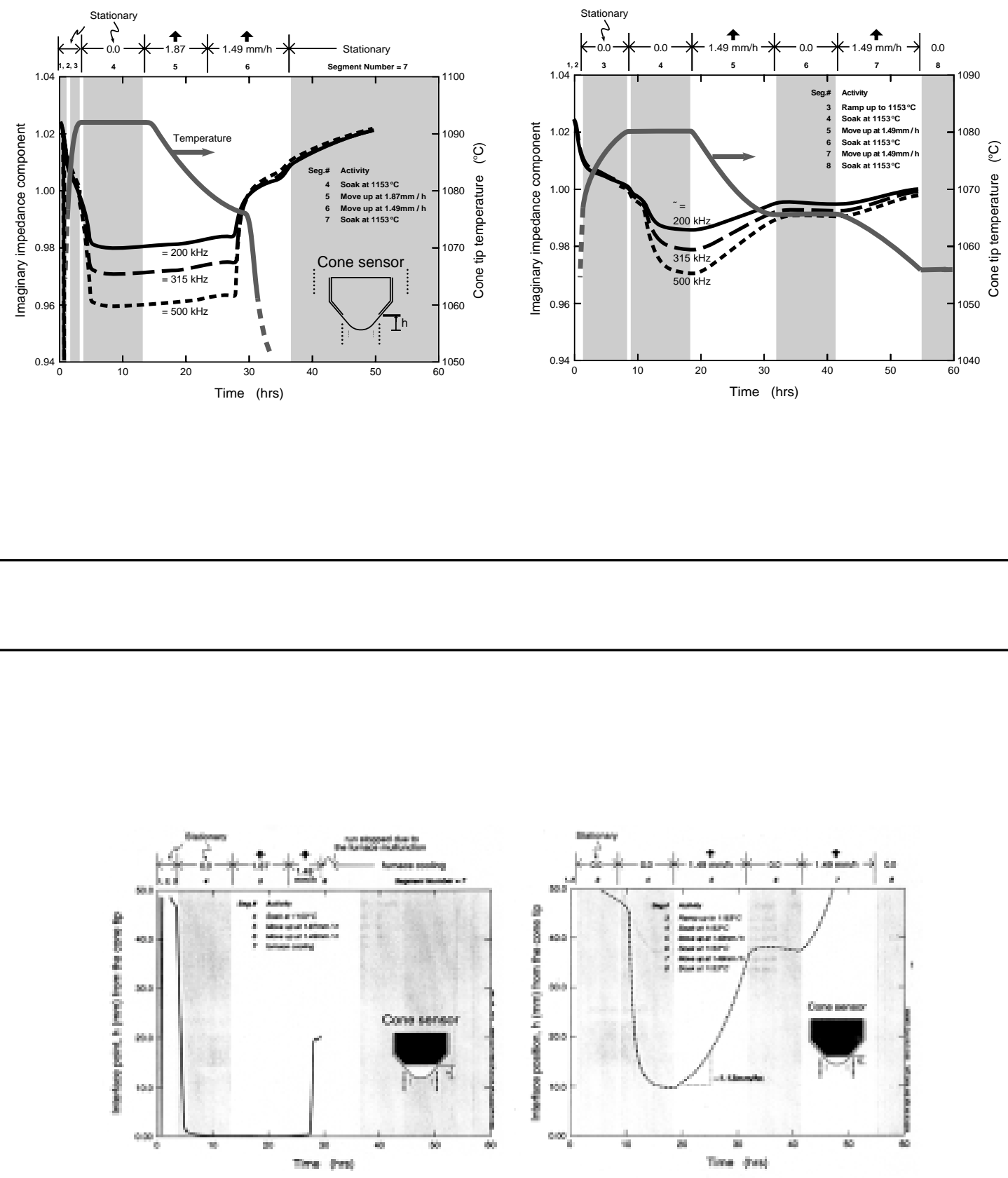
I M A G I N G A N D D E T E C T I O N

Initial Nucleation/Growth (seg. \#6)

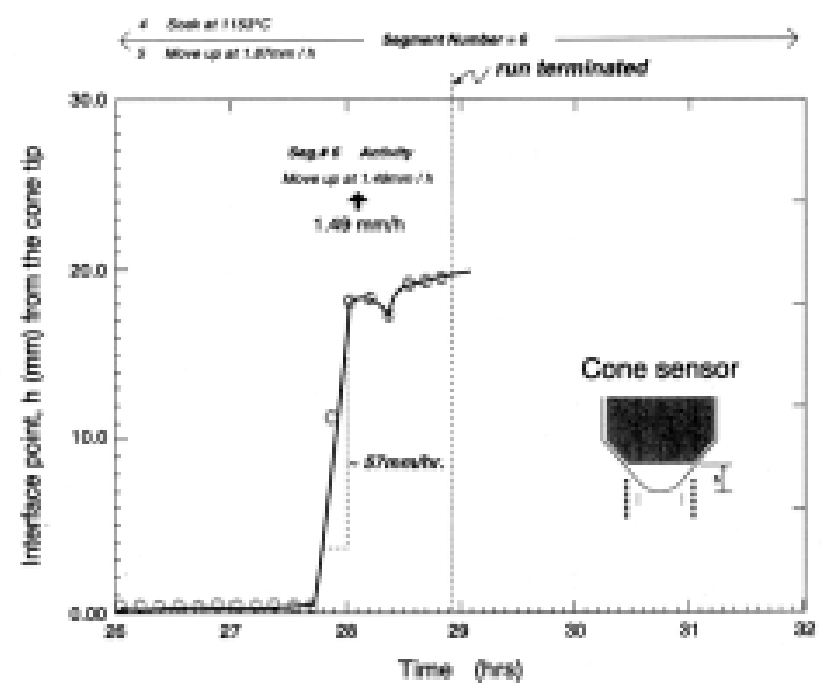

Photographs of the cone area showing the comparison of appearances between ingots grown

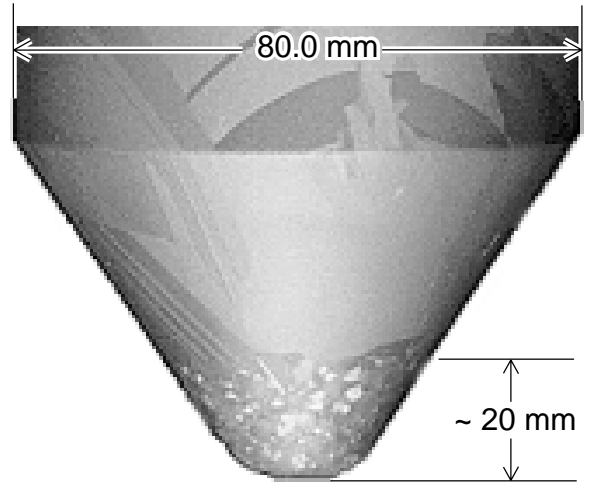

a) with completely melting state

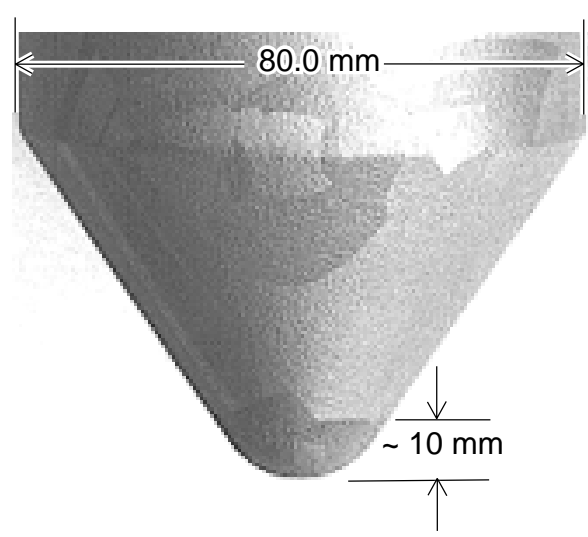

b) with incomplete melting 
I M A G I N G A N D D E T E C T I O N

\section{In-situ Study of Crystal Growth}

- Dual-coil encircling eddy current sensor system, non-contact, non-destructive

- Melt Stoichiometry

- Superheating and Undercooling

- Nucleation/Growth

- Solidification Velocity

- Interface Shape

- Temperature Gradients

- Cooling Rate etc.

- Strategies

- On-line sensing and feedback control

- Seeded solidification processing

Acknowledgements: UVa, JME, TI, and C. A. 


\section{Phase Retrieval in X-ray Diffraction}

\section{Abraham Szöke}

V Division

Lawrence Livermore National Laboratory

Livermore, CA 94551

\section{November 11, 1999.}

"... lack of information cannot be remedied by mathematical trickery"

Cornelius Lánczos

\section{X-ray Diffraction}

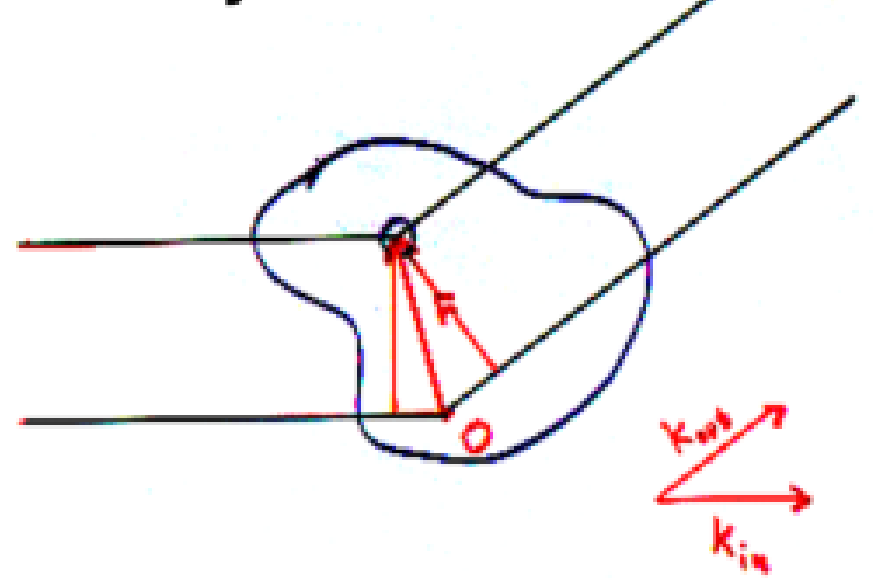

$I^{\prime}(\mathbf{h})-\int \rho(\mathbf{r}) \exp \left\{i\left(k_{\text {vut }}-k_{\text {in }}\right) \cdot \mathbf{r}\right\} d \mathbf{r}$ 


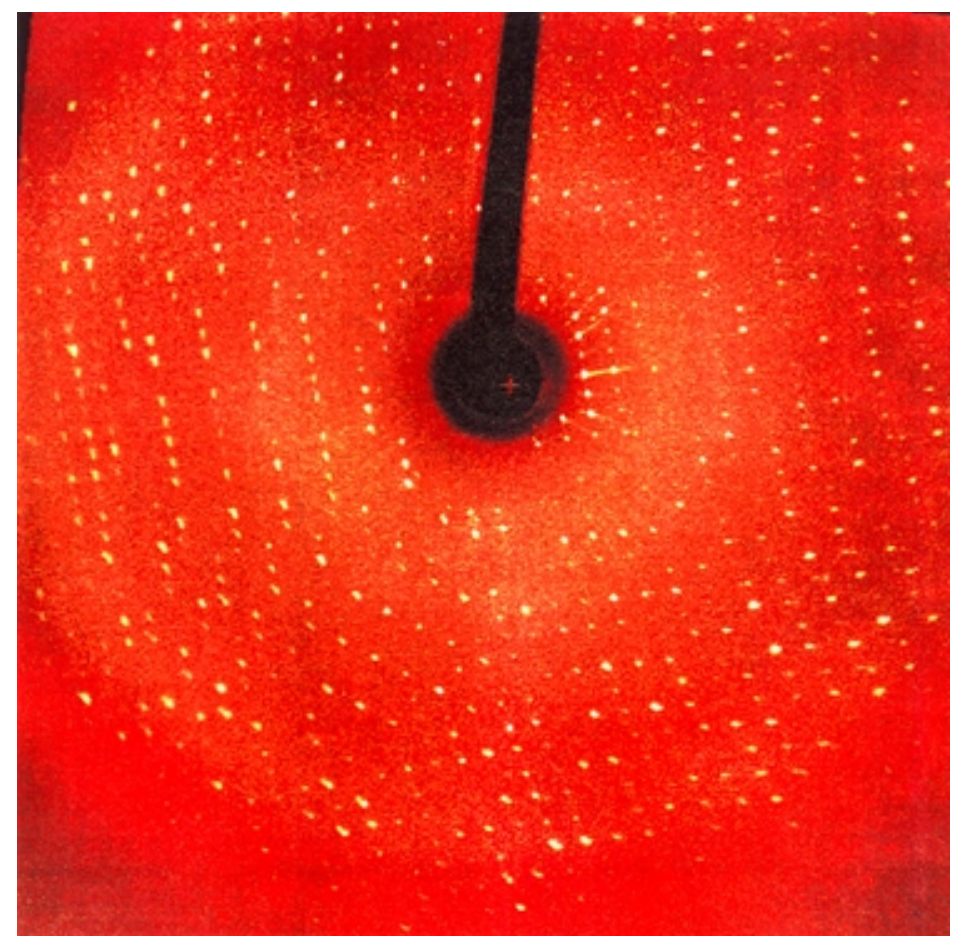

\section{The crystallographic "phase problem".}

The incident X-ray beam is usually coherent over may unit cells. Then, the diffraction pattern of a crystal is that of a unit cell,

$$
\mathrm{F}(\mathbf{h})=\int_{\text {unit cell }} \rho(\mathbf{r}) \exp (2 \pi \mathrm{ih} \cdot \mathbf{r}) \mathrm{dr} \text {. }
$$

It is sampled at integer values of the reciprocal lattice, $\mathbf{h}$.

Shannon's sampling theorem states that "a band limited function is completely determined by its sampled values, if the sampling is frequent enough."

Denote the Fourier transform of a function by $\mathrm{f}^{\wedge}(\mathrm{h})$. Band limit means that $\left|\mathrm{f}^{\wedge}(\mathrm{h})\right|=0$ if $|\mathrm{h}|>\Omega$. The theorem states that

$$
f(r)=\sum_{n} f(n \pi / \Omega) \frac{\sin (\Omega r-n \pi)}{(\Omega r-n \pi)}
$$

More is true: undersampling gives freedom; critical sampling converges slowly; oversampling gives rapid convergence. 


\section{The crystallographic "phase problem" (cont.)}

"... lack of information cannot be

remedied by mathematical trickery."

Cornelius Lánczos.

The Bragg condition results in integer values of $\mathbf{h}$. It is exactly critical sampling.

From the point of view of information theory, half the information is missing.

Measured amplitudes of the reflections with arbitrary phases give an equally "valid" electron density.

More information is needed.

\section{More, independent information:}

Experimental information:

MIR (Addition of the heavy atoms.)

MAD (Anomalous scattering by some atoms.)

NCS (Non crystallographic symmetry).

More than one crystal form of the same molecule.

Prior knowledge:

Positivity of the electron density.

Atomicity.

Nearly uniform density in solvent regions.

Knowledge of part of the molecule:

(Called molecular replacement.)

Model building:

(Called refinement.) 


\section{Can we produce the entire diffraction pattern of a molecule from a crystal?}

Local reference holograms do that.

Holographic Microscopy with a Local Reference

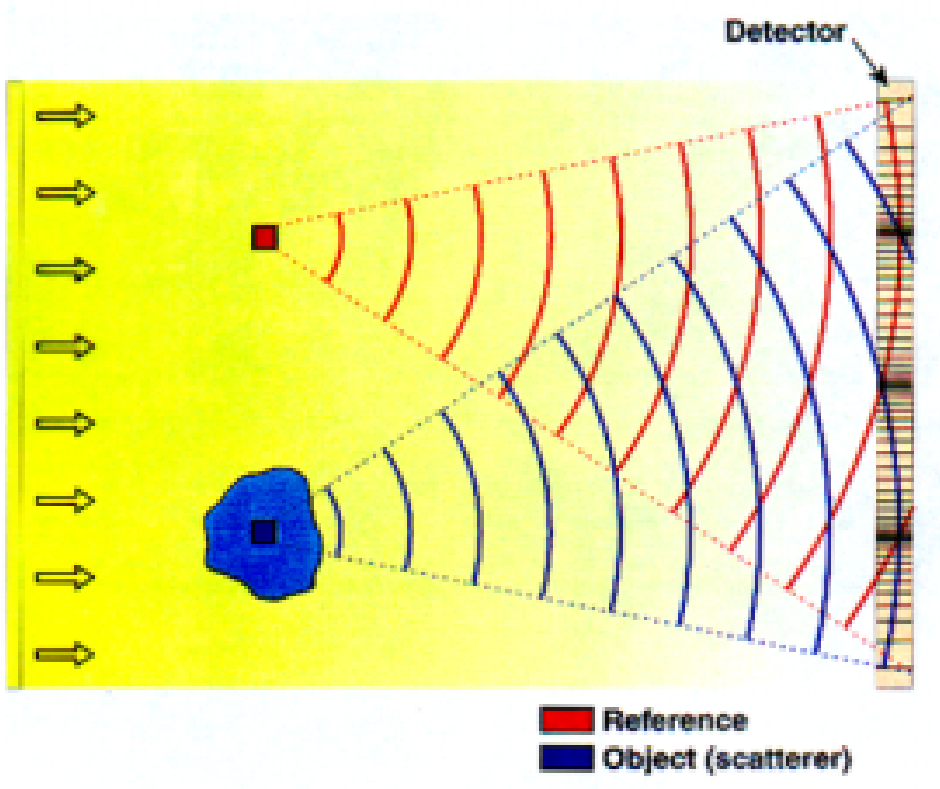



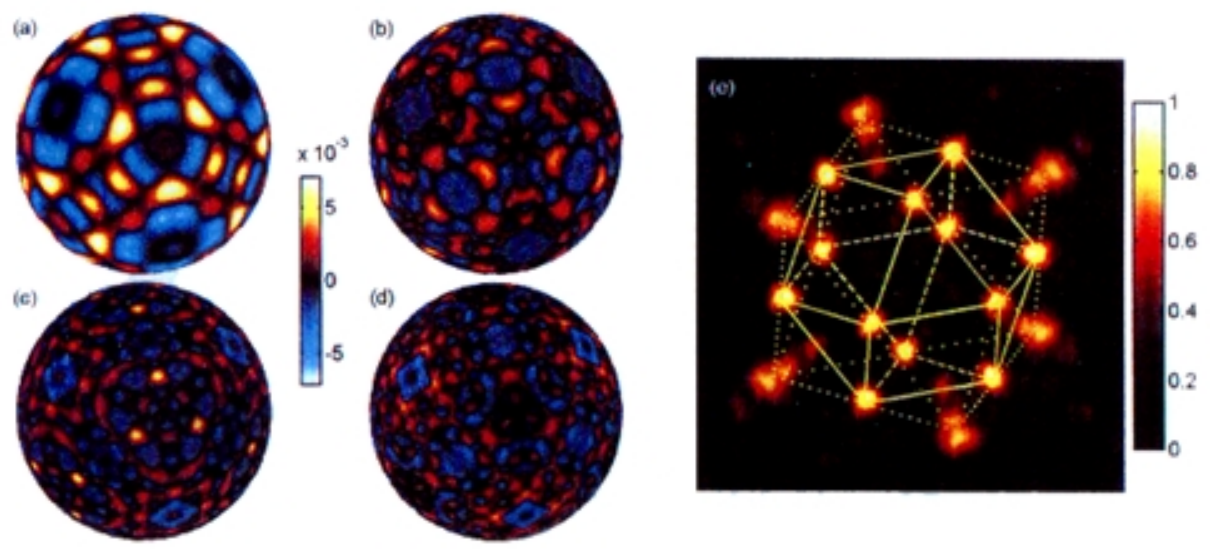

\section{Diffraction of a crystal with two kinds of molecules.}

We assume that the two kinds of molecules are similar and that they are thoroughly mixed.

We can define an average density of the two molecules, and a difference density.

- The average density is periodic: it gives Bragg peaks.

- The difference density produces a continuous diffraction pattern of the difference molecule.

\section{Time resolved chemistry in crystals:}

A reaction is initiated in a fraction of the molecules (e.g. by a short pulse laser.) A monochromatic X-ray pulse irradiates the crystal with a time delay. The diffraction pattern is recorded. 


\section{M A G I N G A N D D E T E C T I O N}

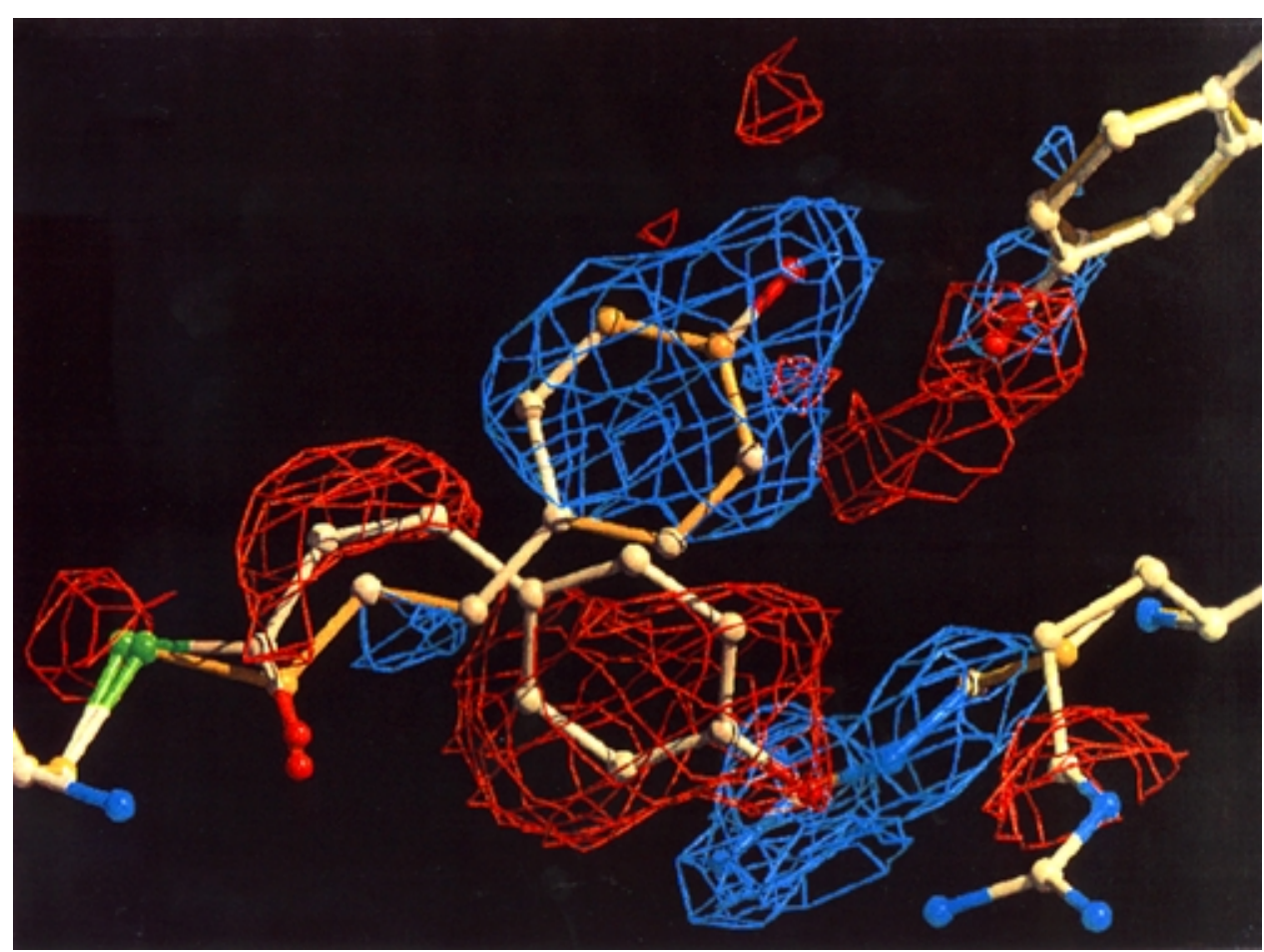

\section{Summary.}

There is a close relation between X-ray crystallography and holography. (Gabor credited Bragg with sparking his ideas.)

The diffraction pattern of a crystal is that of a single unit cell, sampled at the reciprocal lattice points. There is not enough information in the diffraction pattern to reconstruct the electron density.

A continuous diffraction pattern of a molecule can be sampled finely enough to reconstruct it. (Except for the enantiomorph, the Babinet opposite and the position of the origin. This ambiguity is analogous to the dual image problem of holography.)

I have shown several ways to produce a continuous diffraction pattern of a molecule using a crystal:

a local reference hologram,

a disordered overlayer,

a short coherence X-ray beam and

a crystal lattice randomly occupied by two different molecules. 


\section{Signal/Imaging}

Processing

for NDE 


\title{
Nondestructive Characterization Center Overview
}

\author{
Harry E. Martz, Jr. \\ Lawrence Livermore National Laboratory
}

The Nondestructive Characterization Center (NCC) supports initiatives that three to five years into the future will advance research, development, and cost effective application of nondestructive characterization science that enable successful LLNL and DOE programs. NCC plays a strategic and vital role researching and developing technologies to allow the Engineering Divisions to collaborate with and support LLNL and DOE programs. NCC pioneers nondestructive measurement science, develop and apply nondestructive measurement technology, and help transfer these advancements to LLNL and DOE programs, U.S. industry, universities, and other government agencies.

The NCC strategic mission objectives will advance core competencies and technologies in the following areas: (1) Quantitative NDE; (2) Fast Nanoscale Imaging; (3) Embedded in Design; (4) Multispectral; and (5) Portability. The NCC FY00 mission objectives advance core competencies and technologies vital to the following programs. Extraordinary laser systems, this includes advanced diagnostics, e.g., for fusion targets and automated in situ optics damage inspection. Weapon system performance, this includes 3D measurements over $15 \mathrm{~cm}$ to better than $25 \mu \mathrm{m}$, advanced diagnostics, e.g., Advanced Radiography Campaign and ceramic properties. Science-based stockpile stewardship, specifically durability and life prediction. Nonproliferation, arms control and international security related to reducing the threat from weapons of mass destruction. 

S I G N A L / I M A G E
$P$
R O
C E S S I N G
F O R
N D E

\section{Overview of the \\ Nondestructive Characterization Center}

prepared by

Harry E. Martz, Jr.

Nondestructive Characterization Center

Lawrence Livermore National Laboratory
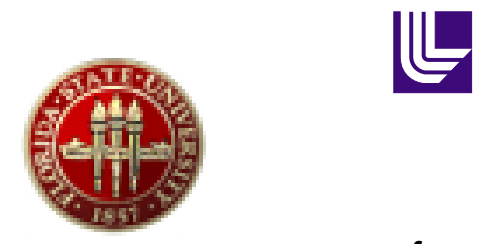

for

CASIS

1999 Signal and Imaging Sciences Workshop

Livermore, CA

November 12, 1999

The NCC's role is to R\&D the core technologies

and competencies to fulfill LLNL's mission

- Vision

- To be the World's leader in the research, development, and cost-effective application of nondestructive measurement science that enable successful LLNL and DOE programs, and a national science and technology resource for U.S. industry, universities, and other government agencies

- Mission

- Responsible for the vitality and growth of the NDC core technologies

- Focuses and guides investments in enabling and supporting capabilities

- Play a strategic and vital role researching and developing technologies to allow the Engineering Divisions to collaborate with and support LLNL and DOE programs

- Pioneer nondestructive measurement science, research, develop and apply nondestructive measurement technology

- Help Divisions transfer these advancements to LLNL and DOE programs, U.S. industry, universities, and other government agencies 


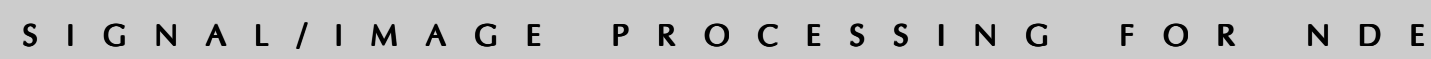

NDC system configurations are complex

integrations of hardware and software

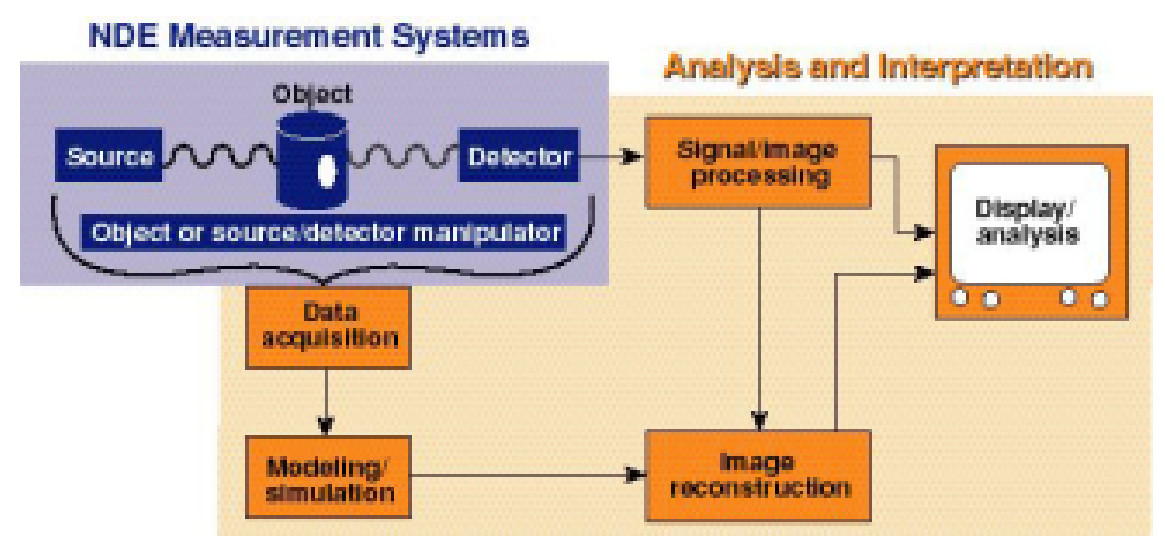

Multidisciplinary teams are essential in NDC R\&D, system integration and data interpretation to solve challenging programmatic problems

\section{The NCC strategic mission objectives will advance core competencies and technologies in five areas}

- Quantitative NDE-quantitative multi-dimensional nondestructive measurements to determine physical properties: geometry, density, elemental \& molecular composition, thermal, structural, mechanical, stress state, radioactive assay, etc., in all three dimensions and as a function of time for the materials/objects we characterize.

- Fast Nanoscale Imaging - provide multiple programs with quantitative nanoscale multidimensional (>3D) imaging.

- Embedded in Design-unite currently very diverse and somewhat non-coupled technologies such as design and modeling, materials development, characterization and testing, process monitoring and control, reverse engineering, and reuse and waste management into a global package of integration and iteration.

- Multispectral-expand the spectral range of NDE imaging technologies and to increase the synergism between different methodologies.

- Portability-The objective of portability is to reduce current and new NDE systems to a size that is on the order of a small suitcase. 

S I G N A L / I M A G E
$\begin{array}{llllllllll}\text { P } & R & O & C & E & S & S & \text { I } & N & G\end{array}$
F O R
N D E

\section{Improved Prosthetic Implant Designs Using Computed Tomography to FEA Methods}

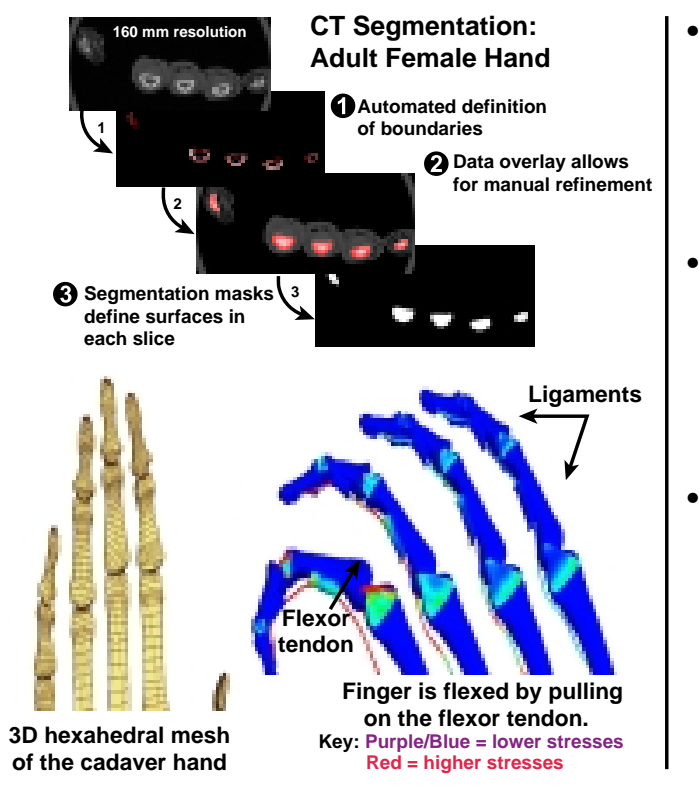

- Objectives

- New approach to evaluate and improve prosthetic joints before they are manufactured or surgically implanted

- Conversion of CT data to FEW models

- Challenges

- High definition 3D data of hard and soft human tissue

- Automated segmentation w/o artifacts

- CT and MRI data registration and fusion

- Status

- Cadaver hands and knees have been CT scanned and reconstructed

- The CT data have been segmented, surface rendered and meshed

- FEA results have been used to predict failure and to improve implant designs

\section{Compelling NCC problems}

- Measurement Systems

- See inside objects beyond what has been seen before

- Improve spatial resolution down to $\mathrm{nm}$ scale

- Increased penetration/contrast to see inside materials not accessible before

- Temporal (fast) volumetric imaging

- Analysis and Interpretation

- Multi-dimensional ( $>3 D$ ) signal and image processing

- Speed, accuracy and noise reduction in processing

- Data interpretation-We see more and more things; what does this mean to the customer

- Fusion of different measurement systems data

Measurement systems, and analysis and interpretation must be developed jointly to get optimal synergy 


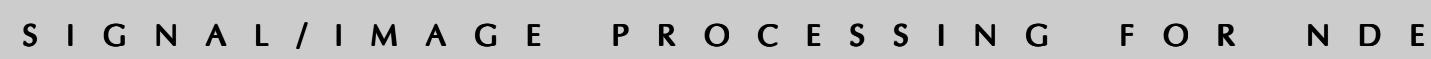

\section{Automated multi-dimensional data acquisition, processing and interpretation is the world of the future}

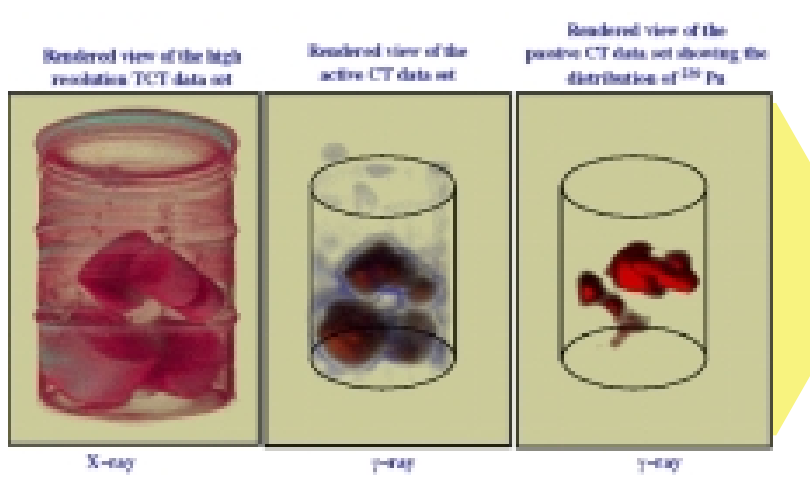

Data Reduction:

3 grams

weapons grade

plutonium

Decision:

Transuranic (not low-level) waste disposal required

Typically multi-dimensional data are required to produce a single number to make a crucial decision

"GigaBytes to a Bit"

\section{Capabilities that would fundamentally alter the way our customer does business}

- Weapons surveillance

- Weapon system performance, this includes three-dimensional measurements over a large size with high spatial resolution, and advanced diagnostics, e.g., Advanced Radiography Campaign, and materials properties measurements

- Science-based stockpile stewardship, specifically durability and life prediction

- Precision diagnostics and devices

- Extraordinary laser systems, this includes advanced diagnostics, e.g., for fusion targets and automated in situ optics damage inspection

- Arms control, nonproliferation, and international security related to reducing the threat from weapons of mass destruction

- Portable instruments // Imaging // Remote Sensing 
S I G N A L / I M A G E P R O C E S S I N G F O R N D E

\section{Details of NCC's future driving applications and collaboration with other Centers}

- $\quad$ Team with PSEC \& $\mathrm{C} \mu \mathrm{T}$ in NIF target fabrication/characterization

- Characterization/metrology

- Diagnostics

- Team with CCE on NDE data to computational models \& analysis

- Simplify and automate the process to convert the NDE data to FEA meshes and data analysis

- Adapt models to incorporate the new information provide by NDE

- Research and develop life prediction

- Nano-scale characterization facility for materials characterization to predict life cycle in situ

- Mechanical testing via micro- to nano-tensile testing, indentation, characterization in situ, etc.

- Cycle back into design and fabrication

In order for this to be successful we also need to work very closely with Engineering's Divisions and LLNL Programs

\section{NCC's LDRD Exploratory Research portfolio}

- Quantitative Tomography Simulations and Reconstruction Algorithms-Martz

- Straight-ray (CTSIM/HADES) and Monte Carlo (COG/MCNP) simulations

- Validation of simulation models with experimental DR and CT data

- Better object recovery by incorporation of physics in forward model

- $\quad$ Speckle Reduction for LIDAR-Bowers

- Speckle compensation through nonlinear-optical techniques

- Speckle modeling software with nonlinear-optical subroutines

- Improved performance of LIDAR systems for faster, more accurate, results

- High Resolution and Fast Imaging Photothermal Microscopy-Chinn

- Imaging of micron-sized defects for 106 improvement in speed

- Increased imaging sensitivity through optical lock-in hardware

- Nanometer resolution in scanning using optical fiber tips 
S I G N A L / I M A G E P R O C E S S I N G F O R N D E

\section{NCC s FY00 Technology Base portfolio}

\begin{tabular}{|l|l|l|}
\hline & PI & TITLE \\
\hline & & \\
\hline & Priority list of proposals & \\
\hline & & \\
\hline 1 & Landram & Flaw Detection Using Infrared Methods \\
\hline 2 & Blaedel $* *$ & The conceptual design of a facility for manufacturing meso-scale devices for NIF \\
\hline 3 & Chambers & Extended Resolution Diffraction Tomography \\
\hline 4 & Decker & Advanced x-ray sources \\
\hline 5 & Dolan & Near Nanoscale X-ray Computed Tomography with Low to Medium Energy X-rays \\
\hline 6 & Thomas & Ultrasonic Phased Arrays \\
\hline 7 & Roberson & True Three Dimensional Signal Processing Tool Development \\
\hline 8 & Kallman & Rapid, High Resolution Ultrasound Tomography \\
\hline 9 & Jackson & RECON-Code Maintenance \\
\hline
\end{tabular}

Target Fab./Char. Related ${ }^{\star \star}$ To be presented in PSEC or MMED presentation

\section{The NCC s projects supports three LLNL programs directly and others indirectly}

\begin{tabular}{|l|c|c|c|c|c|c|}
\hline \multicolumn{1}{|c|}{ Focus areas } & D\&NT & Lasers & NAI & Energy/Env & Bioscience & Other \\
\hline Quantitative NDE & $\begin{array}{c}\text { HR1-Martz } \\
\text { T1 Landram, T2-Blaedel; } \\
\text { T3-Chambers; } \\
\text { T6-Thomas; T7-Kallman; } \\
\text { T8 Jackson }\end{array}$ & $\begin{array}{c}\text { H T2-Blaedel; } \\
\text { T8 Jackson }\end{array}$ & M R2-Bowers & H T8 Jackson & H & H \\
\hline Fast Nanoscale Imaging & H T4-Decker; T5-Dolan & $\begin{array}{c}\text { H T3-Chinn } \\
\text { T4-Decker; T5-Dolan }\end{array}$ & M & M T5-Dolan & H & H \\
\hline Embedded in Design & $\mathbf{H}$ & H & M & H & M & H \\
\hline Multispectral & $\mathbf{M}$ & H & H & H & H & M \\
\hline Portability & $\mathbf{M}$ & M & H & H & M & M \\
\hline
\end{tabular}

\section{Objectives}

Quantitative NDE The objective of quantitative multi-dimensional NDE is to obtain measurements to evaluate physical properties: geometry, density, elemental \& molecular composition, thermal, structural, mechanical, radioactive assay, etc., in all three dimensions and as a function of time for the materials/objects we characterize. We set a goal of $0.1 \%$ accuracy for each of these properties at videoframe rates. This includes integration of inspection, evaluation and characterization methods to prodict material response and life times, and when necessary, reduce data to a quantitative decision.

Fast Nanoscale Imaging The fast Nanoscale imaging objective is to provide multiple programs with quantitative multidimensional $(>3 D)$ imaging down to nanoscale lengths. Most techniques that provide such a capability to date are surface and near surface methods. Our goal is to do this on a specimen in bulk/3D, i.e., beyond nearsurface. We want to develop NDE techniques that can obtain spatial resolution of $100 \mathrm{~nm}$ (resolve two objects separated by $100 \mathrm{~nm}$ ) over 1-mm field-of-view at video frame rates. We need to determine if superresolution is possible and useful to obtain spatial resolutions to better that the diffraction limit of the NDE imaging systems.

${ }^{\dagger} \mathrm{D} \& \mathrm{NT}$ is Defense \& Nuclear Technologies, NAl is Nonproliferation, Arms Control,\& International Security; Others refers to work outside of DOE, eg., DOT, DOD and private sector; Key: $\mathrm{H}$ is high; $\mathrm{M}$ is medium; and $\mathrm{L}$ is low impact to the program; $\mathrm{R}$ is LDRD and $\mathrm{T}$ is Tech. Base. $10 / 28 / 99$ 
S I G N A L / I M A G E P R O C E S S I N G F O R N D E

\section{Ultrasonic Activities at Lawrence Livermore National Laboratory}

Graham Thomas

Ultrasonic energy can be generated and detected with lasers

\section{Pulsed laser sourde}

\section{Interferometer}

Transient recorde凨形
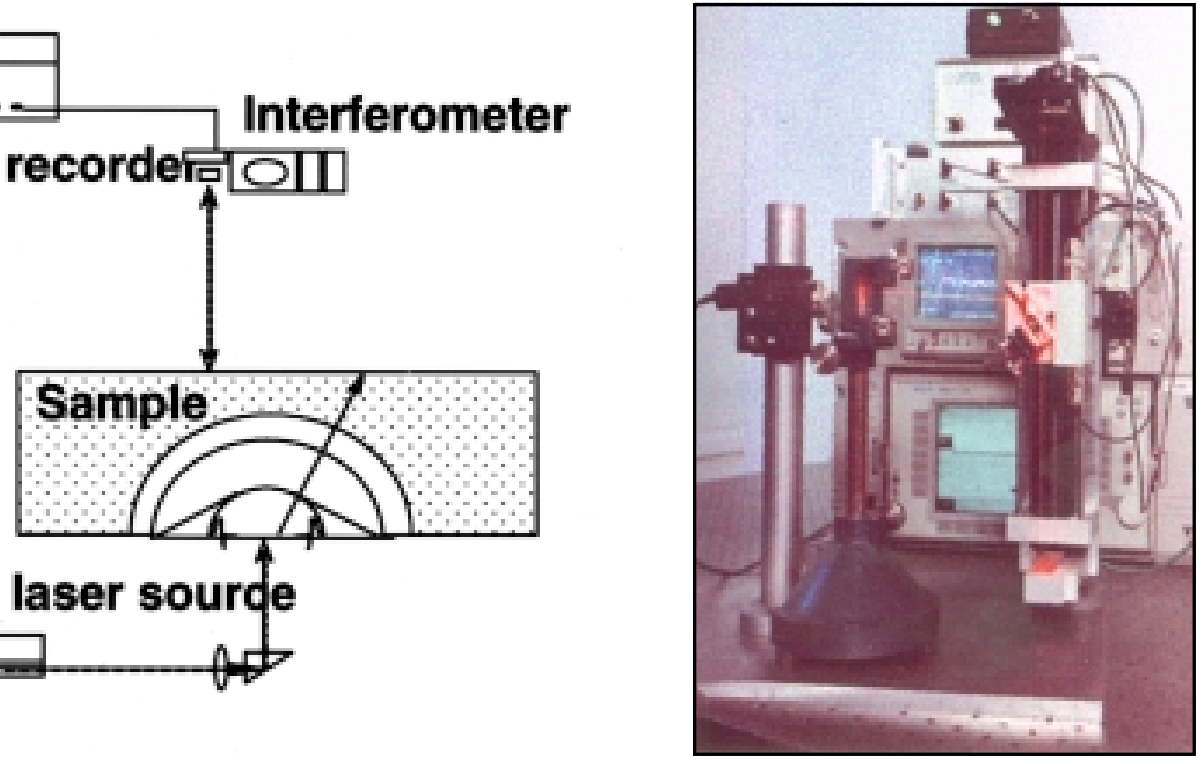
S I G N A L / I M A G E P R O C E S S I N G F O R N D E

Laser interferometer will sense the change in the acoustic signal generated by cutting laser
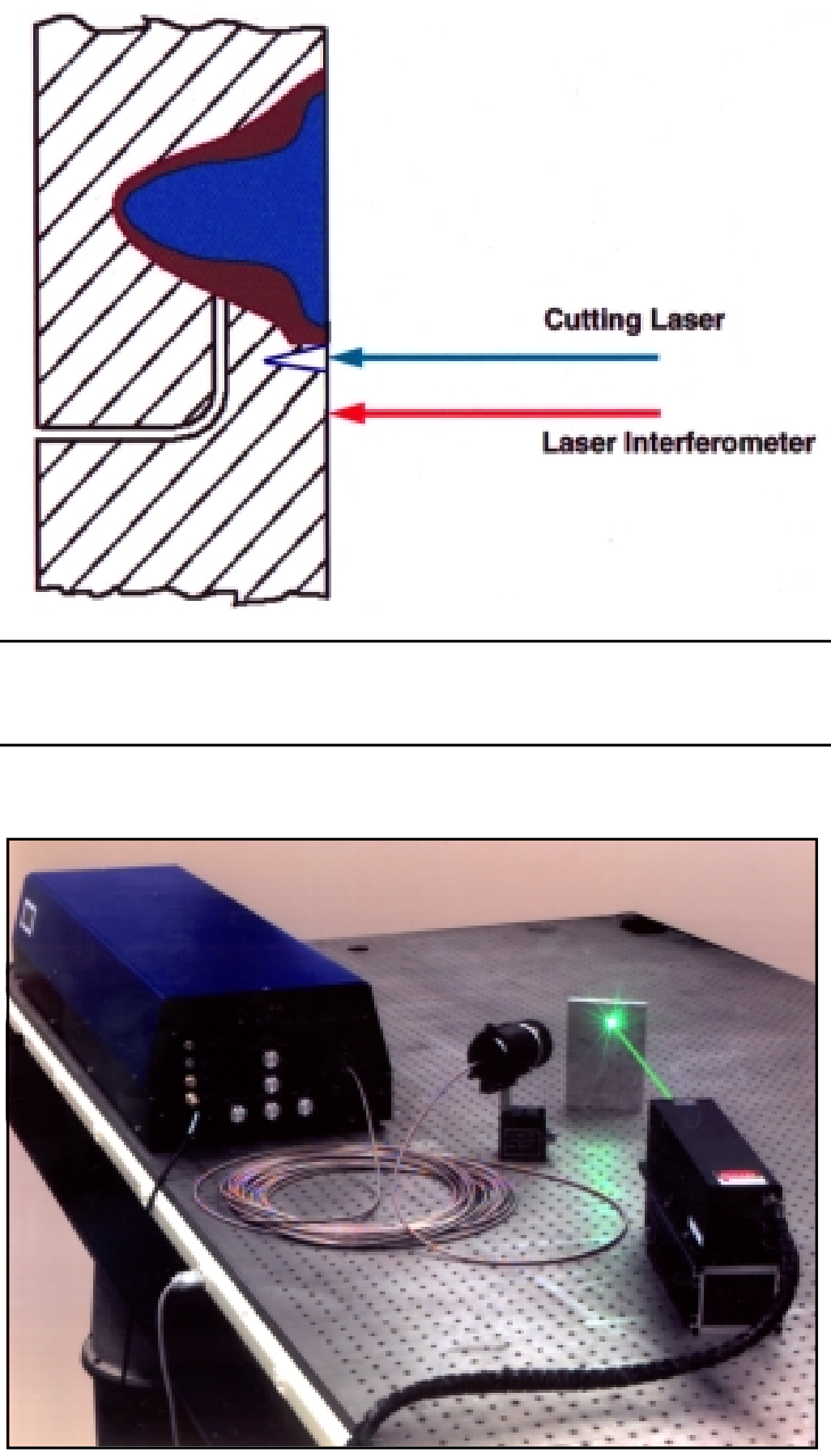
S I G N A L / I M A G E P R O C E S S I N G F O R N D E

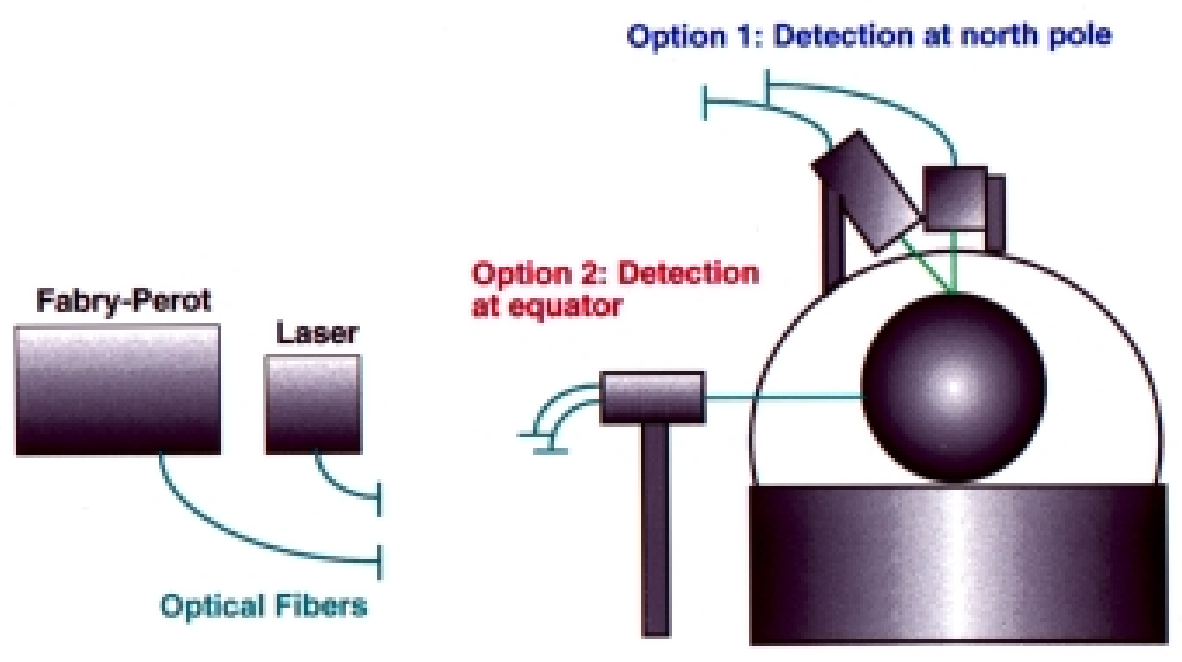

An ultrasonic signal decreases with cutting laser penetration depth
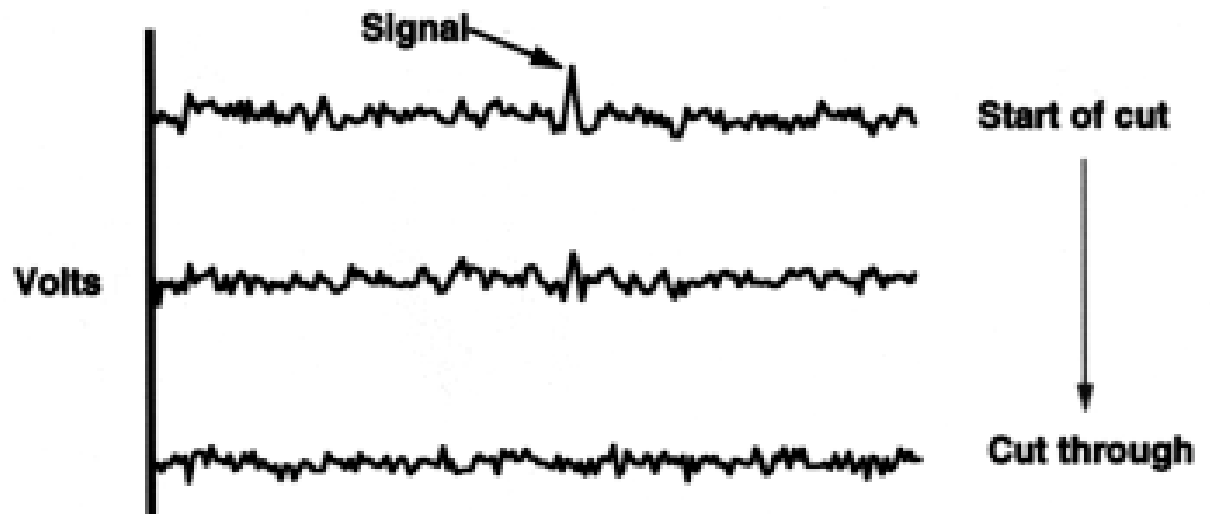

Time (usec.)

Data from interferometer pointed at the north pole 
S I G N A L / I M A G E P R O C E S S I N G F O R N D E

We derived an empirical equation relating failure strength to ultrasonic data

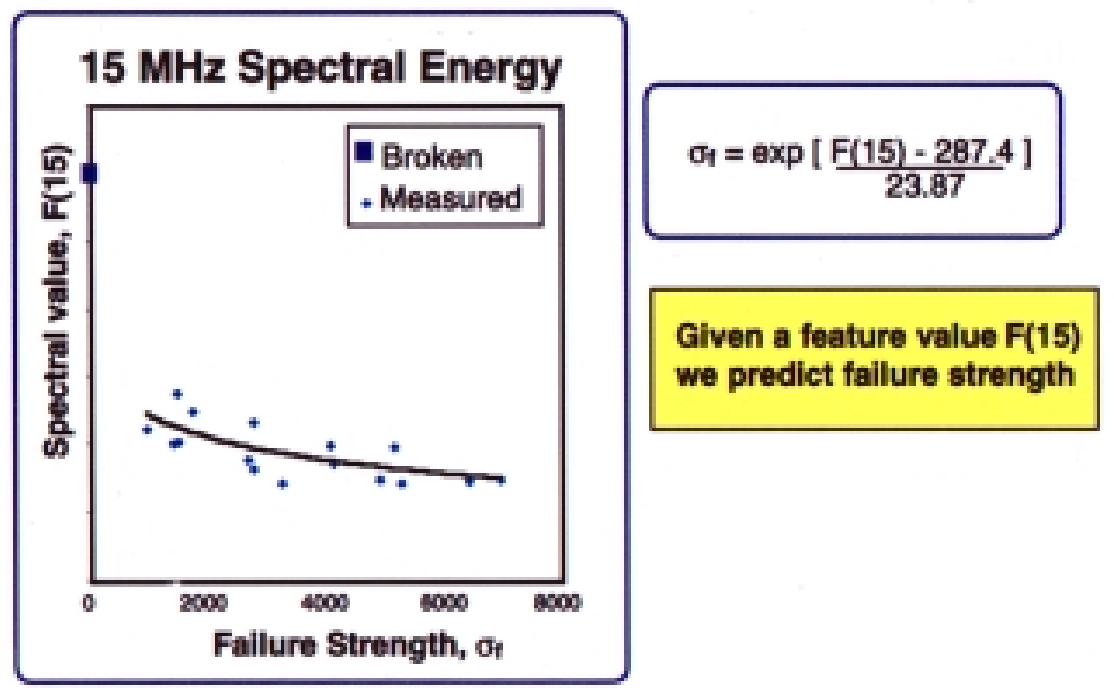

Ultrasonic features correlate with microstructure and failure strength
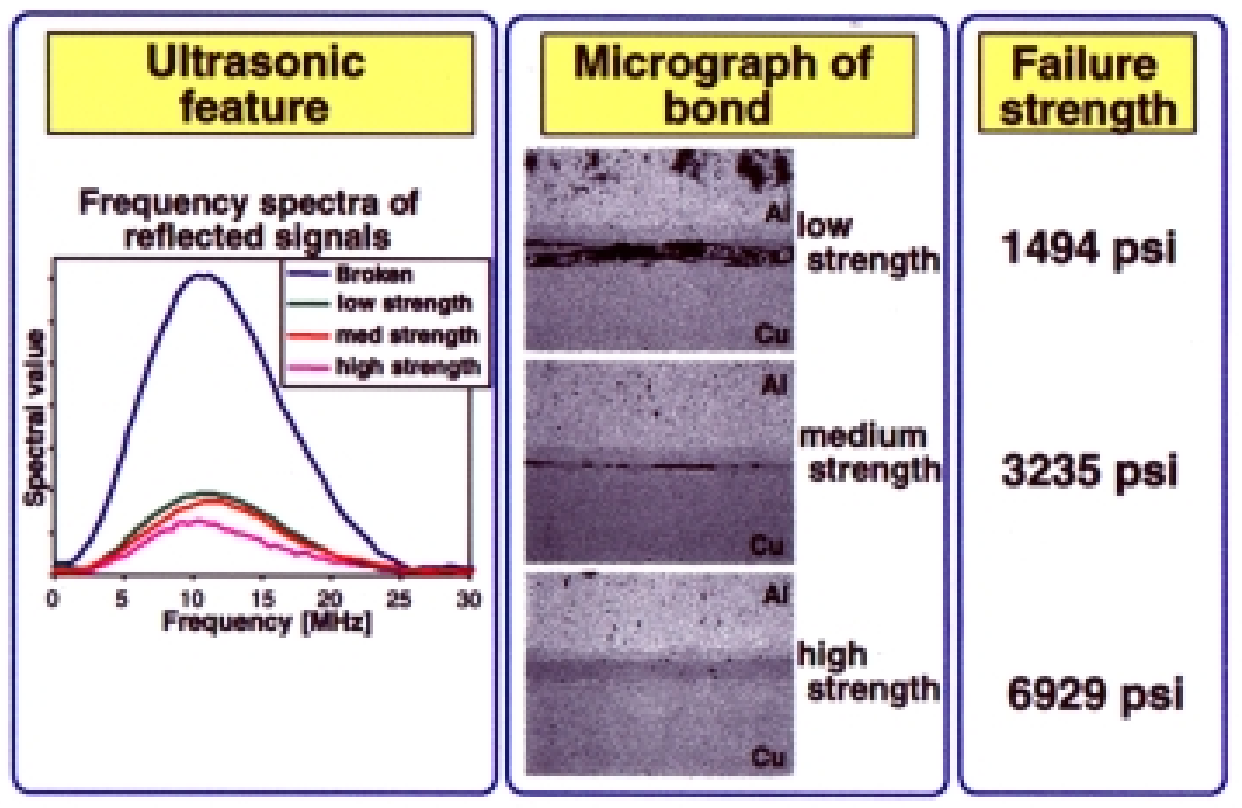

S I G N A L / I M A G E
P R O C E S S I N G
F O R
N D E

We fabricated bond samples to characterize quality

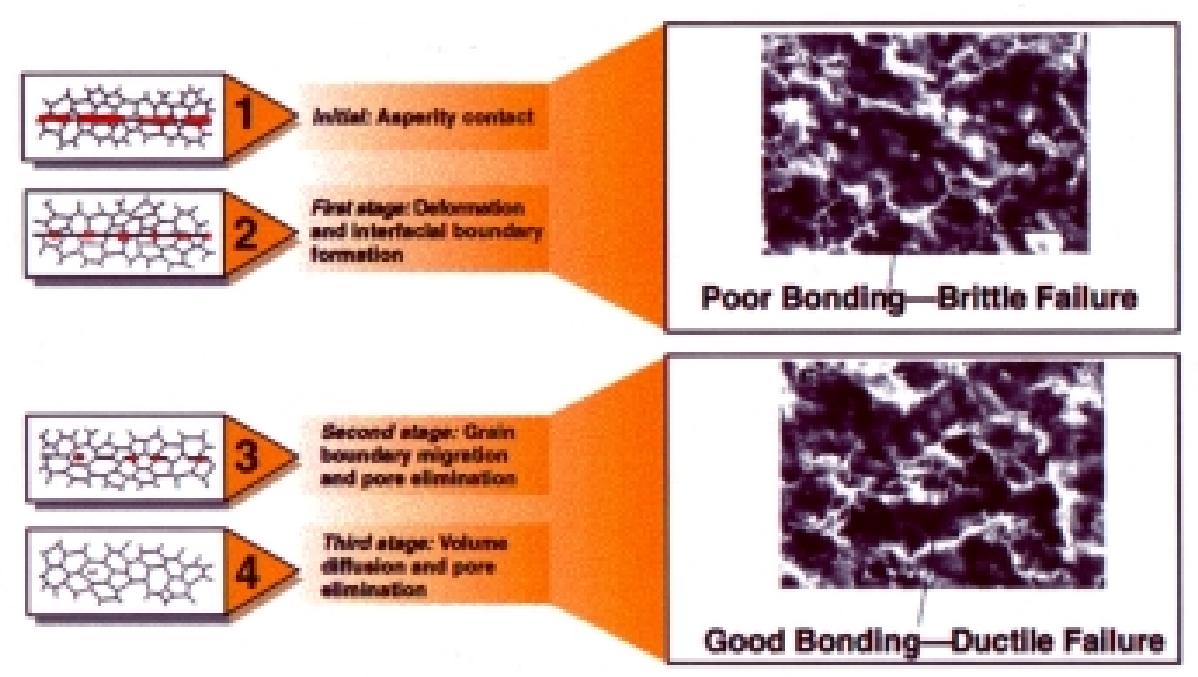

Tensile specimens were tested to give us the bond quality

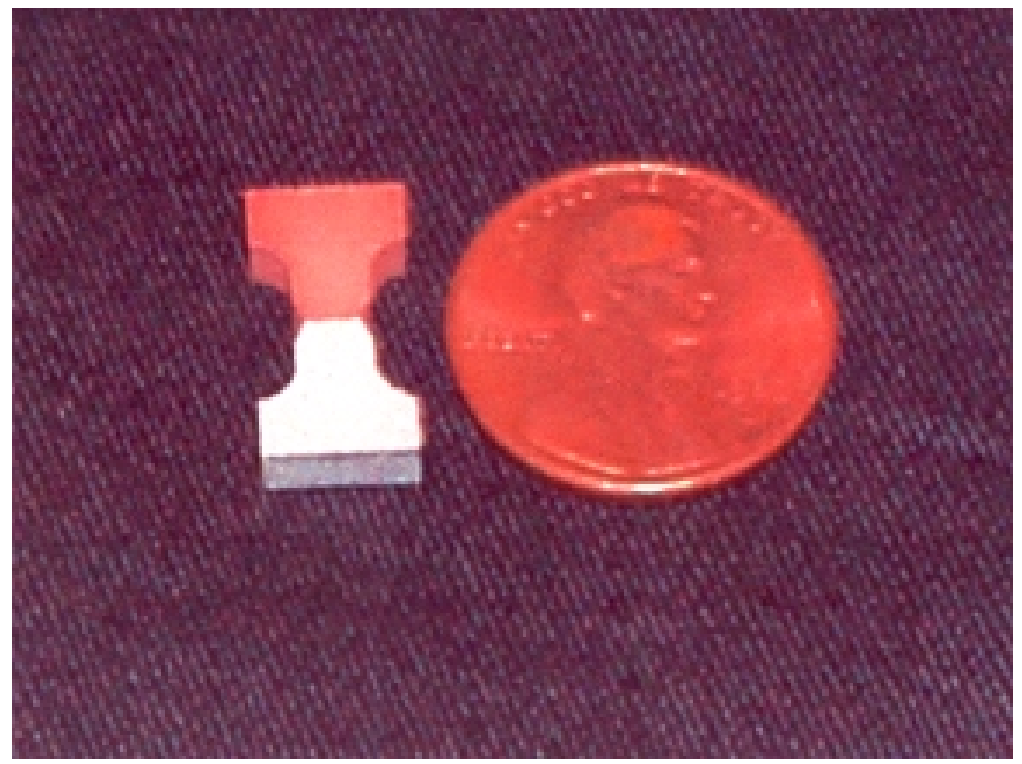


S I G N A L / I M A G E P R O C E S S I N G F O R N D E

High resolution images currently display nonbonded regions

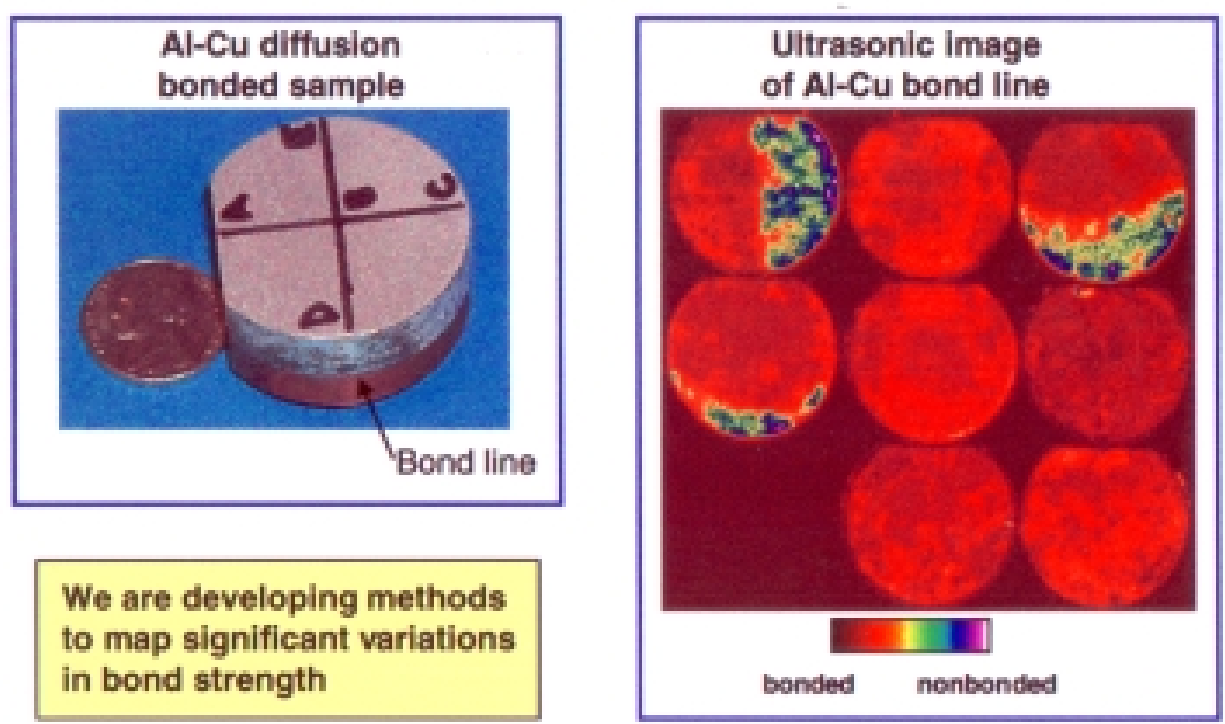

\section{Experimental protocol has produced a correlation} between bond strength and ultrasonic signals

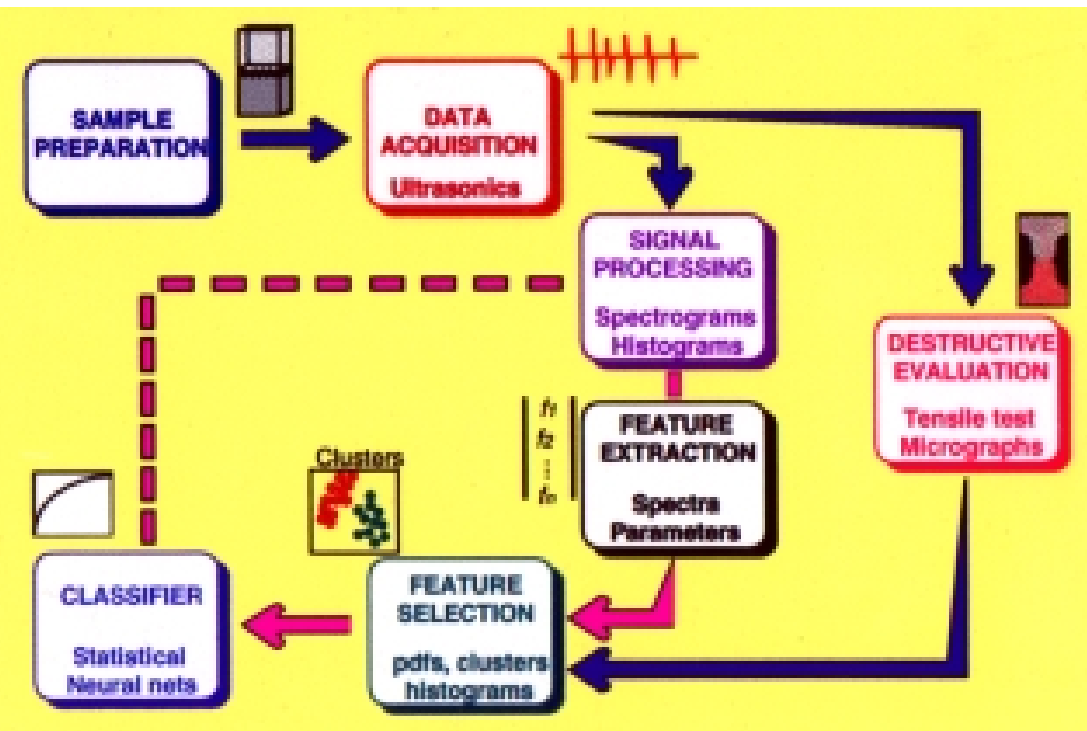



S I G N A L / I M A G E
$\begin{array}{lll}P & \mathbf{R} & \end{array}$
C E S S S I I N G
F O R
N D E

Ultrasonics displays defects in 3-D so that their size, location, and orientation can be determined

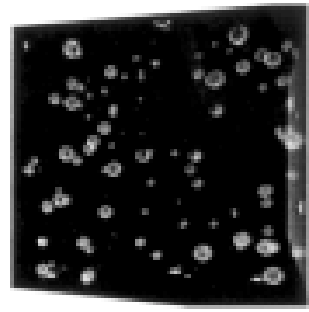

0 degree view

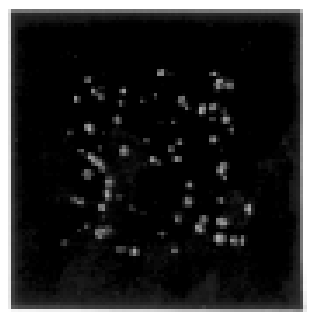

Rotated 180 degrees

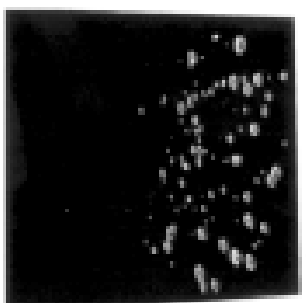

Rotated 90 degrees

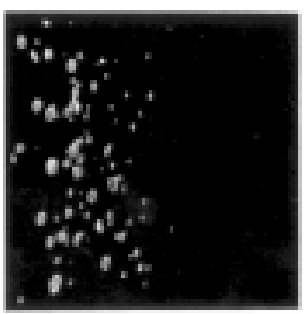

Potated 270 degrees

Combining $x$ and $y$ location with depth information provides 3-D images

Suspended air bubbles in epoxy

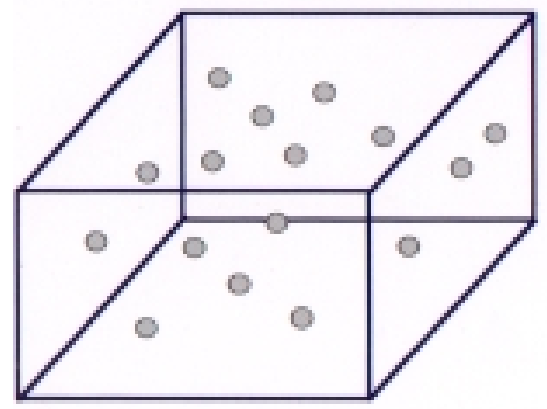

3-D UItrasonic imaging technique

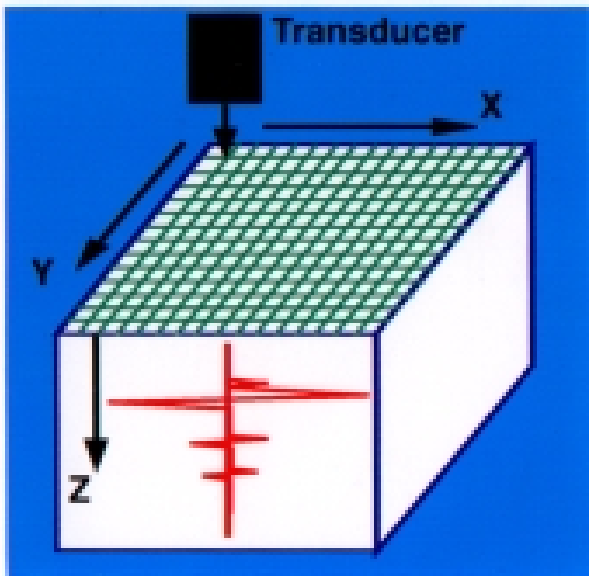


S I G N A L / I M A G E P R O C E S S I N G F O R N D E

Ultrasonic data can be presented in several formats

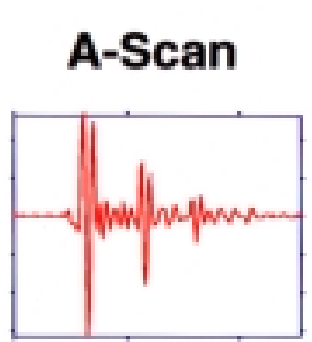

Oscilloscope time domain signal (Single location depth information)

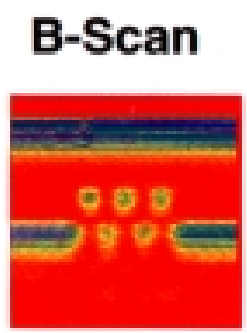

Cross sectional view (Depth information along a line)
C-Scan

\section{0}

000

000

$=$

Plan view of sample

( $X$ and $Y$ position information)

\section{Ultrasonic C-scans provide planar view} of reflectors at a prescribed depth

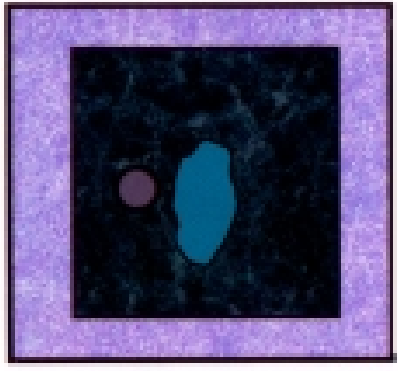

Transducer

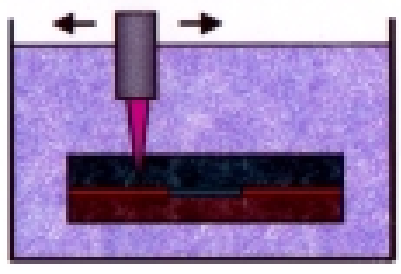

C-scan image

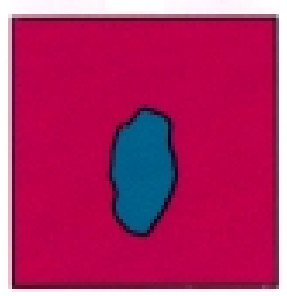

Front wall

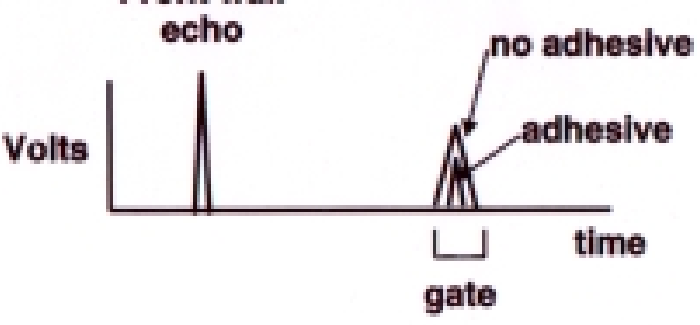



S I G N A L / I M A G E
P R O C E S S I N G
F O R
N D E

Ultrasonic nondestructive evaluation interrogates components with high frequency acoustic energy
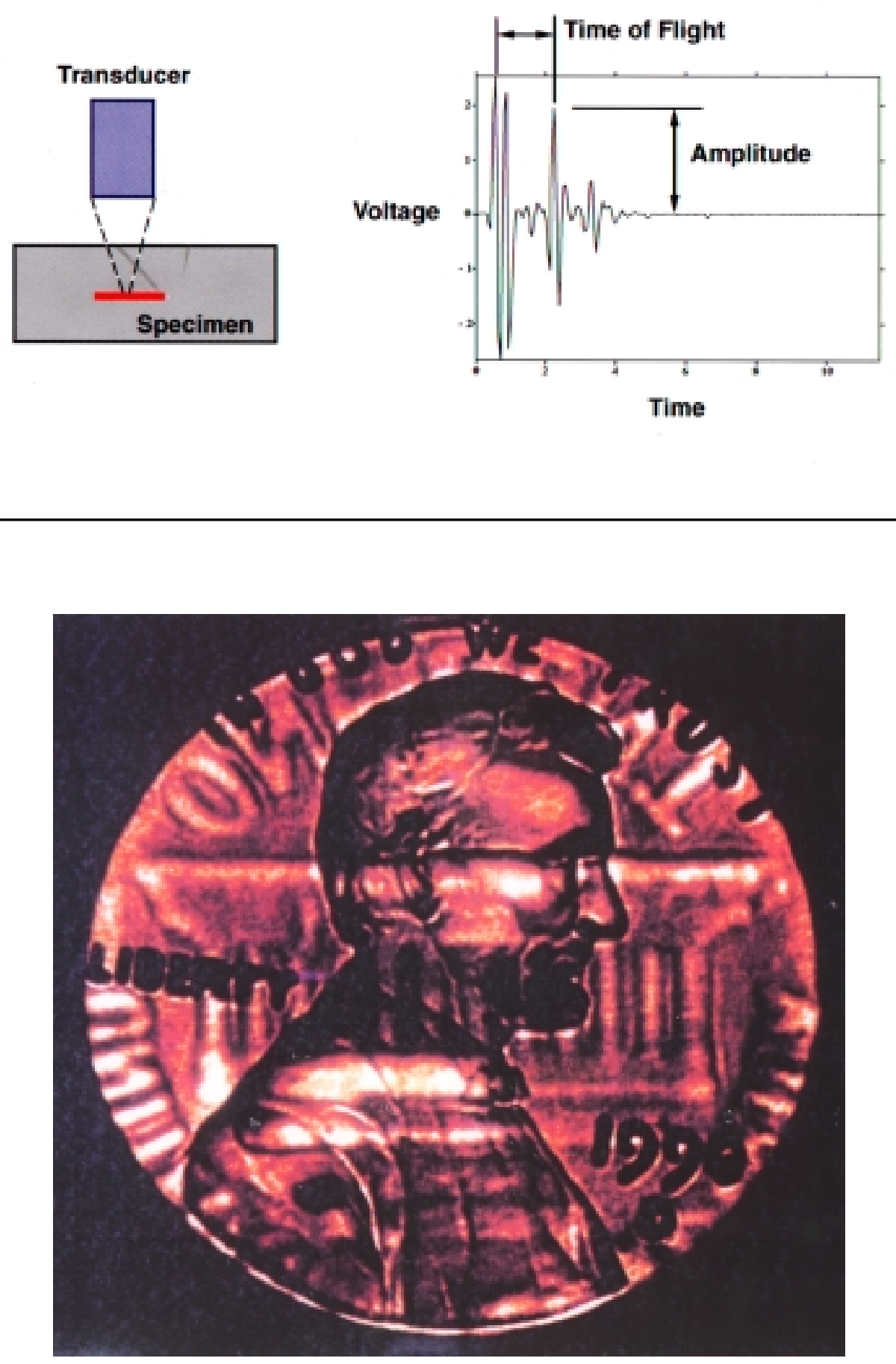
S I G N A L / I MAGE PROCE S S I NG F O R N D E

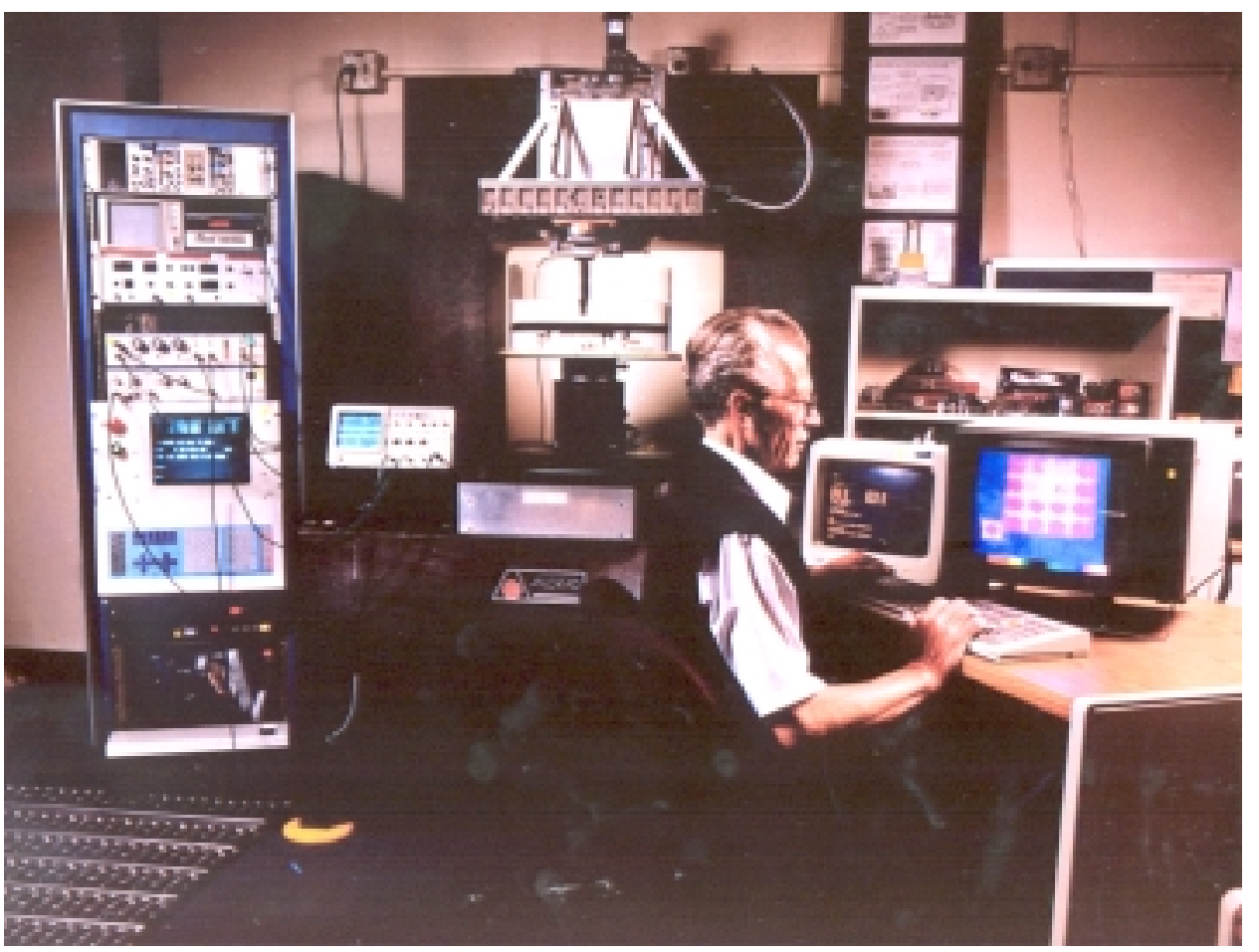


S I G N A L / I M A G E P R O C E S S I N G F O R N D E

\section{Signal Processing for Laser Ultrasonic NDE}

R. Huber, J. Candy

LAWRENCE LIVERMORE NATIONAL LABORATORY

ORGANIZATION

- INTRODUCTION: Laser Ultrasonics

- SIGNAL PROCESSING: Correlation Cancelling

- Summary

LAWRENCE LIVERMORE NATIONAL LABORATORY 
S I G N A L / I M A G E P R O C E S S I N G F O R N D E

\section{LASER ULTRASONICS}

LAWRENCE LIVERMORE NATIONAL LABORATORY

\section{Laser-based Ultrasound}

- Allows non-contact, remote testing.

- Allows testing in vessels, furnaces.

- Nondestructive.

- Linear broadband.

- Works with complicated geometries.

LAWRENCE LIVERMORE NATIONAL LABORATORY 
S I G N A L / I M A GE PROCESS I NG F O R N D E

\section{Laser-Based Ultrasound System}

- Pulsed Nd: YAG laser.

- Stabilized HeNe-based Michelson interferometer

- Computer-controlled motion stages.

LAWRENCE LIVERMORE NATIONAL LABORATORY

Displacements can accurately be measured using a laser interferometer similar to the simple setup here:

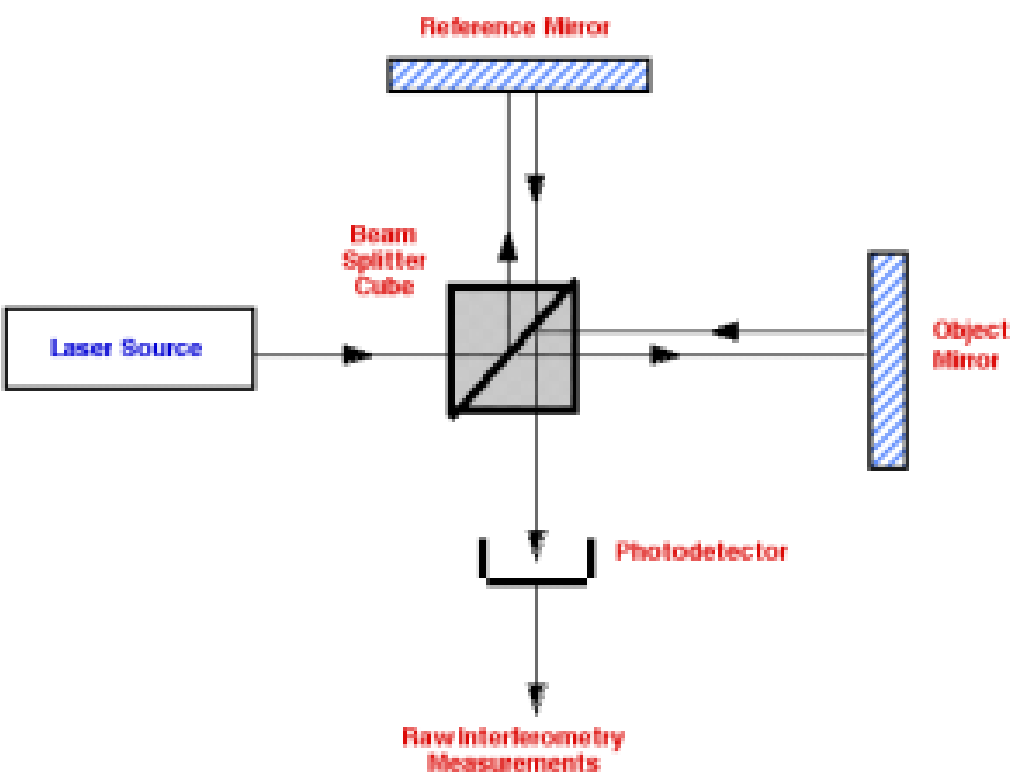


S I G N A L / I M A G E P R O C E S S I N G F O R N D E

Ultrasonic waves are generated through the thermoelastic expansion of the material created by laser heating

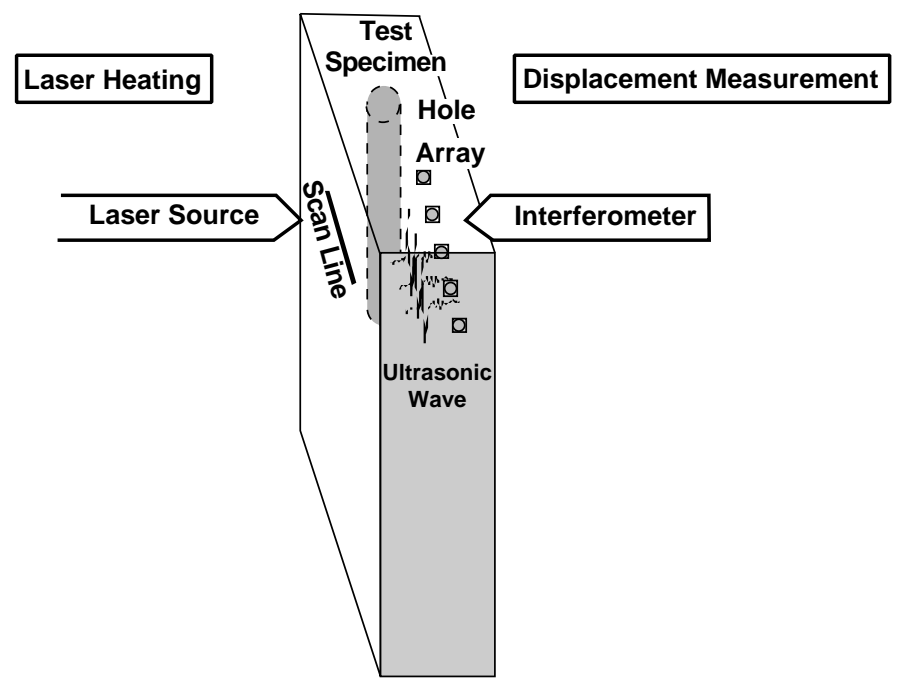

LAWRENCE LIVERMORE NATIONAL LABORATORY

A typical epicentral waveform has the following characteristics:

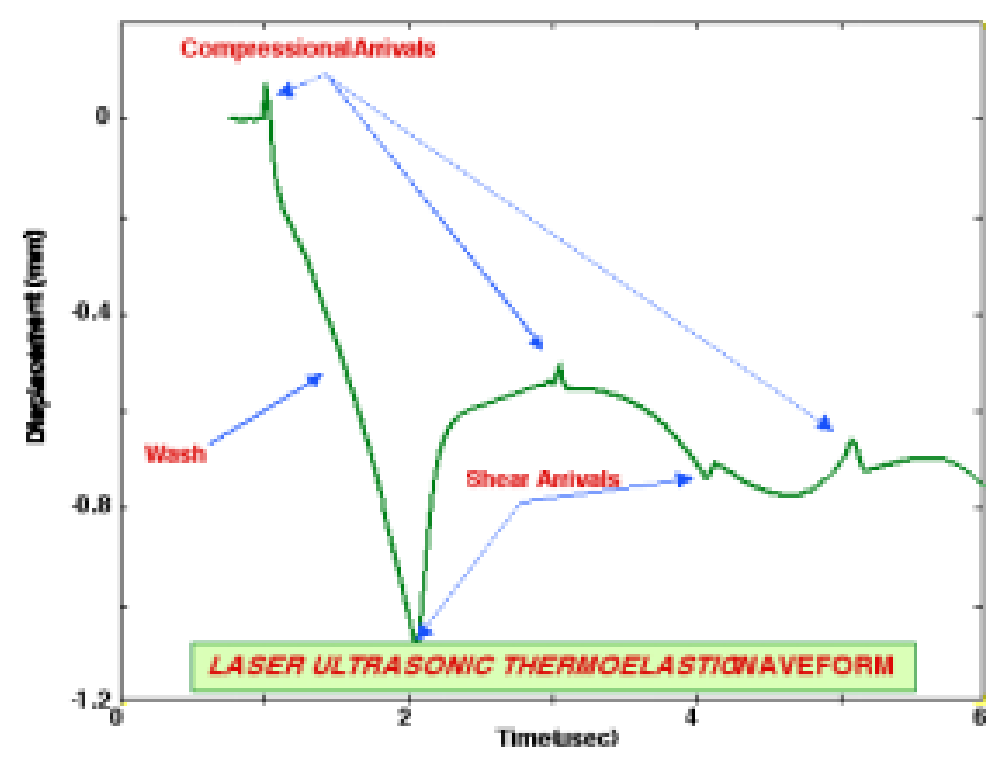


S I G N A L / I M A GE PROCESS I NG F O R N D E

Surface displacements can be measured by a sensitive laser interferometer to create a synthetimultichannel array

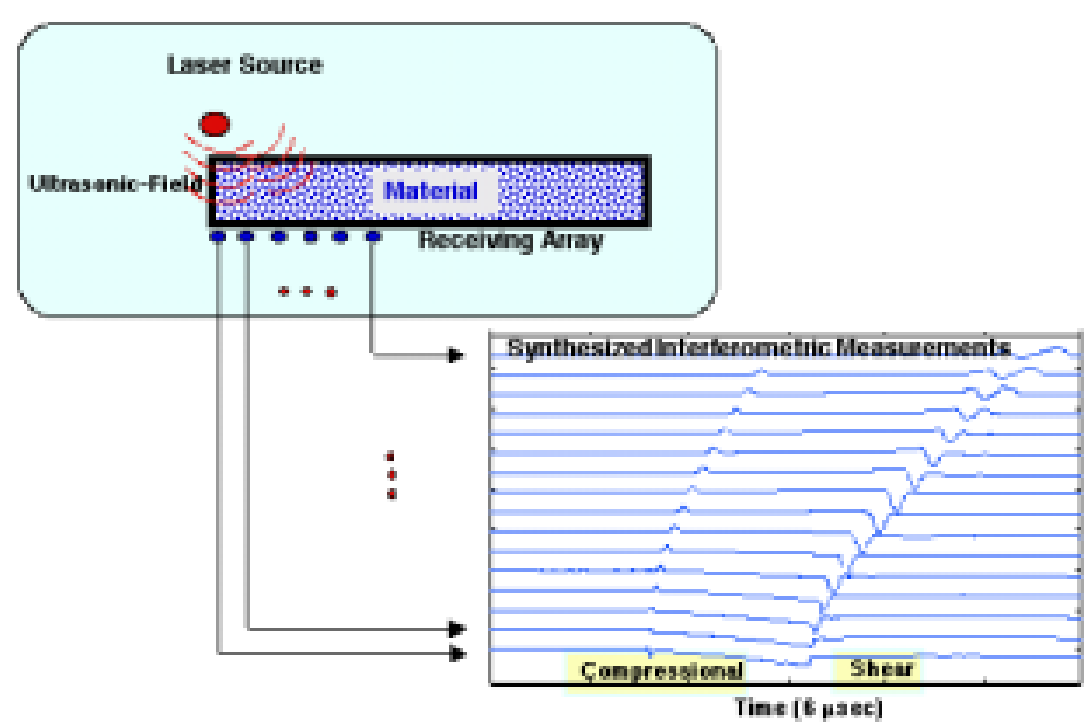

\section{PRE-PROCESSING}


S I G N A L / I M A G E P R O C E S S I N G F O R N D E

Critical preprocessing is accomplished using a correlation canceller algorithm to remove the "wash and primary arrivals"

Measurement

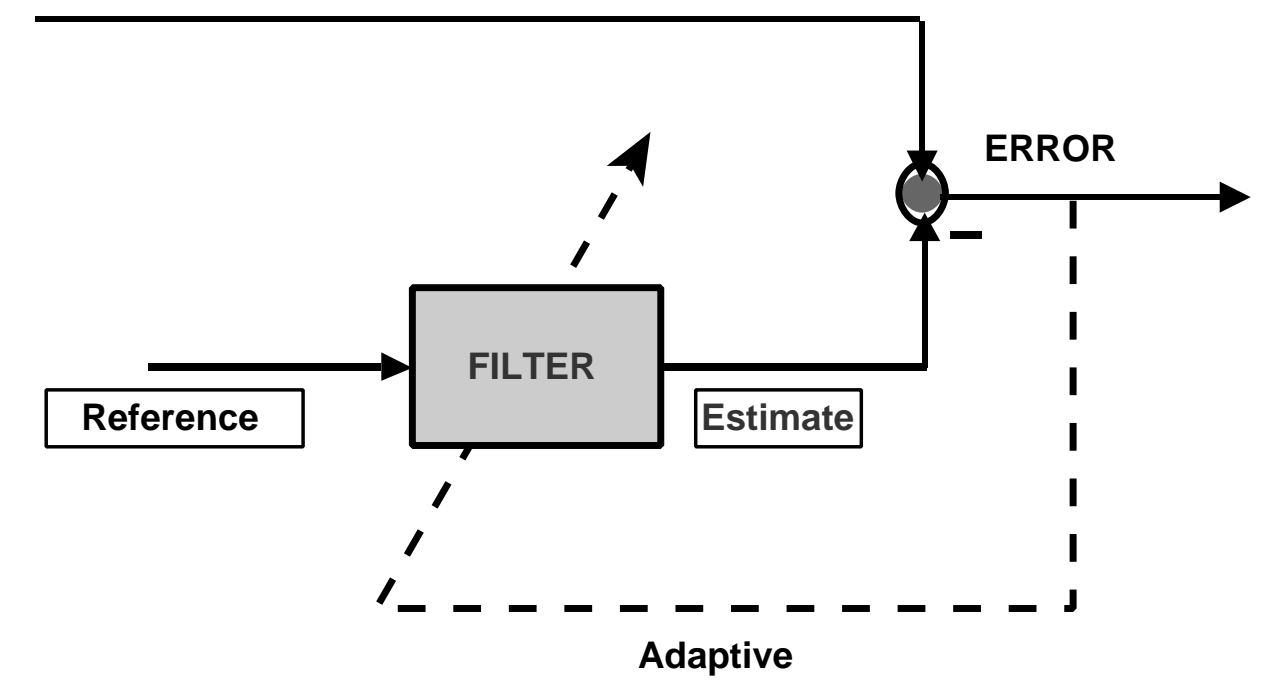

\section{CORRELATION CANCELLER}

MEASUREMENT + EXTRANEOUS DISTURBANCE +NOISE

$\mathrm{y}(\mathrm{t})=\mathrm{s}(\mathrm{t})+\mathrm{e}(\mathrm{t})+\mathrm{n}(\mathrm{t})$

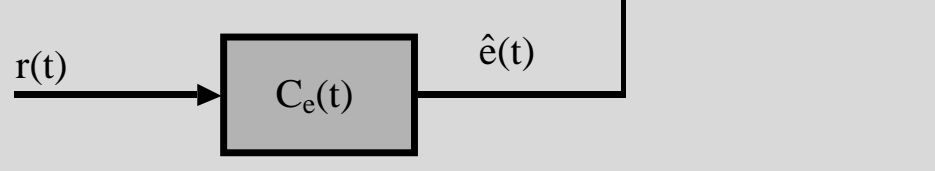

$$
\begin{aligned}
& e(t)=y(t)-\hat{\mathrm{e}}(t)=[s(t)+\mathrm{e}(t)+n(t)]-\hat{\mathrm{e}}(t) \gg s(t) \\
& \hat{\mathrm{e}}(t)=C_{\mathrm{e}}(t)^{*} r(t) \\
& \underline{C}_{\mathrm{e}}=R_{r r}^{-1} \underline{r}_{y r}
\end{aligned}
$$


S I G N A L / I M A G E P R O C E S S I N G F O R N D E

\section{CORRELATION/NOISE CANCELLATION: is a form of optimal mean-squared error estim.}

Suppose a measurement can be decomposed into two parts: one correlated and one uncorrelated

$$
y(t)=y_{-i}(t)+y_{\text {-it }}(t)
$$

where $y_{\infty}(t)\left[Y(t-1) \quad\right.$ [past data] and $y_{-m}(t) \perp Y(t-1) \quad$ [past data]

For optimal estimation $\hat{y}(t) \ldots y_{-}(t)$ and therefore, we define the error' as

\begin{tabular}{|c|c|}
\hline$e(t) \ldots y(t)-\hat{y}(t)$ & $y_{\ldots}(t)$ \\
\hline
\end{tabular}

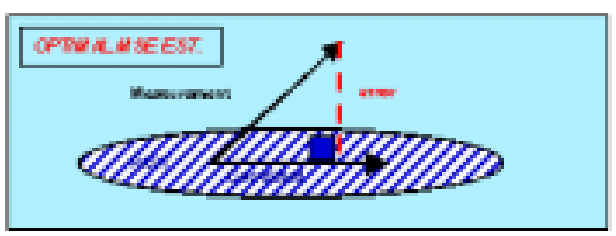

\section{Correlation cancelling applied to experimental data has proven to be vital to remove the "wash and primary arrivals"}

Laser Ultrasonic Correlation Cancellation

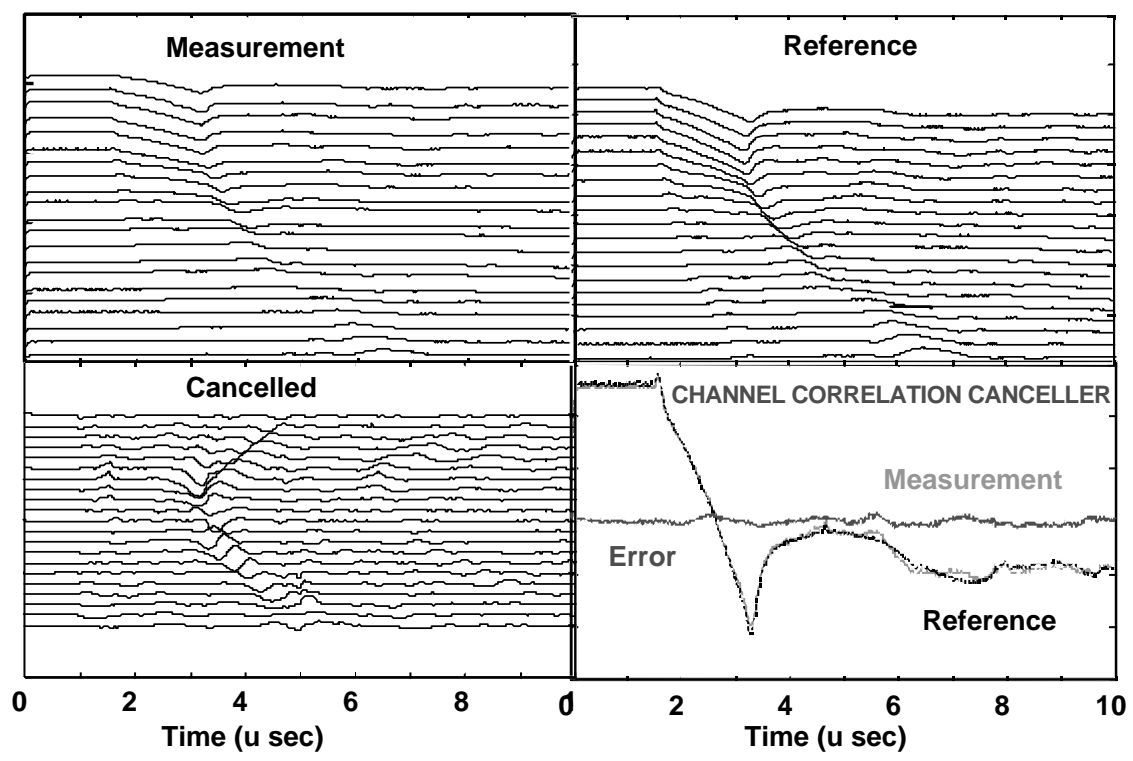




\section{SUMMARY}

We have developed a novel correlation canceling approach to remove unwanted components of signals, and have applied it to actual experimentally obtained laser-based ultrasound signals.

LAWRENCE LIVERMORE NATIONAL LABORATORY 
S I G N A L / I M A G E P R O C E S S I N G F O R N D E

\section{MATCHED-FIELD IMAGING \\ of \\ ULTRASOUND \\ for \\ NONDESTRUCTIVE EVALUATION}

J. Candy, R. Huber, D. Chambers, G. Thomas

CASIS Workshop

Livermore, CA

November 11-12, 1999

LAWRENCE LIVERMORE NATIONAL LABORATORY

ORGANIZATION

- INTRODUCTION:

- MATCHED-FIELD Imaging

- Results

- Summary

LAWRENCE LIVERMORE NATIONAL LABORATORY 
S I G N A L / I M A G E P R O C E S S I N G F O R N D E

\section{MATCHED-FIELD IMAGING APPROACH}

\section{MATCHED-FIELD IMAGING is:}

- Is a technique to detect, localize and image flaws for NDE

- Is the optimal solution to a basic "flaw detection" problem where a known (or modeled) field is used

- Is the "matched-filter" solution to a known signal in noise

- The extension of matched-field processing (ocean acoustics) to the ultrasonic imaging problem

- Is a generalization of the Synthetic Aperture Focusing Technique (SAFT) approach to imaging 
S I G N A L / I M A G E P R O C E S S I N G F O R N D E

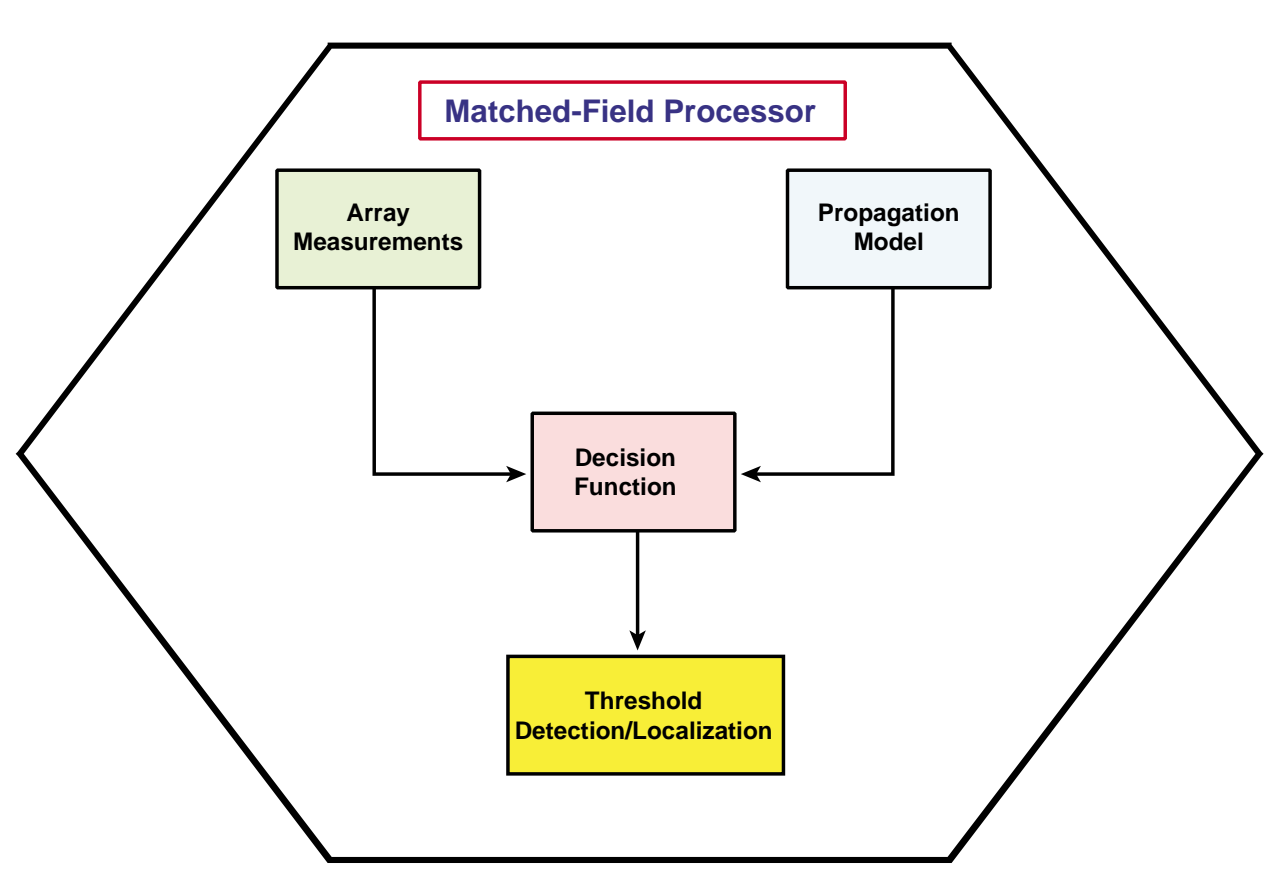

LAWRENCE LIVERMORE NATIONAL LABORATORY

When the position of the "flaw" is unknown, we must perform a composite hypothesis test to solve the detection problem, i.e. ,

The composite hypothesis test is:

$\begin{array}{llc}\mathrm{H}_{0}: & \underline{\mathrm{d}}(\mathrm{t})=\underline{\mathrm{n}}(\mathrm{t}) & {[\text { noise only }]} \\ \mathrm{H}_{1}: & \underline{\mathrm{d}}(\mathrm{t})=\underline{\mathrm{m}}(\mathrm{t} ; \theta)+\underline{\mathrm{n}}(\mathrm{t}) . & {[\text { signal }+ \text { noise }]}\end{array}$

which leads to a generalized likelihood ratio test which can be summarized by:

\begin{tabular}{|c|c|}
\hline $\max _{\theta_{\mathrm{s}}} \mathrm{P}\left(\underline{\boldsymbol{\theta}}_{\mathrm{S}}\right)$ & $\begin{array}{l}\stackrel{\mathrm{H}_{1}}{>} \\
\stackrel{\mathrm{H}_{0}}{<}\end{array} \lambda_{\theta}$ \\
\hline \multicolumn{2}{|l|}{ where } \\
\hline & $\left\langle\underline{\mathrm{m}}^{\prime}\left(\mathrm{T} ; \underline{\theta}_{\mathrm{S}}\right) * \underline{\mathrm{d}}(\mathrm{T})^{2}\right\rangle$ \\
\hline & $\underline{\mathrm{m}^{\prime}}\left(\mathrm{T} ; \theta_{\mathrm{s}}\right) * \mathrm{~m}\left(\mathrm{TT} ; \theta_{\mathrm{s}}\right)$ \\
\hline
\end{tabular}

LAWRENCE LIVERMORE NATIONAL LABORATORY 
S I G N A L / I M A G E P R O C E S S I N G $\quad F \quad O \quad R \quad$ N D E

The signal model that we developed is the geometric optics simplification evolving from the elastic wave equation

The model has both compressional and shear components

$$
\underline{f}(\mathrm{t})=\underline{\mathrm{G}}_{\mathrm{c}}(\mathrm{t})^{*} \mathrm{~s}_{\mathrm{c}}(\underline{\mathrm{r}}, \mathrm{t})+\underline{\mathrm{G}}_{\mathrm{s}}(\mathrm{t})^{*} \mathrm{~s}_{\mathrm{s}}(\underline{\mathrm{r}}, \mathrm{t})
$$

which can be simplified significantly in a homogeneous medium to:

$$
\underline{\mathrm{m}}(\mathrm{T})=\underline{f}(\mathrm{~T}-\mathrm{t})=\underline{\mathrm{G}}_{\mathrm{c}}(\mathrm{T}-\mathrm{t})+\underline{\mathrm{G}}_{\mathrm{s}}(\mathrm{T}-\mathrm{t})
$$

\section{NOTE:}

- $\quad$ This is the time reversed, matched-filter solution

Ignoring either the compressional or shear velocity, we obtain the SAFTsolution

${ }^{\star * * *}$ A CONVENTIONAL SUM-DELAY BEAMFORMER ****

LAWRENCE LIVERMORE NATIONAL LABORATORY

The "matching function" is generated from a propagation model which we generate from the geometric optics viewpoint

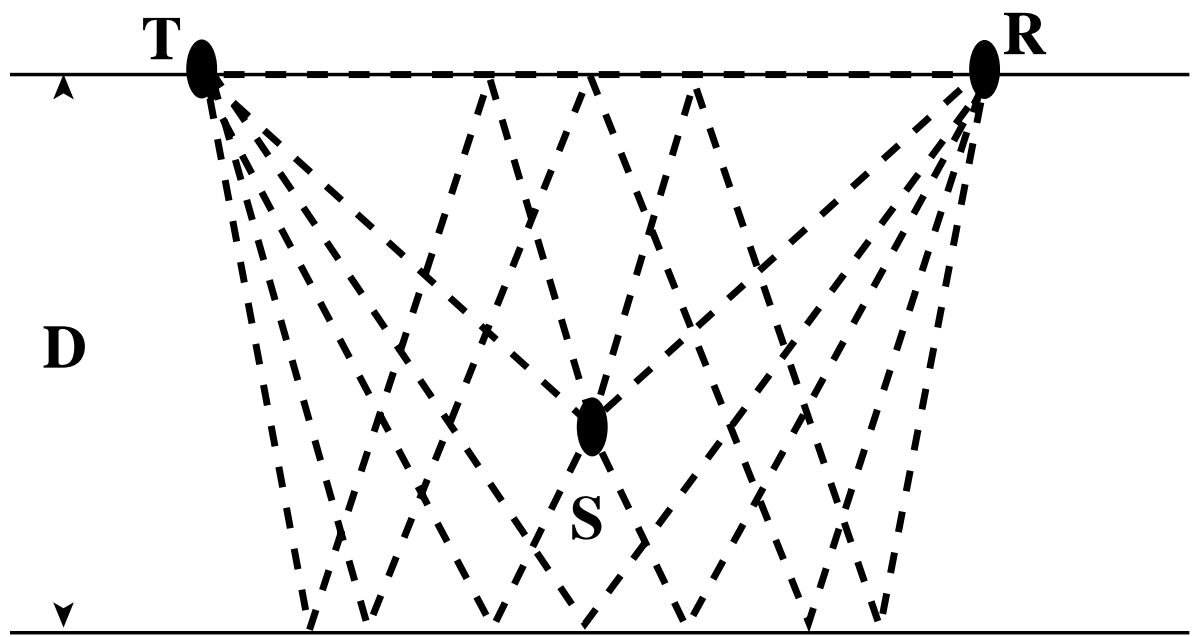

LAWRENCE LIVERMORE NATIONAL LABORATORY 
S I G N A L / I M A G E P R O C E S S I N G F O R N D E

A typical geometric optics simulation of an aluminum part of $9.5 \mathrm{~mm}$ in width and $20 \mathrm{~mm}$ in length is given by:

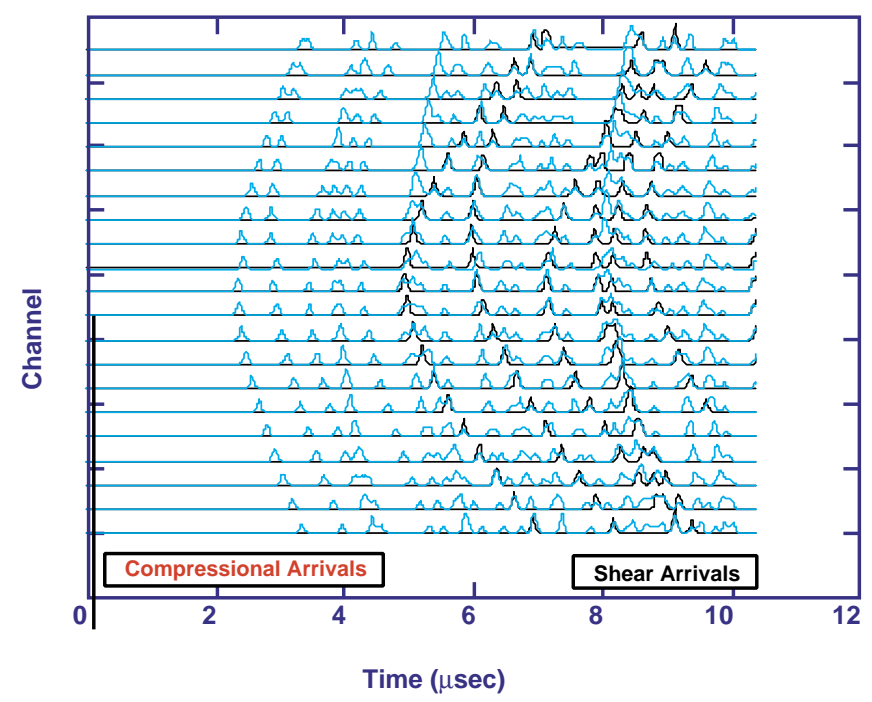

LAWRENCE LIVERMORE NATIONAL LABORATORY

The Optimal Matched-Field Imaging ALGORITHM is:

- Decompose the test specimen into rectangular pixels specified by the desired spatial sampling intervals, $\left(D_{x}, D_{y}\right)$

- For each pixel generate the model replicant by backpropagating the field to the array at each sensor position using the propagation model

- Perform the required convolutions, squaring and summing operations

- Threshold filter the image so that the maximum threshold is satisfied

- Search for maxima to "detect" and "localize" flaws

\section{LAWRENCE LIVERMORE NATIONAL LABORATORY}



S I G N A L / I M A G E
P R O C C E S S S I N $\mathrm{N}$
F O R
N D E

Applying the "matching function" generated from the geometric optics propagation model shows the optimal solution at the flaw

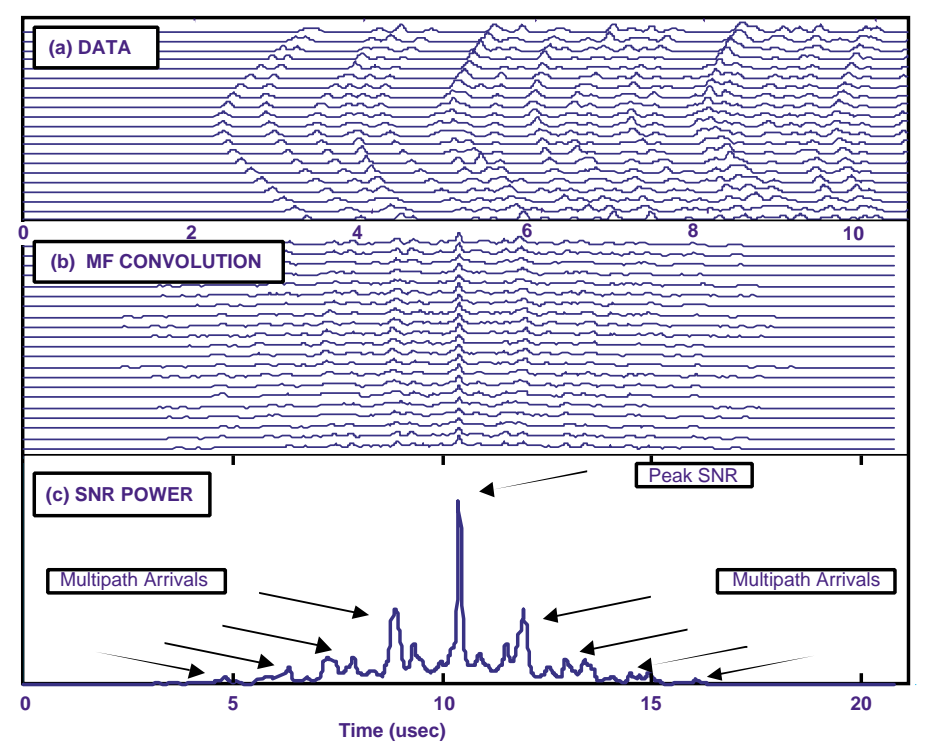

LAWRENCE LIVERMORE NATIONAL LABORATORY

The "optimal" MFI processor works well on simulated data with flaws at $(10 \mathrm{~mm}, 5 \mathrm{~mm})$ and $(12 \mathrm{~mm}, 3 \mathrm{~mm})$ using the multipath model.

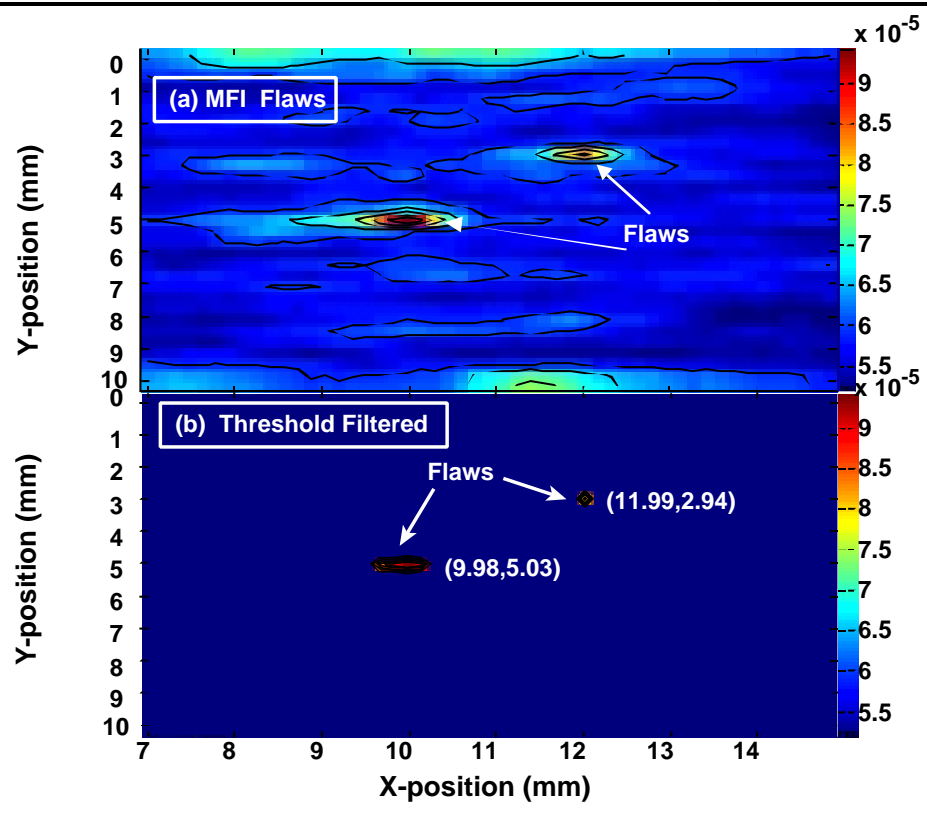

LAWRENCE LIVERMORE NATIONAL LABORATORY 
S I G N A L / I M A G E P R O C E S S I N G F O R N D E

The "SUBoptimal" SAFT processor performs reasonably on the simulated data with flaws at $(10 \mathrm{~mm}, 5 \mathrm{~mm})$ and $(12 \mathrm{~mm}, 3 \mathrm{~mm})$ using a homogeneous model without multipath.

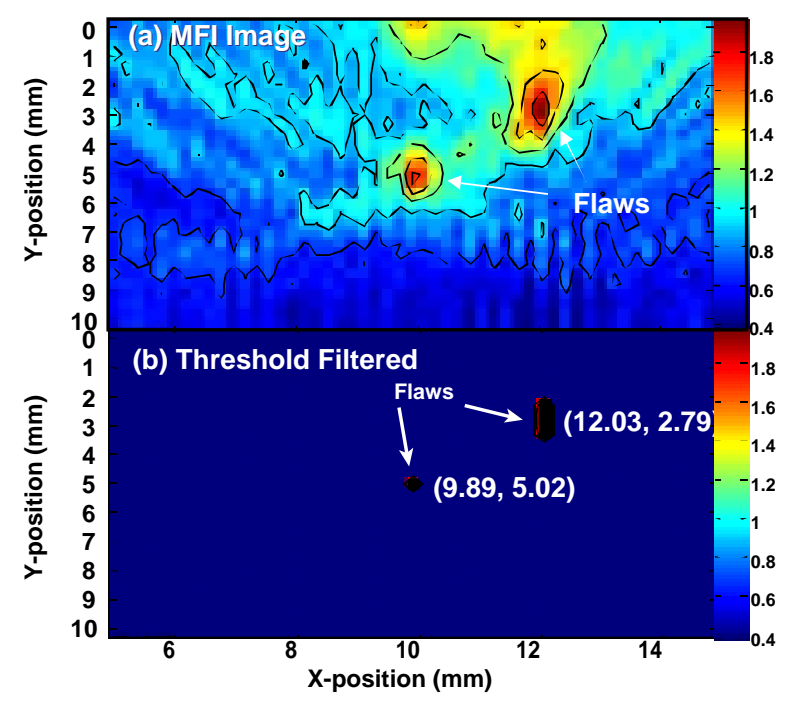

LAWRENCE LIVERMORE NATIONAL LABORATORY

\section{RESULTS}



S I G N A L / I M A G E
P R O C E S S I N G
F O R
N D E

Epicentral scans of an aluminum part of $9.5 \mathrm{~mm}$ in width and $20 \mathrm{~mm}$ in length with a hole at $(10 \mathrm{~mm}, 5 \mathrm{~mm})$ provide a flaw detection

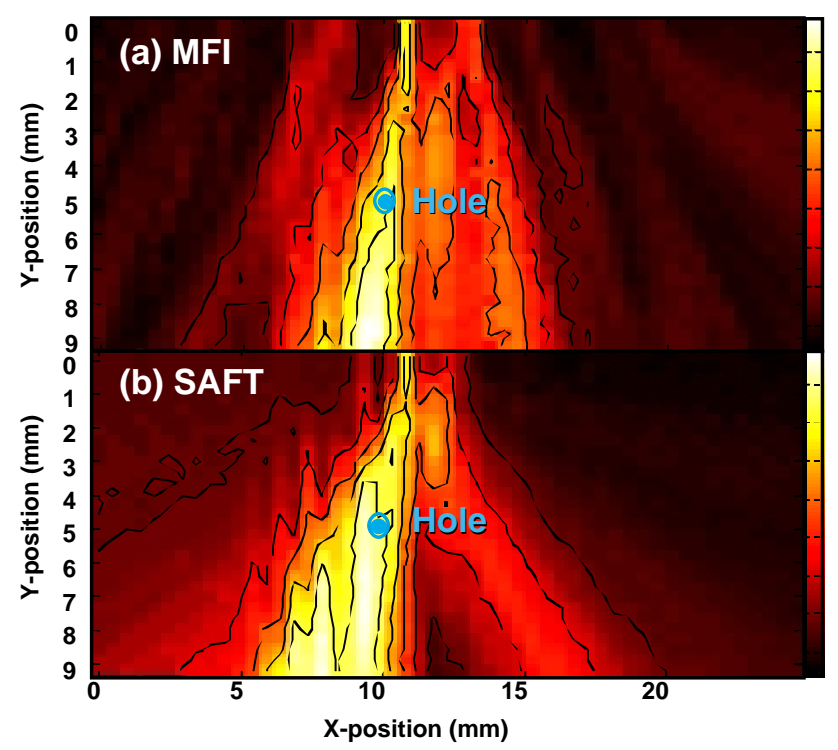

LAWRENCE LIVERMORE NATIONAL LABORATORY

Epicentral scans of an aluminum part of $9.5 \mathrm{~mm}$ in width and $20 \mathrm{~mm}$ in length also detect flaws (holes) at (10 $\mathrm{mm}, 5 \mathrm{~mm})$ and $(12 \mathrm{~mm}, 3 \mathrm{~mm})$

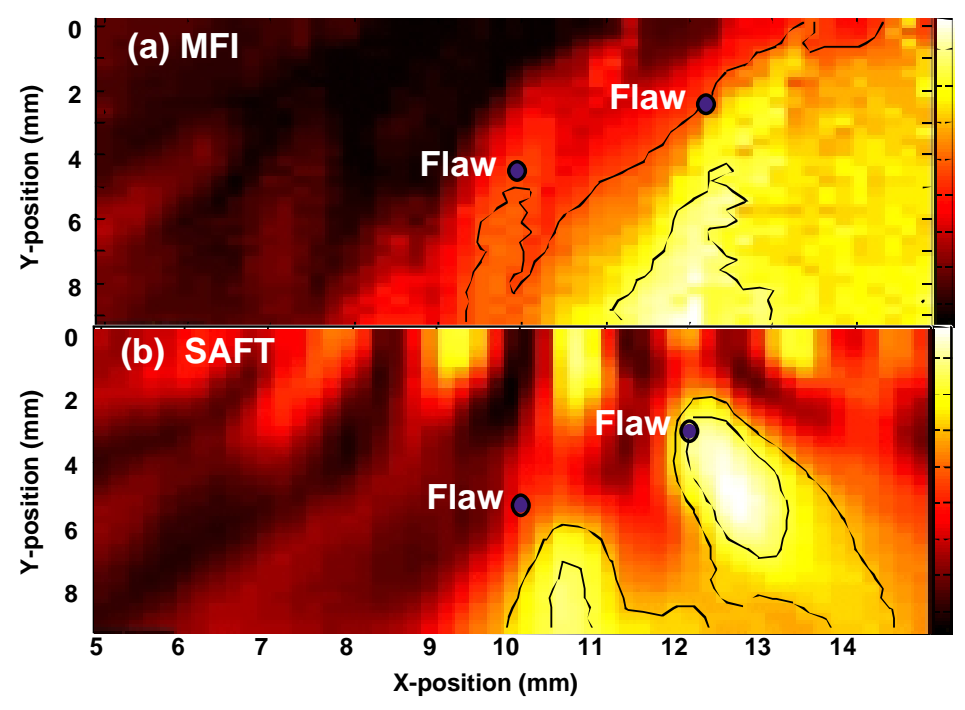

LAWRENCE LIVERMORE NATIONAL LABORATORY 

S I G N A L / I M A G E
P R O C E S S I N G
F O R
N D $E$

A decoupage of images, one for each source location, provides
an evolution of the flaw detection and MFI Shear Only process
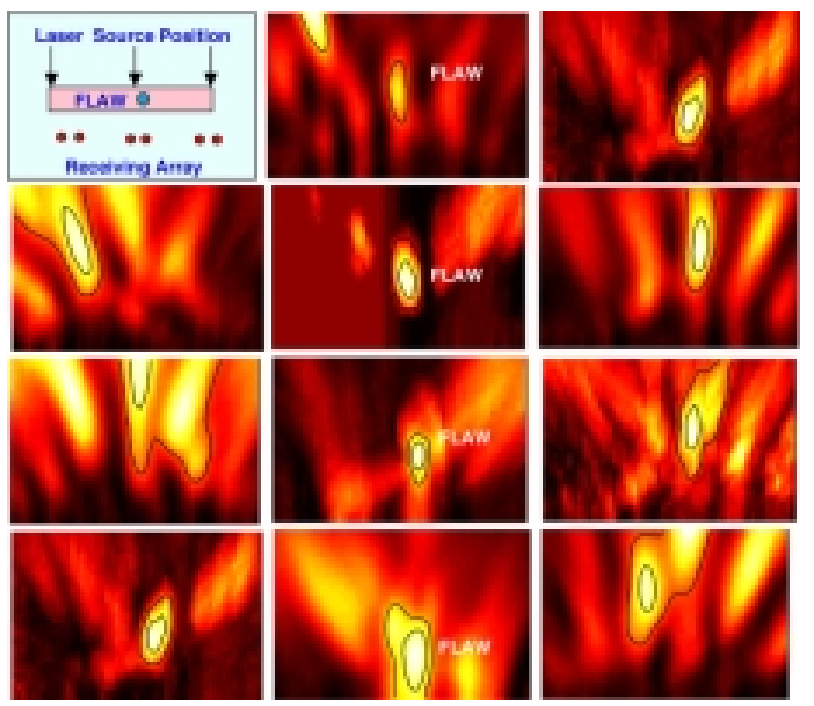

MFI LASER ULTRASONIC IMAGING (Shear)

LAWRENCE LIVERMORE NATIONAL LABORATORY

A decoupage of images, one for each source location, provides an evolution of the flaw detection and MFI homogenous process
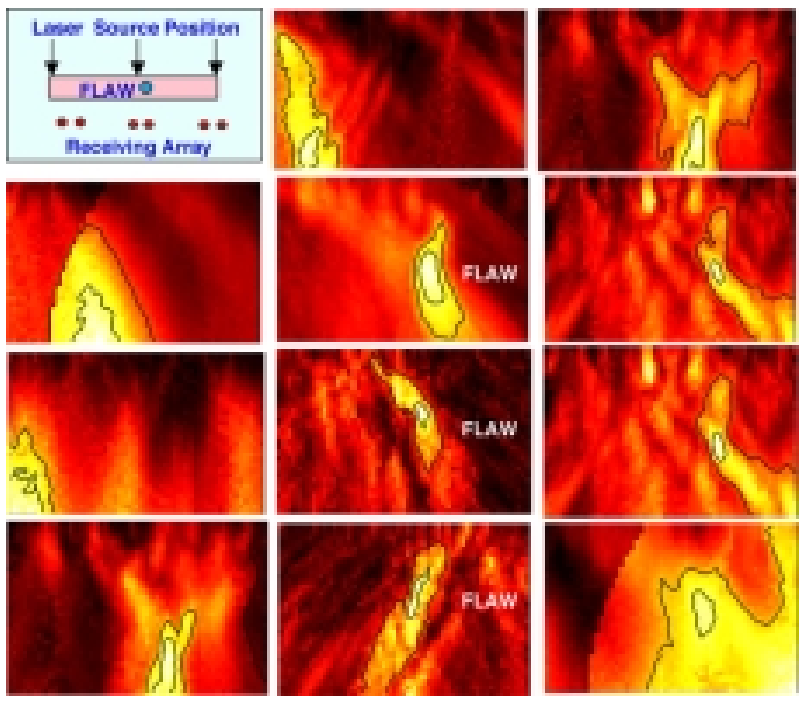

LAWRENCE LIVERMORE NATIONAL LABORATORY 
S I G N A L / I M A G E P R O C E S S I N G F O R N D E

\section{SUMMARY}

We have developed a "matched-field imaging" approach to imaging laser ultrasonic data obtaining by creating a synthetic spatio-temporal array matched filter processing using a multipath, homogeneous

medium model demonstrating the feasibility of such an approach on both simulated and experimental data

LAWRENCE LIVERMORE NATIONAL LABORATORY 


\title{
Void Analysis from CT Imagery with Applications to Damage Evolution in an AM60B Magnesium Alloy
}

\author{
Amy M. Waters, Harry E. Martz, Kenneth W. Dolan, \\ Mark F. Horstemeyer ${ }^{2}$, Earl O. Updike, and Robert E. Green, Jr. ${ }^{3}$
}

Lawrence Livermore National Laboratory (LLNL) and Sandia National Laboratory (SNL) are collaborating on the development of new techniques to study damage evolution and growth in material specimens subjected to mechanical testing. These techniques include metallography, radiography, computed tomography (CT) and modeling. The material specimens being studied include cast magnesium and aluminum alloys, and forged stainless steel. We will concentrate on characterizing mechanically deformed magnesium alloy specimens using computed tomography. Several notched tensile magnesium specimens were uniaxially loaded to obtain different levels of deformation. Specimens were initially characterized by radiography and computed tomography to determine the preloaded state. Subsequent CT scans were performed at different displacement percentages. The CT volumetric data are being used to measure void volume fraction in all three dimensions nondestructively to determine the effect of void growth on the mechanical behavior of the materials. 
S I G N A L / I M A G E P R O C E S S I N G F O R N D E

\title{
Void Analysis from CT Imagery with Applications to Damage Evolution
}

\author{
prepared by \\ Amy M. Waters, Harry E. Martz, \\ Ken Dolan, and Derrill Rikard \\ Nondestructive Evaluation Section \\ Lawrence Livermore National Laboratory \\ Mark Horstemeyer \\ Sandia National Laboraties, California \\ Robert E. Green, Jr. \\ The Johns Hopkins University \\ presented at \\ CASIS Workshop \\ LLNL
}

November 11 - 12, 1999

Livermore, California

\section{Agenda}

- Background of project

- Overview of thesis research

- Magnesium alloy tensile bars

- Mechanical loading

- CT simulation studies

- CT data acquisition

- CT data processing

- Preliminary results

- Void analysis

- Summary and future work

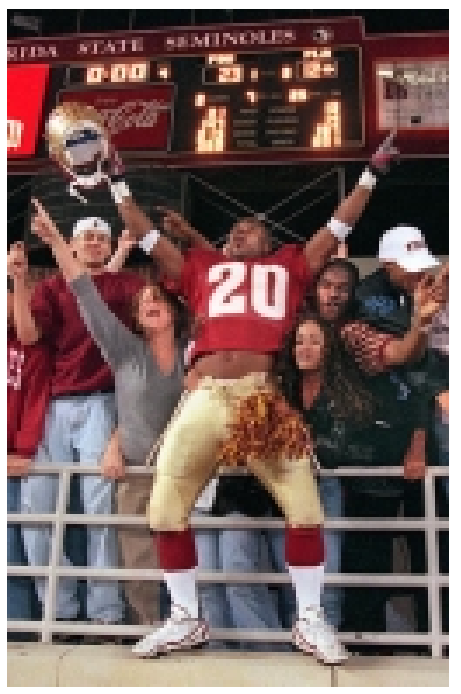



S I G N A L / I M A G E
P R O C C E S S S I N G
F O R
N D E

\section{We are using CT to help develop cast light metals for automotive components (Sponsor: USAMP)}

- Sponsor: United States Automotive Materials Partnership (USAMP), Lawrence Livermore National Laboratory, and the Center for Nondestructive Evaluation, Johns Hopkins University

- Design and Product Optimization for Cast Light Metals

- Microstructure Characterization - Arun Gokhale, Georgia Institute of Technology

- Solidification Model - S. Viswanathan, Oak Ridge National Laboratory

- Database - Jim Sudy, Westmoreland Mechanical Testing and Research

- Validation - All team members

- Crack Nucleation - Jinghong Fan and David McDowell, Georgia Tech

- Property Model - Mark Horstemeyer, Sandia National Laboratory

- Design Guide - Greg Sanders, Entelechy

- Monitoring/Quality Assurance - Ken Dolan, LLNL

\section{Microstructure Characterization: goals/objectives}

- Input initial microstructural state (porosity, etc.) of castings into material model and perform structural analysis (FEM) on castings to simulate in-service performance

- Characterize behavior of Mg-alloys

- Characterize internal structure, e.g., defects, voids, inclusions, cracks

- Create material models to predict behavior based on internal structure perform finite element modeling based on material models and structure

- Validate models

- Mechanical testing 

S I G N A L / I M A G
$P$
O C E S S I N G
F O R
N D E

\section{Summary of the steps required to characterize and}

eventually predict the behavior of new materials

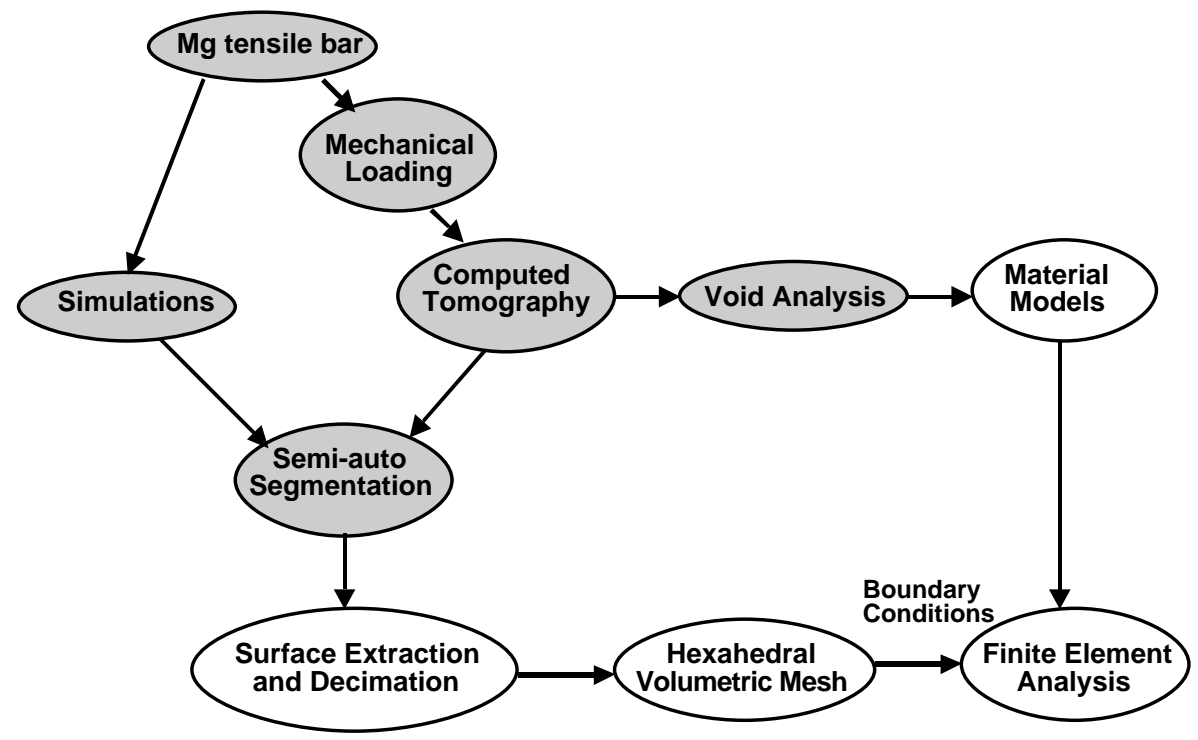

\section{We are studying 9 magnesium alloy tensile specimens}

- Mg-alloy is AM60B containing $6 \%$ aluminum and $0.377 \%$ manganese by weight

- Samples were cast using a cold chamber die casting machine with a 600 -ton locking force, injection temperature of 675 to 690 celsius

- Three different notch geometries, Series F, G, H

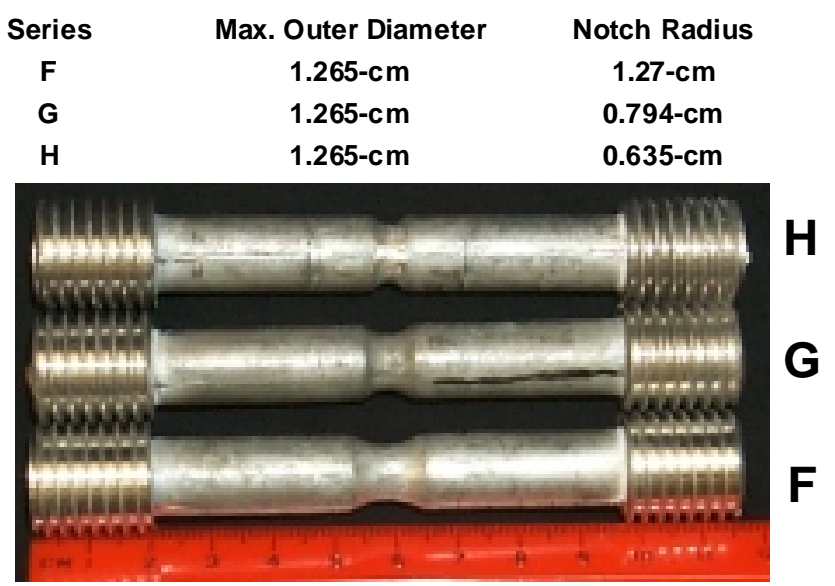


S I G N A L / I M A G E P R O C E S S I N G F O R N D E

We are using CT throughout the mechanical loading of the tensile bars to better understand the dynamics of damage evolution

- Samples will be loaded to percentages of the failure load

$-60 \%, 87 \%, 93 \%$ and $100 \%$

- Failure loads were detemined by Westmoreland Mechnical Testing, PA

\begin{tabular}{cccccc} 
Series & $\begin{array}{c}\text { Notch } \\
\text { Radius }(\mathrm{cm})\end{array}$ & $\begin{array}{c}\sigma_{\text {UTS }} \\
(\mathrm{MPa})\end{array}$ & $\begin{array}{c}60 \% \text { load } \\
(\mathrm{N})\end{array}$ & $\begin{array}{c}87 \% \text { load } \\
(\mathrm{N})\end{array}$ & $\begin{array}{c}93 \% \text { load } \\
(\mathrm{N})\end{array}$ \\
\hline H & 0.635 & 207 & 9176 & 13304 & 14225 \\
G & 0.794 & 221 & 9283 & 13460 & 14389 \\
F & 1.27 & 241 & 9879 & 14327 & 15314
\end{tabular}

We are using a radiographic simulation code (HADES) to optimize CT acquisition, data processing and analysis

HADES is a code developed at LLNL to simulate radiographs.

We are using HADES to model Mg-alloy tensile specimens with

KCAT scanner geometry and parameters.

Experimental radiograph

(75 KV w/0.08 mm Al filter)

Simulated radiograph (75 KV w/0.08 mm Al filter)

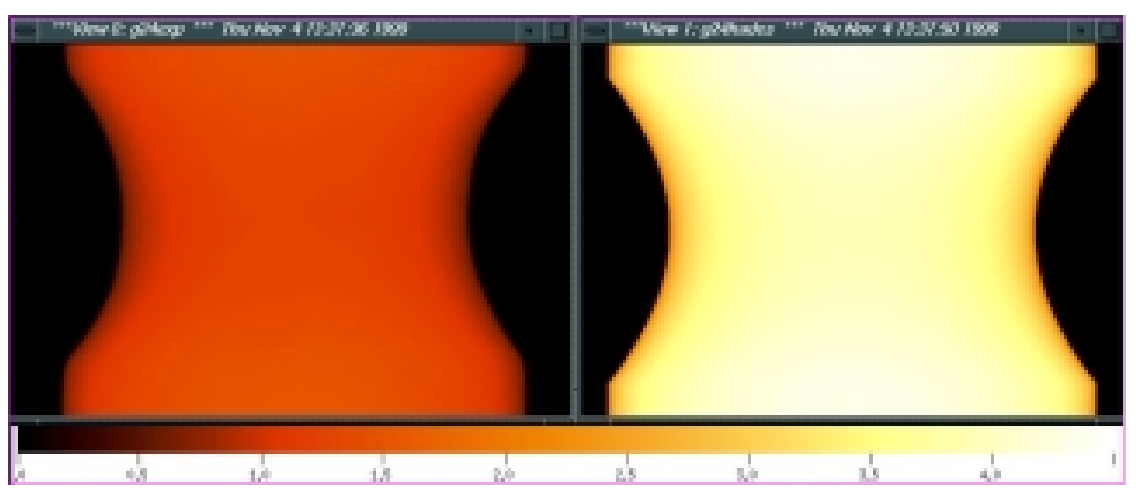



S I G N A L / I M A G E
P R O C E S S I N G
F O R
N D E

We have created a phantom made of pure magnesium (99.9\%) to study artifact reduction and to help optimize CT data acquisition

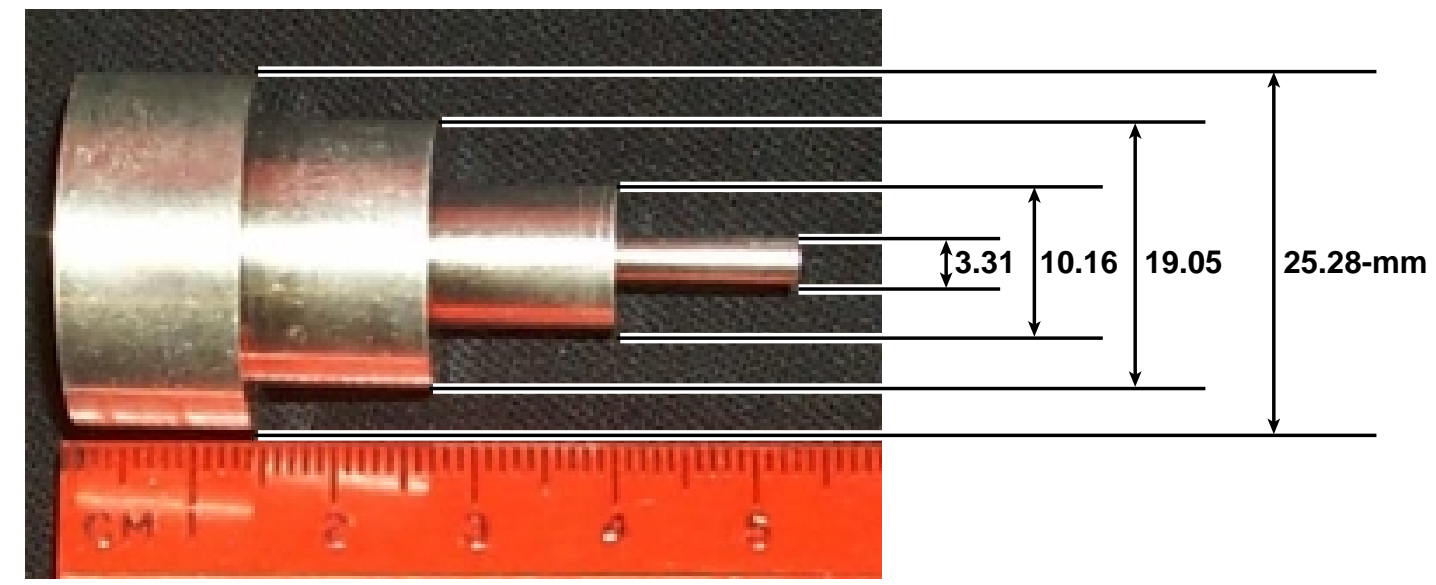

\section{We have simulated the pure Magnesium Phantom}

We will include voids and various objects in the simulated phantom to determine effect on $\mathrm{CT}$ reconstructions

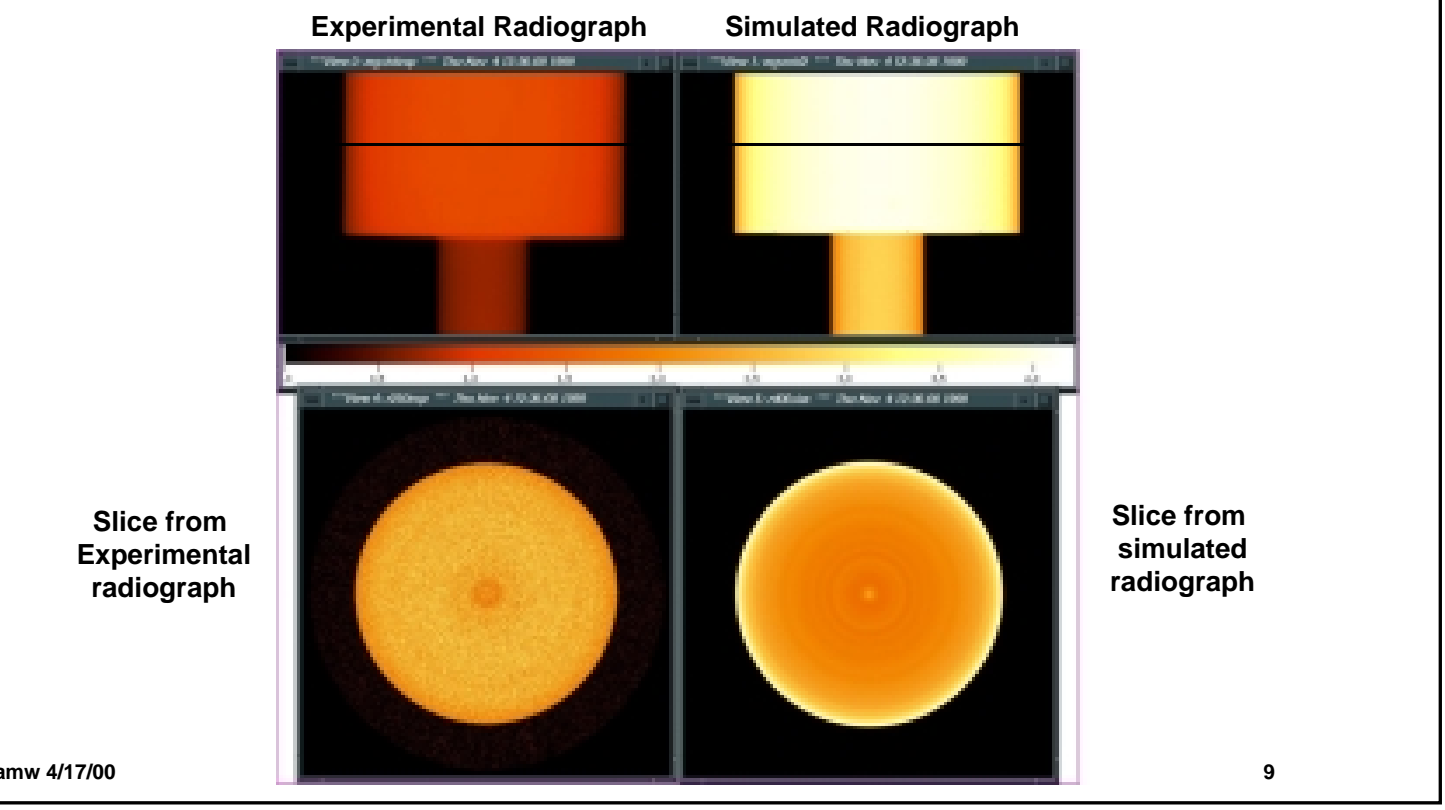



S I G N A L / I M
A G E
$\begin{array}{llll}P & R & O\end{array}$
C E S S S I N $\mathrm{N}$
F O R
N D E

We have researched, developed and applied several CT scanners including data processing and image reconstruction and analysis software

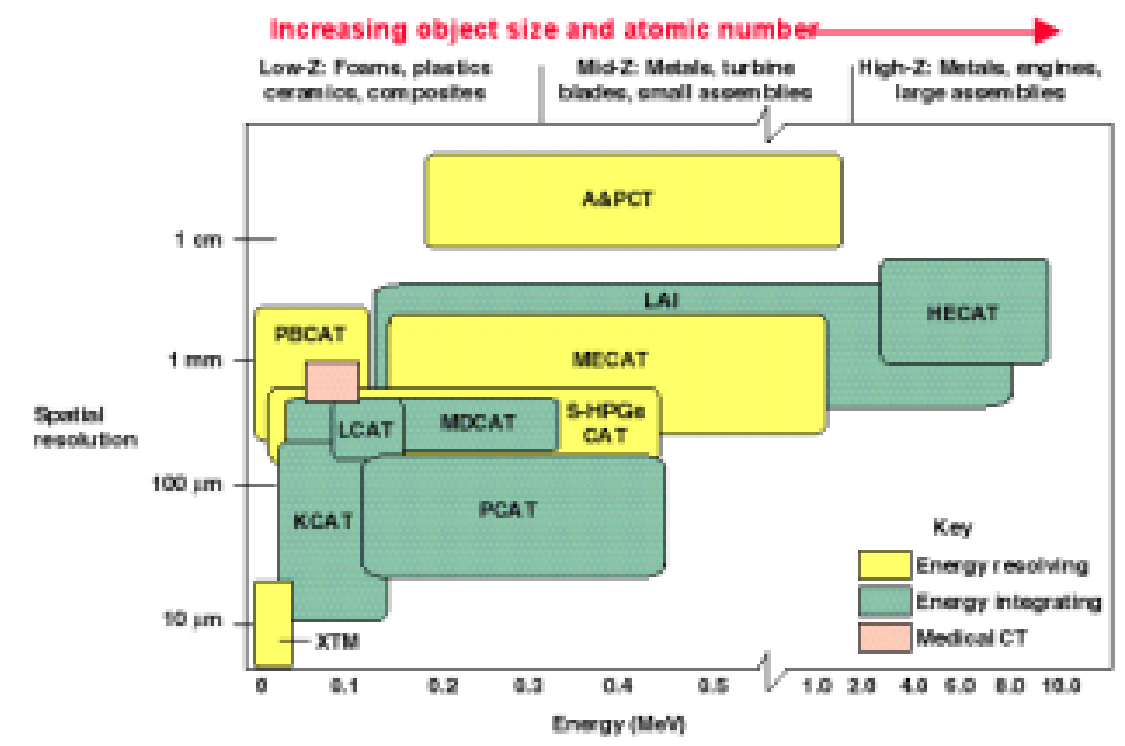

amw 4/17/00

We have used PCAT and KCAT to study the magnesium tensile bars

\section{Experimental Setup - KCAT}

KCAT is a high-spatial resolution CT scanner

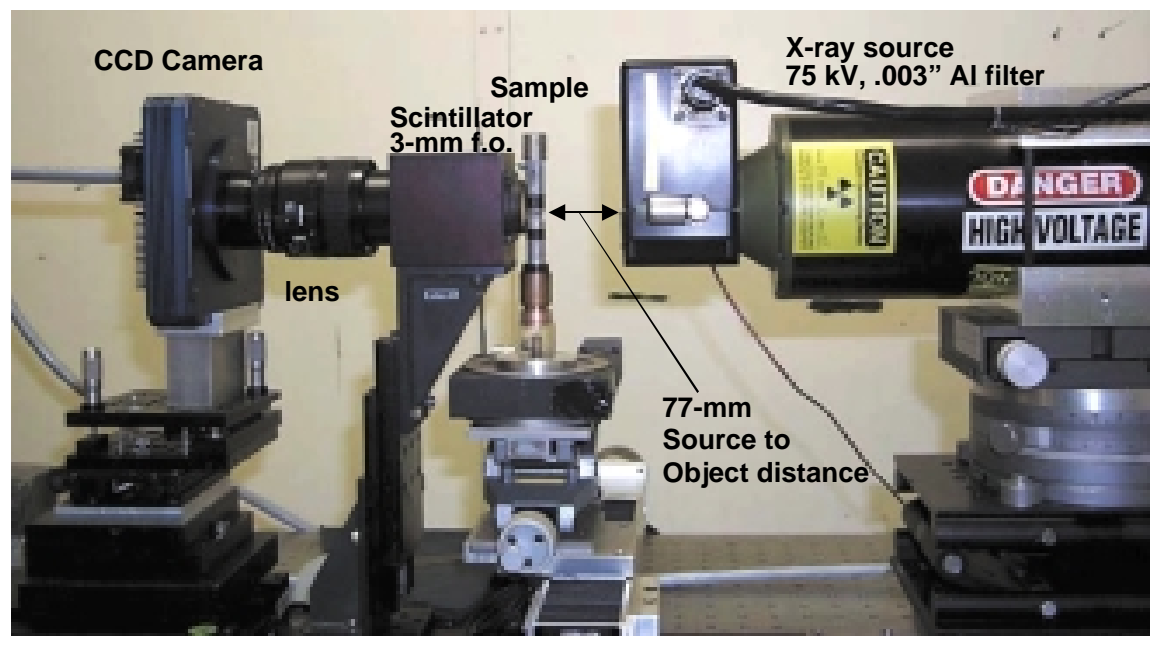

We acquired 360 projections over 360 degrees. Acquisition time was approximately 5 hours. Modulation of the system is approximately 0.5 at $10 \mathrm{lp} / \mathrm{mm}$. 
S I G N A L / I M A G E P R O C E S S I N G F O R N D E

\section{Schematic of scintillator/lens/CCD detector that we are using to study the magnesium tensile bars}

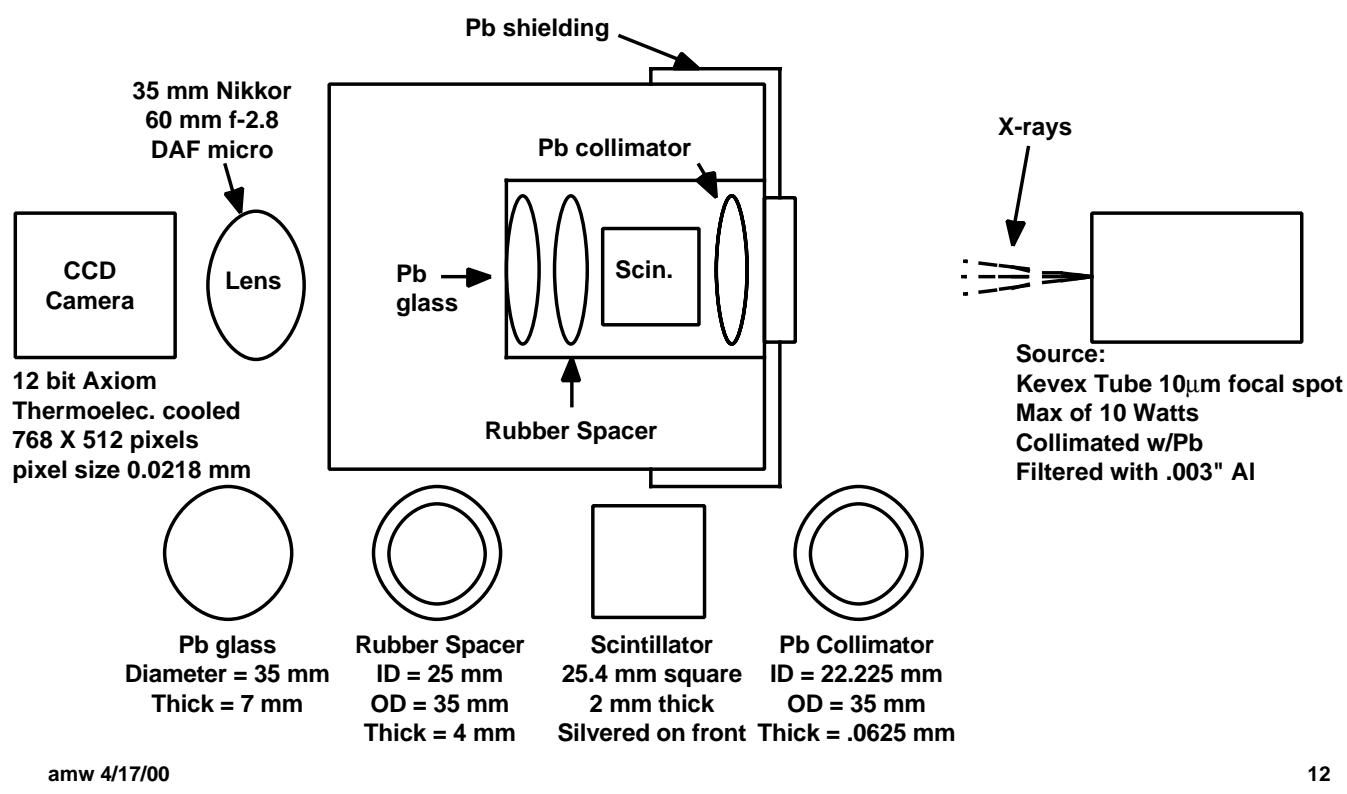

\section{Outlier Removal}

- An outlier removal routine is applied to all projections [In $\left.\left(I_{0} / l\right)\right]$ before processing

- A median filtered image is created and compared to original image. Any pixels with values outside a given difference are replaced by the median filterd value.

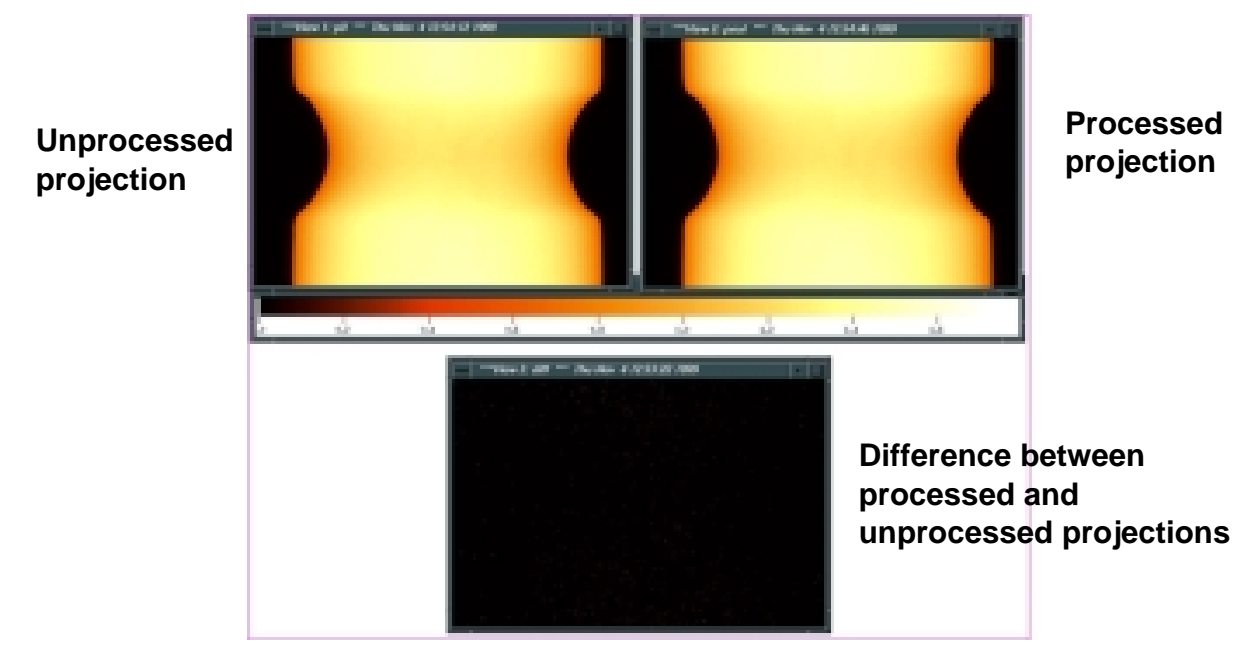



S I G N A L / I M A G E
P R O C E S S I N G
F O R
N D E

\section{Ring Removal}

Rings are due to an imbalance between detector elements. Sinograms were created and processed to removed ring artifacts. Rings appear as vertical lines in the sinogram and are minimized by using a combination of smoothing filters.

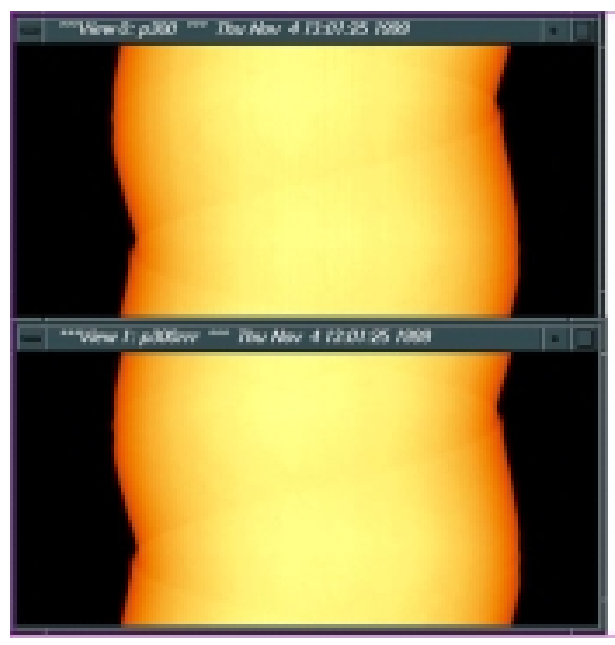

Unprocessed sinogram (with rings)

\section{Difference}

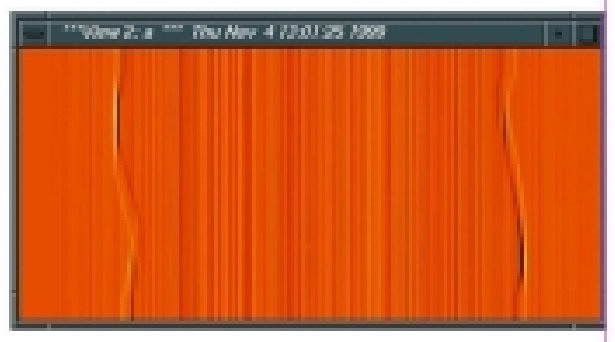

Processed sinogram (without rings)

\section{Ring removal helps simplify data interpretation and analysis}

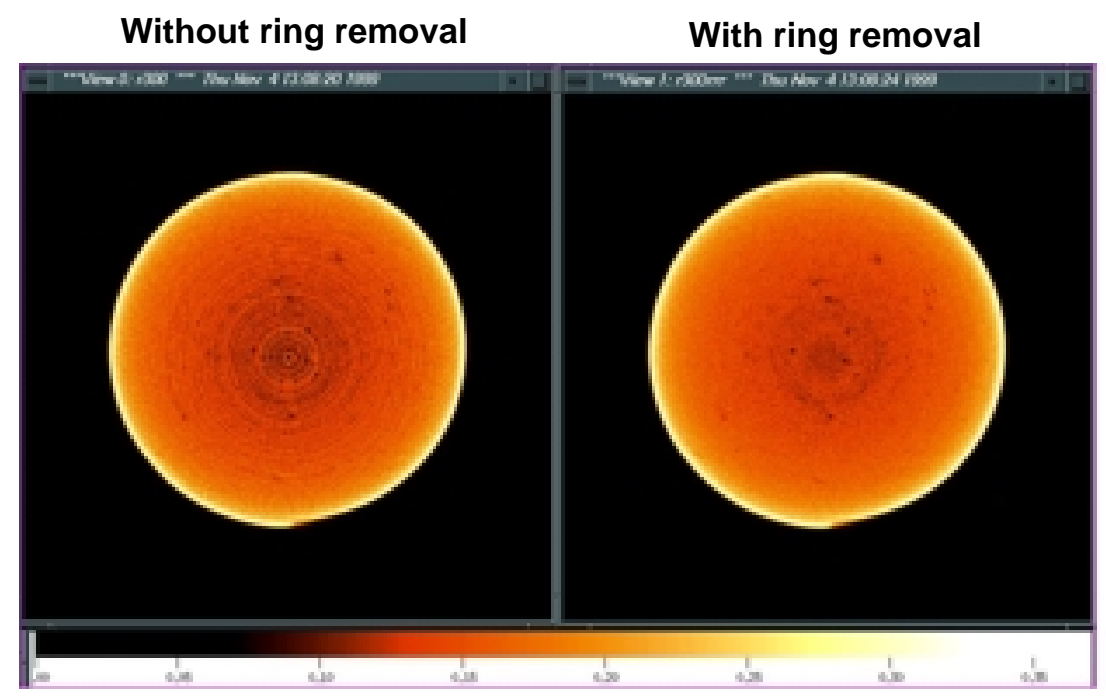



S
S I G N A L / I M A G E
P R O C E S S I N G
F O R
N D E

\section{Beam hardening is a result of the preferential absorption of low energy x-rays, resulting in a "cupping" artifact}

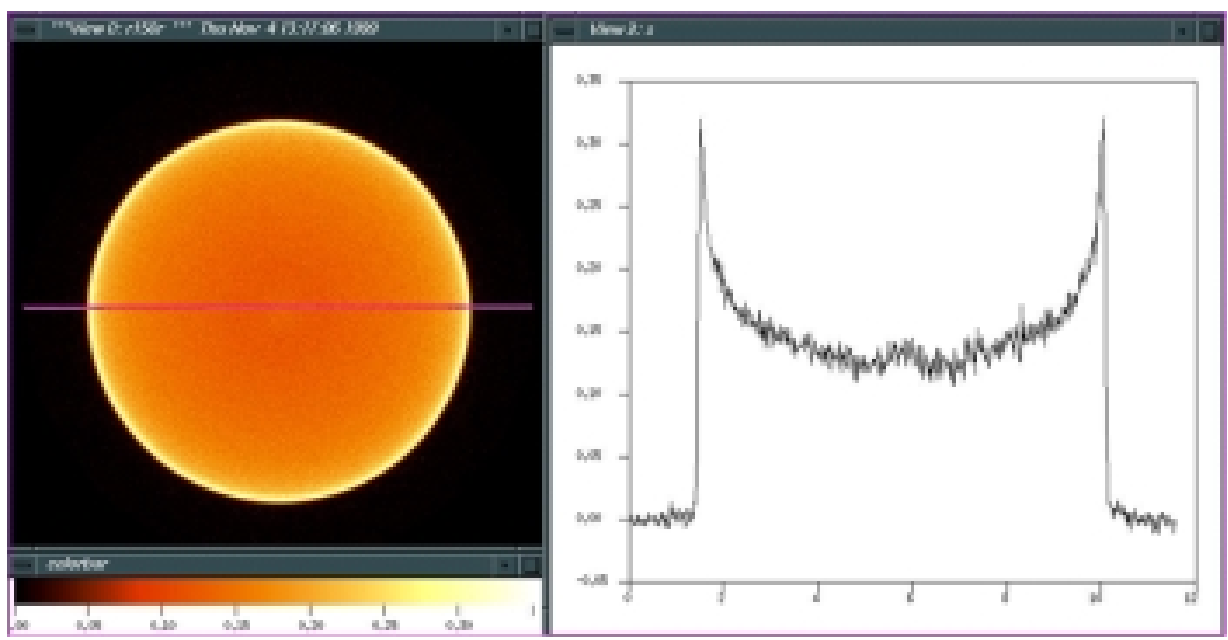

One reconstructed CT slice without any beam hardening corrections, and a 1-D profile. Note the apparent density gradient from outside to inside of part.

\section{Beam Hardening}
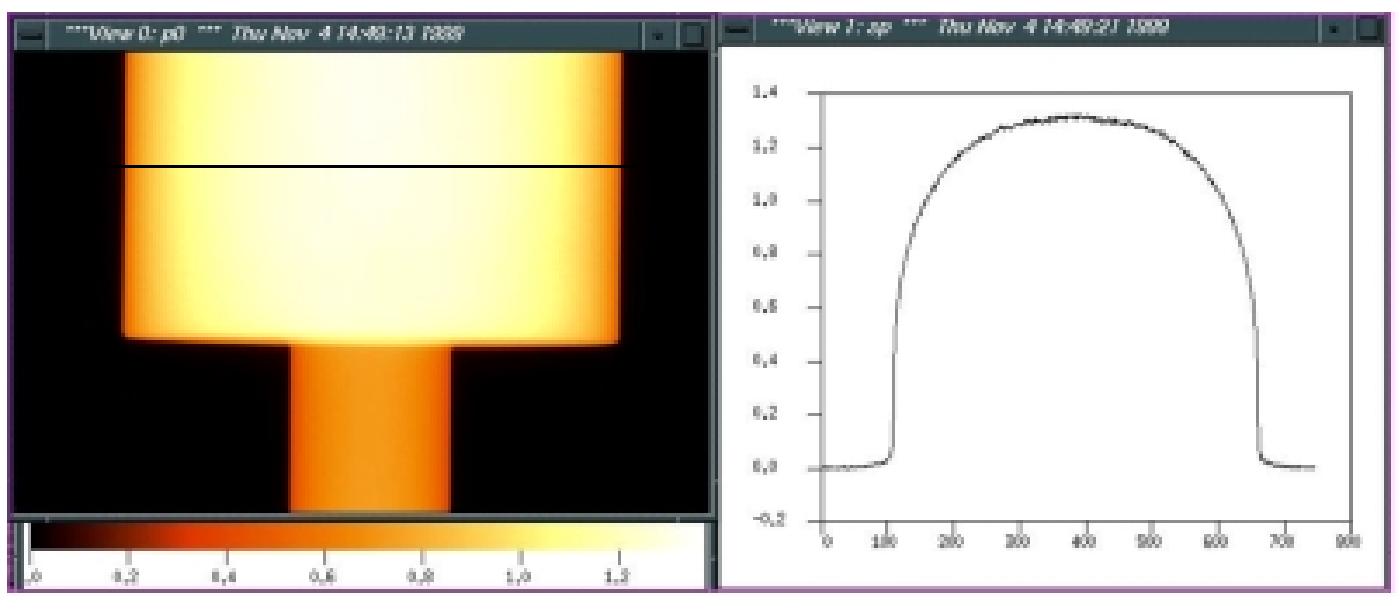

Radiograph of two smallest sections of Mg beam hardening phantom, 10.16 and 3.31-mm outer diameters.
One-dimensional profile from the larger section of $\mathrm{Mg}$ phantom. This data is used to obtain the function between chord length and attenuation times length $\left(\mu^{*}\right)$ 
S I G N A L / I M A G E P R O C E S S I N G F O R N D E

\section{Beam Hardening Correction}

Plot of attenuation vs. chord length. The best fit polynomial is used on the original sinogram to correct for beam hardening.

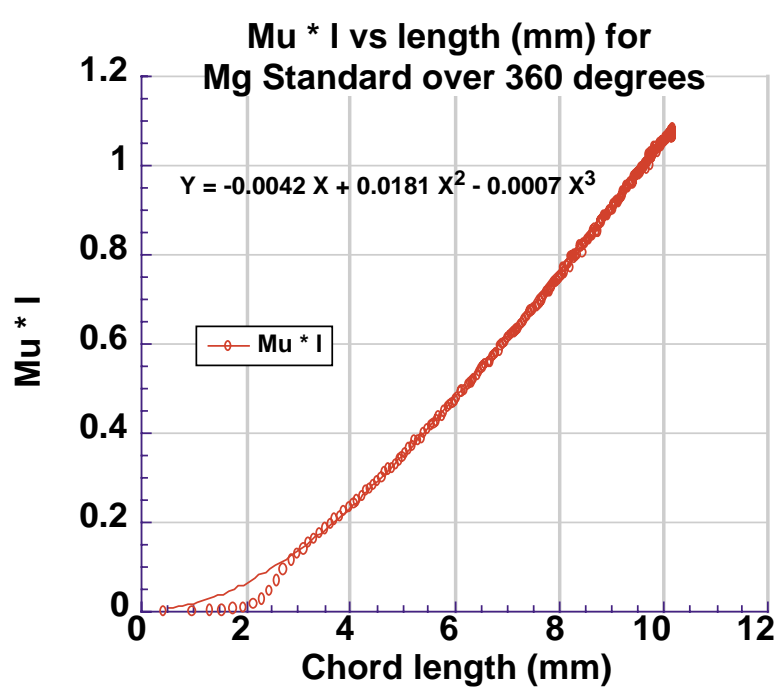

\section{Beam Hardening Correction}

No beam hardening correction With beam hardening correction

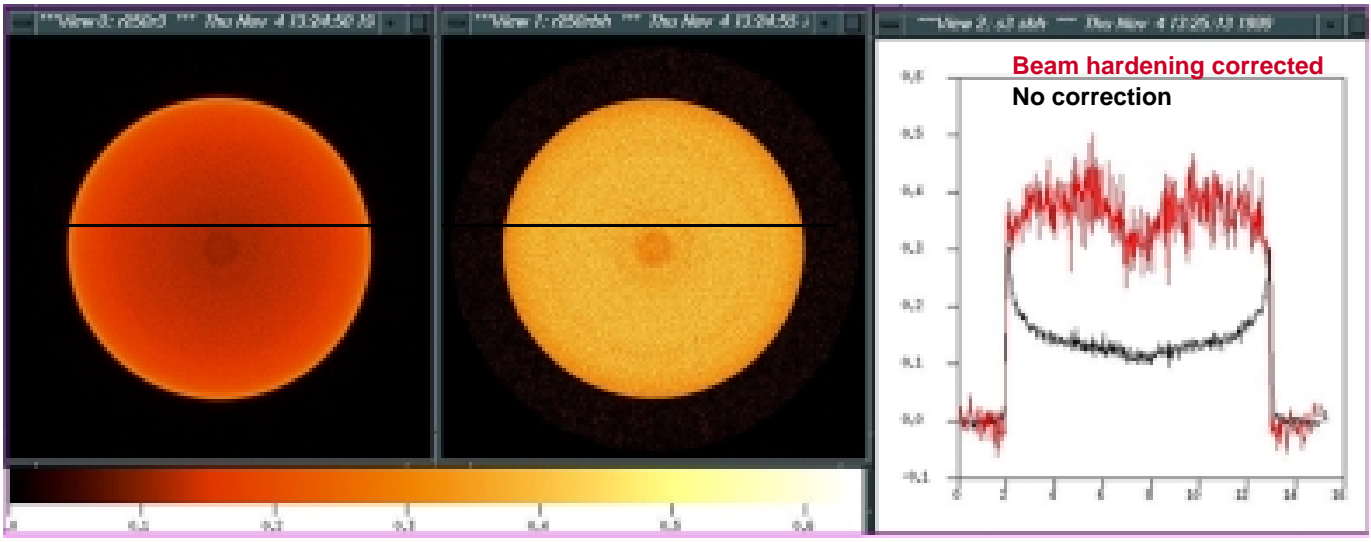

Note: the beam hardening correction increases noise and raises value of CT image. 

S I G N A L / I M A G E
$P$
R O C C E S S S I N G
F O R
N D E

\section{We are studying different quantitative void analysis methods to reduce the CT data}

- We are determining void size, orientation and distribution

in 3-dimensions using codes developed at LLNL

- Using a 'Cluster Labeling' routine (Kinney)

- Create an inverted binary 3-D image (void=1)

- Invert masked image

- Threshold by intensity value determined from histogram

- Apply a median filter of size 3 to thresholded image

- Look for cluster size and connectivity:

- Number/Volume

- Volume fraction

- Size and frequency distribution

- Nearest neighbor distance

\section{Preliminary cluster analysis results - applied to a single 2-D slice}

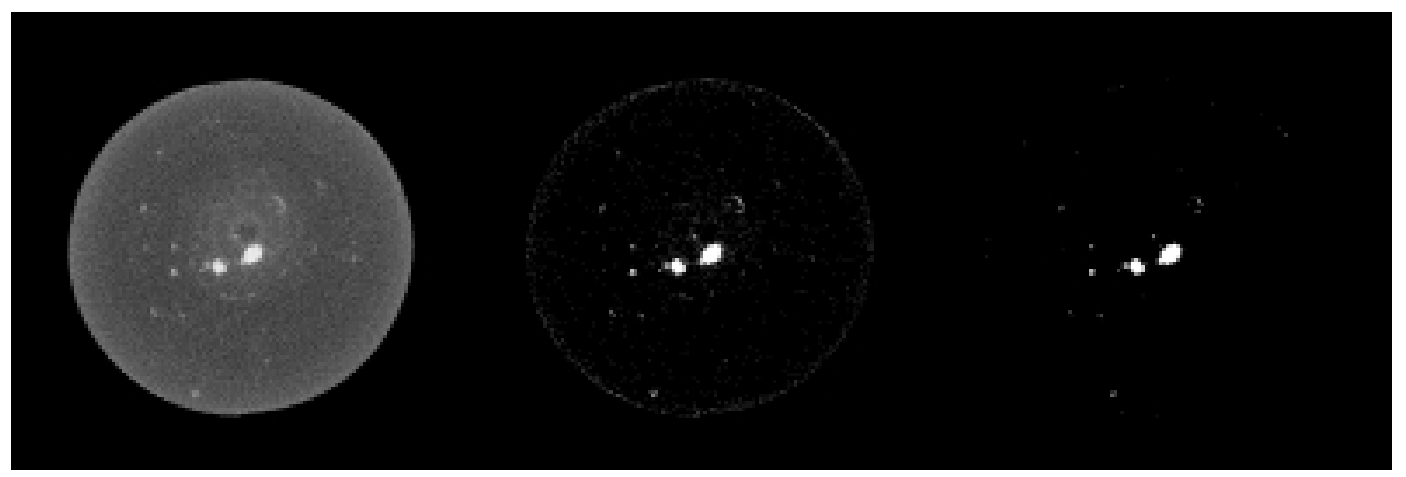

Masked, inverted image (voids appear light)
Thresholded image (determined from histogram)
Median filtered image

$(3 \times 3 \times 3)$

Once the processing parameters are determined the entire CT data set is processed to obtain the 3-D void structure. This data is being incorporated into constitutive material models. 

S I G N A L / I M A G E
P R O C E S S I N G
F O R
N D E

\section{Cluster analysis results}

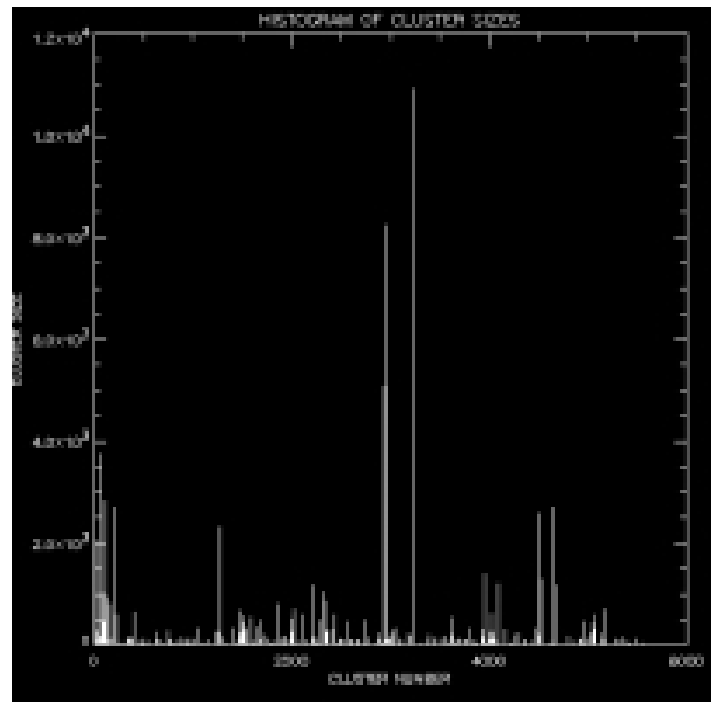

Number and size of voids

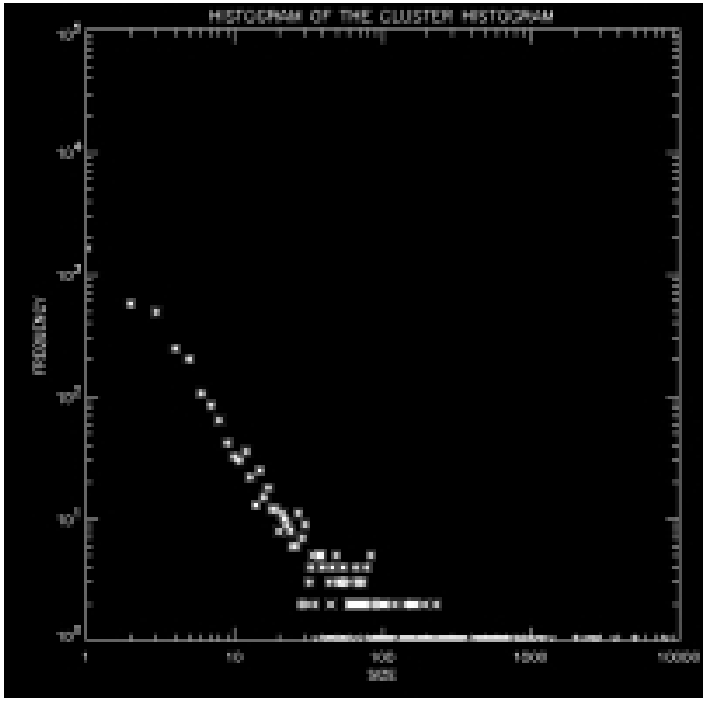

Frequency distribution of voids

\section{Cluster analysis results}

Example of a 3-D surface rendered image of selected voids. This data can be surface meshed and then used with a finite element code for further analysis.
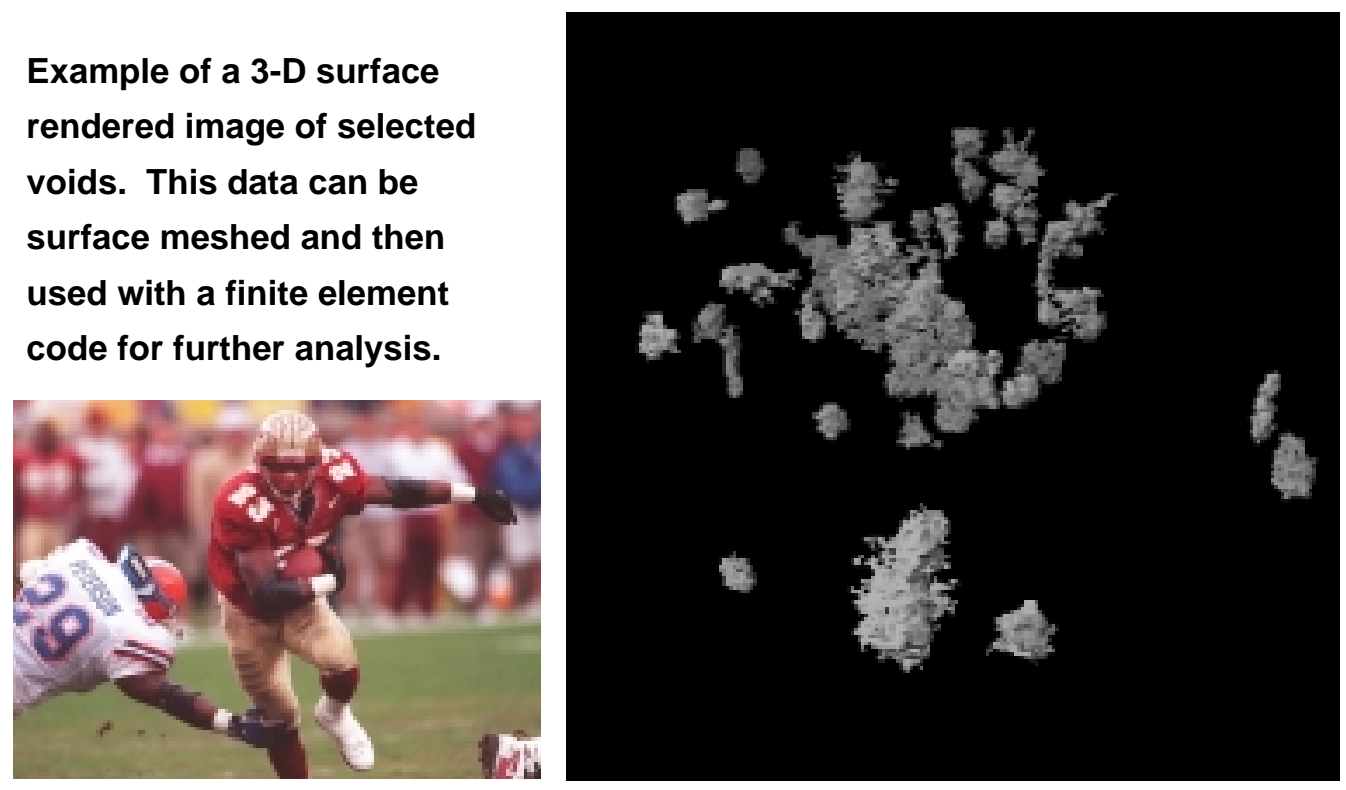

$S$ I G N A L I MAGE
P R O C E S S I N G
F O R
N D E

\section{Summary}

- Simulation code (HADES) is being used to simulate radiographs of tensile specimens to help optimize CT data acquisition, processing and analysis

- CT data have been acquired for all nine samples before loading

- All samples have been loaded to $60 \%$ of their failure loads and rescanned

- Began quantitative void analysis

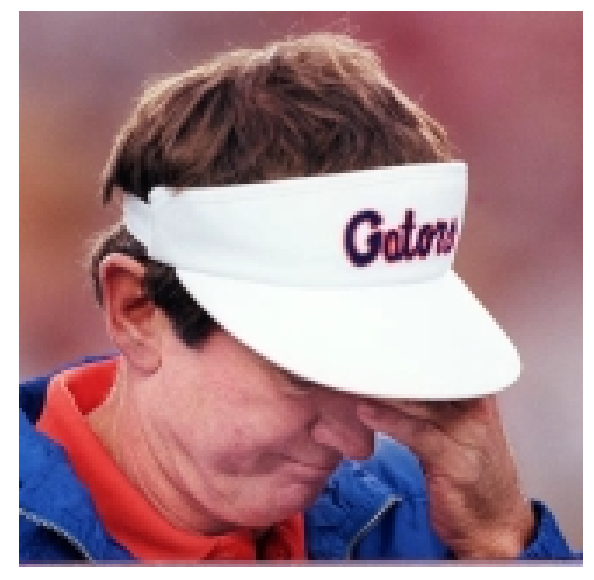
for three $\mathrm{Mg}$ tensile bars (one of each notch geometry)

\section{Future Work}

- Mechanically load samples to remaining percentages of failure; $87 \%, 93 \%$, and $100 \%$, and rescan

- Reconstruct all CT data from experimental and simulated radiographs

- Quantitatively characterize voids e.g., size, orientation and 3-D distribution for all tensile bars, before and after loading

- Segment, decimate and mesh both experimental and simulated data, and perform finite element analysis

- Use empirical void analysis results to change parameters in the constitutive model equations to better fit damage evolution results 
S I G N A L / I M A G E P R O C E S S I N G F O R N D E

Ultrasonic Techniques for Materials Characterization and Process Monitoring: Evaluation of Low Hydrogen Embrittlement Platings on High Tensile Strength Steel

\author{
L. P. Martin and M. Rosen \\ Johns Hopkins University \\ and
}

E.A. Lindgren

Industrial Quality, Inc.

Sacrificial plating is currently applied to high tensile steels used in landing gear applications

- Plating is often based on the Ti-Cd system

- Typical substrate materials are 4330M and 4340M steel

- Significant levels of residual hydrogen may be present after plating

- The residual hydrogen is removed by a subsequent heat treatment (hydrogen bakeout) 
S I G N A L / I M A G E P R O C E S S I N G F O R N D E

The plating process is designed to insure that the electroplated layer remains porous

- The hydrogen removal is critical since these steels can be subject to hydrogen embrittlement cracking

- In order for the bakeout process to be effective, however, it is crucial that the residual hydrogen be able to escape through the plating

- The electroplating procedure is designed to provide porous plating

- Plating characteristics can be affected by bath contamination, current densities, and other process parameters

\section{On line monitoring the porosity level of the LHE} plating is necessary in order to eliminate waste

- Improper plating can lead to hydrogen embrittlement of the component

- Direct testing of individual parts is desirable in order to expedite the manufacturing process and to insure product quality

- Direct testing of individual parts is desirable in order to expedite the manufacturing process and to insure product quality

- Currently used techniques rely on surrogate testing 
S I G N A L / I M A G E P R O C E S S I N G F O R N D E

A surface acoustic wave technique is being evaluated for on line characterization of the LHE plating

- The technique is nondestructive and nonintrusive

- The measurements can be performed rapidly in real-time, and can be used to evaluate every plated component

- Surface waves travel along complex shapes such as curves and corners, so that a wide range of sample geometries can be evaluated

\section{Experimental methodology}

- Measurements of the velocity and amplitude of $5 \mathrm{MHz}$ surface Rayleigh waves have been performed upon LHE plated samples

- A broadband voltage spike was used to excite a commercial $5 \mathrm{MHz}$ longitudinal wave transducer

- This transducer was coupled, using a commercial gel couplant, to an aluminum mode conversion block

- The conversion block was dry coupled to the sample surface with a thin layer of latex 
S I G N A L / I M A G E P R O C E S S I N G F O R N D E

\section{Experimental methodology (cont.)}

- An identical mode conversion block/transducer assembly was used for reception of the signal

- A fixed path length was achieved by mounting the mode conversion blocks on a rail

- Repeatable coupling pressure (against the sample surface) was achieved by placing a fixed mass on top of the mode conversion blocks

- Measurement of the velocity of the Rayleigh wave signal is performed upon an averaged signal of 200 waveforms

\section{Measurement apparatus}
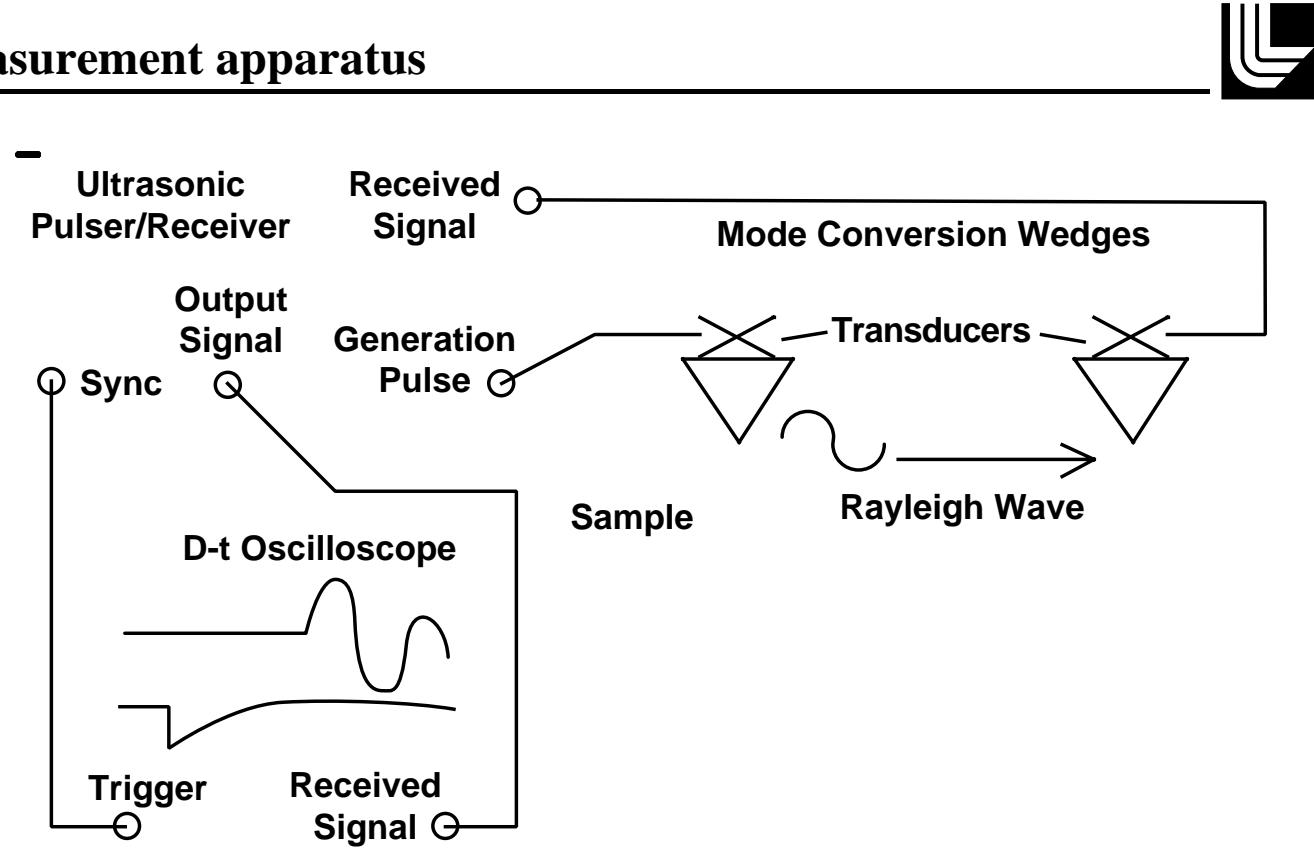
S I G N A L / I M A G E P R O C E S S I N G F O R N D E

Time of flight and peak to peak amplitude were measured from the received Rayleigh wave signals

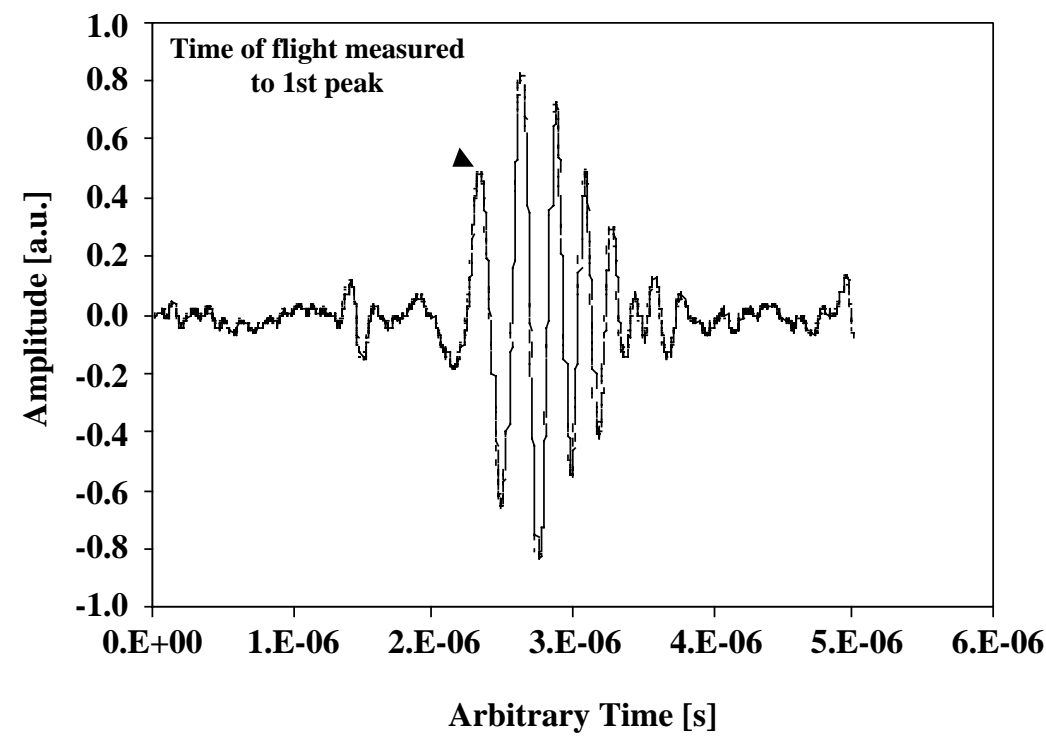

\section{Rayleigh wave velocity and amplitude can} be used to define accept/reject criterion

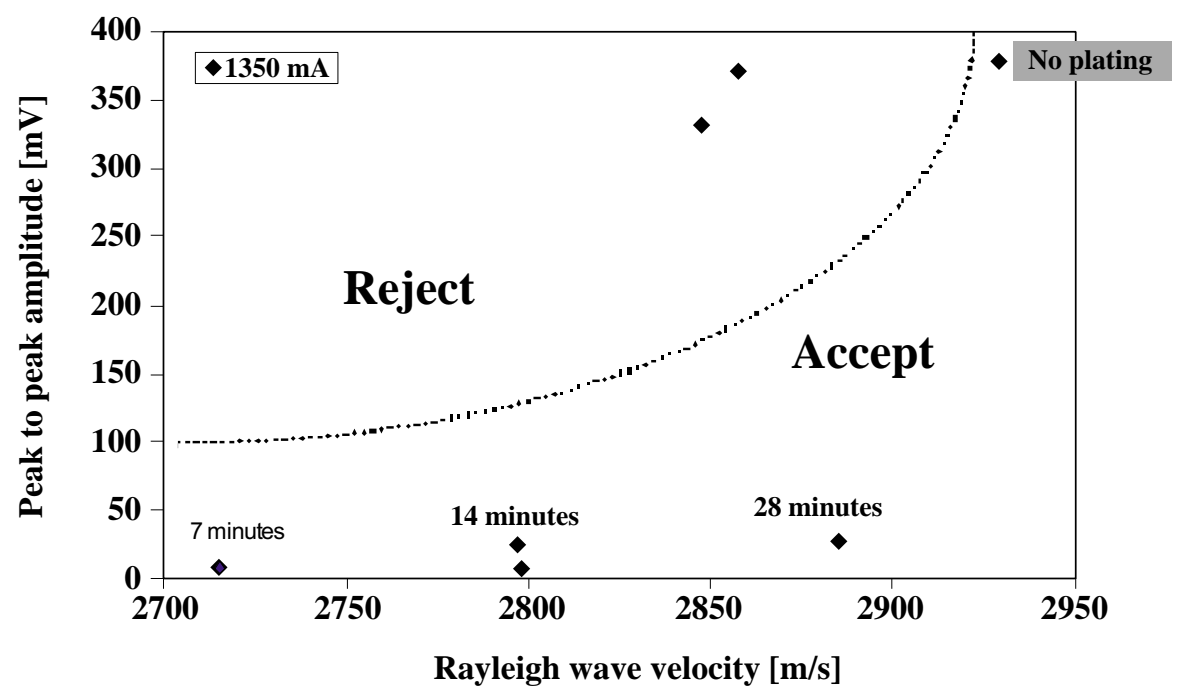


S I G N A L / I M A G E P R O C E S S I N G F O R N D E

The criterion is applicable to samples prepared using a broad range of process parameters

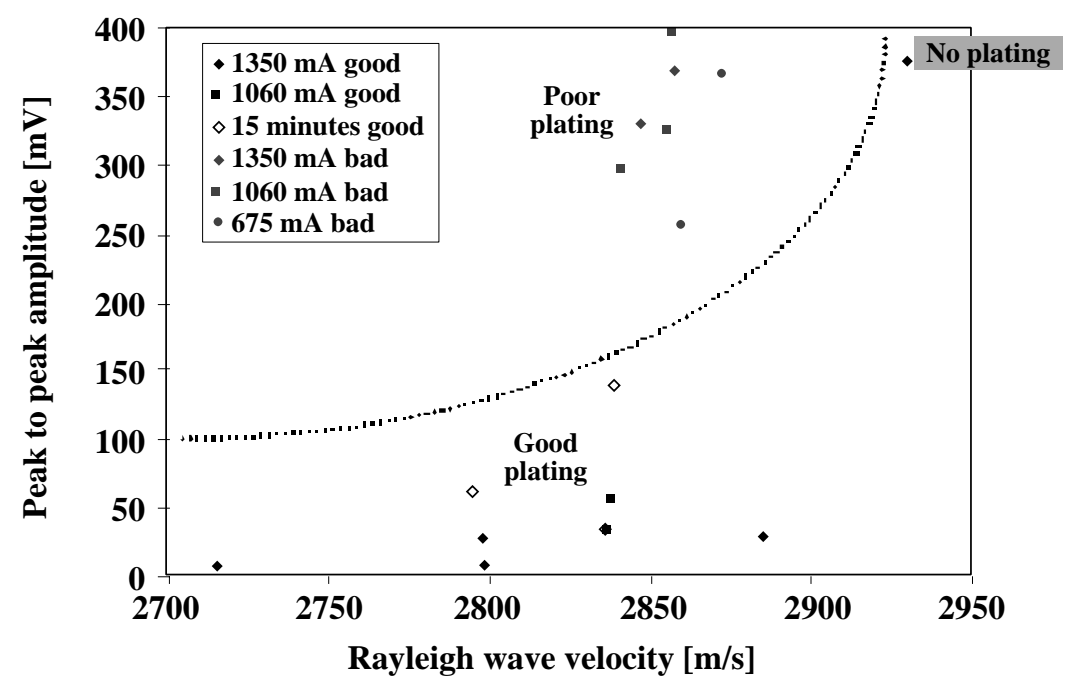

Ipm 4/17/00

NDE-10

\section{Conclusion: The Rayleigh wave technique is a viable} method for on-line characterization of LHE plating

- $\quad$ Rayleigh waves can be generated non-destructively in the materials of interest

- The propagation characteristics of the Rayleigh waves are strongly affected by the plating

- The Rayleigh wave velocity is dominated by the thickness of the plating

- The peak to peak amplitude is dominated by the attenuation (i.e. porosity) in the plating 


\title{
Gamma-ray Imaging with a Segmented Germanium Detector
}

\author{
D. Bekedahl, J.E. Kammeraad, G.J. Schmid, LLNL; \\ J.J. Blair, BECHTEL NEVADA; \\ A. Kuhn, I.Y. Lee, K. Vetter, LBNL
}


